Supporting Information for:

\title{
Expanded Helicenes as Synthons for Chiral Macrocyclic Nanocarbons
}

Gavin R. Kiel ${ }^{\dagger}$, Katherine L. Bay ${ }^{\ddagger}$, Adrian E. Samkian ${ }^{\dagger}$, Nathaniel J. Schuster ${ }^{\S}$, Janice B. Lin ${ }^{\ddagger}$, Rex C. Handford ${ }^{\dagger}$, Colin Nuckolls ${ }^{\S}$, K. N. Houk ${ }^{\ddagger}$, and T. Don Tilley ${ }^{\star \dagger}$

${ }^{\dagger}$ Department of Chemistry, University of California, Berkeley, Berkeley, California, 94720, United States; ${ }^{\circledR}$ Department of Chemistry and Biochemistry, University of California, Los Angeles, Los Angeles, California 90095, United States; $\S$ Department of Chemistry, Columbia University, New York, New York 10027, United States

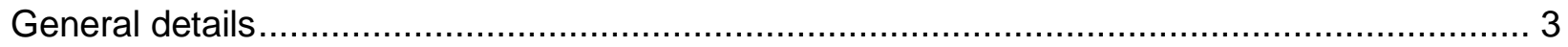

General notes on purification and product isolation ............................................... 3

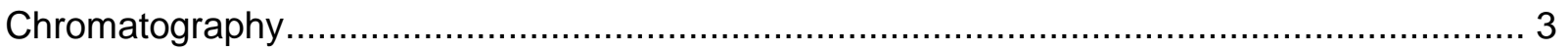

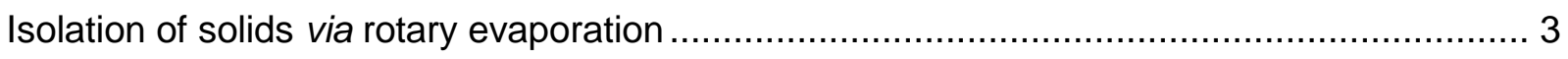

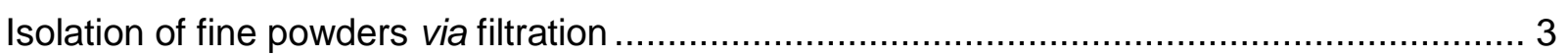

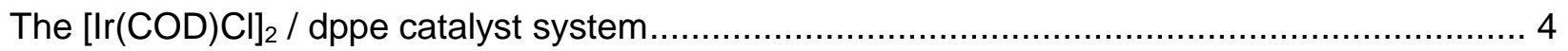

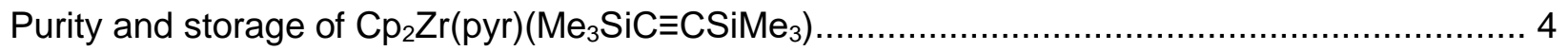

Use of $5 \AA$ molecular sieves (MS) to sequester 4-octyne .............................................. 4

Synthetic procedures and basic characterization data............................................... 5

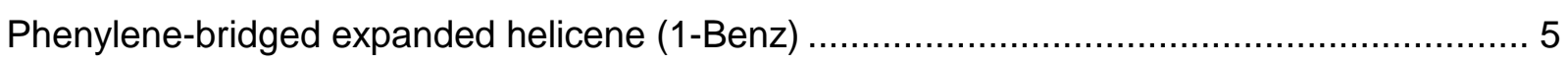

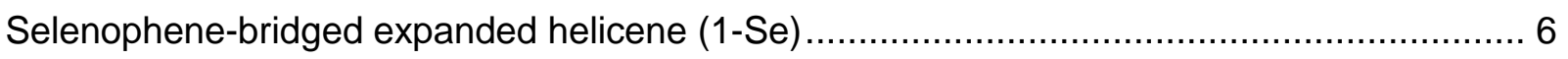

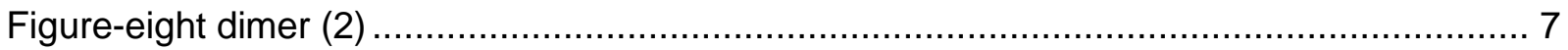

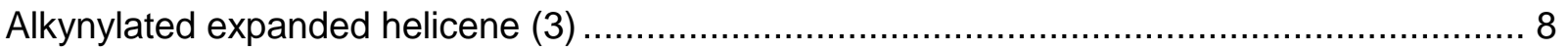

Large scale prep of 3,6-dibromo-2,7-bis(pent-1-ynyl)-9,10-dimethoxyphenanthrene (4) ........ 9

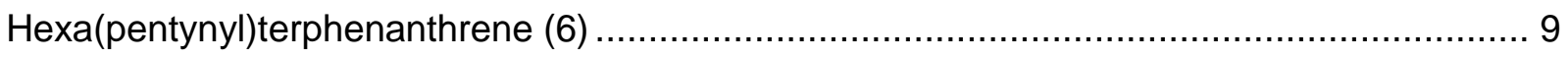

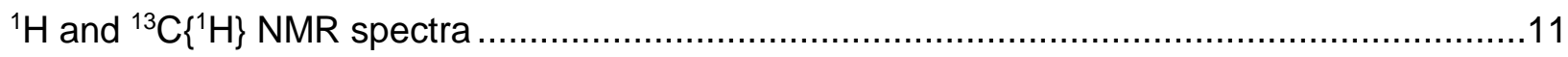

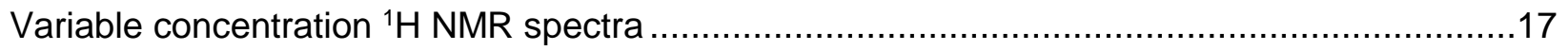

Determination of enantiomerization barriers via dynamic ${ }^{1} \mathrm{H}$ NMR spectroscopy .....................19

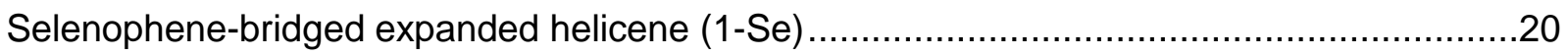

Phenylene-bridged expanded helicene (1-Benz) ........................................................21

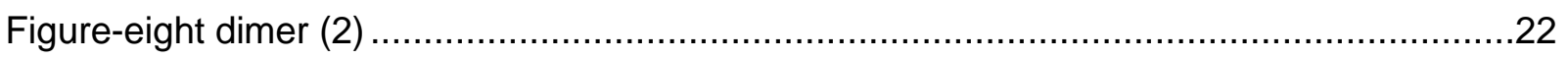

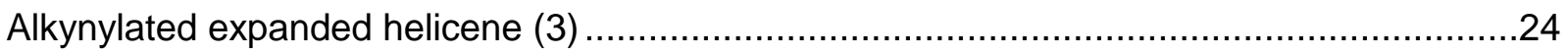

Determination of the enantiomerization barrier of 1-Benz via dynamic circular dichroism (CD)

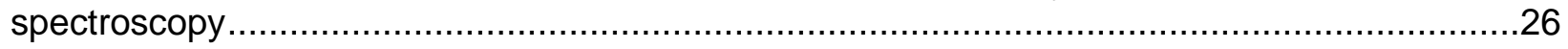

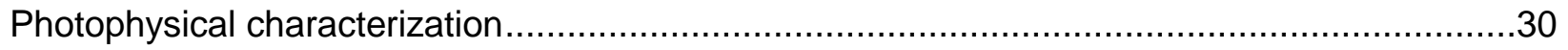

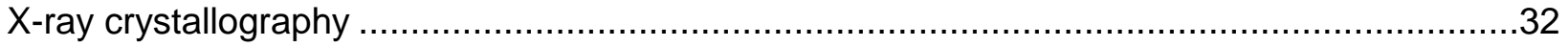

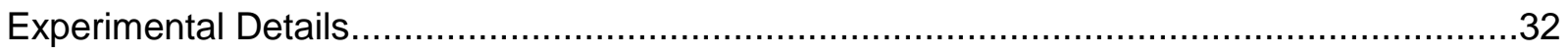


Comparison of X-ray and gas-phase (DFT-calculated) structures................................33

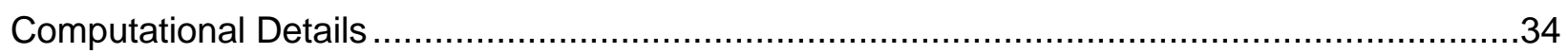

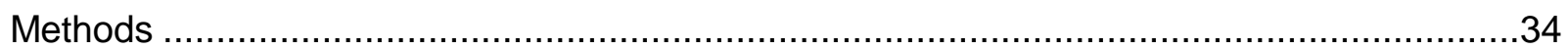

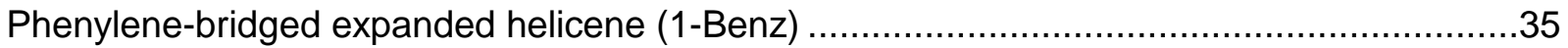

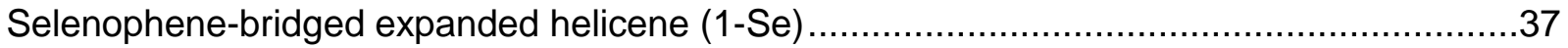

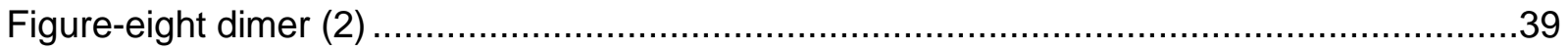

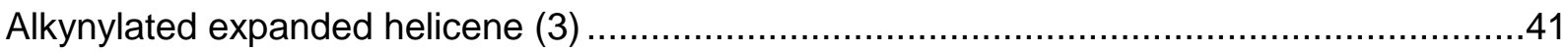

Summary of energies and vibrational frequencies ......................................................43

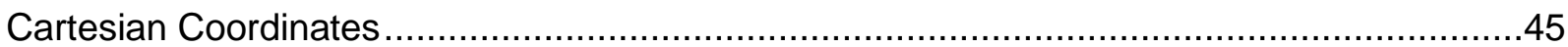

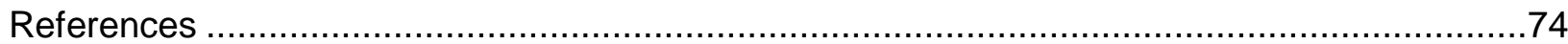




\section{General details}

Unless otherwise stated, all manipulations of organometallic compounds were carried out in dry solvents under an atmosphere of nitrogen, using either standard Schlenk techniques or a glovebox. Toluene and tetrahydrofuran (THF) were dried using a JC Meyers Phoenix SDS solvent purification system. Benzene was dried using a Vacuum Atmosphere solvent purification system. Benzene- $d_{6}$ and DMPU were freed from oxygen using three free-pump-thaw cycles and then dried for at least $48 \mathrm{~h}$ over $3 \AA$ molecular sieves (5\% by mass). All reaction solvents were stored over $3 \AA$ molecular sieves. $\mathrm{Cp}_{2} \mathrm{Zr}(\mathrm{pyr})\left(\mathrm{Me}_{3} \mathrm{SiC} \equiv \mathrm{CSiMe}_{3}\right),{ }^{1} \mathrm{SeCl}_{2}$ (bipy), ${ }^{2}[\operatorname{Ir}(\mathrm{COD}) \mathrm{Cl}]_{2},{ }^{3}$ 1, 4-dimethoxy2-butyne, ${ }^{4} \quad \mathrm{EtC} \equiv \mathrm{Mo}\left(\mathrm{OC}\left(\mathrm{CH}_{3}\right)\left(\mathrm{CF}_{3}\right)_{2}\right)_{3}(\mathrm{DME}),{ }^{5} \operatorname{Ir}(\mathrm{COD})(\mathrm{dppe}) \mathrm{Cl},{ }^{6}$ 3,6-dibromo-2,7-diiodo-9,10dimethoxyphenanthrene, ${ }^{7} \quad$ 3-bromo-9,10-dimethoxy-2,7-di(pent-1-yn-1-yl)phenanthrene $\quad$ (5), ${ }^{8}$ i $\mathrm{PrMgCl} \cdot \mathrm{LiCl}^{9}$ and $\mathrm{PdCl}_{2}(\mathrm{IPr})\left(3\right.$-chloropyridine) ${ }^{10}$ were prepared by literature procedures or slight modifications thereof. 'PrMgCl- $\mathrm{LiCl}$ was titrated by ${ }^{1} \mathrm{H}$ NMR spectroscopy immediately prior to use. ${ }^{11}$ All other reagents and solvents were purchased from commercial suppliers and used as received. "Room temperature", "RT", or "ambient temperature" refers to $\sim 22{ }^{\circ} \mathrm{C}$. Reaction temperatures represent the oil bath temperature (with a fully submersed, stirred solution) unless otherwise stated. MALDI-TOF mass spectrometry was performed at the Mass Spectrometry Facility at UC Riverside. a-Cyano-4-hydroxycinnamic acid was used as the matrix. Unless otherwise noted, NMR spectra were acquired at ambient temperature $\left(\sim 22^{\circ} \mathrm{C}\right)$ using Bruker AV600, AV-500, DRX-500, AV-400, and AV-300 spectrometers. Chemical shifts $(\delta)$ are given in ppm and referenced to residual solvent peaks for ${ }^{1} \mathrm{H}$ NMR spectra $(\delta=7.26 \mathrm{ppm}$ for chloroform- $d, \delta=$ 7.16 for benzene- $d_{6}, \delta=5.32$ for dichloromethane- $d_{2}$, and $\delta=2.09$ for toluene- $d_{8}$ ) and for ${ }^{13} \mathrm{C}\left\{{ }^{1} \mathrm{H}\right\}$ NMR spectra $(\delta=77.16 \mathrm{ppm}$ for chloroform- $d)$. ${ }^{1} \mathrm{H}$ NMR yields were determined using $1,3,5-$ trimethoxybenzene as internal standard. For greatest accuracy, yields were typically measured by acquisition of ${ }^{1} \mathrm{H}$ NMR spectra both before and after the reaction of interest, followed by a comparison of the amounts of starting material and desired product relative to the amount of added internal standard.

\section{General notes on purification and product isolation}

\section{Chromatography}

Column chromatography was carried out using Fischer Chemical 40-63 $\mu \mathrm{m}, 230-400$ mesh silica gel. Preparatory thin layer chromatography (prep TLC) was carried out using Analtech Silica Gel GF UNIPLATES $(1000 \mu \mathrm{m}, 20 \times 20 \mathrm{~cm})$. For prep TLC, in a typical procedure, 35-50 mg of material was loaded onto one side of the plate and the solvent front was allowed to elute halfway up (i.e. $70-100 \mathrm{mg}$ was separated per plate).

\section{Isolation of solids via rotary evaporation}

For most of the new compounds, the concentration of their solutions in "good" solvents (e.g. $\mathrm{CH}_{2} \mathrm{Cl}_{2}$ ) typically furnished a glassy, yellow/orange residue. For isolation of these compounds in powder form, co-evaporation with a poor solvent (usually hexanes) was necessary. Typically, the solution (e.g. the extracted solution from prep TLC) was concentrated via rotary evaporation in a round-bottom flask, then the residue was transferred to a $20 \mathrm{~mL}$ vial with the minimal amount of $\mathrm{CH}_{2} \mathrm{Cl}_{2}$. The $\mathrm{CH}_{2} \mathrm{Cl}_{2}$ solution was then co-evaporated with hexanes via rotary evaporation, and residual solvents were removed under high vacuum to give the desired powder. This can be repeated with a larger hexanes $/ \mathrm{CH}_{2} \mathrm{Cl}_{2}$ ratio if a powder is not obtained.

\section{Isolation of fine powders via filtration}

Several procedures below involve the precipitation of the desired product with an anti-solvent (e.g. $\mathrm{MeOH}$ ). This often produced a very fine solid, which made filtration difficult. In these cases, 
filtration was greatly aided by celite, which was then extracted with $\mathrm{CH}_{2} \mathrm{Cl}_{2}$ for quantitative recovery of product. See above for important notes regarding rotary evaporation of $\mathrm{CH}_{2} \mathrm{Cl}_{2}$ solutions.

\section{The $[\operatorname{Ir}(\mathrm{COD}) \mathrm{Cl}]_{2} /$ dppe catalyst system}

As discussed in our previous report, ${ }^{8}$ the reaction of 1.0 equiv of $[\operatorname{Ir}(C O D) C l]_{2}$ with 2.0 equiv of dppe (in toluene or benzene) gives the [2+2+2] pre-catalyst $\operatorname{Ir}(\mathrm{COD})(\mathrm{dppe}) \mathrm{Cl}$. If dppe is present in excess, an orange/red precipitate (likely $\operatorname{Ir}(\mathrm{dppe})_{2} \mathrm{Cl}^{12,13}$ ) is observed. The most reproducible way to achieve the desired catalyst loading is with isolated $\operatorname{Ir}(\mathrm{COD})(\mathrm{dppe}) \mathrm{Cl}$ (prepared in a simple one-step procedure ${ }^{6}$ ). For some procedures below, $\operatorname{Ir}(\mathrm{COD})(\mathrm{dppe}) \mathrm{Cl}$ was pre-formed in situ by addition of a solution of dppe in toluene to a solution of $[\operatorname{Ir}(\mathrm{COD}) \mathrm{Cl}]_{2}$ in toluene.

\section{Purity and storage of $\mathrm{Cp}_{2} \mathrm{Zr}(\mathrm{pyr})\left(\mathrm{Me}_{3} \mathrm{SiC} \equiv \mathrm{CSiMe}_{3}\right)$}

This compound undergoes some decomposition on storage at ambient temperature; thus, it was stored at $-35^{\circ} \mathrm{C}$ (in an $\mathrm{N}_{2}$-filled glovebox). In the procedures below, the reagent's purity was typically $90-95 \%$, as determined by ${ }^{1} \mathrm{H}$ NMR spectroscopy in benzene- $d_{6}$ (using hexamethylbenzene as internal standard). The masses reported below reflect the quantity of active species and the impurities do not noticeably affect results.

\section{Use of $5 \AA$ molecular sieves (MS) to sequester 4-octyne}

We observed that the source of the molecular sieves is of critical importance for the alkyne metathesis reactions performed herein. All successful reactions used pre-powdered $5 \AA$ MS that were purchased from Sigma Aldrich and activated under high vacuum at $\sim 300{ }^{\circ} \mathrm{C}^{*}$ (until condensation of water ceased, then for another $24 \mathrm{~h}$ ). The activated $5 \AA$ MS were then stored in a solvent-free, nitrogen-filled glovebox until use. At the outset of our investigations, beaded $5 \AA$ MS (3-5 mm, from Alfa Aesar) were activated as described above, then thoroughly ground with a mortar and pestle in a solvent-free, nitrogen-filled glovebox. Use of these molecular sieves was not effective for the sequestration of 4-octyne.

${ }^{*}$ Temperature of the sand bath. 


\section{Synthetic procedures and basic characterization data}

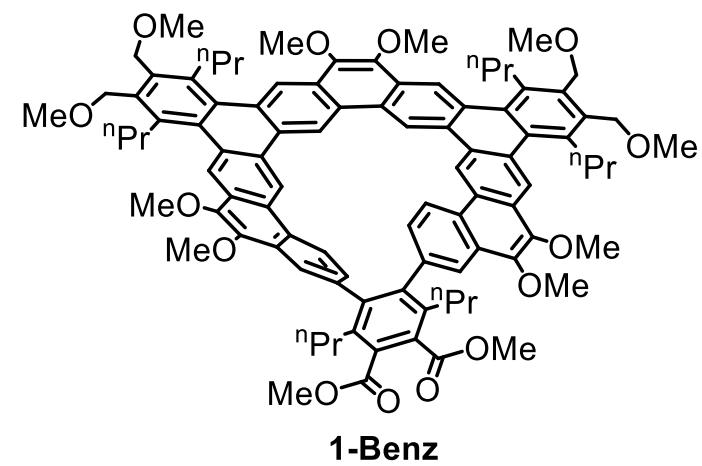

Phenylene-bridged expanded helicene (1-Benz)

Procedure \#1 (one-pot, from hexayne 6): To a solution of [Ir(COD)Cl] 2 (1.2 mg, $0.0018 \mathrm{mmol}, 0.02$ equiv) in benzene $(0.5 \mathrm{~mL}$ ) was added (dropwise over $\sim 1 \mathrm{~min})$ a solution of dppe $(1.4 \mathrm{mg}, 0.0036$ mmol, 0.04 equiv) in benzene $(0.5 \mathrm{~mL})$. This mixture was immediately added to a flask containing a solution of hexayne 6 (101 mg, $0.0909 \mathrm{mmol}, 1.0$ equiv), 1,4-dimethoxy-2-butyne (31 mg, 0.273 mmol, 3.0 equiv), and 1,3,5-trimethoxybenzene (14.6 mg, internal standard) in benzene $(0.8 \mathrm{~mL})$. The flask was sealed with a Teflon stopper and the reaction mixture was heated at $80^{\circ} \mathrm{C}$ for $2 \mathrm{~h}$. The mixture was allowed to cool to $\mathrm{RT}^{*}$ and diluted with benzene $(6 \mathrm{~mL}$, to bring total concentration to $0.012 \mathrm{M}$ relative to starting 6$)$. To the stirred mixture was added, dropwise over 5-10 min, a solution of $\mathrm{Cp}_{2} \mathrm{Zr}\left(\mathrm{Me}_{3} \mathrm{SiC} \equiv \mathrm{CSiMe}_{3}\right)$ pyr (64 mg, $0.136 \mathrm{mmol}, 1.5$ equiv) in benzene $(1.0 \mathrm{~mL}) .{ }^{* *}$ The mixture was stirred for $10 \mathrm{~min}$ at $\mathrm{RT},{ }^{* * *}$ then DMAD (39 mg, $0.27 \mathrm{mmol}, 3.0$ equiv), DMPU (70 mg, $0.55 \mathrm{mmol}, 6.0$ equiv), and copper(I) chloride (27 mg, $0.27 \mathrm{mmol}, 3.0$ equiv) were added in that order. The mixture was stirred for $1 \mathrm{~h}$ at RT and directly subjected (in air) to column chromatography $\left(0-10 \%\right.$ EtOAc in $\left.\mathrm{CH}_{2} \mathrm{Cl}_{2}\right)$ to give a light-yellow solid that was a mixture of 1Benz and DMAD. To remove DMAD, the solid was dissolved in $\mathrm{CH}_{2} \mathrm{Cl}_{2}$ (3-4 mL), filtered, then the filtrate was concentrated to $\sim 2 \mathrm{~mL}$. To this solution was added $\mathrm{EtOH}(6 \mathrm{~mL})$, then the solution was concentrated to half of its volume to give a finely dispersed precipitate. The precipitate was collected by filtration $^{\star * * *}$ and washed with $\mathrm{MeOH}(2 \times 3 \mathrm{~mL})$ to afford 1-Benz (68 $\left.\mathrm{mg}, 51 \%\right)$ as a light-yellow solid.

Notes for Procedure \#1: *Analysis of an aliquot by ${ }^{1} \mathrm{H}$ NMR spectroscopy revealed formation of helicene 3 in $85 \%$ yield. ${ }^{*}$ Order of addition and concentration play a critical role in the generation of the intermediate macrocyclic zirconacyclopentadiene $\mathbf{1 - Z r}$ in high yield. ${ }^{* * *}$ Analysis of an aliquot by ${ }^{1} \mathrm{H}$ NMR spectroscopy revealed formation of $1-Z r$ in $65 \%$ yield (from hexayne 6 ). ${ }^{* \star \star *}$ The solid was very fine and filtration was greatly aided by celite. After filtration, the celite was extracted with $\mathrm{CH}_{2} \mathrm{Cl}_{2}$ for recovery of product (see the section titled "general notes on purification").

Procedure \#2 (from isolated helicene 3): This procedure was identical to Procedure 1 above (starting with formation of $1-\mathrm{Zr}$ ), except 1.2 equiv of $\mathrm{Cp}_{2} \mathrm{Zr}\left(\mathrm{Me}_{3} \mathrm{SiC} \equiv \mathrm{CSiMe}_{3}\right)$ pyr was used. The isolated yield of 1-Benz was $65 \%$ (from 3 ).

Characterization: ${ }^{1} \mathrm{H}$ NMR (chloroform- $\left.d, 500 \mathrm{MHz},[\mathrm{c}]=2.6 \mathrm{mM}\right): \delta=9.83(\mathrm{~s}, 2 \mathrm{H}), 9.67(\mathrm{~s}, 2 \mathrm{H})$, $9.23(\mathrm{~s}, 2 \mathrm{H}), 8.84(\mathrm{~s}, 2 \mathrm{H}), 8.42(\mathrm{~d}, J=8.5 \mathrm{~Hz}, 2 \mathrm{H}), 8.12(\mathrm{~d}, J=1.9 \mathrm{~Hz}, 2 \mathrm{H}), 6.56(\mathrm{dd}, J=8.3,1.8$ $\mathrm{Hz}, 2 \mathrm{H}), 4.87(\mathrm{~d}, J=10.4 \mathrm{~Hz}, 2 \mathrm{H}), 4.82(\mathrm{~d}, J=10.2 \mathrm{~Hz}, 2 \mathrm{H}), 4.81(\mathrm{~d}, J=10.2 \mathrm{~Hz}, 2 \mathrm{H}), 4.77$ (d, J $=10.4 \mathrm{~Hz}, 2 \mathrm{H}), 4.20(\mathrm{~s}, 6 \mathrm{H}), 4.18(\mathrm{~s}, 6 \mathrm{H}), 4.17(\mathrm{~s}, 6 \mathrm{H}), 4.00(\mathrm{~s}, 6 \mathrm{H}), 3.61(\mathrm{~s}, 6 \mathrm{H}), 3.60(\mathrm{~s}, 6 \mathrm{H})$, $3.60-3.35(\mathrm{~m}, 8 \mathrm{H}), 3.21$ (ddd, $J=14.8,10.3,5.9 \mathrm{~Hz}, 2 \mathrm{H}), 2.86$ (ddd, $J=14.4,10.2,5.0 \mathrm{~Hz}, 2 \mathrm{H}$ ), $2.26-2.09(\mathrm{~m}, 4 \mathrm{H}), 2.09-1.97(\mathrm{~m}, 2 \mathrm{H}), 1.97-1.82(\mathrm{~m}, 2 \mathrm{H}), 1.64-1.52(\mathrm{~m}, 2 \mathrm{H}), 1.44-1.34$ $(\mathrm{m}, 2 \mathrm{H}), 1.28(\mathrm{t}, J=7.3 \mathrm{~Hz}, 6 \mathrm{H}), 1.05(\mathrm{t}, J=7.2 \mathrm{~Hz}, 6 \mathrm{H}), 0.72(\mathrm{t}, J=7.3 \mathrm{~Hz}, 6 \mathrm{H}) ;{ }^{13} \mathrm{C}\left\{{ }^{1} \mathrm{H}\right\} \mathrm{NMR}$ (chloroform- $d, 101 \mathrm{MHz}$ ): $\delta=169.87,144.64,144.48,144.22,143.78,137.35,137.18,137.08$, 
$136.65,136.19,134.69,133.14,132.64,130.74,130.65,130.39,130.23,129.13,129.02,128.59$, 127.85, 127.69, 127.56, 126.74, 126.21, 122.15, 121.91, 120.45, 120.37, 120.19, 118.58, 69.05, 68.95, 61.18, 61.03, 60.78, 58.96, 58.79, 52.71, 35.28, 35.02, 33.68, 25.94, 25.03, 25.01, 14.50, 14.49, 14.31; HRMS-MALDI-TOF (m/z): [M] ${ }^{+}$calcd. for $\mathrm{C}_{96} \mathrm{H}_{100} \mathrm{O}_{14}, 1476.7113$; found, 1476.6602.

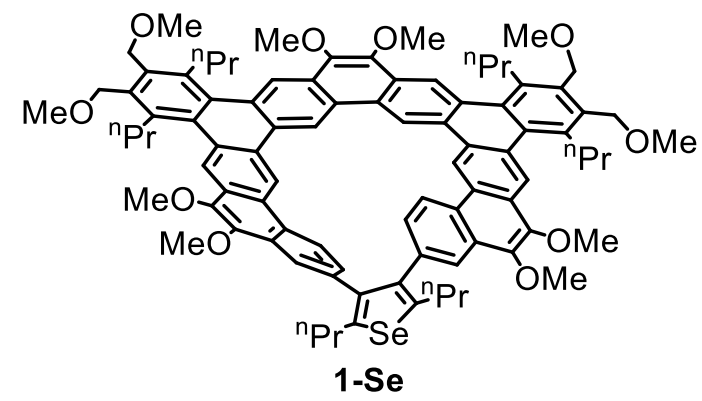

\section{Selenophene-bridged expanded helicene (1-Se)}

Procedure: To a stirred solution of helicene 3 (100 mg, $0.0749 \mathrm{mmol}, 1.0$ equiv) in toluene $(6 \mathrm{~mL})$ was added, dropwise over $5-10 \mathrm{~min}$, a solution of $\mathrm{Cp}_{2} \mathrm{Zr}\left(\mathrm{Me}_{3} \mathrm{SiC} \equiv \mathrm{CSiMe}\right.$ ) pyr (42 mg, 0.090 mmol, 1.2 equiv) in toluene $(1.0 \mathrm{~mL})$. ${ }^{*}$ The mixture was stirred for $5 \mathrm{~min}$ at $\mathrm{RT}$, then $\mathrm{SeCl}_{2}($ bipy) (46 mg, $0.15 \mathrm{mmol}, 2.0$ equiv) was added and the resulting suspension was stirred for a further 5 min. In air, the suspension was filtered through celite and the filter cake was rinsed with toluene $(2 \times 3 \mathrm{~mL})$. Addition of $\mathrm{MeOH}(75 \mathrm{~mL})$ to the filtrate resulted in the formation of a fine yellow/orange precipitate, which was collected via filtration** and washed with $\mathrm{MeOH}(2 \times 5 \mathrm{~mL})$. Subjection of the crude product to preparatory thin layer chromatography afforded 1-Se (75 $\mathrm{mg}, 71 \%$ ) as a paleyellow solid.

Notes: * Order of addition and concentration play a critical role in the generation of the intermediate macrocyclic zirconacyclopentadiene 1-Zr in high yield. Using this procedure, 1-Zr was observed in $86 \%$ yield (by ${ }^{1} \mathrm{H}$ NMR spectroscopy). ${ }^{* *}$ The solid was very fine and filtration was greatly aided by celite. After filtration, the celite was extracted with $\mathrm{CH}_{2} \mathrm{Cl}_{2}$ for recovery of product (see the section titled "general notes on purification").

Characterization: ${ }^{1} \mathrm{H}$ NMR (chloroform- $d, 400 \mathrm{MHz},[\mathrm{c}]=6.2 \mathrm{mM}$ ): $\delta=9.91(\mathrm{~s}, 2 \mathrm{H}), 9.75(\mathrm{~s}, 2 \mathrm{H}$ ), $9.21(\mathrm{~s}, 2 \mathrm{H}), 8.84(\mathrm{~s}, 2 \mathrm{H}), 8.53(\mathrm{~d}, J=8.5 \mathrm{~Hz}, 2 \mathrm{H}), 8.34(\mathrm{~d}, J=1.8 \mathrm{~Hz}, 2 \mathrm{H}), 6.64$ (dd, $J=8.4,1.8$ $\mathrm{Hz}, 2 \mathrm{H}), 4.88(\mathrm{~d}, J=10.3 \mathrm{~Hz}, 2 \mathrm{H}), 4.83(\mathrm{~d}, J=10.1 \mathrm{~Hz}, 2 \mathrm{H}), 4.81(\mathrm{~d}, J=10.7 \mathrm{~Hz}, 2 \mathrm{H}), 4.78(\mathrm{~d}, J$ $=10.5 \mathrm{~Hz}, 2 \mathrm{H}), 4.21(\mathrm{~s}, 6 \mathrm{H}), 4.21(\mathrm{~s}, 6 \mathrm{H}), 4.17(\mathrm{~s}, 6 \mathrm{H}), 3.61(\mathrm{~s}, 6 \mathrm{H}), 3.60(\mathrm{~s}, 6 \mathrm{H}), 3.67-3.40(\mathrm{~m}$, $8 \mathrm{H}), 3.41-3.33(\mathrm{~m}, 2 \mathrm{H}), 3.26-3.16(\mathrm{~m}, 2 \mathrm{H}), 2.29-1.79(\mathrm{~m}, 12 \mathrm{H}), 1.25(\mathrm{t}, J=7.2 \mathrm{~Hz}, 6 \mathrm{H}), 1.13$ (t, $J=7.3 \mathrm{~Hz}, 6 \mathrm{H}), 1.02$ (t, $J=7.2 \mathrm{~Hz}, 6 \mathrm{H}$ ); ${ }^{13} \mathrm{C}\left\{{ }^{1} \mathrm{H}\right\}$ NMR (chloroform- $d, 151 \mathrm{MHz}$ ): $\delta=146.19$, 144.64, 144.61, 144.16, 140.67, 137.31, 137.29, 136.52, 136.17, 136.12, 134.80, 133.41, 130.95, 130.60, 130.44, 130.14, 130.06, 129.31, 128.60, 127.83, 127.79, 127.60, 127.10, 125.00, 122.45, $121.78,120.45,119.79,118.84,69.29,69.06,61.26,61.13,61.02,58.95,58.85,35.36,35.04$, 34.12, 27.21, 26.10, 25.17, 14.57, 14.55, 14.25; HRMS-MALDI-TOF (m/z): [M] ${ }^{+}$calcd. for $\mathrm{C}_{90} \mathrm{H}_{94} \mathrm{O}_{10} \mathrm{Se}, 1414.6012$; found, 1414.5823. 


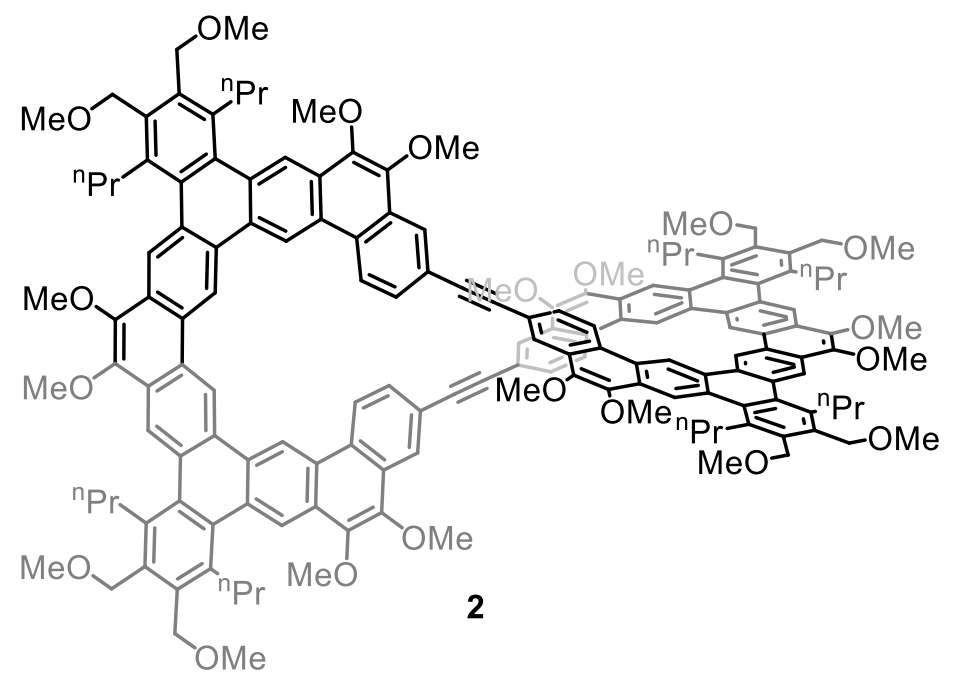

Figure-eight dimer (2)

Procedure \#1 (from helicene 3, no $5 \AA$ MS): A J-Young NMR tube was charged with 3 (50 mg, $0.0374 \mathrm{mmol}, 1.0$ equiv), 1,3,5-trimethoxybenzene ( $\sim 5 \mathrm{mg}$, internal standard), $\mathrm{EtC} \equiv \mathrm{Mo}\left(\mathrm{OC}\left(\mathrm{CH}_{3}\right)\left(\mathrm{CF}_{3}\right)_{2}\right)_{3}(\mathrm{DME})\left(2.9 \mathrm{mg}, 0.0037 \mathrm{mmol}, 0.10\right.$ equiv), and benzene- $d_{6}(0.75 \mathrm{~mL})$. The tube was sealed, and the mixture was heated at $80^{\circ} \mathrm{C}$ for $16 \mathrm{~h}$. Analyses of the mixture by ${ }^{1} \mathrm{H}$ NMR spectroscopy after $3 \mathrm{~h}$ and $16 \mathrm{~h}$ (see Figure S7d) both revealed $95 \%$ consumption of starting 3 and $93 \%$ yield of 2 (i.e. conversion halted after $3 \mathrm{~h}$ ). The mixture was directly subjected to preparatory thin layer chromatography (4\% $\mathrm{EtOAc}$ in $\left.\mathrm{CH}_{2} \mathrm{Cl}_{2}\right)$, which afforded $2(41 \mathrm{mg}, 89 \%)$ as a yellow solid.

Procedure \#2 (from helicene 3, with $5 \AA$ MS): A $10 \mathrm{~mL}$ Teflon-stoppered flask with magnetic stirbar was charged with 3 (100 mg, $0.0749 \mathrm{mmol}, 1.0$ equiv), $\mathrm{EtC} \equiv \mathrm{Mo}\left(\mathrm{OC}\left(\mathrm{CH}_{3}\right)\left(\mathrm{CF}_{3}\right)_{2}\right)_{3}(\mathrm{DME})(5.8 \mathrm{mg}$, $0.0075 \mathrm{mmol}, 0.10$ equiv), powdered $5 \AA$ molecular sieves* $(150 \mathrm{mg})$, and benzene- $d_{6}(3.0 \mathrm{~mL})$. The flask was sealed, then the stirred mixture was heated at $80^{\circ} \mathrm{C}$ for $16 \mathrm{~h}$, cooled to RT, and an aliquot was filtered into an NMR tube. Analysis by ${ }^{1} \mathrm{H}$ NMR spectroscopy revealed complete consumption of $\mathbf{3}$ and the absence of byproduct 4-octyne (see Figure S7e). The mixture was quantitatively transferred to a $50 \mathrm{~mL}$ round-bottom flask with the aid of toluene $(2 \times 2 \mathrm{~mL})$, and silica gel $(1 \mathrm{~g})$ was added. Volatile materials were removed by rotary evaporation and the resulting powder was layered onto a plug of silica gel $(5 \mathrm{~g})$ that had been equilibrated with $10 \%$ EtOAc in $\mathrm{CH}_{2} \mathrm{Cl}_{2}$. The plug was flushed with the same solvent mixture until the yellow color was very faint ( $50 \mathrm{~mL})$. Removal of solvents ${ }^{* *}$ from the eluent via rotary evaporation afforded $2(87 \mathrm{mg}, 95 \%)$ as a yellow solid.

Notes for Procedure \#2: *See general section (above) for important details on the source of the molecular sieves. ${ }^{* *}$ See the section titled "general notes on purification" for important details.

Procedure \#3 (one pot, gram-scale, from hexayne 6): A $100 \mathrm{~mL}$ Teflon-stoppered flask was loaded with hexayne 6 (1.40 g, $1.26 \mathrm{mmol}, 1.0$ equiv), 1,4-dimethoxy-2-butyne (0.575 g, 5.04 $\mathrm{mmol}, 4.0$ equiv), and toluene $(15 \mathrm{~mL})$, and to this flask was added a solution of $\operatorname{lr}(\mathrm{COD})(\mathrm{dppe}) \mathrm{Cl}$ $(0.037 \mathrm{~g}, 0.050 \mathrm{mmol}, 0.04$ equiv) in toluene $(10 \mathrm{~mL})$. The flask was sealed with a Teflon stopper and the stirred reaction mixture was heated at $80^{\circ} \mathrm{C}$ for $2 \mathrm{~h}$. The mixture was allowed to cool to RT, then powdered $5 \AA$ molecular sieves ${ }^{*}(3.8 \mathrm{~g})$ and a solution of $\mathrm{EtC} \equiv \mathrm{Mo}\left(\mathrm{OC}\left(\mathrm{CH}_{3}\right)\left(\mathrm{CF}_{3}\right)_{2}\right)_{3}(\mathrm{DME})(0.097 \mathrm{~g}, 0.126 \mathrm{mmol}, 0.10$ equiv $)$ in toluene $(10 \mathrm{~mL})$ were added. The stirred mixture was heated at $80^{\circ} \mathrm{C}$ for $16 \mathrm{~h}$, then the mixture was cooled to RT and quantitatively transferred to a $250 \mathrm{~mL}$ round-bottom flask with the aid of toluene $(2 \times 3 \mathrm{~mL})$. Silica gel $(15 \mathrm{~g})$ was added, volatile materials were removed from the suspension via rotary evaporation, and the resulting powder was layered onto a plug of silica gel $(50 \mathrm{~g})$ that had been equilibrated 
with $10 \% \mathrm{EtOAc}$ in $\mathrm{CH}_{2} \mathrm{Cl}_{2}$. The plug was flushed with the same solvent mixture until the yellow color was very faint ( $\sim 600 \mathrm{~mL})$. The volume of the filtrate was concentrated to $5-10 \mathrm{~mL}$ via rotary evaporation, then $\mathrm{MeOH}(100 \mathrm{~mL})$ was added. The precipitate was collected by filtration** and washed with $\mathrm{MeOH}(2 \times 25 \mathrm{~mL})$, affording $2(1.19 \mathrm{~g}, 77 \%)$ as a yellow solid.

Notes for Procedure \#3: *See general section (above) for important details on the source of the molecular sieves; ${ }^{* *}$ The precipitate was very fine, and filtration was greatly aided by celite. After filtration, the celite was extracted with $\mathrm{CH}_{2} \mathrm{Cl}_{2}$ for recovery of product (see the section titled "general notes on purification").

Characterization: ${ }^{1} \mathrm{H}$ NMR (benzene- $\left.d_{6}, 500 \mathrm{MHz}\right): \delta=10.38(\mathrm{~s}, 4 \mathrm{H}), 10.20(\mathrm{~s}, 4 \mathrm{H}), 9.46(\mathrm{~s}, 4 \mathrm{H})$, $9.46(\mathrm{~s}, 4 \mathrm{H}), 9.16(\mathrm{~d}, J=8.6 \mathrm{~Hz}, 4 \mathrm{H}), 9.01(\mathrm{~d}, J=1.7 \mathrm{~Hz}, 4 \mathrm{H}), 8.27$ (dd, $J=8.2,1.8 \mathrm{~Hz}, 4 \mathrm{H}), 4.94$ $(\mathrm{s}, 8 \mathrm{H}), 4.93(\mathrm{~s}, 8 \mathrm{H}), 3.97(\mathrm{~s}, 12 \mathrm{H}), 3.92(\mathrm{~s}, 12 \mathrm{H}), 3.78-3.68(\mathrm{~m}, 16 \mathrm{H}), 3.73(\mathrm{~s}, 12 \mathrm{H}), 3.41(\mathrm{~s}$, $12 \mathrm{H}), 3.41(\mathrm{~s}, 12 \mathrm{H}), 2.28-2.11(\mathrm{~m}, 16 \mathrm{H}), 1.11(\mathrm{t}, J=7.3 \mathrm{~Hz}, 12 \mathrm{H}), 1.07(\mathrm{t}, J=7.3 \mathrm{~Hz}, 12 \mathrm{H})$; ${ }^{13} \mathrm{C}\left\{{ }^{1} \mathrm{H}\right\}$ NMR (chloroform-d, $\left.176 \mathrm{MHz}\right): \delta=144.83,144.42,143.70,136.76,136.72,136.21$, 136.14, 134.58, 134.43, 131.29, 131.20, 130.11, 129.90, 129.87, 129.83, 128.57, 128.42, 128.22, 127.36, 127.17, 126.51, 122.77, 122.77, 122.41, 122.36, 117.94, 117.91, 90.26, 69.20, 69.19, $61.29,61.16,61.14,58.97,58.94,35.12$, 35.09, 25.92, 25.90, 14.71, 14.67; HRMS-MALDI-TOF (m/z): [M]+ calcd. for $\mathrm{C}_{164} \mathrm{H}_{160} \mathrm{O}_{20}, 2449.1503$; found, 2449.1709.

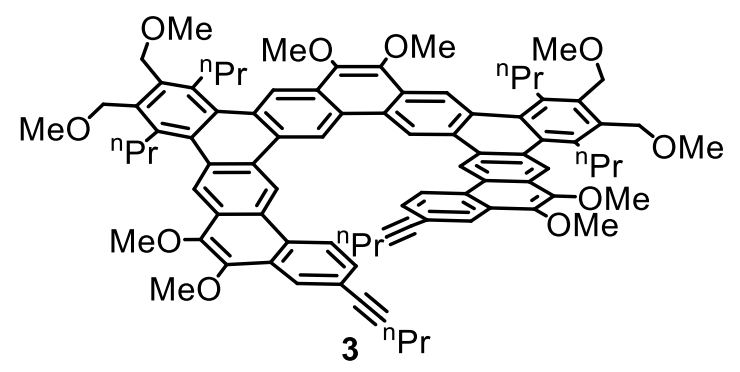

\section{Alkynylated expanded helicene (3)}

Procedure: To a stirred solution of $[\operatorname{Ir}(\mathrm{COD}) \mathrm{Cl}]_{2}(0.0133 \mathrm{~g}, 0.0199 \mathrm{mmol}, 0.02$ equiv) in benzene $(0.5 \mathrm{~mL})$ was added (dropwise over $\sim 1 \mathrm{~min})$ a solution of dppe $(0.0158 \mathrm{~g}, 0.0397 \mathrm{mmol}, 0.04$ equiv) in benzene $(0.5 \mathrm{~mL})$. This mixture was immediately added to a flask containing a solution of hexayne 6 ( $1.10 \mathrm{~g}, 0.993 \mathrm{mmol}, 1.0$ equiv) and 1,4-dimethoxy-2-butyne (0.45 g, $4.0 \mathrm{mmol}, 4.0$ equiv) in benzene $(19 \mathrm{~mL})$. The flask was sealed with a Teflon stopper and the reaction mixture was heated at $80{ }^{\circ} \mathrm{C}$ for $2 \mathrm{~h}$. The mixture was allowed to cool to RT and poured onto a fritted funnel packed with silica gel $(20 \mathrm{~g})$. The silica was eluted with $5 \% \mathrm{EtOAc}$ in $\mathrm{CH}_{2} \mathrm{Cl}_{2}(\sim 200 \mathrm{~mL}$; until the yellow color in the eluent was faint) and the filtrate was concentrated via rotary evaporation. To fully remove solvents, the residue was suspended in hexanes $(10 \mathrm{~mL})$ and sonicated, then solvents were removed via rotary evaporation. The residue was again suspended in hexanes $(40 \mathrm{~mL})$ and stirred vigorously until a fine, light-yellow suspension was obtained (sonication is also effective). The suspension was filtered and the solid was washed with thoroughly with hexanes $(2 \times 10 \mathrm{~mL})$. Residual solvents were removed under high vacuum to afford expanded helicene $3(1.07 \mathrm{~g}, 80 \%)$ as a light-yellow solid. When this reaction was run on a smaller scale in benzene- $d_{6}$ solvent and monitored by ${ }^{1} \mathrm{H}$ NMR spectroscopy $(1,3,5-$ trimethoxybenzene internal standard), 3 was observed in $88 \%$ yield.

Characterization: ${ }^{1} \mathrm{H}$ NMR (chloroform- $\left.d, 600 \mathrm{MHz}\right): \delta=10.23(\mathrm{~s}, 2 \mathrm{H}), 10.05(\mathrm{~s}, 2 \mathrm{H}), 9.10(\mathrm{~d}, \mathrm{~J}=$ $8.5 \mathrm{~Hz}, 2 \mathrm{H}), 9.05(\mathrm{~s}, 2 \mathrm{H}), 9.03(\mathrm{~s}, 2 \mathrm{H}), 8.38(\mathrm{~d}, J=1.7 \mathrm{~Hz}, 2 \mathrm{H}), 7.63(\mathrm{dd}, J=8.5,1.7 \mathrm{~Hz}, 2 \mathrm{H})$, $4.85(\mathrm{~s}, 4 \mathrm{H}), 4.85(\mathrm{~s}, 4 \mathrm{H}), 4.22(\mathrm{~s}, 6 \mathrm{H}), 4.20(\mathrm{~s}, 6 \mathrm{H}), 4.19(\mathrm{~s}, 6 \mathrm{H}), 3.62(\mathrm{~s}, 6 \mathrm{H}), 3.62(\mathrm{~s}, 6 \mathrm{H}), 3.57$ $-3.49(\mathrm{~m}, 8 \mathrm{H}), 2.50(\mathrm{t}, J=7.1 \mathrm{~Hz}, 4 \mathrm{H}), 2.07-1.96(\mathrm{~m}, 8 \mathrm{H}), 1.80-1.68(\mathrm{~m}, 4 \mathrm{H}), 1.12(\mathrm{t}, J=7.4$ $\mathrm{Hz}, 6 \mathrm{H}$ ), 1.07 (t, $J=7.3 \mathrm{~Hz}, 6 \mathrm{H}), 1.05(\mathrm{t}, J=7.3 \mathrm{~Hz}, 6 \mathrm{H}) ;{ }^{3} \mathrm{C}\left\{{ }^{1} \mathrm{H}\right\} \mathrm{NMR}$ (chloroform-d, $151 \mathrm{MHz}$ ): $\delta=144.67,144.29,143.62,136.60,136.57,136.15,136.08,134.54,134.52,131.23,131.18$, 
$130.13,130.01,129.97,129.67,128.45,127.97,127.93,127.40,127.23,125.65,123.24,122.81$, 122.75, 122.34, 117.86, 117.84, 91.30, 81.30, 69.19, 69.20, 61.22, 61.16, 61.11, 58.95, 58.93, 35.10, 34.99, 25.89, 25.87, 22.50, 21.86, 14.68, 14.67, 13.89; HRMS-MALDI-TOF (m/z): [M] ${ }^{+}$ calcd. for $\mathrm{C}_{90} \mathrm{H}_{94} \mathrm{O}_{10}, 1334.6847$; found, 1334.6479 .<smiles>COc1c2cc(Br)c(C#C[Tl])cc2c(OC)c2cc(C#CC#P)c(Br)cc12</smiles>

Large scale prep of 3,6-dibromo-2,7-bis(pent-1-ynyl)-9,10-dimethoxyphenanthrene (4) Procedure: This is an adaptation of the previously reported procedure. ${ }^{7}$ The only major difference is the scale, which required a modification of the reaction setup since the starting material $(3,6$ dibromo-2,7-diiodo-9,10-dimethoxyphenanthrene) is a highly flocculent solid that is difficult to load into the narrow neck of a Teflon-stoppered flask. A $2000 \mathrm{~mL}$ round-bottom Schlenk flask was charged with 3,6-dibromo-2,7-diiodo-9,10-dimethoxyphenanthrene (60.2 g, $92.9 \mathrm{mmol}, 1.0$ equiv), $\mathrm{Pd}\left(\mathrm{PPh}_{3}\right)_{4}$ (2.69 g, $2.32 \mathrm{mmol}, 0.025$ equiv), and Cul (1.06 g, $5.58 \mathrm{mmol}, 0.06$ equiv). The flask sealed with a rubber septum and subjected to three vacuum / $\mathrm{N}_{2}$ cycles, then THF (580 mL) and triethylamine $\left(290 \mathrm{~mL}\right.$ ) (both of which had been thoroughly sparged with $\mathrm{N}_{2}$ ) were added via cannula transfer. To this mixture was added 1-pentyne $(22.9 \mathrm{~mL}, 232 \mathrm{mmol}, 2.5$ equiv) via syringe, then (immediately after this addition) the septum was rapidly replaced with an efficient reflux condenser against $\mathrm{N}_{2}$ flow. The mixture was heated at $70^{\circ} \mathrm{C}$ under $\mathrm{N}_{2}$ for $18 \mathrm{~h}$, then brought to RT and poured into ice-cold aqueous $\mathrm{HCl}(3 \mathrm{M}, 1300 \mathrm{~mL})$. The mixture was extracted with $\mathrm{CH}_{2} \mathrm{Cl}_{2}$ $(800 \mathrm{~mL})$ and the extract was dried with $\mathrm{MgSO}_{4}$ and filtered. Solvents were removed from the filtrate via rotary evaporation, then the crude product was dissolved in $\mathrm{CH}_{2} \mathrm{Cl}_{2}(300 \mathrm{~mL})$ and this solution was diluted with hexanes $(800 \mathrm{~mL})$. The solution was eluted through a plug* of silica gel $(200 \mathrm{~g})$, and the plug was flushed with 3:1 hexanes: $\mathrm{CH}_{2} \mathrm{Cl}_{2}(\sim 2000 \mathrm{~mL}$, or until no more desired product was seen by TLC analysis). Solvents were removed via rotary evaporation and further purification was carried out as follows (repeated $2 x$ ). The solid was dissolved in $\mathrm{CH}_{2} \mathrm{Cl}_{2}(300 \mathrm{~mL})$, then $\mathrm{MeOH}(600 \mathrm{~mL})$ was added to produce an immediate precipitate that was collected by filtration and washed with $\mathrm{MeOH}(200 \mathrm{~mL})$. The yield of 4, after drying under high vacuum at 60$70{ }^{\circ} \mathrm{C}$ for $1 \mathrm{~h},{ }^{* *}$ was $35 \mathrm{~g}(71 \%)$, as an off-white solid.

Notes: *The silica plug was set up like a standard silica column (i.e. it was thoroughly equilibrated before elution), but a single fraction was collected. ${ }^{*}$ The ${ }^{1} \mathrm{H}$ NMR spectrum should be examined for the absence of $\mathrm{CH}_{2} \mathrm{Cl}_{2}$ and $\mathrm{MeOH}$.

Characterization: ${ }^{1} \mathrm{H}$ NMR data matches that reported in the literature. ${ }^{7}$

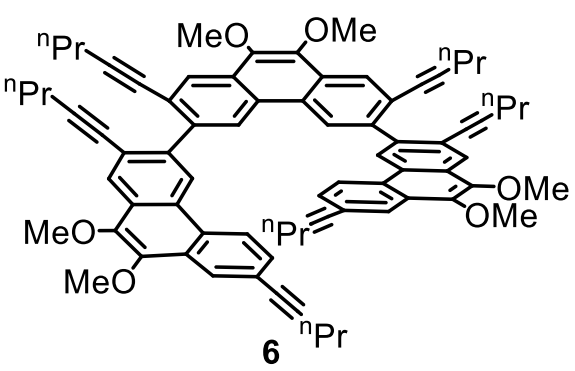

\section{Hexa(pentynyl)terphenanthrene (6)}

Procedure: A $50 \mathrm{~mL}$ flask equipped with Teflon stopper was charged with monobromide 5 (1.60 $\mathrm{g}, 3.56 \mathrm{mmol}^{*}, 4.0$ equiv) and THF $(3.0 \mathrm{~mL})$, and to this solution was added ${ }^{\mathrm{PrMgCl}} \cdot \mathrm{LiCl}(0.81 \mathrm{M}$ 
in THF, $4.06 \mathrm{~mL}, 3.29 \mathrm{mmol}, 3.7$ equiv) via syringe. The flask was sealed, and the mixture was heated at $60{ }^{\circ} \mathrm{C}$ for $3 \mathrm{~h}$. A $1 \mathrm{~mL}$ aliquot was analyzed by ${ }^{1} \mathrm{H}$ NMR spectroscopy ${ }^{\star *}$ to ensure complete consumption of $\mathrm{irMgCl} \cdot \mathrm{LiCl}$, then the aliquot was recombined with the original solution. To this solution was added $\mathrm{PdCl}_{2}(\mathrm{IPr})$ (3-chloropyridine) $(0.018 \mathrm{~g}, 0.027 \mathrm{mmol}, 0.03$ equiv) in THF ( $2 \mathrm{~mL})$, followed by dibromide $4(0.470 \mathrm{~g}, 0.890 \mathrm{mmol}, 1.0$ equiv). The resulting homogeneous mixture was allowed to stand at RT $\left(\sim 21^{\circ} \mathrm{C}\right)$ for $20 \mathrm{~h}$, then quenched with saturated aqueous $\mathrm{NH}_{4} \mathrm{Cl}(20 \mathrm{~mL})$ and extracted with EtOAc $(30 \mathrm{~mL}$, then $10 \mathrm{~mL})$. The combined organic extracts were washed with saturated aqueous $\mathrm{NaCl}(20 \mathrm{~mL})$, dried with $\mathrm{MgSO}_{4}$, filtered, and the filtrate was concentrated under reduced pressure. The residue was purified by column chromatography (mass of silica: $120 \mathrm{~g}$; eluant: $25-33 \% \mathrm{CH}_{2} \mathrm{Cl}_{2}$ in hexanes) to afford hexayne $6(0.69 \mathrm{~g}, 70 \%)$ as a pale-yellow solid.

Notes: *This compound was used as an inseparable mixture with its dibromide precursor (compound 4) and the fully dibrominated side product. To simplify stoichiometry calculations, it was assumed to be pure. See the previous report for more details. ${ }^{8 *}$ Hoye's No-D NMR method ${ }^{11}$ was used.

Characterization: ${ }^{1} \mathrm{H}$ NMR (chloroform-d, $400 \mathrm{MHz}$ ): $\delta 8.74(\mathrm{~s}, 2 \mathrm{H}), 8.69(\mathrm{~s}, 2 \mathrm{H}), 8.44(\mathrm{~d}, J=8.7$ $\mathrm{Hz}, 2 \mathrm{H}), 8.42(\mathrm{~s}, 2 \mathrm{H}), 8.32(\mathrm{~s}, 2 \mathrm{H}), 8.24(\mathrm{~d}, J=1.7 \mathrm{~Hz}, 2 \mathrm{H}), 7.53(\mathrm{dd}, J=8.5,1.8 \mathrm{~Hz}, 2 \mathrm{H}), 4.18$ $(\mathrm{s}, 6 \mathrm{H}), 4.09(\mathrm{~s}, 6 \mathrm{H}), 4.07(\mathrm{~s}, 6 \mathrm{H}), 2.45(\mathrm{t}, J=7.1 \mathrm{~Hz}, 4 \mathrm{H}), 2.17(\mathrm{t}, J=6.9 \mathrm{~Hz}, 4 \mathrm{H}), 2.12(\mathrm{t}, J=6.9$ $\mathrm{Hz}, 4 \mathrm{H}$ ), 1.68 (sext, $J=7.2 \mathrm{~Hz}, 4 \mathrm{H}$ ), 1.30 (sext, $J=7.2 \mathrm{~Hz}, 4 \mathrm{H}$ ), 1.25 (sext, $J=7.2 \mathrm{~Hz}, 4 \mathrm{H}$ ), 1.08 $(\mathrm{t}, J=7.3 \mathrm{~Hz}, 6 \mathrm{H}), 0.67(\mathrm{t}, J=7.3 \mathrm{~Hz}, 6 \mathrm{H}), 0.59(\mathrm{t}, J=7.3 \mathrm{~Hz}, 6 \mathrm{H}) ;{ }^{13} \mathrm{C}\left\{{ }^{1} \mathrm{H}\right\}$ NMR (chloroform- $d$, $101 \mathrm{MHz}): \delta=143.89,143.89,143.84,140.44,140.30,129.36,128.95,128.54,128.30,127.68$, 127.28, 127.04, 126.18, 126.13, 125.43, 125.07, 124.81, 123.06, 123.04, 122.99, 122.64, 94.45, $94.42,91.34,81.27,80.73,80.67,61.27,61.18,61.14,22.39,22.04,22.03,21.70,21.64,21.64$, 13.78, 13.35, 13.32; HRMS-MALDI-TOF (m/z): [M] ${ }^{+}$calcd. for $\mathrm{C}_{78} \mathrm{H}_{74} \mathrm{O}_{6}, 1106.549$; found, 1106.511. 


\section{${ }^{1} \mathrm{H}$ and ${ }^{13} \mathrm{C}\left\{{ }^{1} \mathrm{H}\right\}$ NMR spectra}

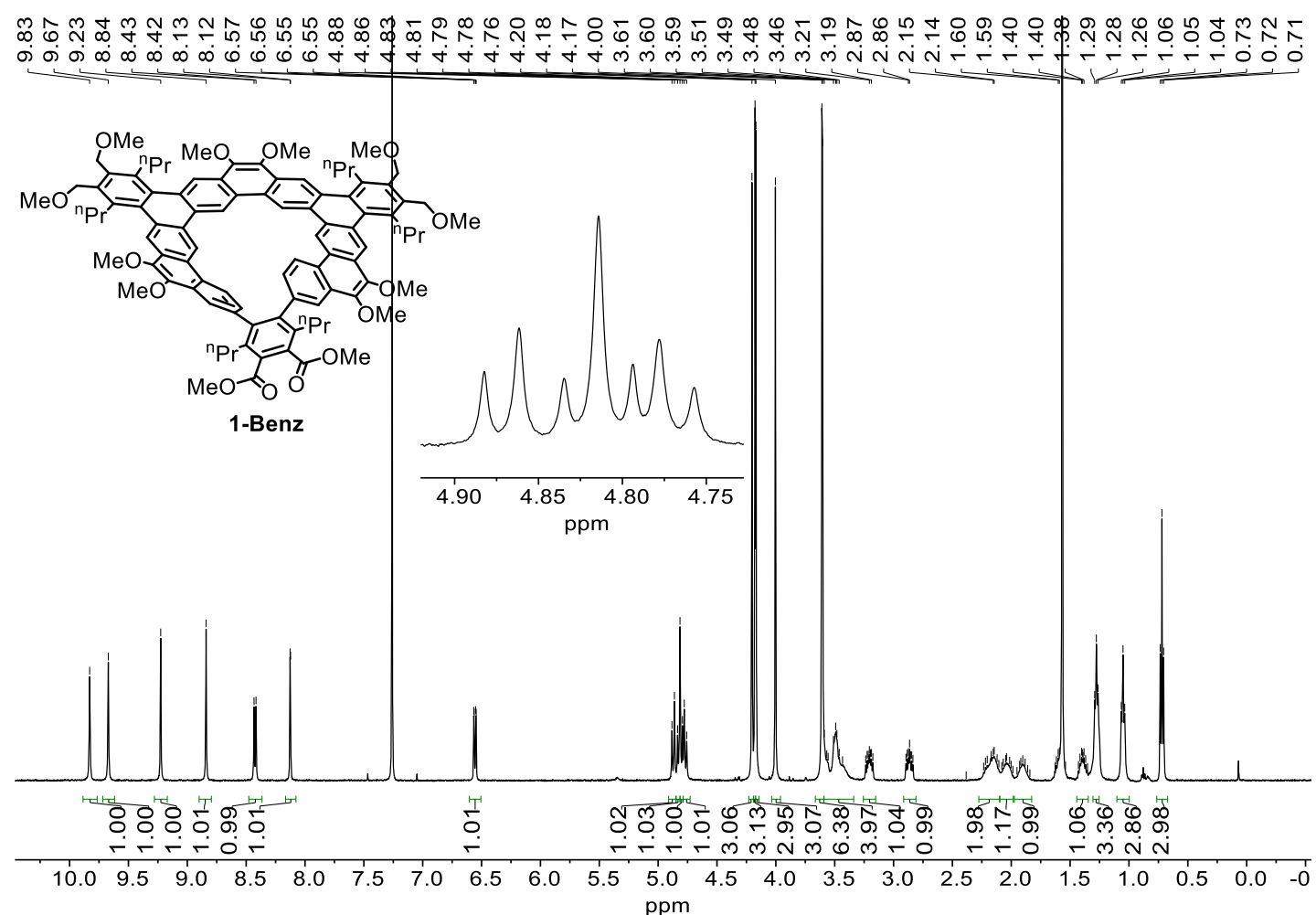

Figure S1. ${ }^{1} \mathrm{H}$ NMR Spectrum (500 MHz, chloroform- $d$, [c] = $2.6 \mathrm{mM}$ ) of 1-Benz.

อ

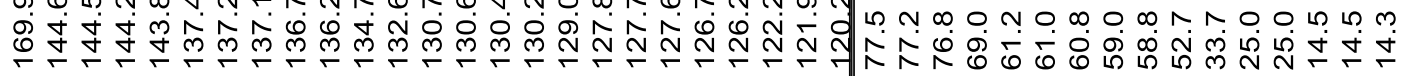
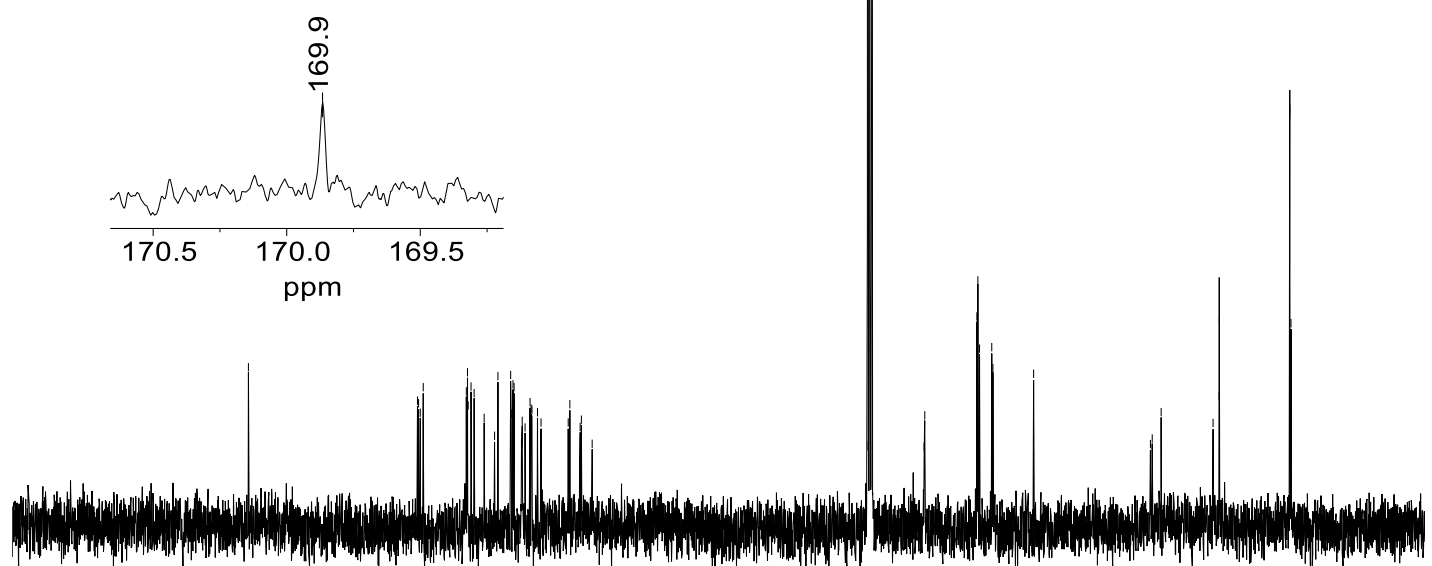

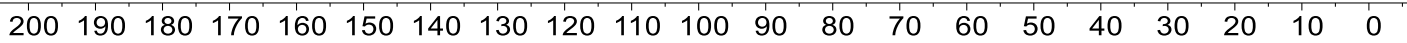
ppm

Figure S2. ${ }^{13} \mathrm{C}\left\{{ }^{1} \mathrm{H}\right\}$ NMR Spectrum (101 MHz, chloroform- $d$ ) of 1-Benz. 


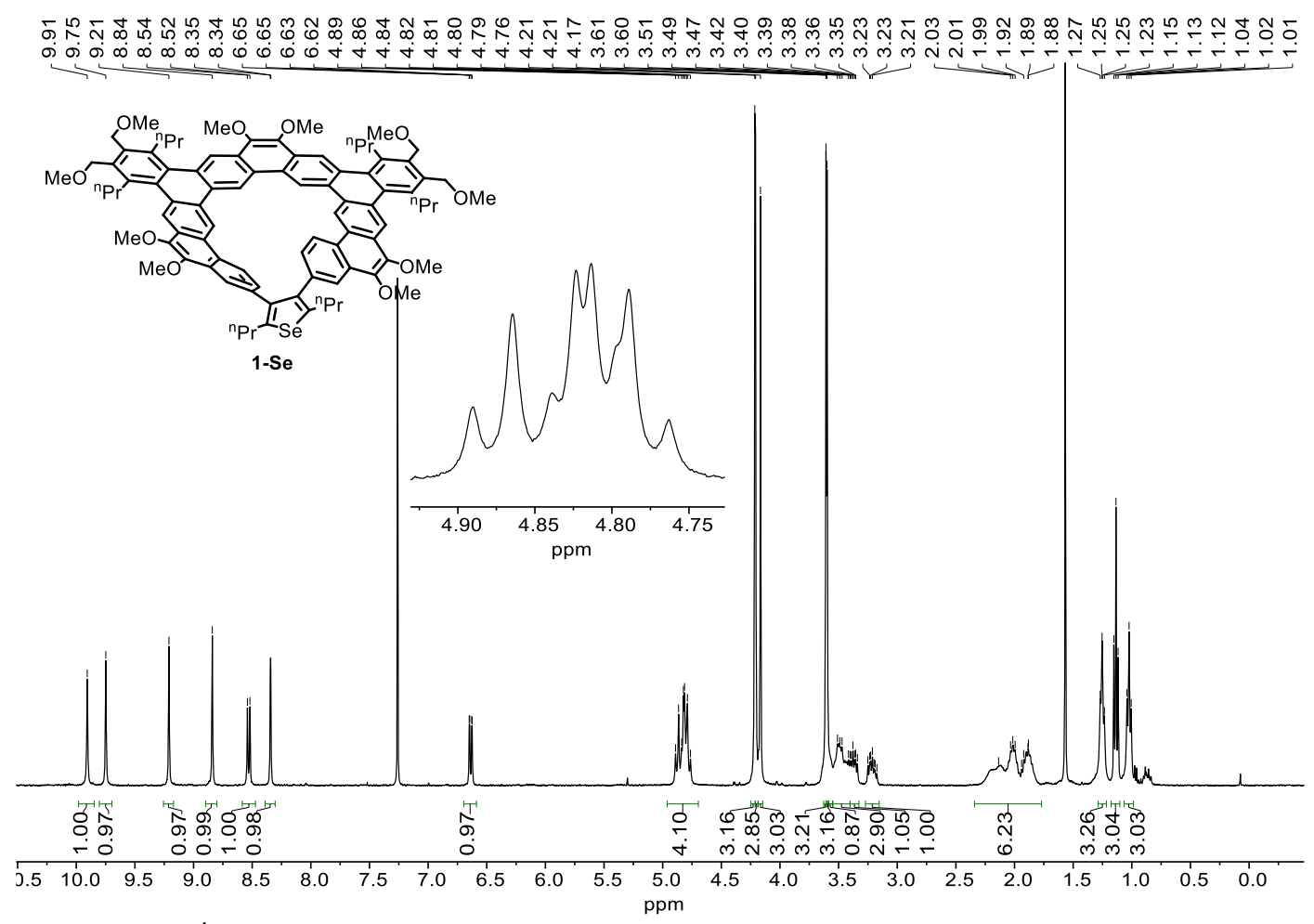

Figure S3. ${ }^{1} \mathrm{H}$ NMR Spectrum (400 MHz, chloroform- $d$, [c] = $6.2 \mathrm{mM}$ ) of 1-Se.

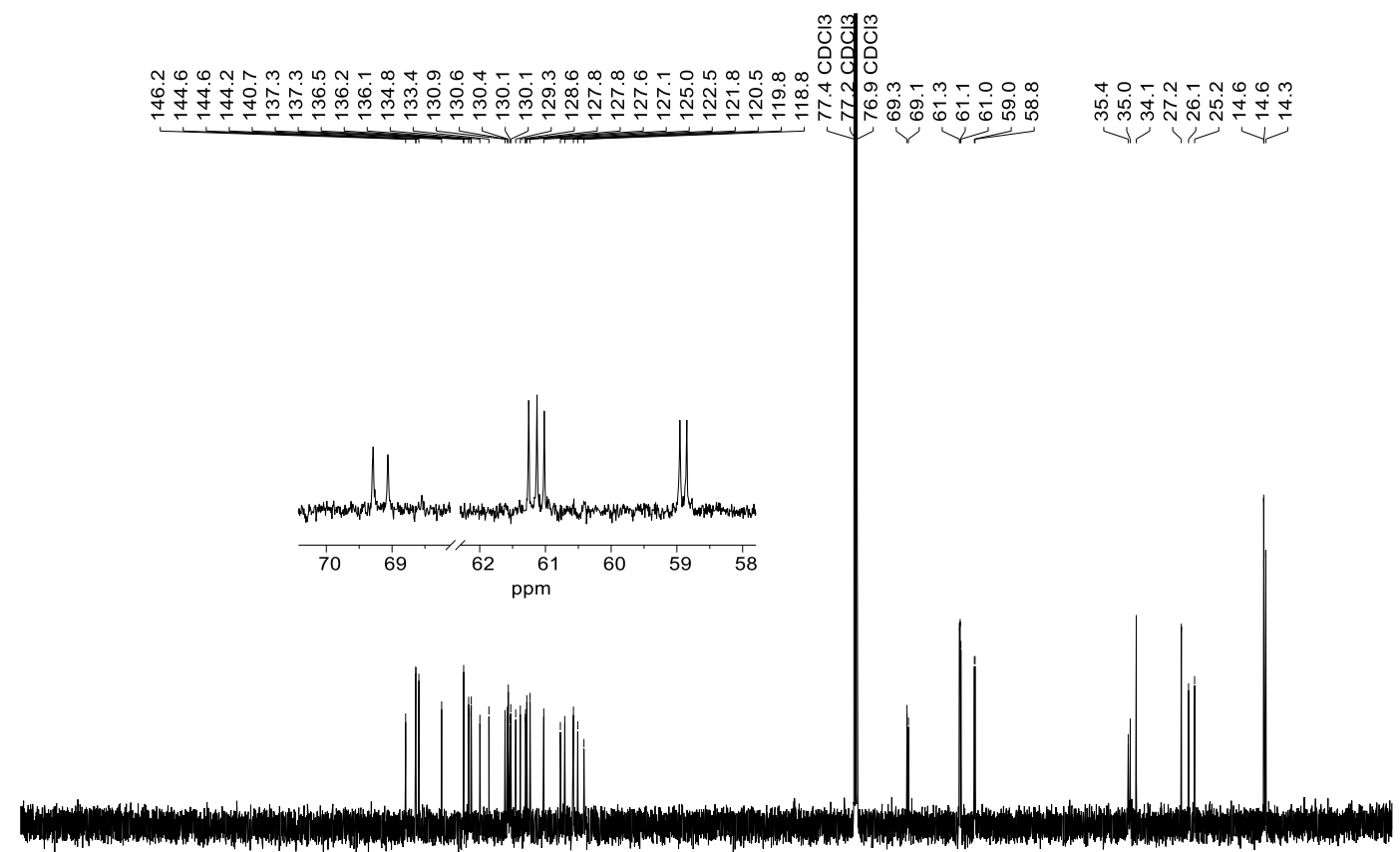

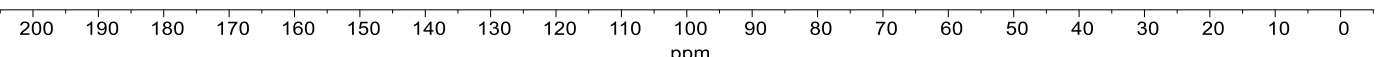

Figure S4. ${ }^{13} \mathrm{C}\left\{{ }^{1} \mathrm{H}\right\}$ NMR Spectrum (151 MHz, chloroform- $d$ ) of 1-Se. 


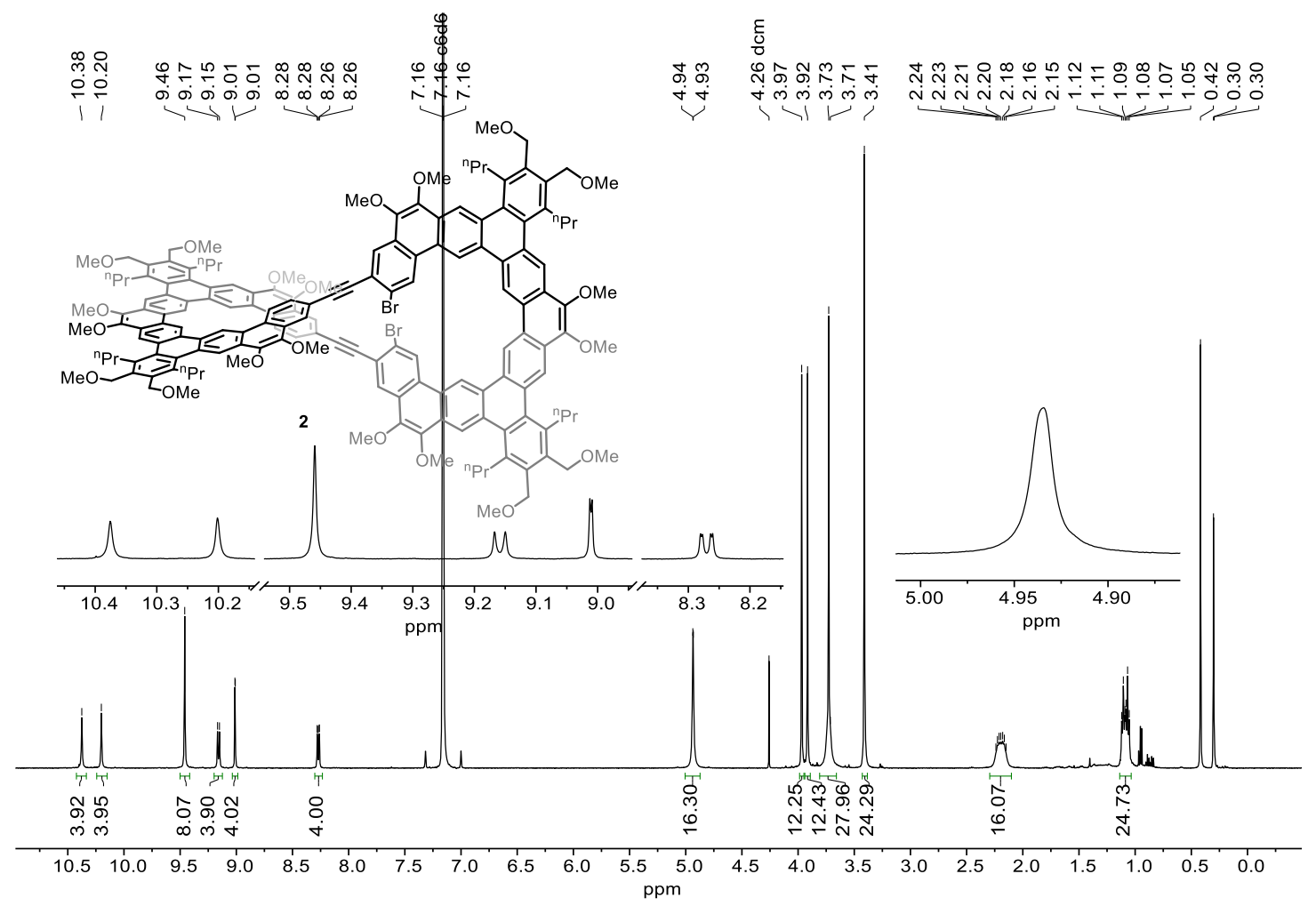

Figure S5. ${ }^{1} \mathrm{H}$ NMR Spectrum (500 MHz, benzene- $\left.d_{6}\right)$ of 2.

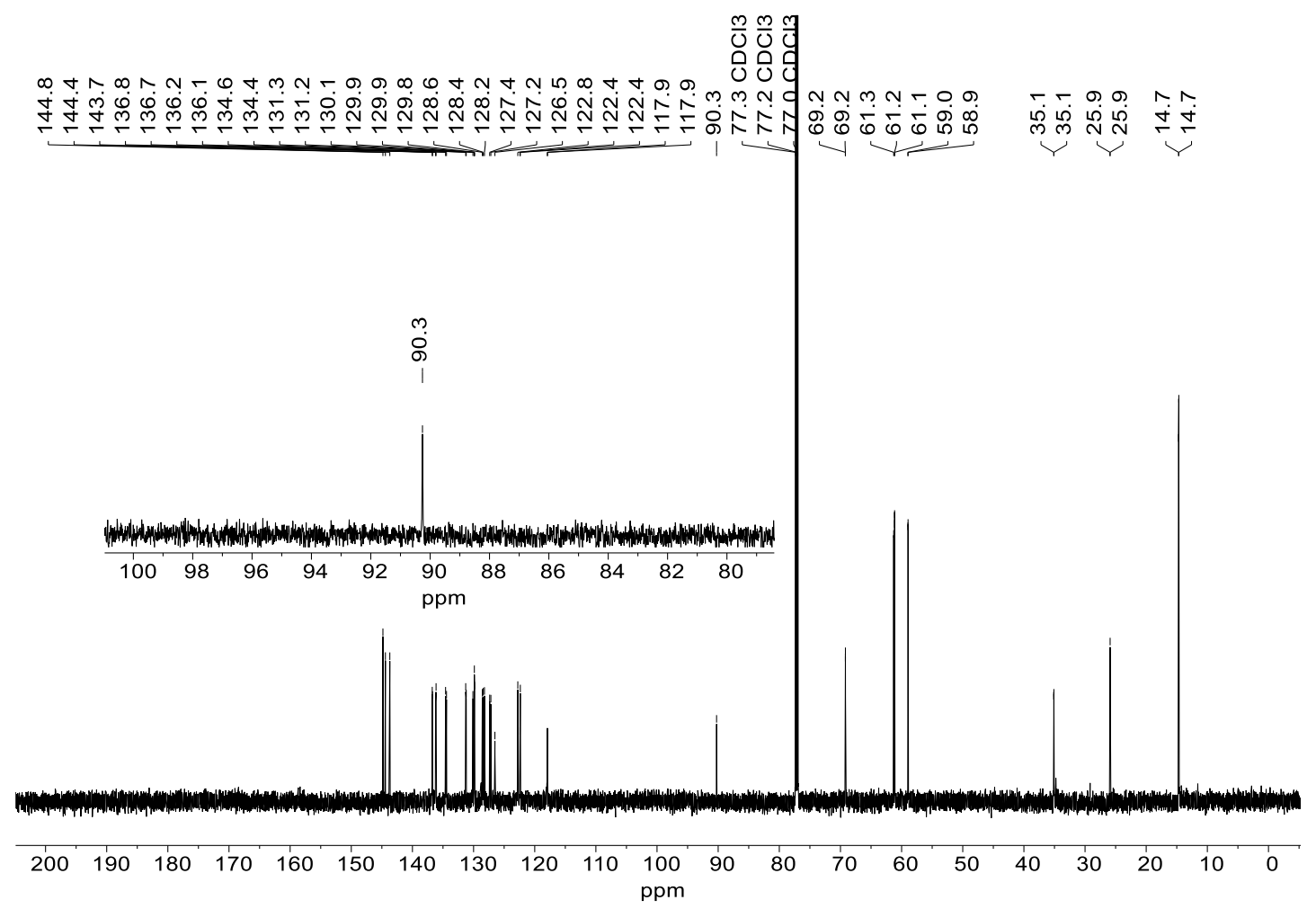

Figure S6. ${ }^{13} \mathrm{C}\left\{{ }^{1} \mathrm{H}\right\}$ NMR Spectrum (176 MHz, chloroform- $d$ ) of 2. 


\section{(a)}

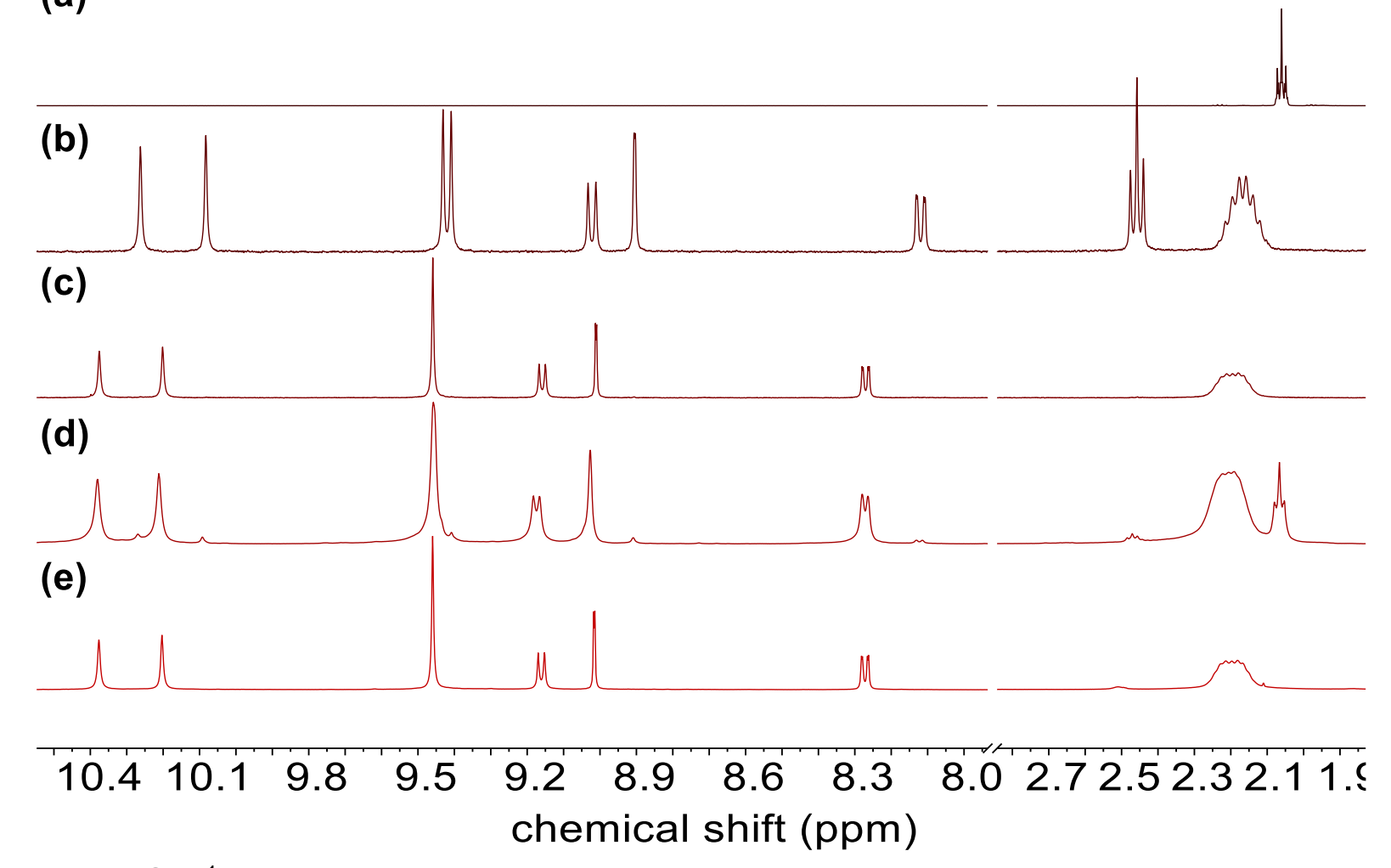

Figure S7. ${ }^{1} \mathrm{H}$ NMR monitoring of the macrocyclization of alkynylated expanded helicene 3 to form figure-eight 2. Shown in this Figure are the partial ${ }^{1} \mathrm{H}$ NMR spectra (500 MHz, benzene- $d_{6}$ ) of (a) 4-octyne; (b) starting expanded helicene 3; (c) purified figure-eight 2; (d) reaction mixture after treatment of 3 with $\mathrm{EtC} \equiv \mathrm{Mo}\left(\mathrm{OC}\left(\mathrm{CH}_{3}\right)\left(\mathrm{CF}_{3}\right)_{2}\right)_{3}(\mathrm{DME})(10 \mathrm{~mol} \%)$ for $16 \mathrm{~h}$ at $80{ }^{\circ} \mathrm{C}$ (no $5 \AA$ molecular sieves); (e) reaction mixture after treatment of $\mathbf{3}$ with $\mathrm{EtC} \equiv \mathrm{Mo}\left(\mathrm{OC}\left(\mathrm{CH}_{3}\right)\left(\mathrm{CF}_{3}\right)_{2}\right)_{3}(\mathrm{DME})$ (10 mol\%) for $16 \mathrm{~h}$ at $80^{\circ} \mathrm{C}$ in the presence of $5 \AA$ molecular sieves. Notably, no helicene 3 or byproduct 4-octyne are observed in spectrum (e). 


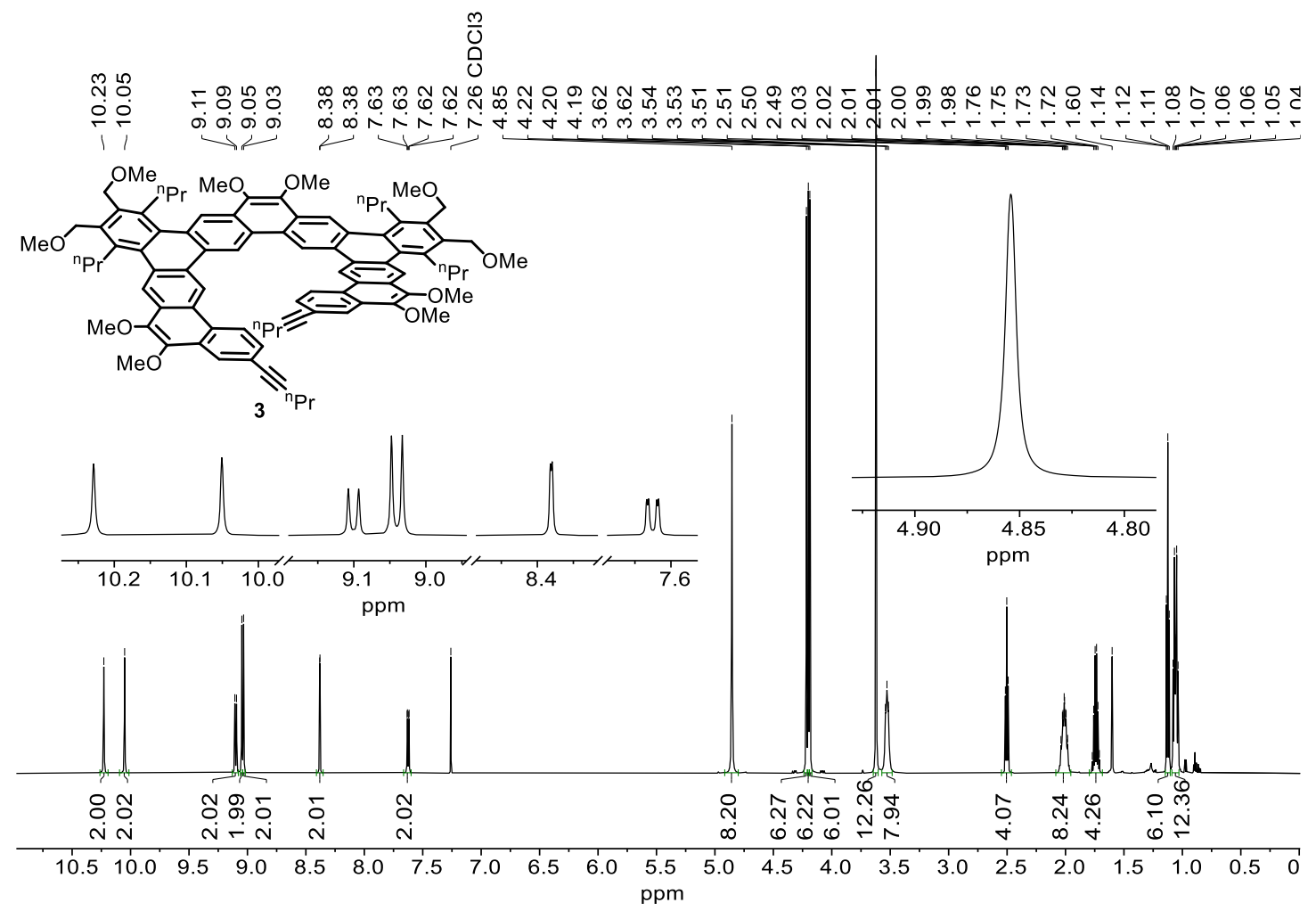

Figure S8. ${ }^{1} \mathrm{H}$ NMR Spectrum $(600 \mathrm{MHz}$, chloroform- $d$ ) of 3.

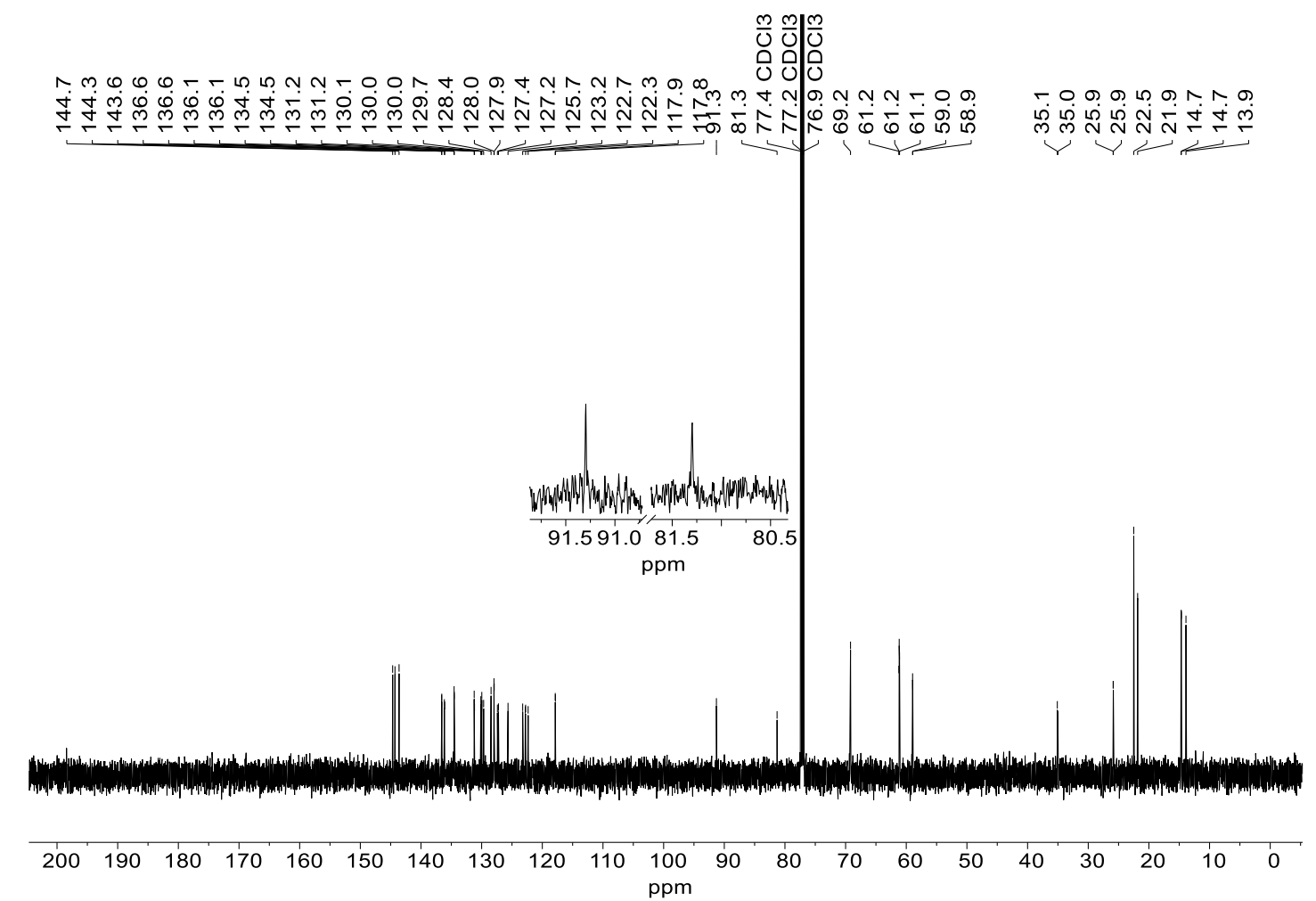

Figure S9. ${ }^{13} \mathrm{C}\left\{{ }^{1} \mathrm{H}\right\}$ NMR Spectrum (151 MHz, chloroform-d) of 3 . 


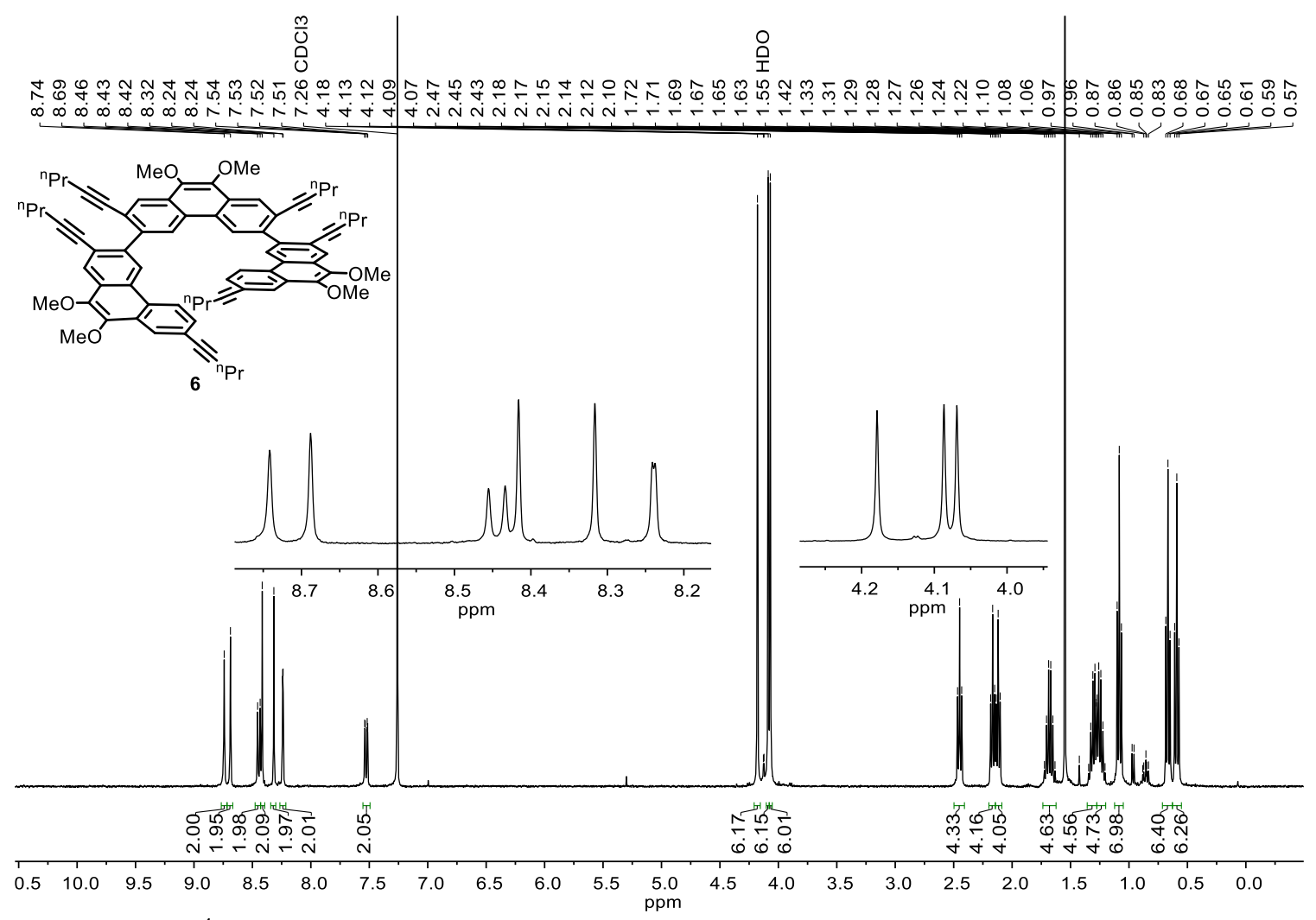

Figure S10. ${ }^{1} \mathrm{H}$ NMR Spectrum (400 MHz, chloroform- $d$ ) of 6.

$\frac{m}{\mathrm{O}} \frac{\mathrm{m}}{\mathrm{O}} \mathrm{m}$

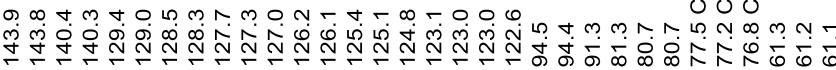

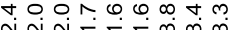

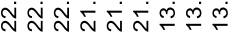

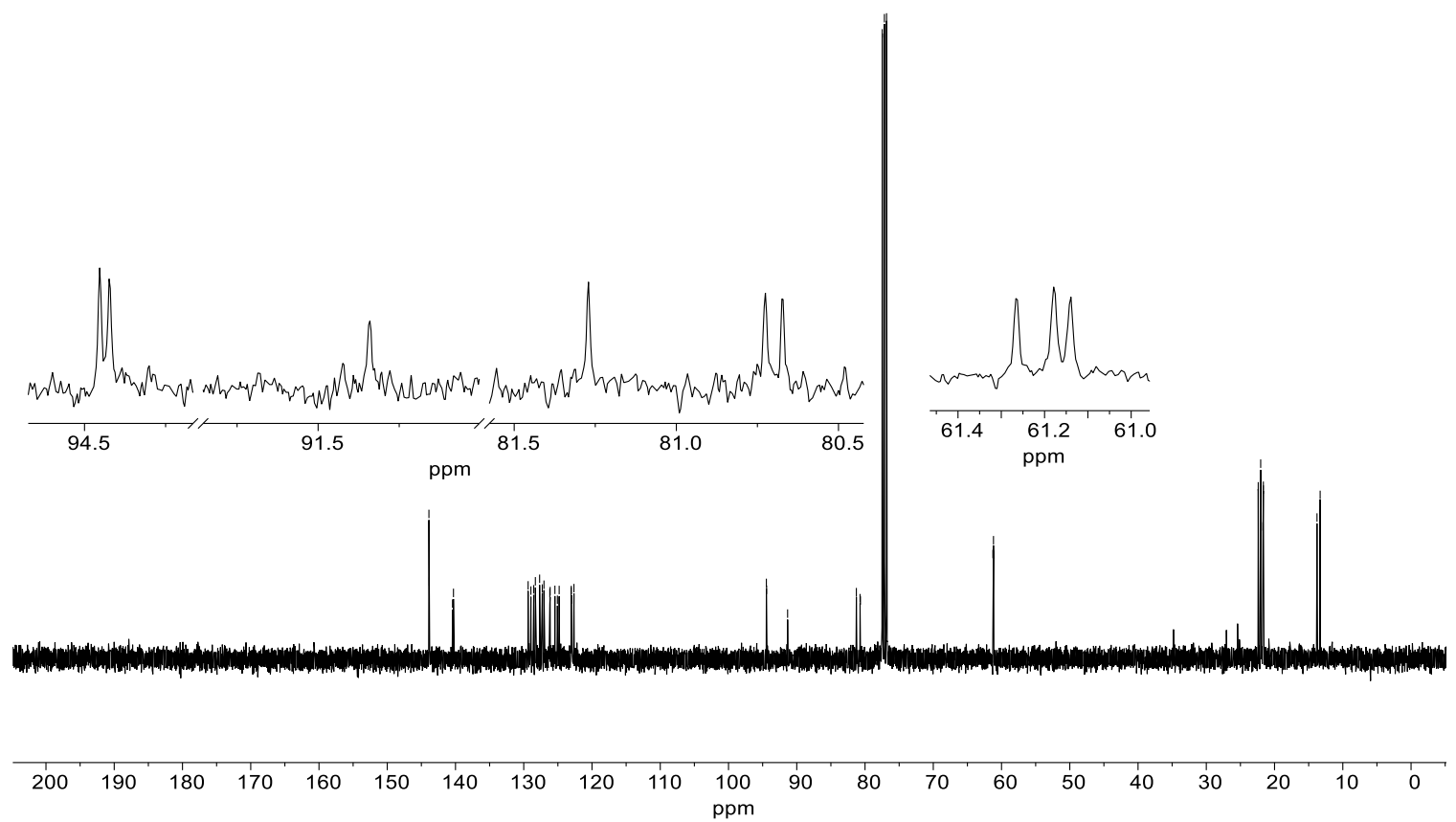

Figure S11. ${ }^{13} \mathrm{C}\left\{{ }^{1} \mathrm{H}\right\}$ NMR Spectrum (101 MHz, chloroform-d) of 6. 


\section{Variable concentration ${ }^{1} \mathrm{H}$ NMR spectra}

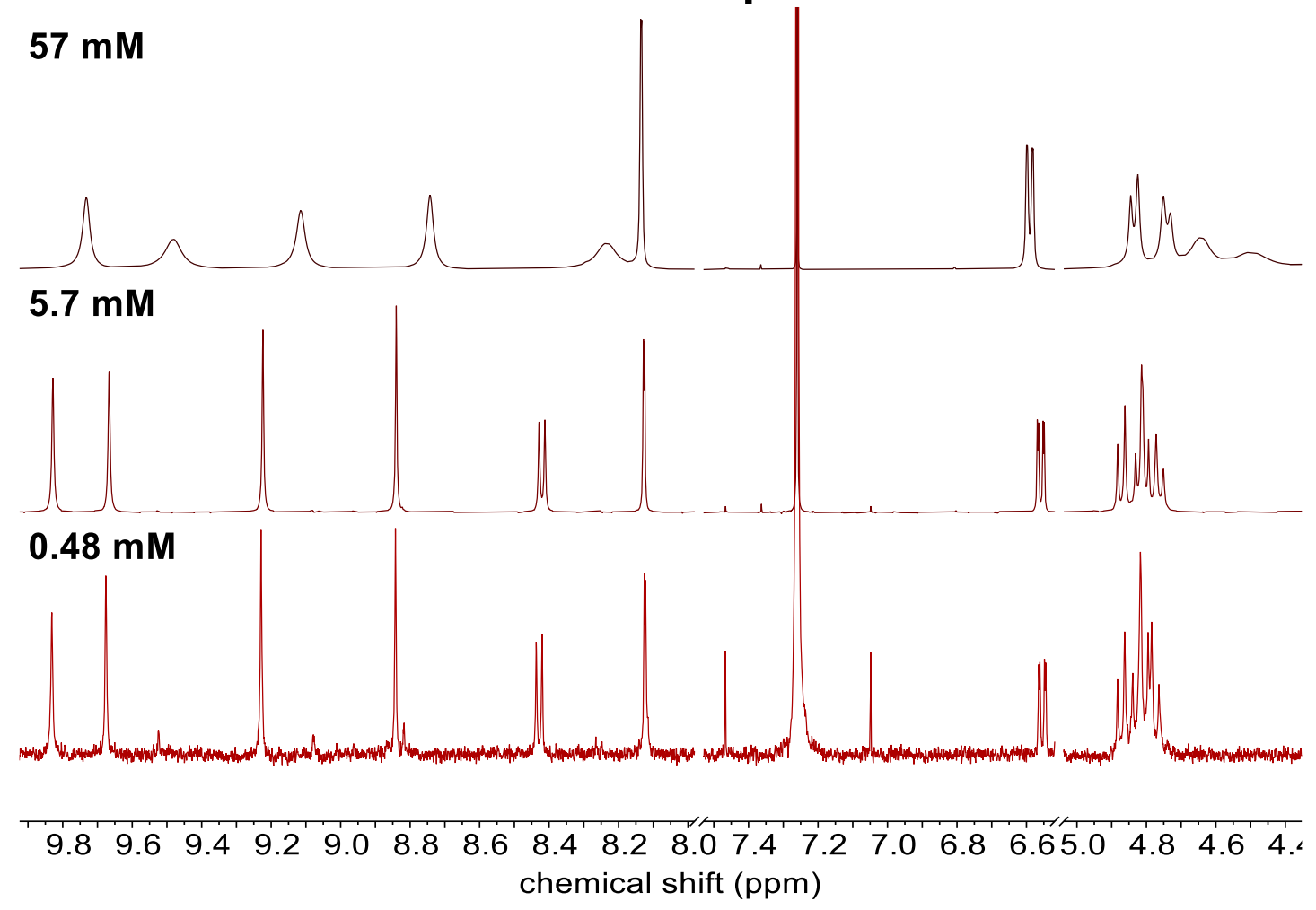

Figure S12. Variable concentration partial ${ }^{1} \mathrm{H}$ NMR spectra $(500 \mathrm{MHz}$, chloroform- $d$ ) of 1-Benz.
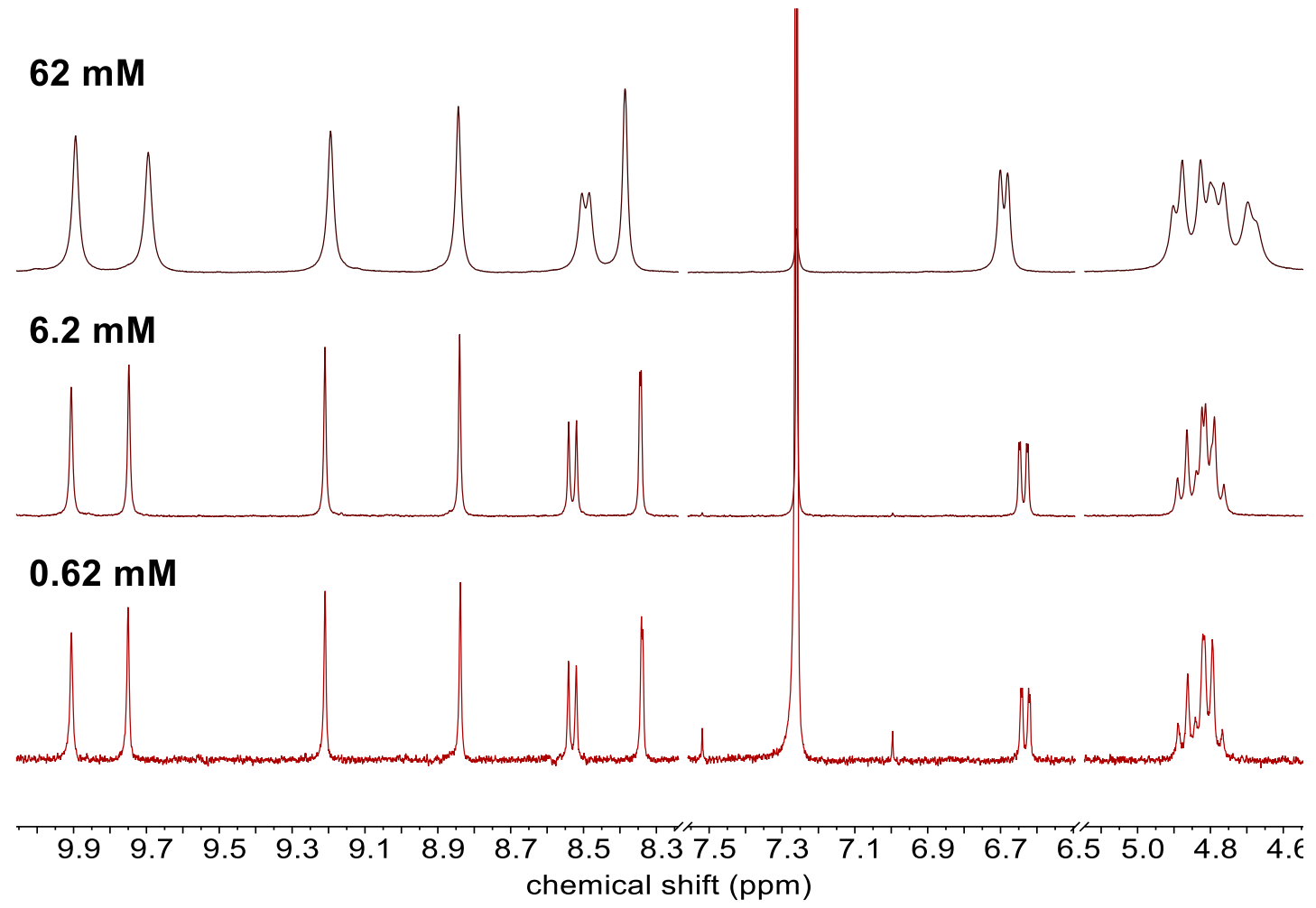

Figure S13. Variable concentration partial ${ }^{1} \mathrm{H}$ NMR spectra (400 MHz, chloroform- $d$ ) of 1-Se. 

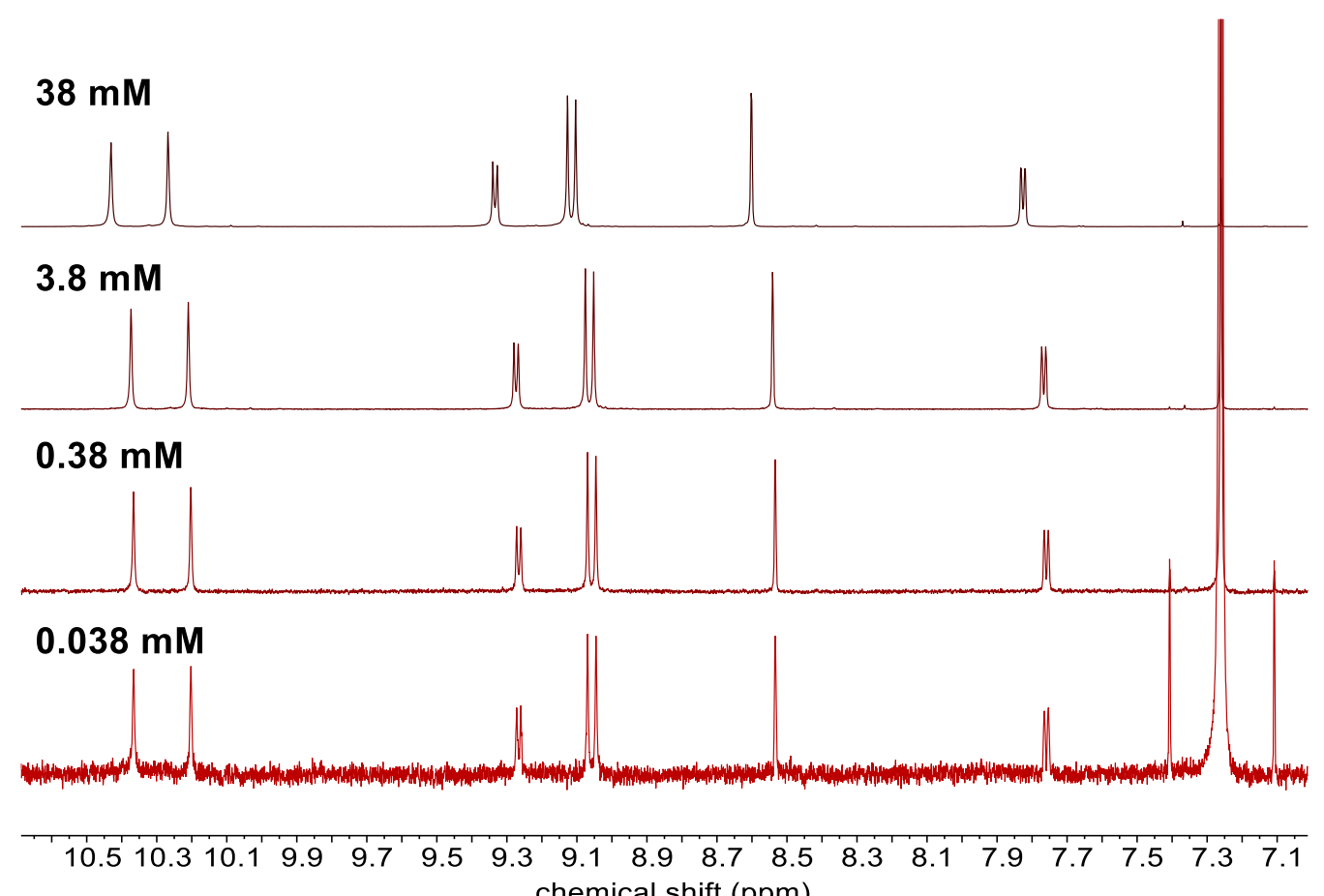

Figure S14. Variable concentration partial ${ }^{1} \mathrm{H}$ NMR spectra (700 MHz, chloroform- $d$ ) of 2.

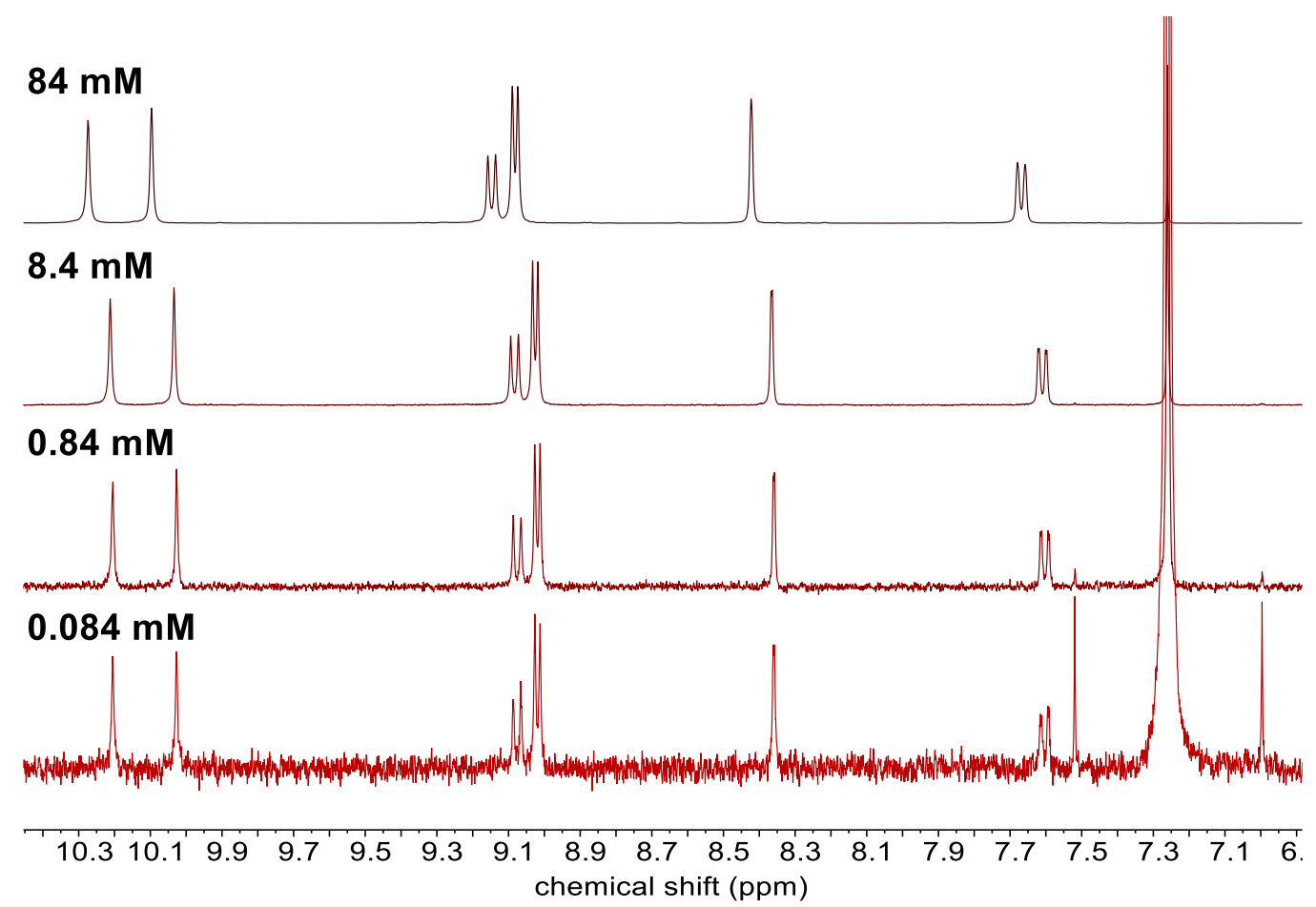

Figure S15. Variable concentration partial ${ }^{1} \mathrm{H}$ NMR spectra (400 MHz, chloroform- $d$ ) of 3. 


\section{Determination of enantiomerization barriers via dynamic ${ }^{1} \mathrm{H}$ NMR spectroscopy}

Enantiomerization free energy barriers $\left(\Delta G^{\ddagger}\right)$ were initially probed using variable temperature ${ }^{1} \mathrm{H}$ NMR spectroscopy by observation of the diastereotopic methylene protons on the peripheral methoxymethyl groups. For each compound (1-Se, 1-Benz, 2, and 3), two singlets are expected in the fast exchange regime and four doublets in the slow exchange regime. This ideal was only observed for only one compound, 1-Se. For the other three compounds, the coalescence temperature was outside of the operable solvent temperature range (for $\mathbf{2}$ and $\mathbf{3}$, this was due to apparent aggregation or viscosity issues).

Toluene- $d_{8}$ was chosen as solvent for these studies for the following reasons: 1) all of the new compounds are soluble; 2 ) the permitted temperature range is wide (approximately -95 to 110 ${ }^{\circ} \mathrm{C}$ ); and 3) the chemical shift separation for the methylene protons in the slow exchange regime is large relative to two other tested solvents (see Figure S16).

Temperatures below RT were calibrated with $\mathrm{MeOH}(4 \%) / \mathrm{MeOD}(96 \%)$ and those above RT with $80 \%$ ethylene glycol in DMSO- $d_{6}$.

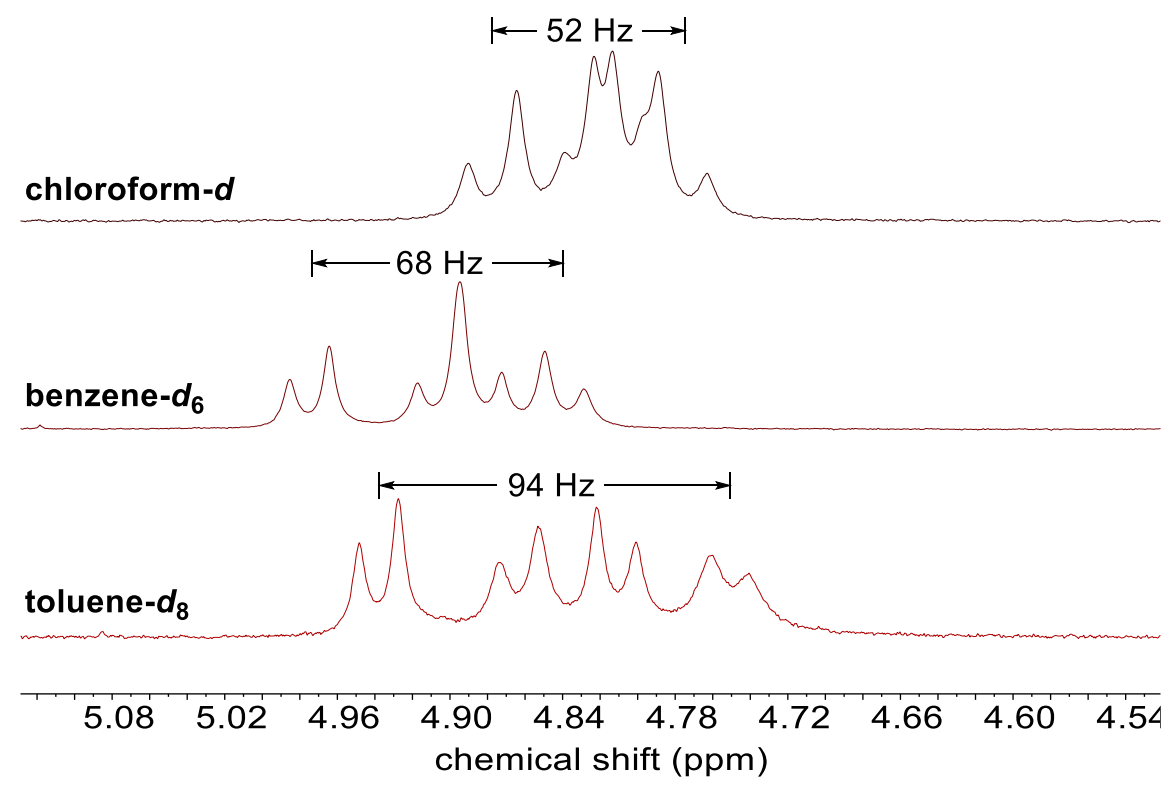

Figure S16. Partial ${ }^{1} \mathrm{H}$ NMR spectra $\left(500 \mathrm{MHz}\right.$, various solvents, $\left.[\mathrm{c}]=3 \mathrm{mM}, 25{ }^{\circ} \mathrm{C}\right)$ of selenophene-bridged helicene 1-Se. 


\section{Selenophene-bridged expanded helicene (1-Se)}

The two sets of protons $\mathrm{H}_{a} / \mathrm{H}_{\mathrm{a}^{\prime}}$ and $\mathrm{H}_{\mathrm{b}} / \mathrm{H}_{\mathrm{b}^{\prime}}$ (Scheme $\mathrm{S} 1$ ) are each diastereotopic pairs in the slow exchange regime. The enantiomerization barrier depicted above the equilibrium arrows was measured by dynamic ${ }^{1} \mathrm{H}$ NMR spectroscopy (Figure S17), as described below.

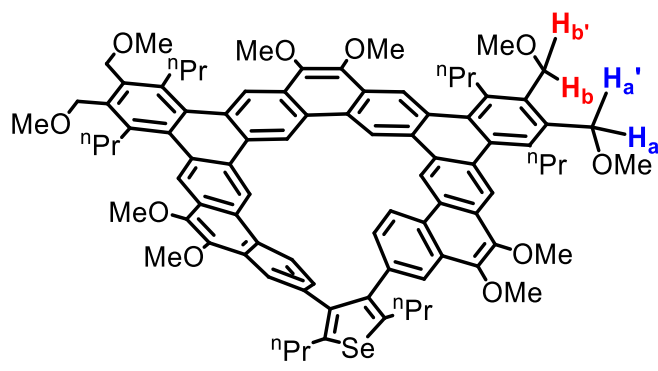

(M)-1-Se

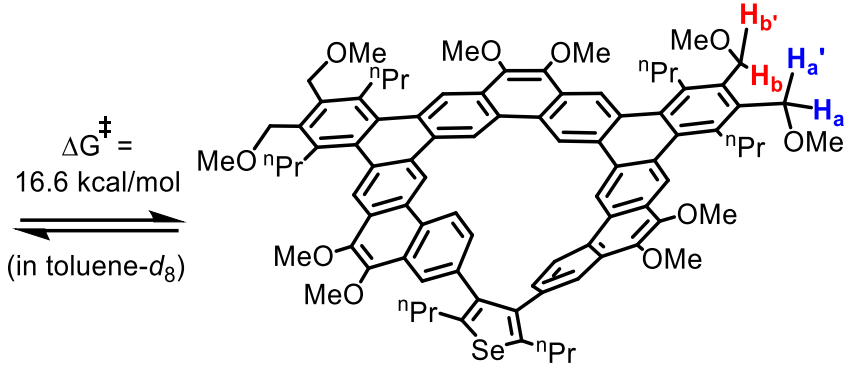

(P)-1-Se

Scheme S1. Enantiomerization of 1-Se.

At the temperature of coalescence $\left(T_{c}=338 \pm 5 \mathrm{~K}\right)$ for the two diastereotopic methylene doublets $\left(\mathrm{H}_{\mathrm{a}}\right.$ and $\mathrm{H}_{\mathrm{a}^{\prime}}$, Scheme $\left.\mathrm{S} 1\right)$, the rate of enantiomerization $\left(k_{e}=135 \pm 11 \mathrm{~s}^{-1}\right)$ was estimated using equation 1, where $\Delta v$ is the chemical shift difference of $\mathrm{H}_{\mathrm{a}}$ and $\mathrm{H}_{\mathrm{a}}$ and $J$ is the coupling constant (both in $\mathrm{Hz}$ ) of these two resonances:

$k_{e}=2.22 \sqrt{\left(\Delta v^{2}+6 J^{2}\right)}$

The Eyring equation (eq 2) was then used to calculate the enantiomerization free energy barrier $\left(\Delta \mathrm{G}^{\ddagger}{ }_{338 \mathrm{~K}}=16.6 \pm 0.3 \mathrm{kcal} / \mathrm{mol}\right)$ for 1 -Se. The major source of error in $\Delta \mathrm{G}^{\ddagger}$ is the uncertainty in measurement of $T_{c}$ due to chemical shift overlap.

$\Delta G_{T_{c}}^{\ddagger}=0.001987 * T_{c}\left[23.76+\operatorname{Ln}\left(\frac{T_{c}}{k_{e}}\right)\right]$

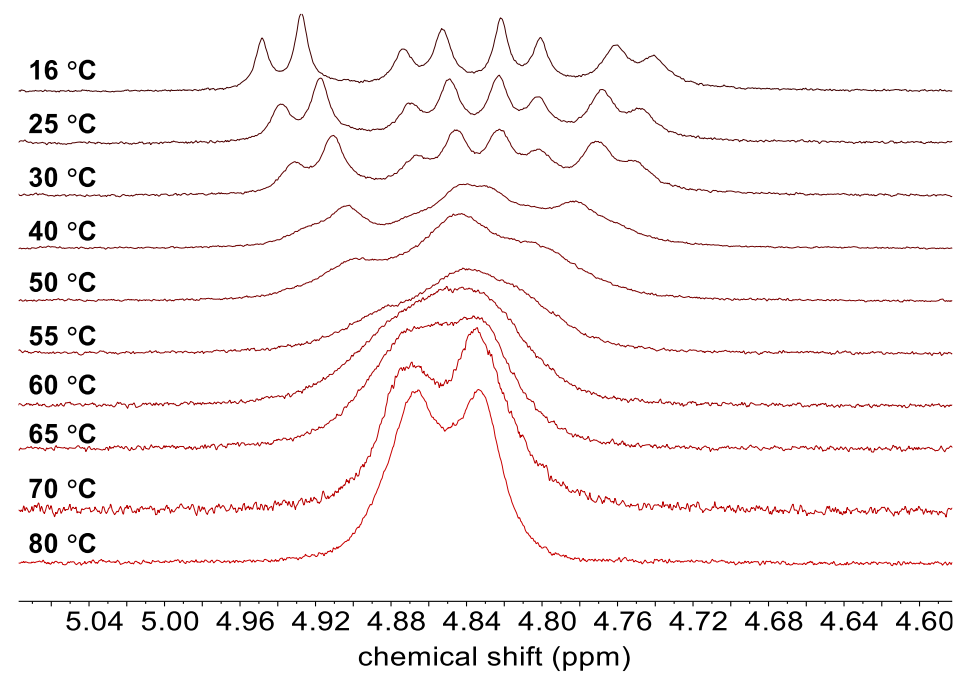

Figure S17. Variable temperature partial ${ }^{1} \mathrm{H}$ NMR spectra $\left(500 \mathrm{MHz}\right.$, toluene- $\left.d_{8}\right)$ of selenophenebridged helicene 1-Se. 


\section{Phenylene-bridged expanded helicene (1-Benz)}

For the enantiomerization process shown in Scheme S2, ${ }^{1} \mathrm{H}$ NMR spectroscopy only enabled the determination of a lower bound for $\Delta G^{\ddagger}(20.2 \mathrm{kcal} / \mathrm{mol})$. This value was estimated as follows. The two observables from the series of NMR spectra presented in Figure S18 are the maximum chemical shift difference, $\Delta V_{\max }$, and minimum coalescence temperature, $T_{\min }$. By combination of equations 1 and 2 (above), the lower bound for $\Delta G^{\ddagger}$ can be written as a function of these two observables (equation 3):

$\Delta G^{\ddagger}>0.001987 * T_{\min }\left[23.76+\operatorname{Ln}\left(\frac{T_{\min }}{2.22 \sqrt{\left(\Delta v_{\max }^{2}+6 J^{2}\right)}}\right)\right]$

Note that a maximum value of $\Delta v$ (i.e. $\Delta v_{\max }$ ) was used since these resonances appear to exhibit temperature dependence ( $\Delta v$ decreases with increasing temperature), which likely results from aggregation.

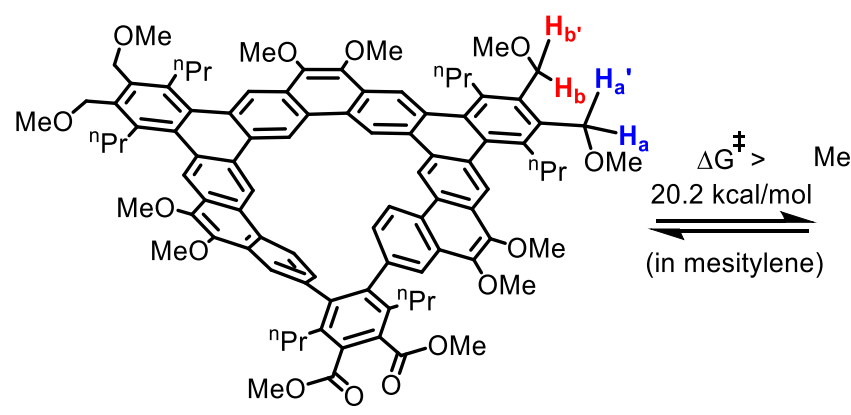

(M)-1-Benz

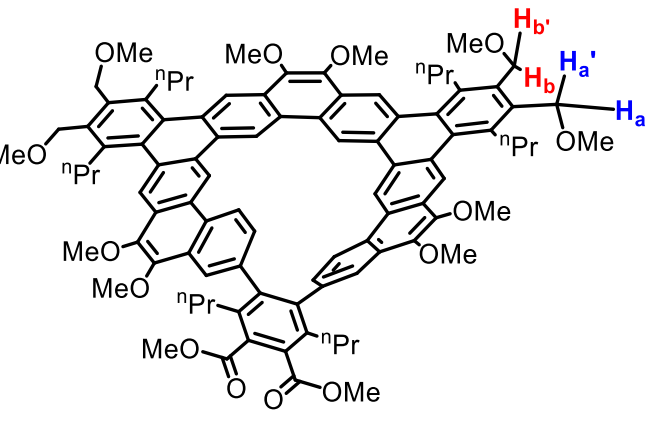

(P)-1-Benz

Scheme S2. Enantiomerization of 1-Benz. The two sets of protons $\mathrm{H}_{\mathrm{a}} / \mathrm{H}_{\mathrm{a}^{\prime}}$ and $\mathrm{H}_{\mathrm{b}} / \mathrm{H}_{\mathrm{b}^{\prime}}$ are each diastereotopic pairs in the slow exchange regime. The lower bound of the enantiomerization barrier depicted above the equilibrium arrows was measured by dynamic ${ }^{1} \mathrm{H}$ NMR spectroscopy.

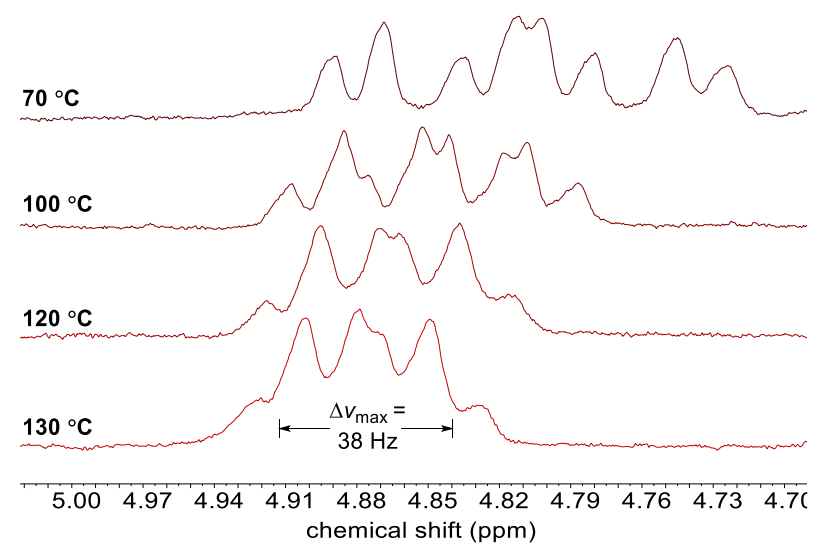

Figure S18. Variable temperature partial ${ }^{1} \mathrm{H}$ NMR spectra $\left(500 \mathrm{MHz}\right.$, mesitylene- $\left.h_{12}\right)$ of phenylene-bridged helicene 1-Benz. Chemical shifts are referenced indirectly to $\mathrm{SiMe}_{4}$ at 0.00 $\mathrm{ppm}$ via the mesitylene methyl resonance at $2.14 \mathrm{ppm}$ (the latter was determined by acquisition of a ${ }^{1} \mathrm{H}$ NMR spectrum of neat mesitylene with a $\mathrm{SiMe}_{4}$ internal standard at $22{ }^{\circ} \mathrm{C}$ ). 


\section{Figure-eight dimer (2)}

Attempts were made to measure the $\Delta \mathrm{G}^{\ddagger}$ for the enantiomerization of compound $\mathbf{2}$ (Scheme $\mathrm{S} 3$ ) in three different solvents: toluene- $d_{8}$, dichloromethane- $d_{2}$, and 1:1 toluene- $d_{8} / \mathrm{THF}$. In the fast exchange regime, $\mathrm{H}_{a} / \mathrm{H}_{\mathrm{a}^{\prime}}$ and $\mathrm{H}_{\mathrm{b}} / \mathrm{H}_{\mathrm{b}^{\prime}}$ appear as two overlapping singlets (see Figures $\mathrm{S} 19$ and $\mathrm{S} 20$ for the toluene- $d_{3}$ and dichloromethane- $d_{2}$ spectra). Upon cooling, in all solvents, these singlets broaden and then split into several different resonances. Unfortunately, inspection of the rest of the spectrum reveals that broadening and decoalescence also occurs for other, nondiastereotopic resonances (e.g. aryl protons). Thus, the splitting for $\mathrm{H}_{\mathrm{a}} / \mathrm{H}_{\mathrm{a}^{\prime}}$ and $\mathrm{H}_{\mathrm{b}} / \mathrm{H}_{\mathrm{b}^{\prime}}$ cannot be definitively attributed to the enantiomerization process.

An upper bound for $\Delta \mathrm{G}^{\ddagger}$ of $12.5 \mathrm{kcal} / \mathrm{mol}$ was determined in an analogous fashion to the lower bound for 1-Benz (above). In particular, $T_{\min }$ and $\Delta v_{\max }$ in equation 3 were replaced with $T_{\max }(248 \mathrm{~K}$ in toluene- $d_{8}$ ) and $\Delta v_{\min }$, respectively, to give equation 4 . A minimum value for $\Delta v$ (i.e. $\Delta v_{\min }=0$ ) was used since this value is unknown.

$$
\Delta G^{\ddagger}<0.001987 * T_{\max }\left[23.76+\operatorname{Ln}\left(\frac{T_{\max }}{2.22 \sqrt{\left(\Delta v_{\min }{ }^{2}+6 J^{2}\right)}}\right)\right]
$$

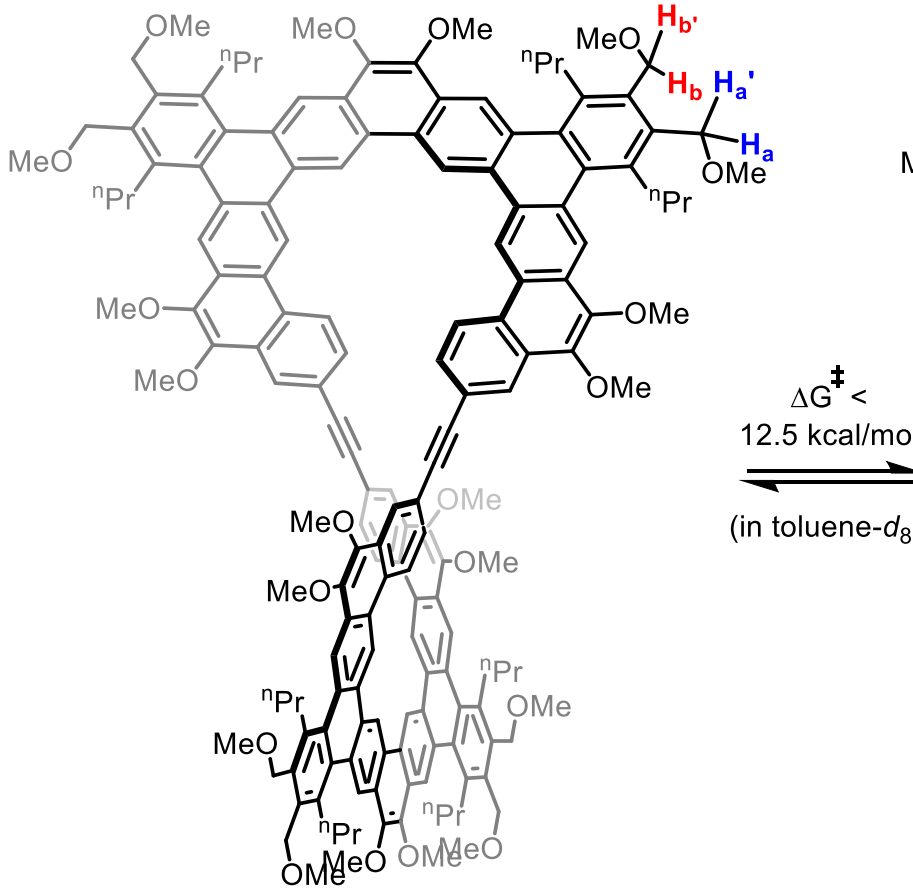

$(M, M)-\mathbf{2}$

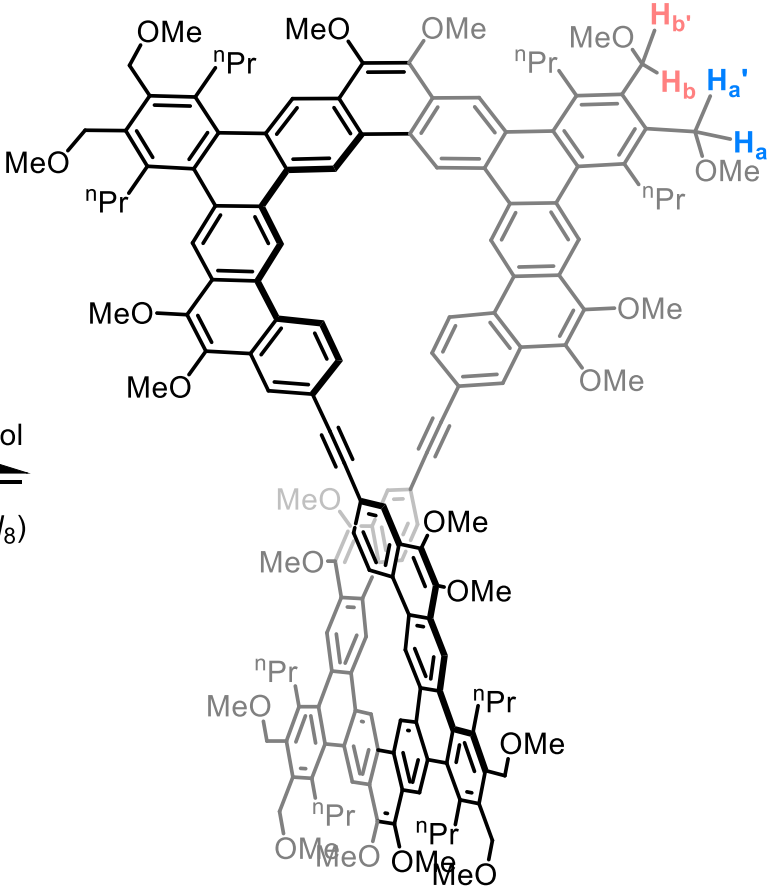

$(P, P)-\mathbf{2}$

Scheme S3. Enantiomerization of 2. The upper bound of the enantiomerization barrier depicted above the equilibrium arrows was measured by dynamic ${ }^{1} \mathrm{H}$ NMR spectroscopy. 


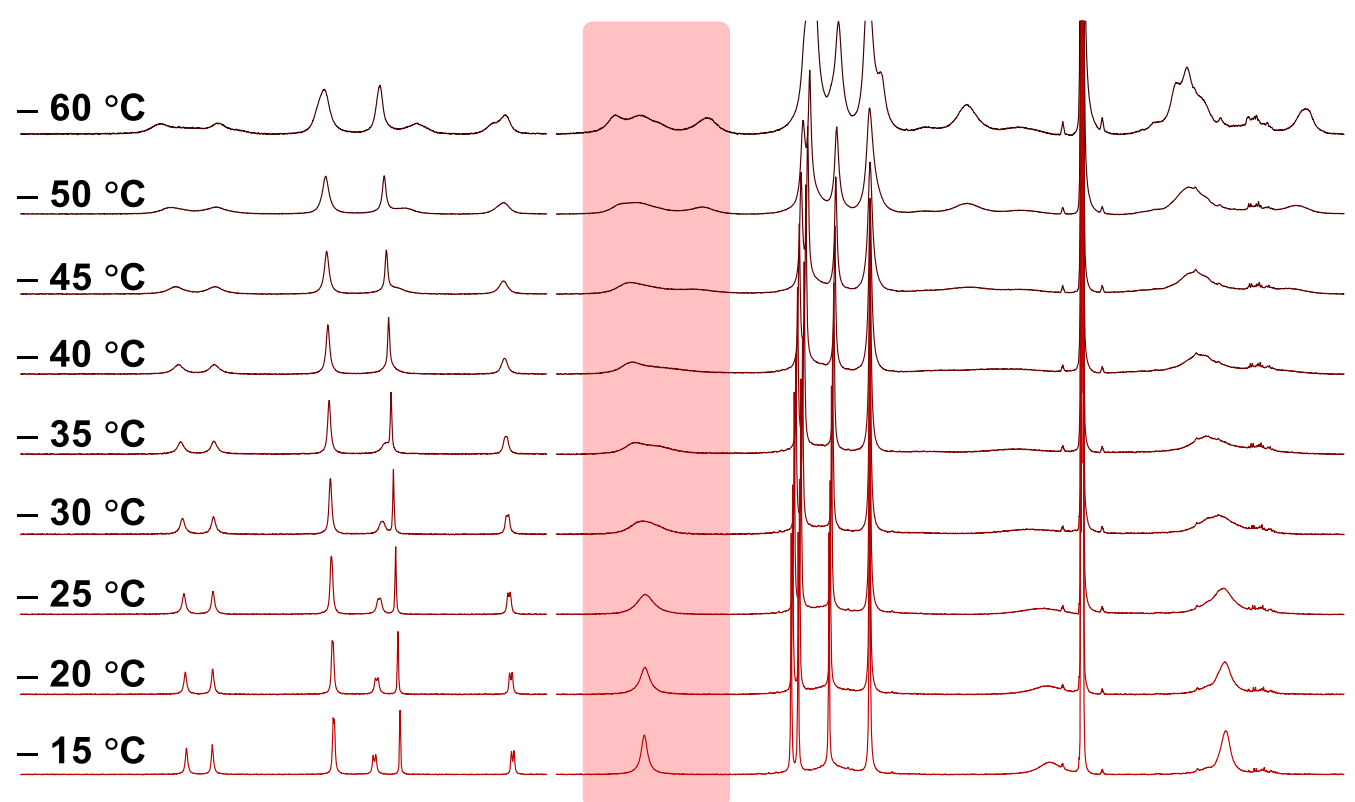

$$
\begin{aligned}
& \begin{array}{lllllllllllllllll}
10.5 & 9.5 & 8.5 & 5.0 & 4.5 & 4.0 & 3.5 & 3.0 & 2.5 & 2.0 & 1.5 & 1.0 & 0 .
\end{array} \\
& \text { chemical shift (ppm) }
\end{aligned}
$$

Figure S19. Variable temperature partial ${ }^{1} \mathrm{H}$ NMR spectra (500 MHz, toluene- $d_{8}$ ) of figure-eight dimer 2. The highlighted region corresponds to $\mathrm{H}_{a} / \mathrm{H}_{\mathrm{a}}$, and $\mathrm{H}_{\mathrm{b}} / \mathrm{H}_{\mathrm{b}}$, in Scheme S3.

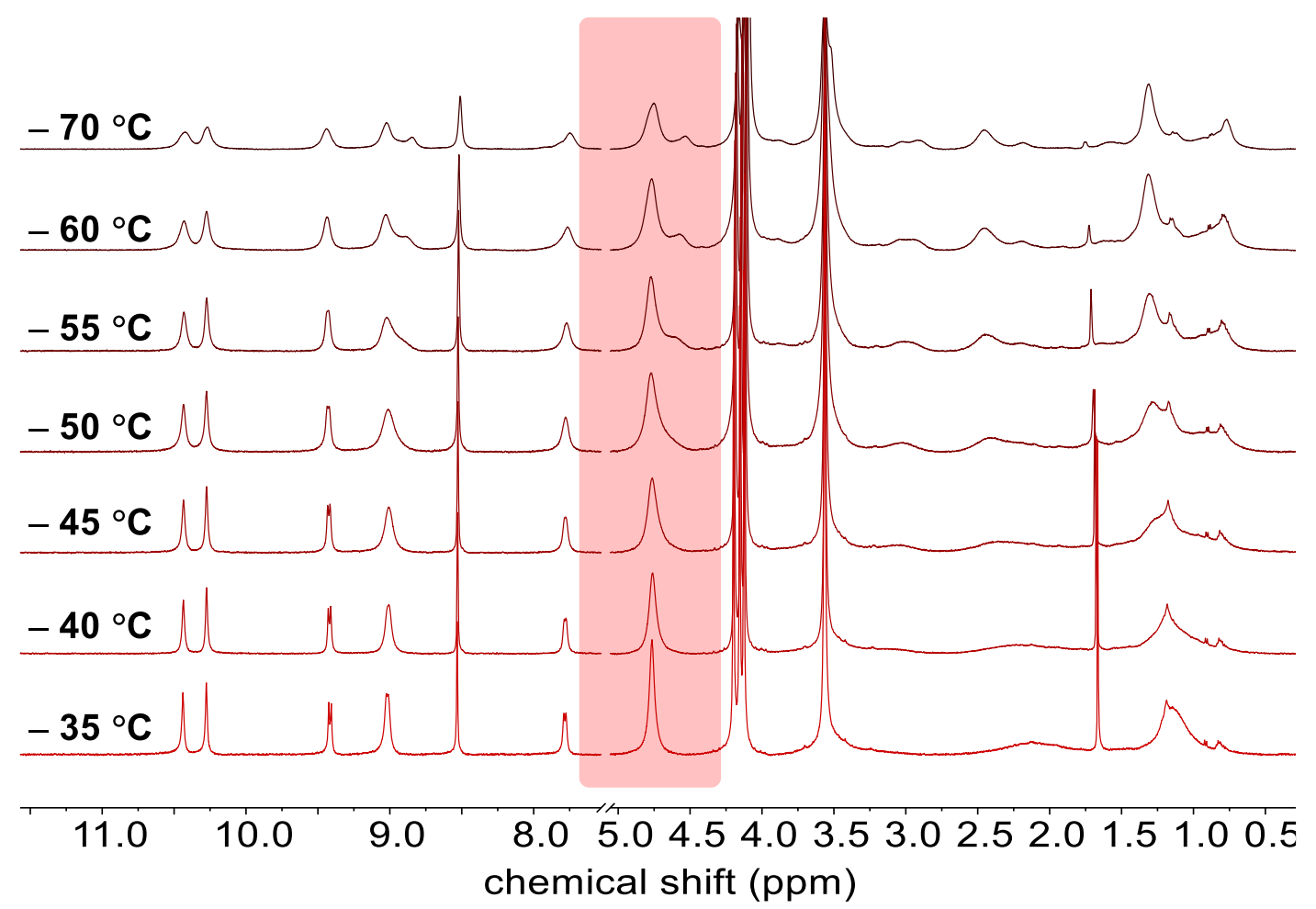

Figure S20. Variable temperature partial ${ }^{1} \mathrm{H}$ NMR spectra $\left(500 \mathrm{MHz}\right.$, dichloromethane- $\left.d_{2}\right)$ of figure-eight dimer 2. The highlighted region corresponds to $\mathrm{H}_{a} / \mathrm{H}_{\mathrm{a}}$ and $\mathrm{H}_{\mathrm{b}} / \mathrm{H}_{\mathrm{b}}$, in Scheme S3. 


\section{Alkynylated expanded helicene (3)}

Attempts were made to measure the $\Delta \mathrm{G}^{\ddagger}$ for the enantiomerization of compound 3 (Scheme S4) in three different solvents: toluene- $d_{8}$, dichloromethane- $d_{2}$, and $1: 1$ toluene- $d_{8} / \mathrm{THF}$. In the fast exchange regime, $\mathrm{H}_{\mathrm{a}} / \mathrm{H}_{\mathrm{a}}$ and $\mathrm{H}_{\mathrm{b}} / \mathrm{H}_{\mathrm{b}}$, appear as two overlapping singlets (see Figures S21 and S22 for the toluene- $d_{8}$ and dichloromethane- $d_{2}$ spectra). Upon cooling, in all solvents, these singlets broaden and then split into several different resonances. Unfortunately, inspection of the rest of the spectrum reveals that broadening and decoalescence also occurs for other, nondiastereotopic resonances (e.g. aryl protons). Thus, the splitting for $\mathrm{H}_{\mathrm{a}} / \mathrm{H}_{\mathrm{a}^{\prime}}$ and $\mathrm{H}_{\mathrm{b}} / \mathrm{H}_{\mathrm{b}}$, cannot be definitively attributed to the enantiomerization process.

An upper bound for $\Delta \mathrm{G}^{\ddagger}$ of $11.9 \mathrm{kcal} / \mathrm{mol}$ was determined in an analogous fashion to that for 2 (see above), where $T_{\max }=238 \mathrm{~K}$ in toluene- $d_{8}$.

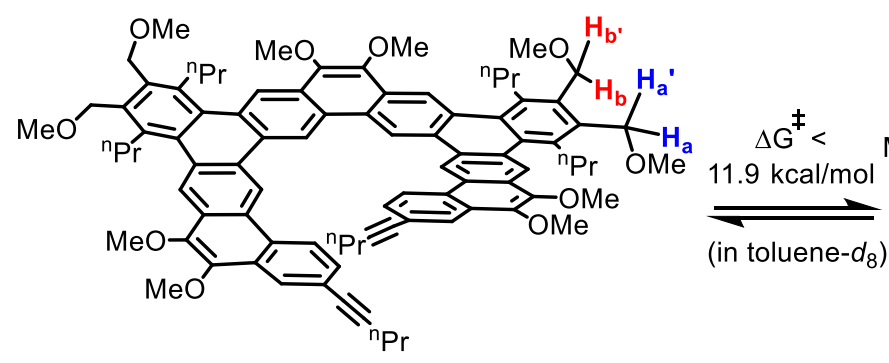

$(M)-3$

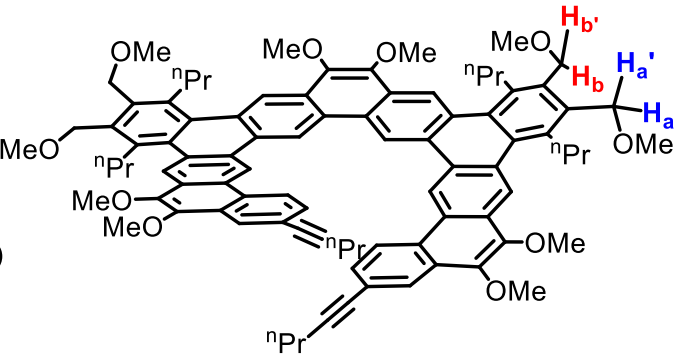

$(P)-3$

Scheme S4. Enantiomerization of 3. The upper bound of the enantiomerization barrier depicted above the equilibrium arrows was measured by dynamic ${ }^{1} \mathrm{H}$ NMR spectroscopy.

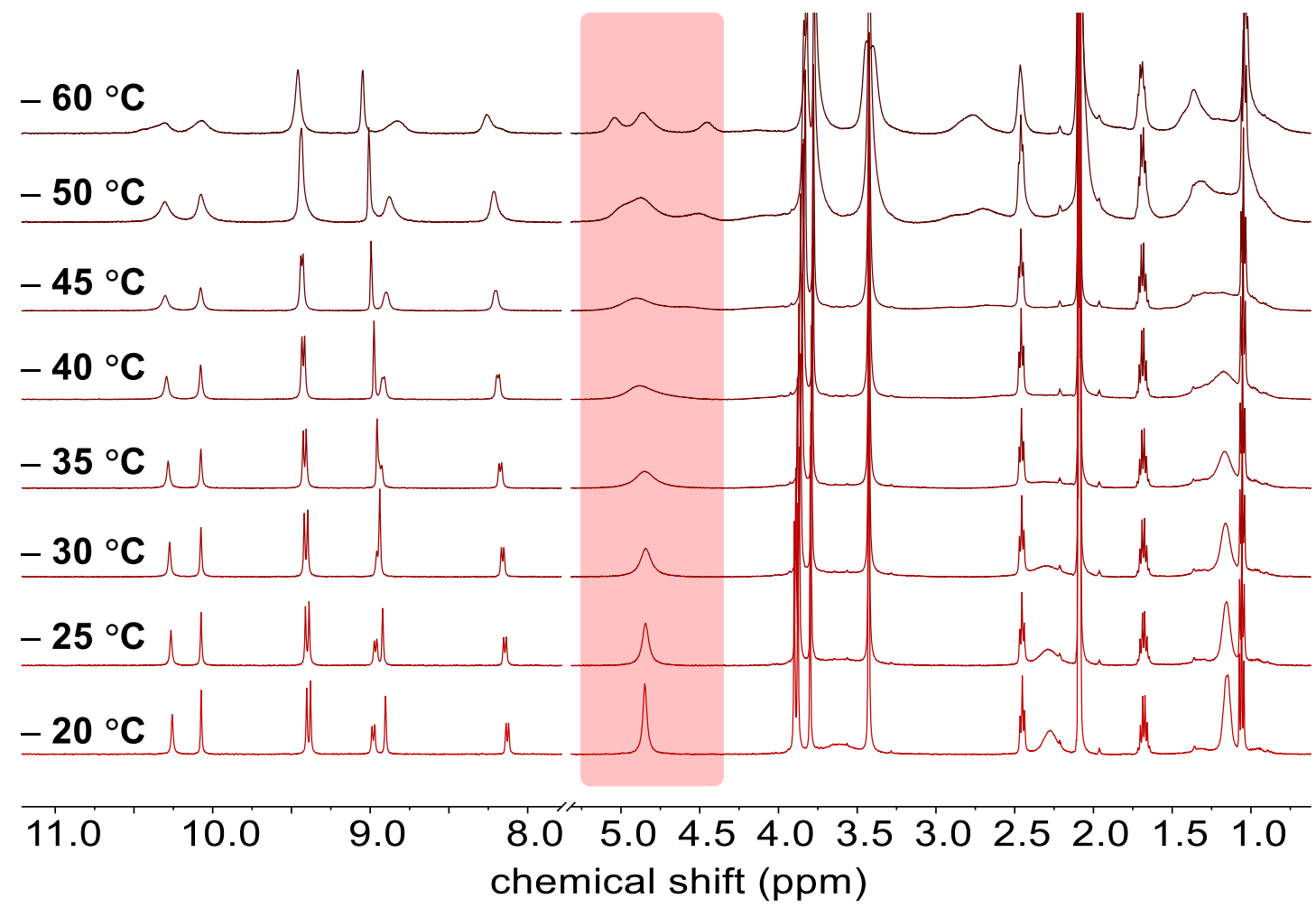

Figure S21. Variable temperature partial ${ }^{1} \mathrm{H}$ NMR spectra $\left(500 \mathrm{MHz}\right.$, toluene- $\left.d_{8}\right)$ of helicene 3. The highlighted region corresponds to $\mathrm{H}_{\mathrm{a}} / \mathrm{H}_{\mathrm{a}^{\prime}}$ and $\mathrm{H}_{\mathrm{b}} / \mathrm{H}_{\mathrm{b}}$, in Scheme $\mathrm{S} 4$. 


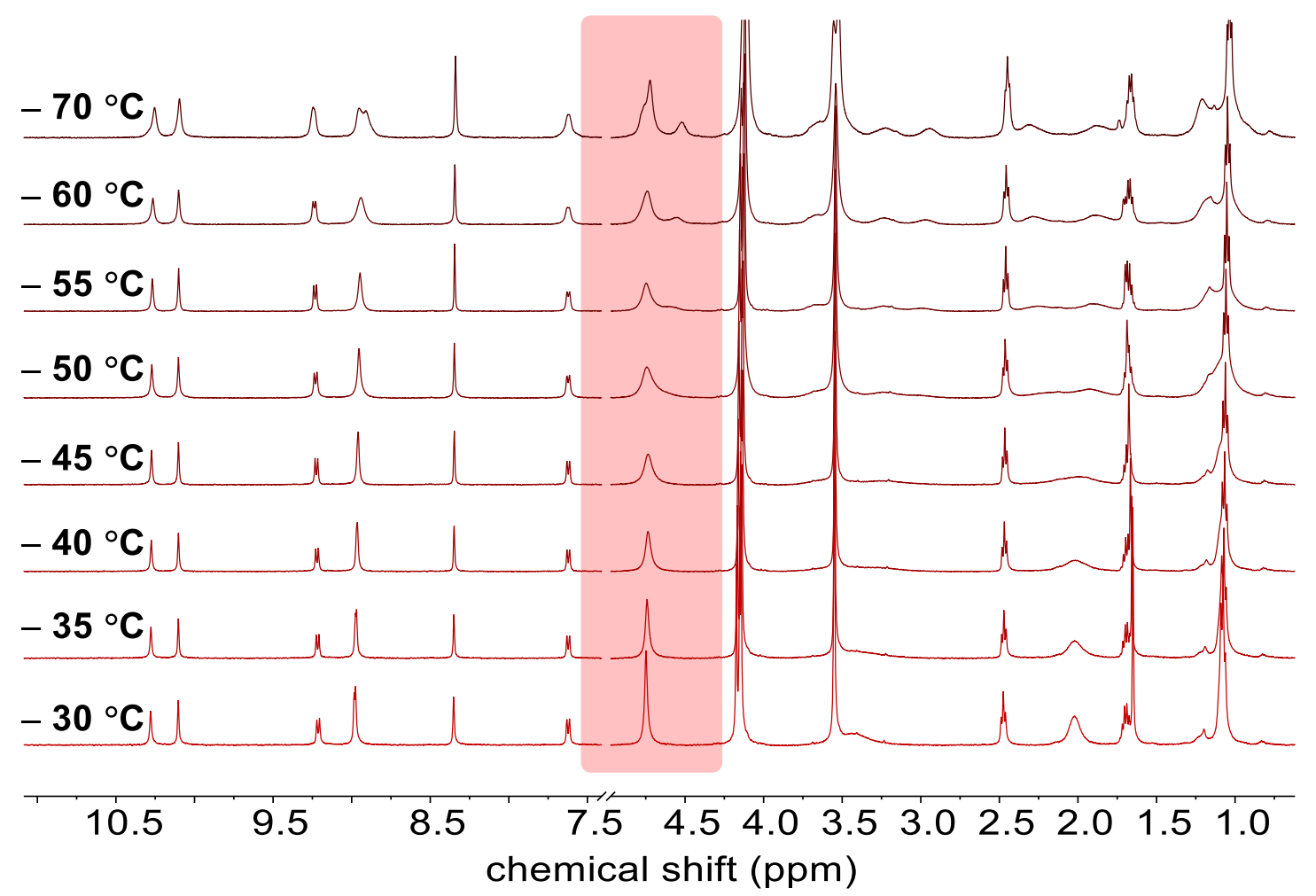

Figure S22. Variable temperature partial ${ }^{1} \mathrm{H}$ NMR spectra $\left(500 \mathrm{MHz}\right.$, dichloromethane- $\left.d_{2}\right)$ of helicene 3. The highlighted region corresponds to $\mathrm{H}_{a} / \mathrm{H}_{\mathrm{a}^{\prime}}$ and $\mathrm{H}_{\mathrm{b}} / \mathrm{H}_{\mathrm{b}}$ ' in Scheme S4. 


\section{Determination of the enantiomerization barrier of 1-Benz via dynamic circular dichroism (CD) spectroscopy}

Since the enantiomerization barrier for 1-Benz is too high to measure using dynamic ${ }^{1} \mathrm{H}$ NMR spectroscopy (see above), it was measured by dynamic CD spectroscopy. ${ }^{14}$ Table $\mathrm{S} 1$ summarizes the results of these studies in two different solvents (1,2-dichlorobenzene and toluene). The data were obtained by separation of the enantiomers by chiral HPLC (Figure S23) and following the decay in CD over time at $25^{\circ} \mathrm{C}$ (Figures S25 and S26). The decay in CD was then used to measure the first order rate constant for racemization (Figures S27 and S28).

\begin{tabular}{|l|l|l|}
\hline & toluene & 1,2 -dichlorobenzene \\
\hline Temperature $(\mathrm{K})$ & $298 \pm 1$ & $298 \pm 1$ \\
\hline $\begin{array}{l}\text { First-order rate constant } \\
\text { for racemization at 298 K } \\
\left(\mathrm{s}^{-1}\right)\end{array}$ & $(7.24 \pm 0.14) \times 10^{-4}$ & $(6.44 \pm 0.29) \times 10^{-4}$ \\
\hline $\begin{array}{l}\text { Racemization half-life at } \\
298 \mathrm{~K}(\mathrm{~s})\end{array}$ & 957 & $1.08 \times 10^{3}$ \\
\hline $\begin{array}{l}\text { Racemization half-life at } \\
298 \mathrm{~K}(\mathrm{~min})\end{array}$ & 16.0 & 17.9 \\
\hline $\begin{array}{l}\text { Enantiomerization free } \\
\text { energy barrier at 298 K, } \\
\Delta G_{298 \mathrm{~K}}^{\ddagger}(\mathrm{kcal} / \mathrm{mol})\end{array}$ & $22.1 \pm 0.1$ & $22.2 \pm 0.1$ \\
\hline
\end{tabular}

Table S1. Summary of racemization and enantiomerization data for 1-Benz in 1,2dichlorobenzene and toluene, which were calculated from the data shown in Figures S25-S28.

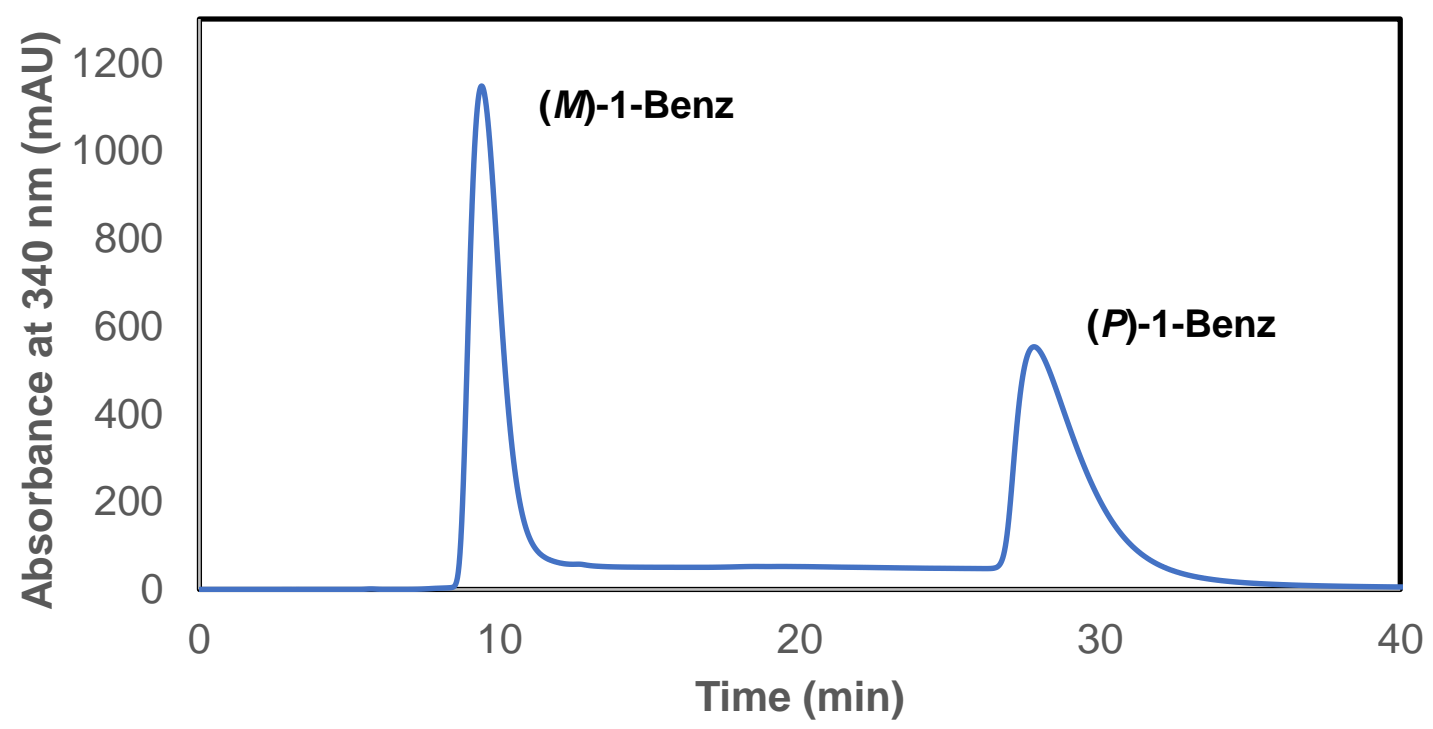

Figure S23. Resolution of the enantiomers of 1-Benz by chiral analytical HPLC. The enantiomers were resolved from $\leq 1.0 \mathrm{mg}$ of racemic mixture dissolved in $2 \mathrm{~mL}$ of $50 \%$ toluene/hexanes. This solution was injected in $\leq 200 \mu \mathrm{L}$ aliquots onto a CHIRALPAK ${ }^{\circledR}$ IA-3 column $(4.6 \mathrm{~mm}$ I.D. $\times 250$ $\mathrm{mm}, 3 \mu \mathrm{m}$ ), with $50 \%$ toluene/hexanes eluent flowing at $1 \mathrm{~mL} / \mathrm{min}$ at $25^{\circ} \mathrm{C}$. The $M$ configuration 
for molecules in the sample that eluted at $\sim 10$ min was determined by comparison of the experimental CD spectrum with one that was computed for the M enantiomer (Figure S24).

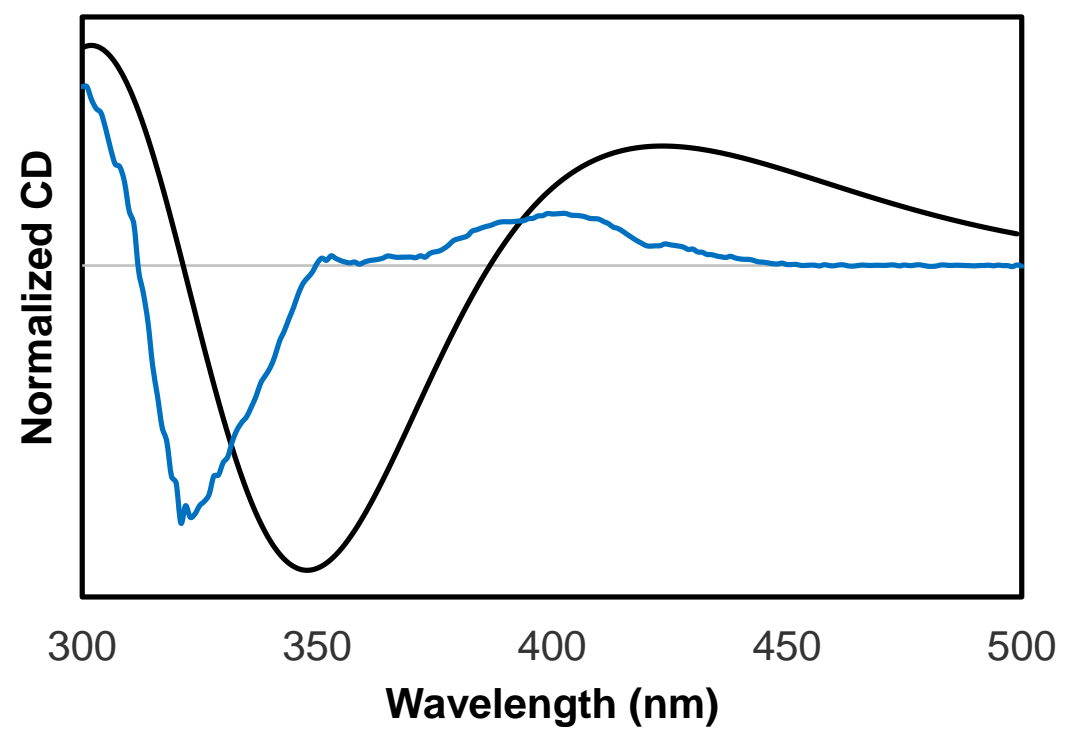

Figure S24. TD-DFT-computed CD spectrum for ( $M$ )-1-Benz (black) overlaid on the experimental spectrum for enantioenriched 1-Benz (blue; reproduced from Figure S25). The lowest 50 excited electronic states were computed using TD-B3LYP-D3/6-31G(d). A polarizable continuum model IEF-PCM for solvation by toluene was used for all excited state computations. The CD spectrum was generated using Gauss View 6.0.16 based on rotary strengths resulting from the TD-DFT calculation.

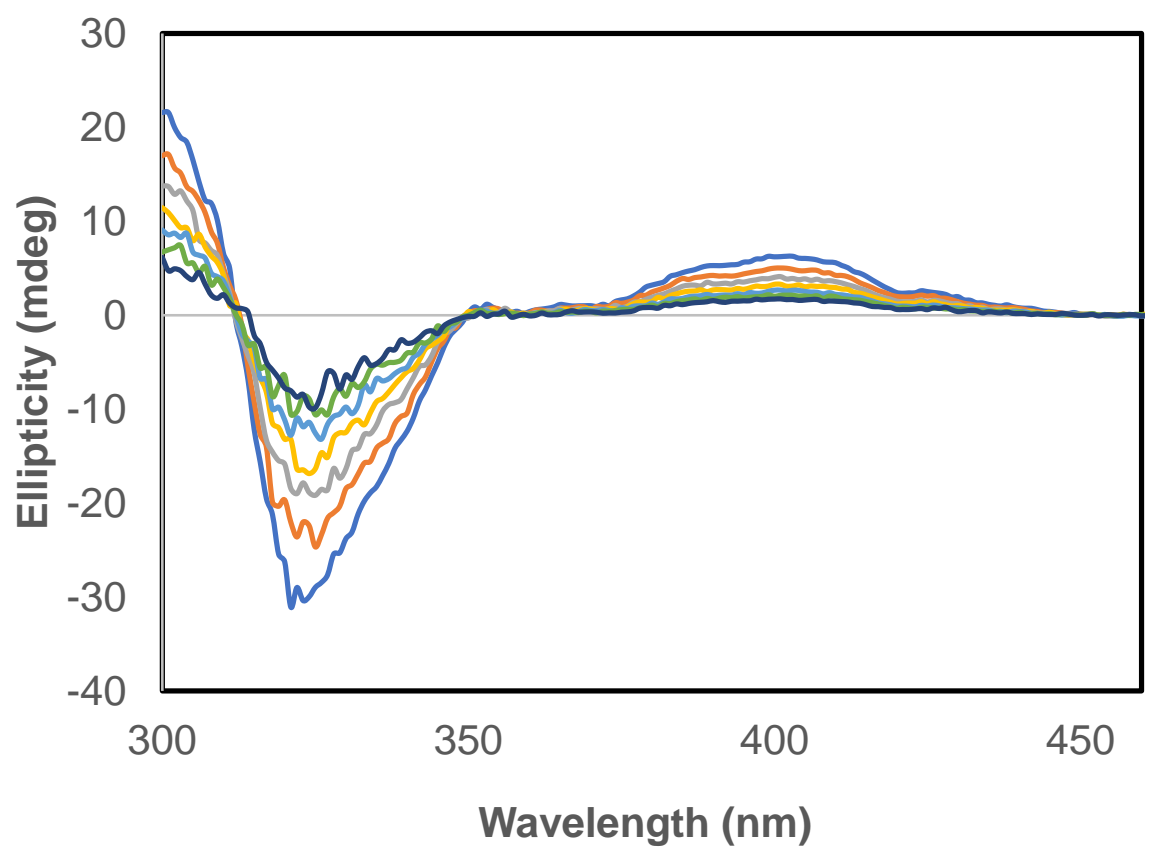

Figure S25. Time-evolved CD spectra for enantioenriched 1-Benz at $25^{\circ} \mathrm{C}$ in toluene. The spectra were recorded in 5 min increments. 


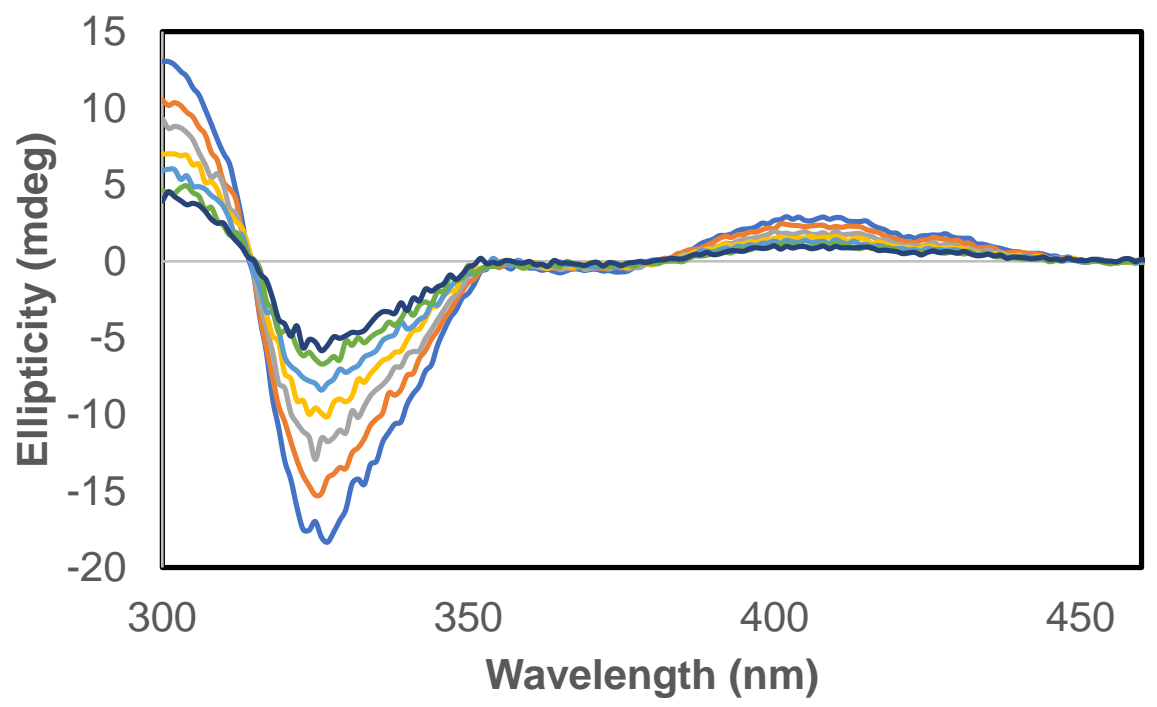

Figure S26. Time-evolved $C D$ spectra for enantioenriched 1-Benz at $25{ }^{\circ} \mathrm{C}$ in $1,2-$ dichlorobenzene. The spectra were recorded in 5 min increments.

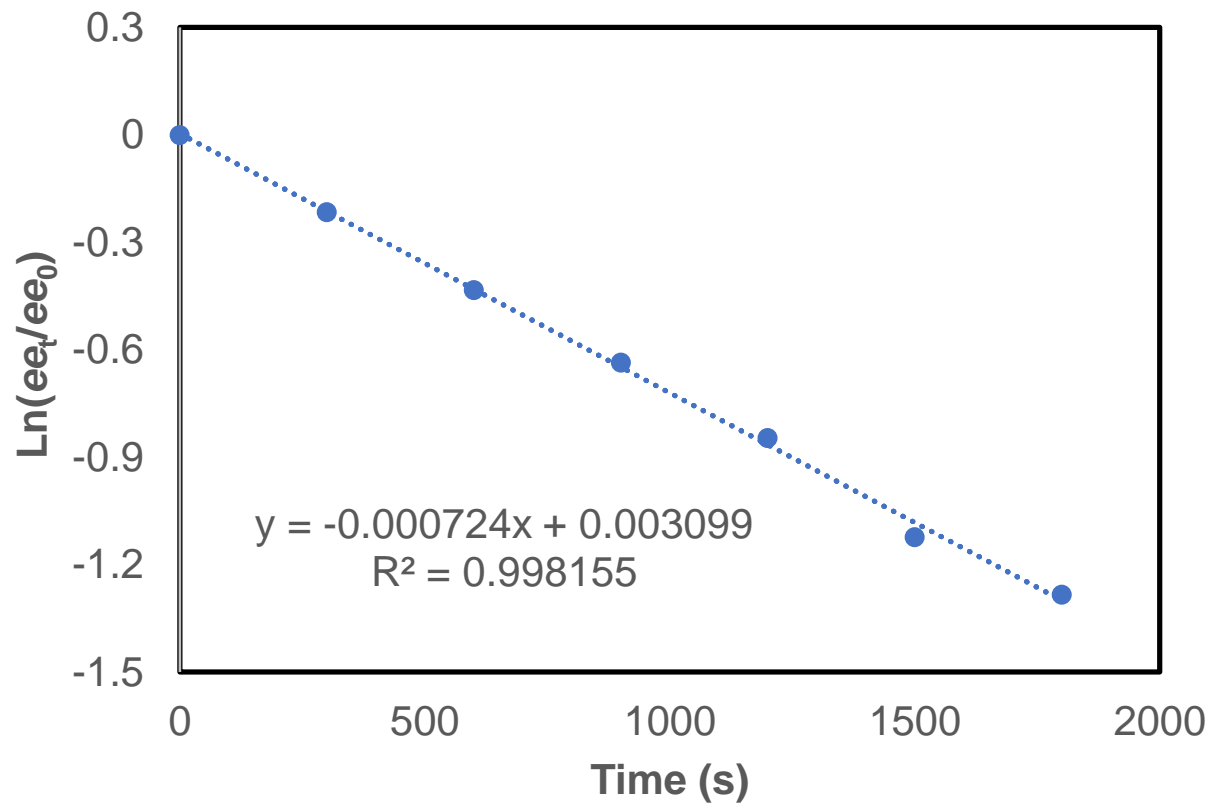

Figure S27. Plot of $\operatorname{Ln}\left(e e_{t} / e e_{0}\right)$ vs. time for $1-B e n z$ at $25{ }^{\circ} \mathrm{C}$ in toluene. The ratio $e e_{/} / e e_{0}$ was determined from the CD recorded at $400 \mathrm{~nm}$ in the time-evolved spectra shown in Figure S25. 


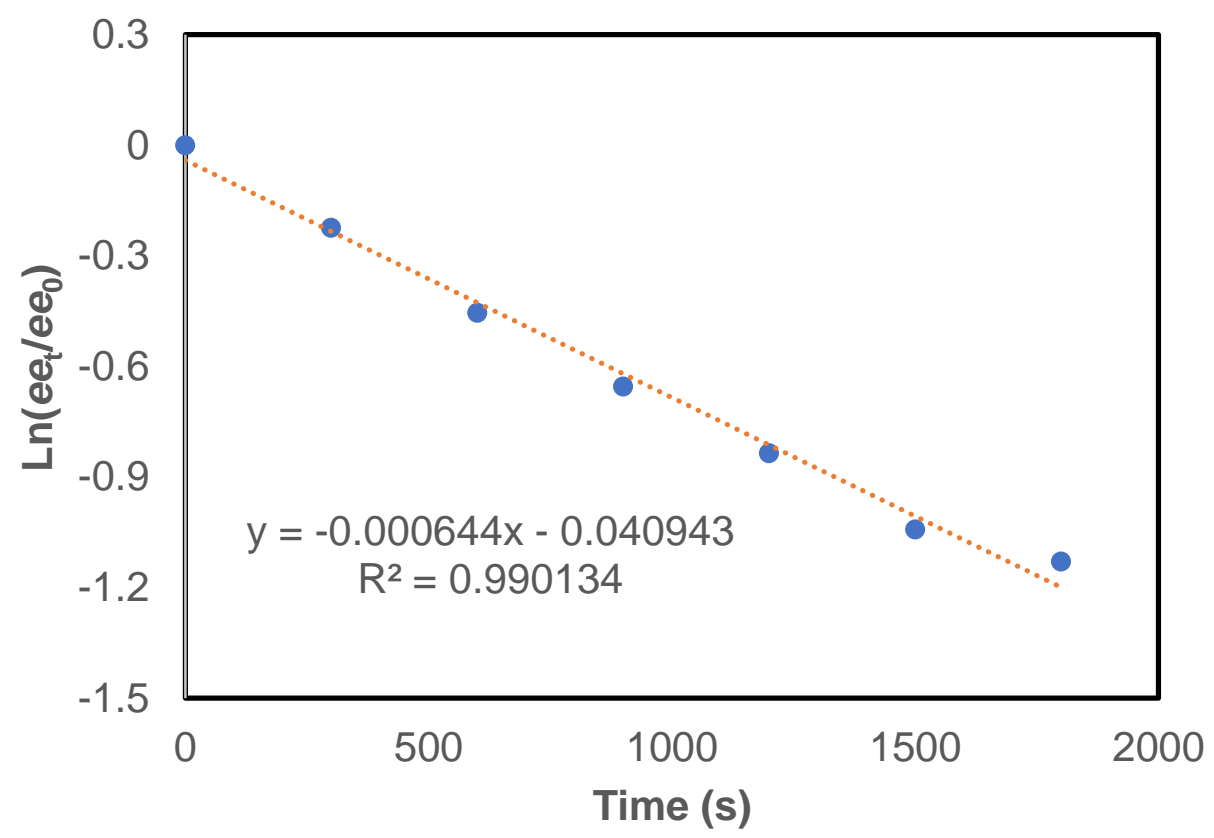

Figure S28. Plot of $\operatorname{Ln}\left(e e_{t} / e_{0}\right)$ vs. time for 1-Benz at $25^{\circ} \mathrm{C}$ in 1,2-dichlorobenzene. The ratio $e e_{t} / e e_{0}$ was determined from the CD recorded at $323 \mathrm{~nm}$ in the time-evolved spectra shown in Figure S26. 


\section{Photophysical characterization}

UV/Vis spectra were recorded on a Varian Cary 300 Bio UV-Visible spectrophotometer. Emission spectra were recorded on a Varian Cary Eclipse Spectrometer. All compounds were purified by preparatory thin layer chromatography $\left(\mathrm{CH}_{2} \mathrm{Cl}_{2} / \mathrm{EtOAc}\right.$ solvent system) prior to analysis to ensure high purity. The UV/Vis spectra that are shown represent the average of two measurements on separate samples to ensure accuracy of extinction coefficients. For sample preparation, each analyte was weighed on an analytical balance and dissolved in the appropriate amount of $\mathrm{CH}_{2} \mathrm{Cl}_{2}$ using a volumetric flask. A single dilution experiment (10x) was performed for each compound and the UV/vis spectra (plotted by molar absorptivity) for all compounds were unchanged by this dilution. Emission spectra for all compounds were independent of excitation wavelength.

\begin{tabular}{|l|l|l|l|l|}
\hline Compound & $\begin{array}{l}\text { Absorption } \\
\text { Onset, } \lambda_{\text {onset }} \\
(\mathrm{nm})^{\mathrm{a}, \mathrm{b}}\end{array}$ & $\begin{array}{l}\text { Absorption } \\
\text { Maximum, } \\
\lambda_{\max }(\mathrm{nm})^{\mathrm{a}}\end{array}$ & $\begin{array}{l}\text { Emission } \\
\text { Maximum, } \\
\lambda_{\max }(\mathrm{nm})^{\mathrm{a}}\end{array}$ & $\begin{array}{l}\text { Photophysical } \\
\text { HOMO-LUMO } \\
\text { gap (eV) })^{\mathrm{a}, \mathrm{c}}\end{array}$ \\
\hline $1-$ Benz & 423 & 319 & 452 & 2.93 \\
\hline $1-\mathrm{Se}$ & 424 & 322 & 451 & 2.92 \\
\hline 2 & 424 & 334 & 449 & 2.92 \\
\hline 3 & 420 & 322 & 448 & 2.95 \\
\hline
\end{tabular}

Table S2. Summary of relevant photophysical properties. (a) Solvent $=\mathrm{CH}_{2} \mathrm{Cl}_{2}$; (b) Defined as the point at which the absorption and emission spectra intersect; ${ }^{15}$ (c) Estimated from the absorption onset $\left(\lambda_{\text {onset }}\right): E_{g}=1240 / \lambda_{\text {onset }}$

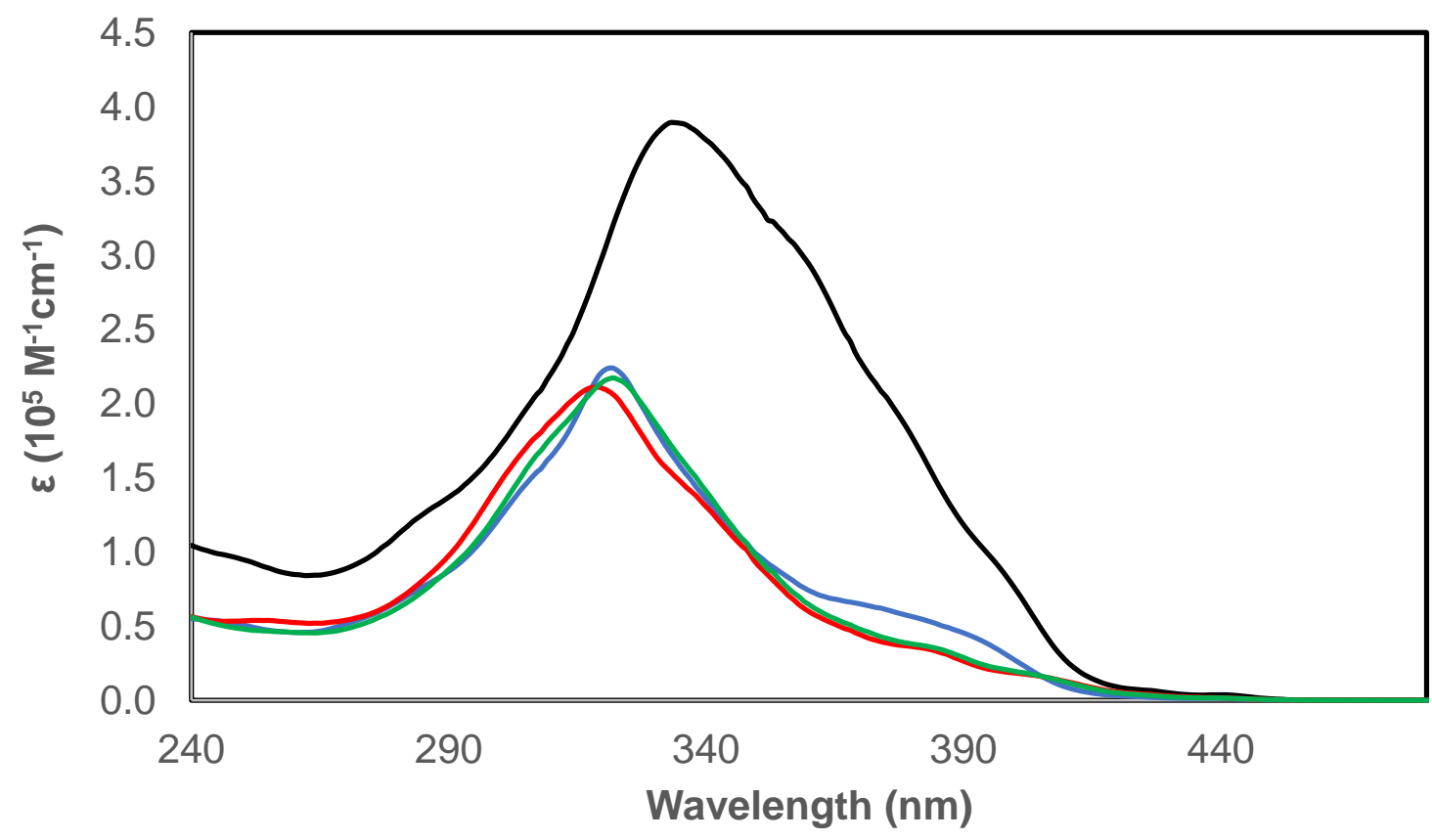

Figure S29. UV/Vis absorption spectra for helicene 3 (blue) and macrocyclic derivatives 1-Benz (red), 1-Se (green), and 2 (black) in $\mathrm{CH}_{2} \mathrm{Cl}_{2}$ solvent ( $\varepsilon=$ molar absorptivity). 


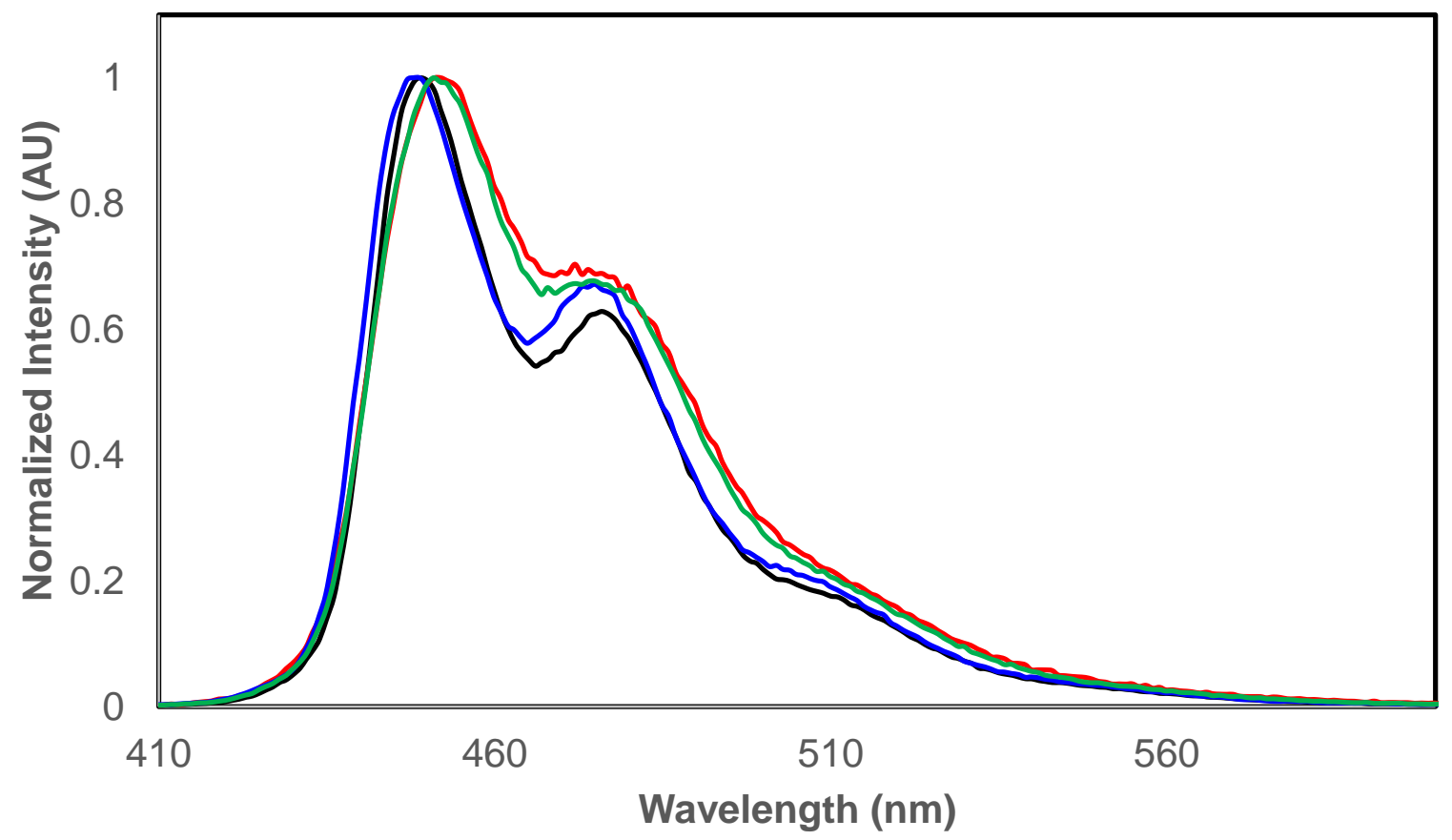

Figure S30. Normalized emission spectra for precursor helicene 3 (blue) and macrocyclic derivatives 1-Benz (red), 1-Se (green), and 2 (black) in $\mathrm{CH}_{2} \mathrm{Cl}_{2}$ solvent. 


\section{X-ray crystallography}

\section{Experimental Details}

X-ray diffraction data were collected at beamline 12.2.1 of the Advanced Light Source at Lawrence Berkeley National Laboratory. Frames were collected on a PHOTON 100 CMOS detector using radiation with a wavelength of $0.7288 \AA$ selected by a $\mathrm{Si}(111)$ monochromator and focused to $200 \mu \mathrm{m}^{2}$ with a toroidal mirror. Data collection, integration, scaling, and space group determination were performed with Bruker APEX3 (v. 2016.5-0) software. Structures were solved by SHELXT-2014 and refined with SHELXL-2014, with refinement of $F^{2}$ on all data by full-matrix least squares. ${ }^{16-19}$ The $3 \mathrm{D}$ molecular structure figures were produced in Mercury $3.7 .{ }^{20}$

The single crystals that were used for this study were grown as follows. In a $6 \mathrm{~mL}$ vial, compound $2(3 \mathrm{mg})$ was dissolved in $\mathrm{CH}_{2} \mathrm{Cl}_{2}(0.8 \mathrm{~mL})$, then the solution was diluted with hexanes $(0.8 \mathrm{~mL})$. The vial was capped, and the lid was punctured with 2-3 pinholes. In a dark cabinet, the solvent was allowed evaporate to $\sim 1 / 3$ of the original volume over the course to 2-3 days, resulting in the formation of yellow, irregularly shaped crystals. Crystals of 2 were weakly diffracting, and substantially increased exposure times did not result in appreciably improved resolution. Extensive use of SIMU and ISOR restraints were required to maintain reasonable anisotropic displacement parameters for some of the atoms in the structural model. A void with a volume of $5958 \AA^{3}$ per unit cell containing 2316 e was also found, which is consistent with the presence of $\sim 48$ disordered $n$-hexane molecules per unit cell, or four $n$-hexane molecules per asymmetric unit. The electron density for these disordered solvent molecules was treated with the SQUEEZE program. ${ }^{21}$

\begin{tabular}{|c|c|}
\hline Empirical formula & $\mathrm{C}_{160} \mathrm{H}_{148} \mathrm{O}_{20}+16\left[\mathrm{C}_{6} \mathrm{H}_{14}\right]$ \\
\hline Formula weight & 3769.51 \\
\hline Temperature & 100.15 \\
\hline Crystal system & Hexagonal \\
\hline Space group & $P 6422$ \\
\hline \multirow[t]{6}{*}{ Unit cell dimensions } & $a=31.7473(18) \AA$ \\
\hline & $b=31.7473(18) \AA$ \\
\hline & $C=16.1631(10 \AA$ \\
\hline & $\alpha=90^{\circ}$ \\
\hline & $\beta=90^{\circ}$ \\
\hline & $Y=120^{\circ}$ \\
\hline Volume & $14108.1(18) \AA^{3}$ \\
\hline Z & 3 \\
\hline Density (calculated) & $1.331 \mathrm{~g} / \mathrm{cm}^{3}$ \\
\hline$\mu$ & $0.085 \mathrm{~mm}^{-1}$ \\
\hline$F(000)$ & 6204.0 \\
\hline Crystal size & $0.15 \times 0.15 \times 0.15 \mathrm{~mm}^{3}$ \\
\hline Radiation & Synchrotron $(\lambda=0.7288 \AA)$ \\
\hline \multicolumn{2}{|c|}{$2 \Theta$ range for data collection 1.518 to $45.412^{\circ}$} \\
\hline Index ranges & $-33 \leq h \leq 33,-33 \leq k \leq 33,-17 \leq 1 \leq 17$ \\
\hline Reflections collected & 128453 \\
\hline Independent reflections & $5859\left[R_{\text {int }}=0.1259, R_{\text {sigma }}=0.0408\right]$ \\
\hline
\end{tabular}




$\begin{array}{ll}\text { Data/restraints/parameters } & 5859 / 102 / 423 \\ \text { Goodness-of-fit on } \mathrm{F}^{2} & 1.282 \\ \text { Final } \mathrm{R} \text { indexes [l>=2 } \sigma(\mathrm{I})] & \mathrm{R}_{1}=0.1084, \mathrm{wR}_{2}=0.2776 \\ \text { Final } \mathrm{R} \text { indexes [all data] } & \mathrm{R}_{1}=0.1296, \mathrm{wR}_{2}=0.3056 \\ \text { Largest diff. peak/hole } & 0.57 /-0.47 \mathrm{e}^{-3} \\ \text { Flack parameter } & 0(3)\end{array}$

Table S3. Crystal data and structure refinement for figure-eight dimer 2.

\section{Comparison of X-ray and gas-phase (DFT-calculated) structures}

The lowest-energy gas-phase geometry of $\mathbf{2}$ (as predicted by DFT) is notably different from that observed in the solid-state (Figure S31). The most important differences involve the spatial relationship between the two constituent helicene units. This can be described in part by the lower symmetry for the DFT-calculated structure (pseudo- $C_{2}$ vs. $D_{2}$ in the solid-state), which is due to the "bend" shown in Figure S31a. Another notable descriptor is the angle between the leastsquares planes bisecting the helicene units ( $40^{\circ}$ in the gas-phase vs. $30^{\circ}$ in the solid-state), which provides a measure of the "twist" of 2 (Figure S31b-c). Interestingly, DFT calculations suggest an energy cost of only $0.6 \mathrm{kcal} / \mathrm{mol}$ for these conformational differences, which was estimated by a comparison of the gas-phase energies of the $D_{2}$ and pseudo- $C_{2}$ structures.

(a)

(c)
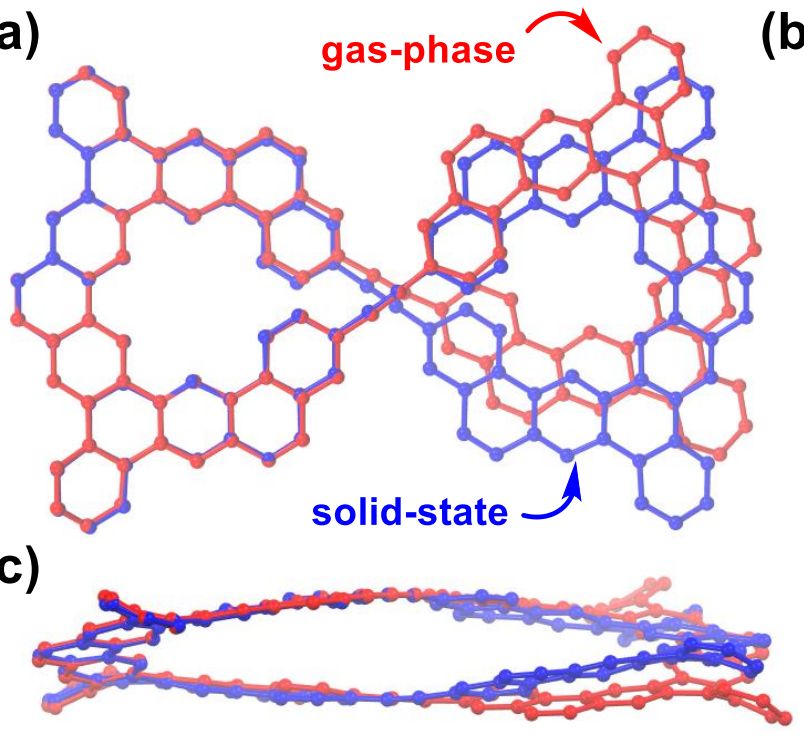

(b)

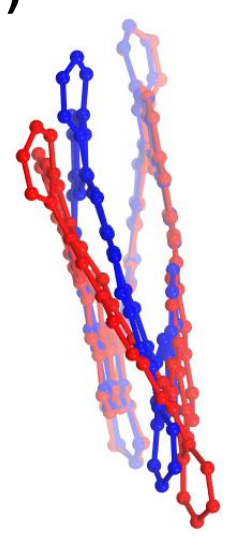

Figure S31. Comparison of the solid-state (blue; $D_{2}$ symmetry) and lowest energy gas-phase (red; pseudo- $C_{2}$ symmetry) molecular structures of 2 . The former is a reproduction of that shown in the main text (Figure 2) and the latter was calculated by DFT (B3LYP-D3/6-31G(d)). Side chains are truncated for clarity. (a) Top view; (b) Front view; (c) Side view. 


\section{Computational Details}

\section{Methods}

Quantum mechanical calculations were performed with Gaussian 09. ${ }^{22}$ Ground state (GS) and transition state (TS) geometries were optimized in the gas phase using the B3LYP-D3 ${ }^{23-26}$ functional with the $6-31 \mathrm{G}(\mathrm{d})^{27,28}$ basis set. The D3 correction gave enantiomerization barriers that were in better agreement with those obtained experimentally, so these values were used throughout the discussion below and in the manuscript. Vibrational frequencies were computed to determine if the optimized structures are minima or saddle points on the potential energy surface corresponding to minima and TS geometries, respectively. The reported free energies include zero-point energies and thermal corrections calculated at $298.15 \mathrm{~K}$ and $1 \mathrm{~atm}$. Molecular structures were illustrated with CYLview. ${ }^{29}$

Due to the large number of side chains, the new compounds have many conformational degrees of freedom. Thus, we studied a model system in which the methoxymethyl groups were truncated to methyl groups, as these substituents are not expected to play a notable role in the enantiomerization processes. This truncation was made for each system. Figure S32 shows a representative comparison of the figure-eight dimer (2) with and without truncation.
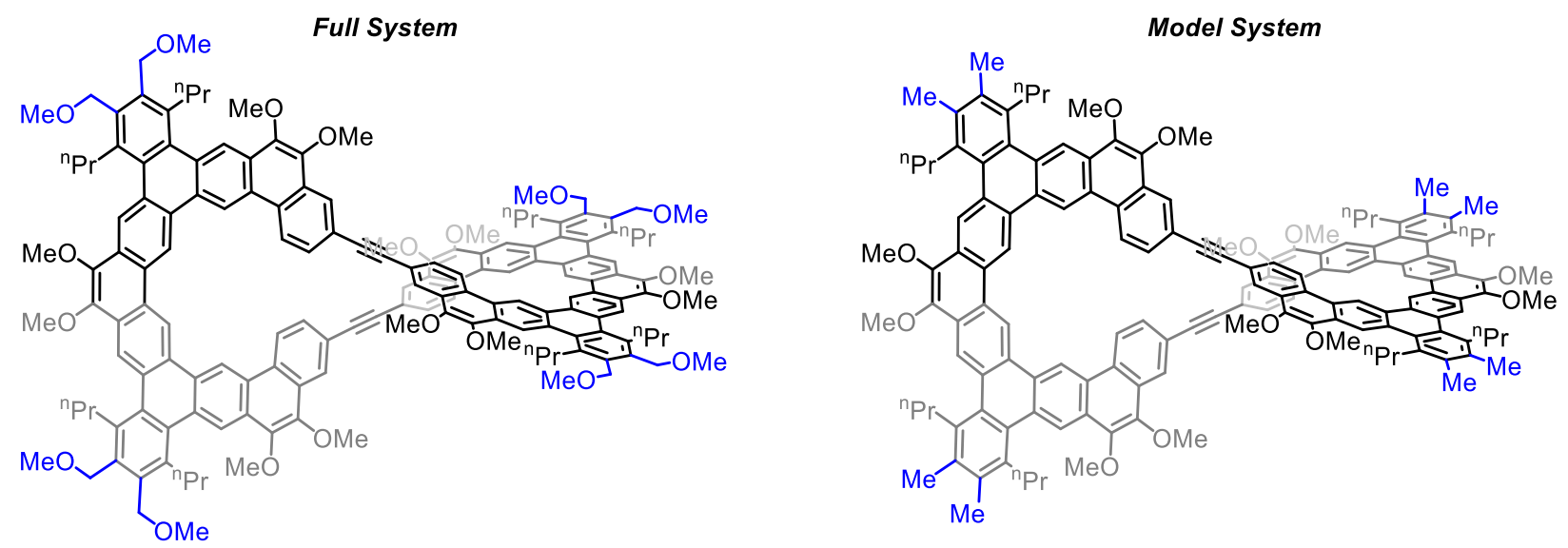

Figure S32. Model system for the figure-eight dimer 2, where the modified substituents are labeled in blue. 


\section{Phenylene-bridged expanded helicene (1-Benz)}

The geometries of the GS and enantiomerization TS for 1-Benz are shown in Figures S33 and S34, respectively. The predominant structural change during the enantiomerization process is the $\mathrm{C}_{1}-\mathrm{C}_{2}-\mathrm{C}_{3}-\mathrm{C}_{4}$ dihedral angle (shown in blue), which contracts from $-57^{\circ}$ in the $\mathrm{GS}$ to $14^{\circ}$ in the TS. This contraction, and the resulting coplanarity between the phenylene bridge and the neighboring helicene core, enforces an unfavorable steric interaction between the propyl group and the neighboring ring (similar to allylic $\mathrm{A}^{1,3}$ strain). The calculations also suggest that the enantiomerization of 1-Benz proceeds through a two-step mechanism (Figure S35) via a $C_{S^{-}}$ symmetric intermediate (1-Benz GS conf 2), which is $4.4 \mathrm{kcal} \mathrm{mol}^{-1}$ higher in energy than the lowest energy $C_{2}$ conformer (1-Benz GS). The overall calculated enantiomerization barrier for 1Benz was $\Delta \mathrm{G}^{\ddagger}=22.1 \mathrm{kcal} / \mathrm{mol}$.
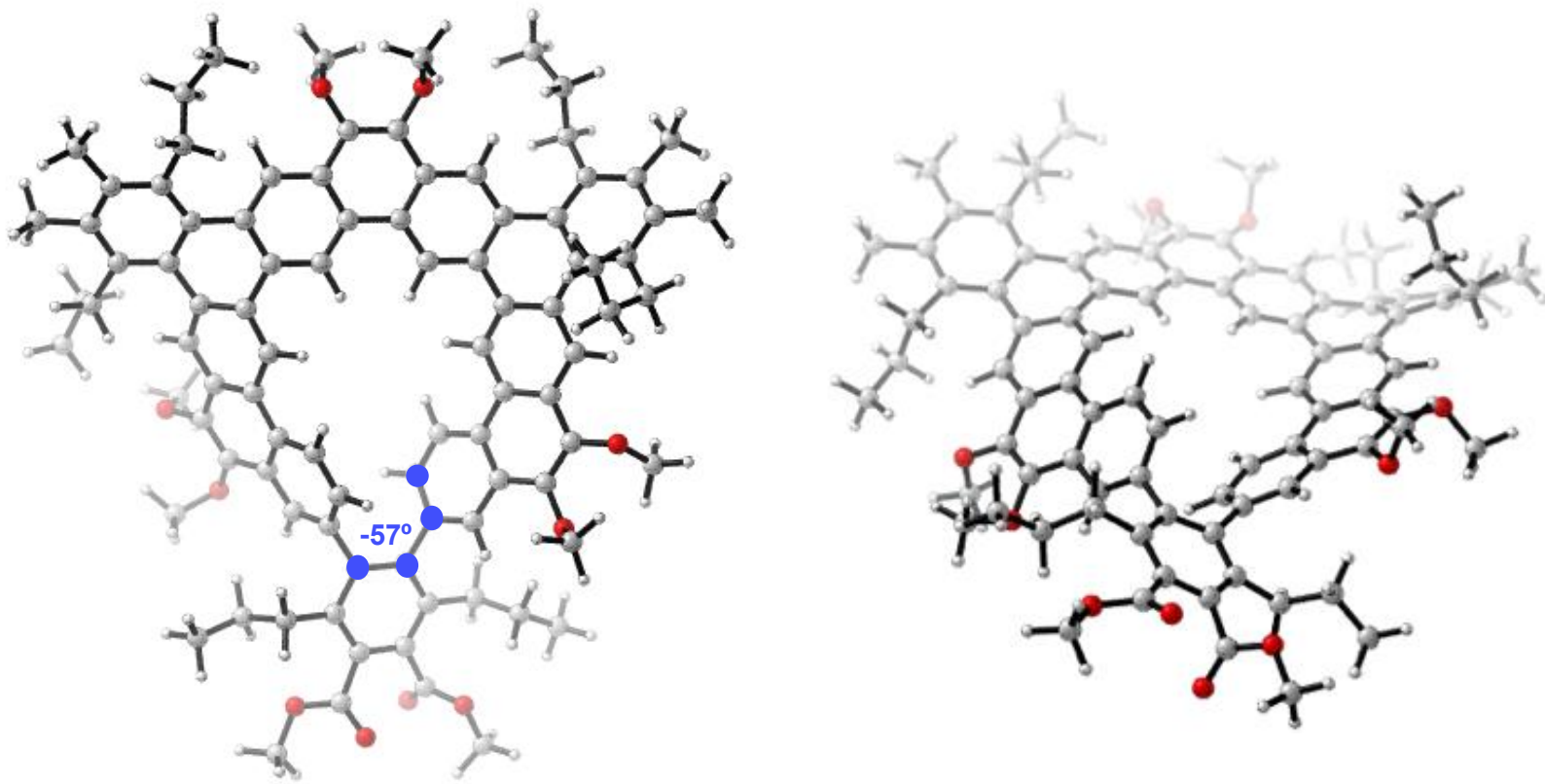

Figure S33. GS structures of 1-Benz from both the side and front views.
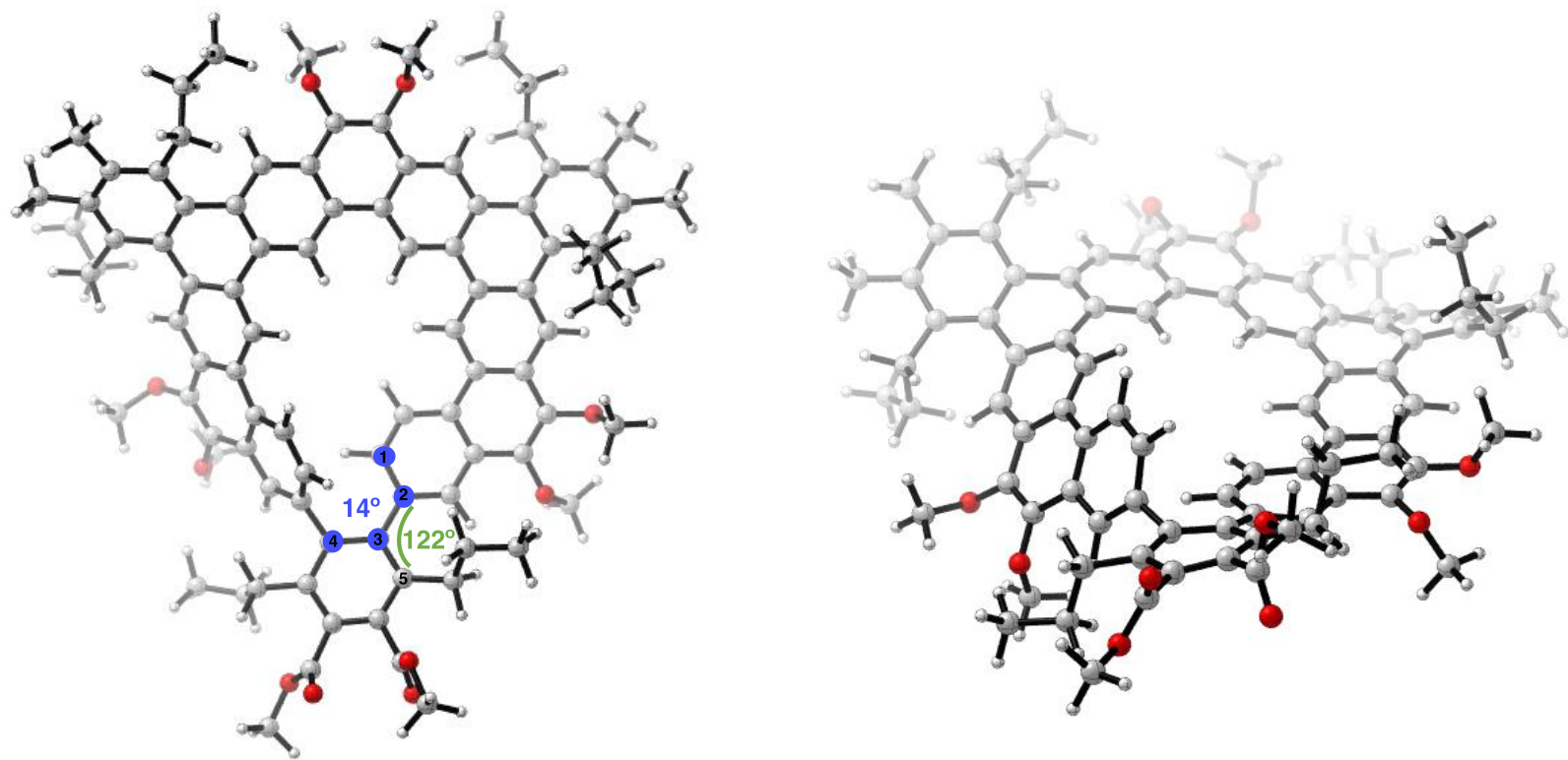

Figure S34. TS structures of 1-Benz from both the side and front views. 


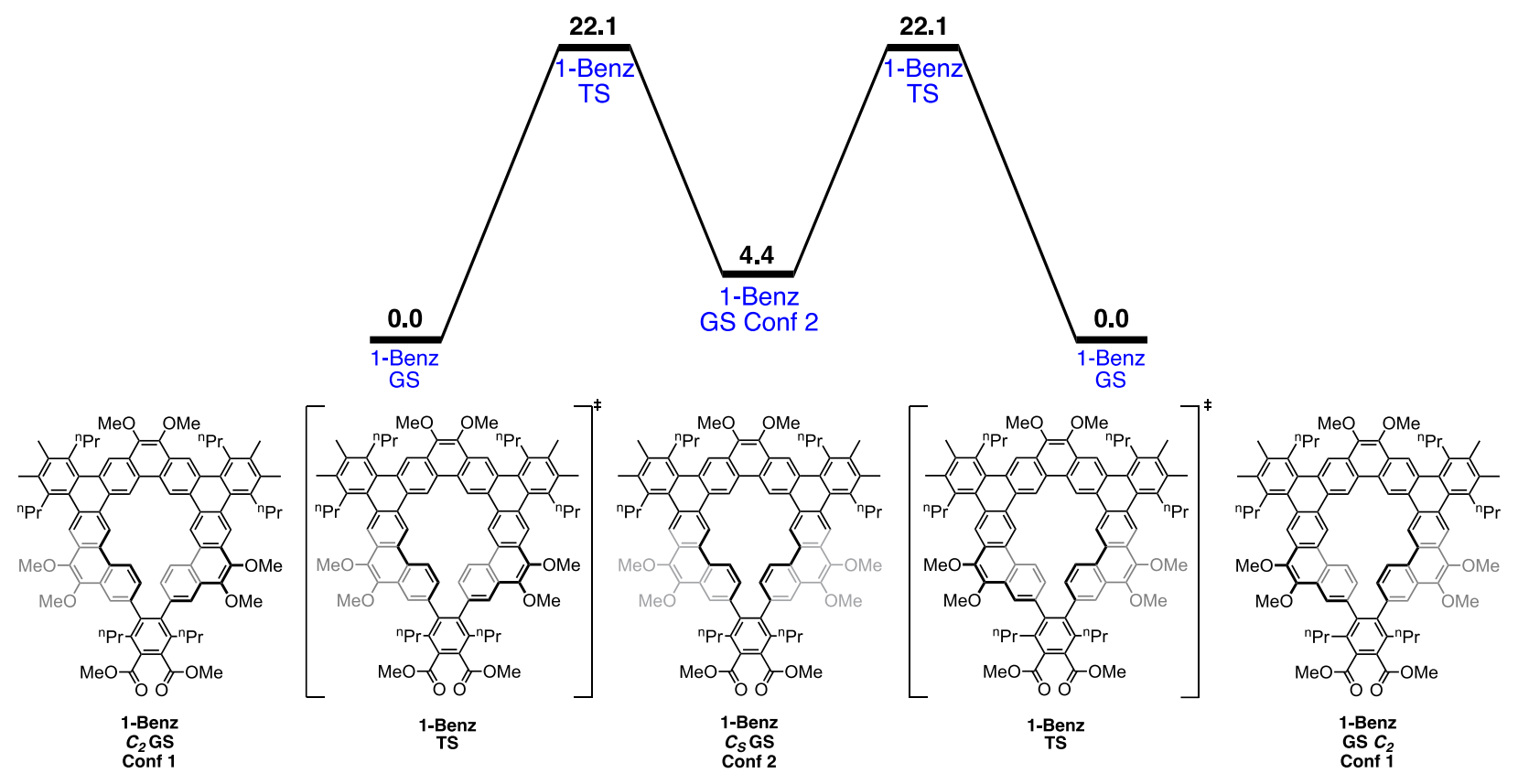

Figure S35. Two-step enantiomerization mechanism for 1-Benz. 


\section{Selenophene-bridged expanded helicene (1-Se)}

The GS geometry of $1-\mathrm{Se}$ is shown in Figure $\mathrm{S} 36$. The $\mathrm{C}_{1}-\mathrm{C}_{2}-\mathrm{C}_{3}-\mathrm{C}_{4}$ dihedral angle shown in blue is $-47^{\circ}$. Similar to 1-Benz, the enantiomerization TS structure of 1-Se in Figure S37 shows distortion of the noted dihedral angle to 15o, resulting in analogous unfavorable interactions (see discussion on 1-Benz above). These interactions are attenuated in 1-Se compared to 1-Benz, which appears to contribute to the large difference in their enantiomerization barriers (both computationally and experimentally). These calculations suggest that the enantiomerization of 1Se also proceeds through a two-step mechanism via a $C_{S}$-symmetric intermediate (1-Se GS conf 2), which is $9.9 \mathrm{kcal} \mathrm{mol}^{-1}$ higher in energy than the lowest energy $C_{2}$ conformer (1-Se GS). The full mechanism is shown in Figure S38 and the overall calculated enantiomerization barrier for 1Se was $\Delta \mathrm{G}^{\ddagger}=18.7 \mathrm{kcal} / \mathrm{mol}$.

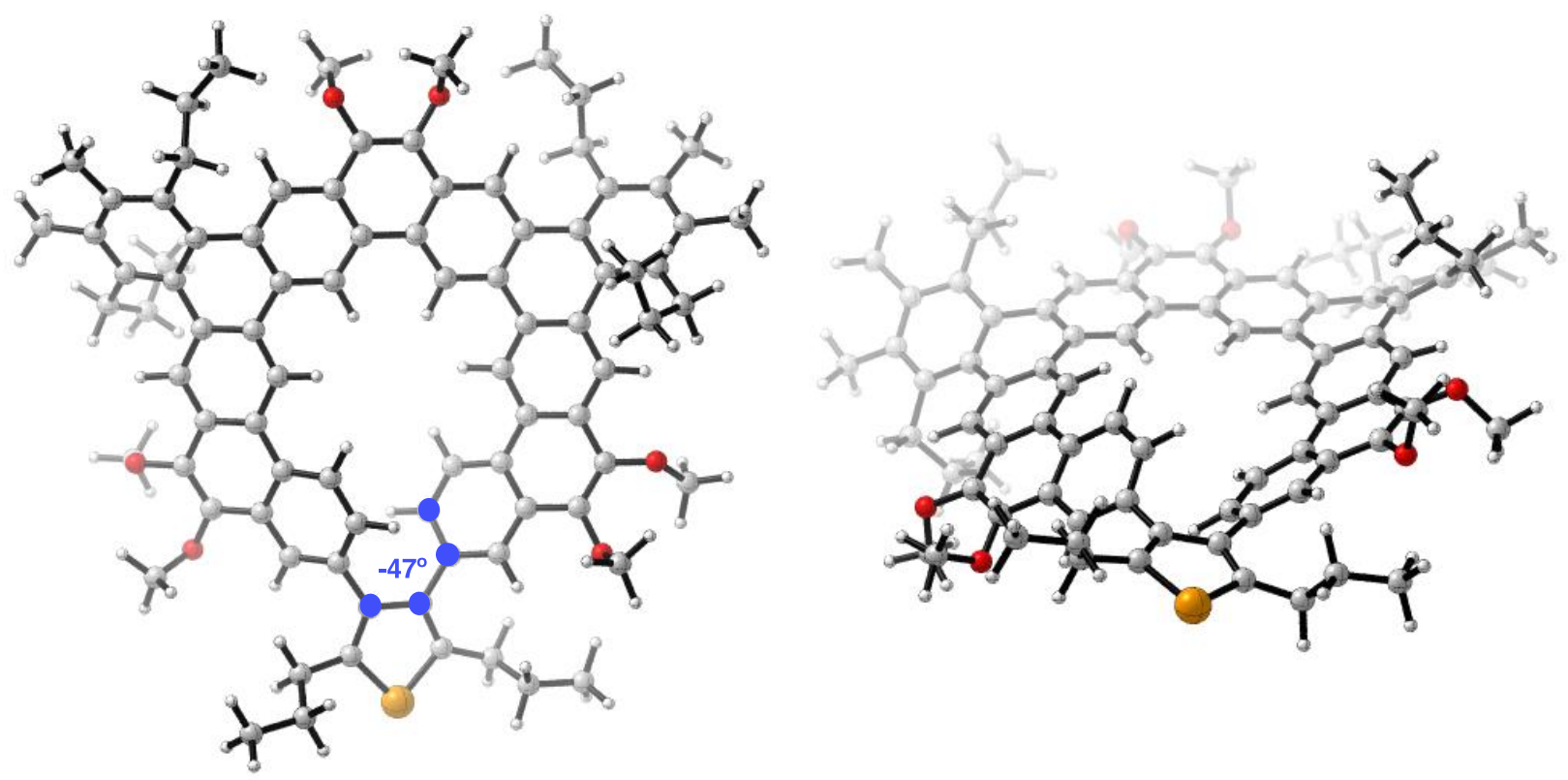

Figure S36. GS structures of 1-Se from both the side and front views.
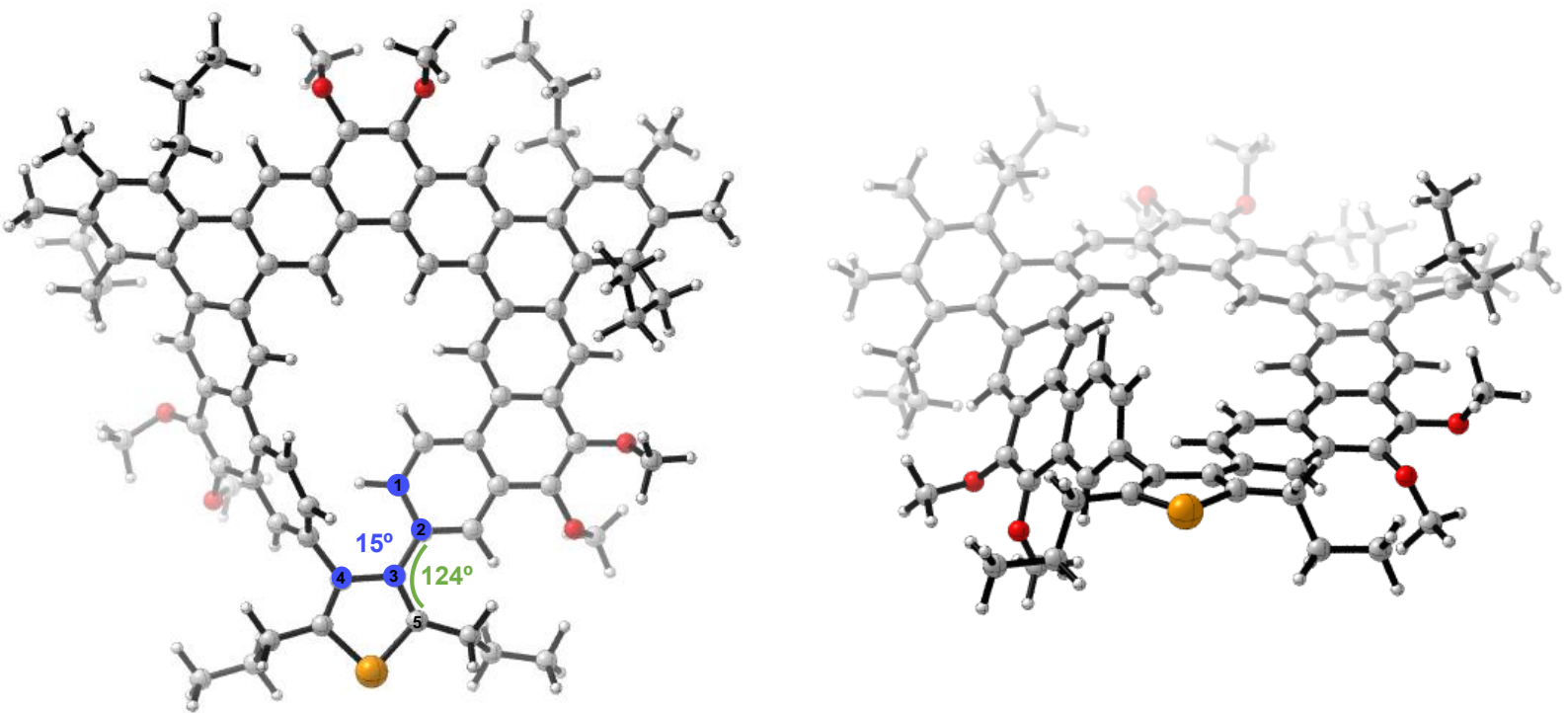

Figure S37. TS structures of 1-Se from both the side and front views. 


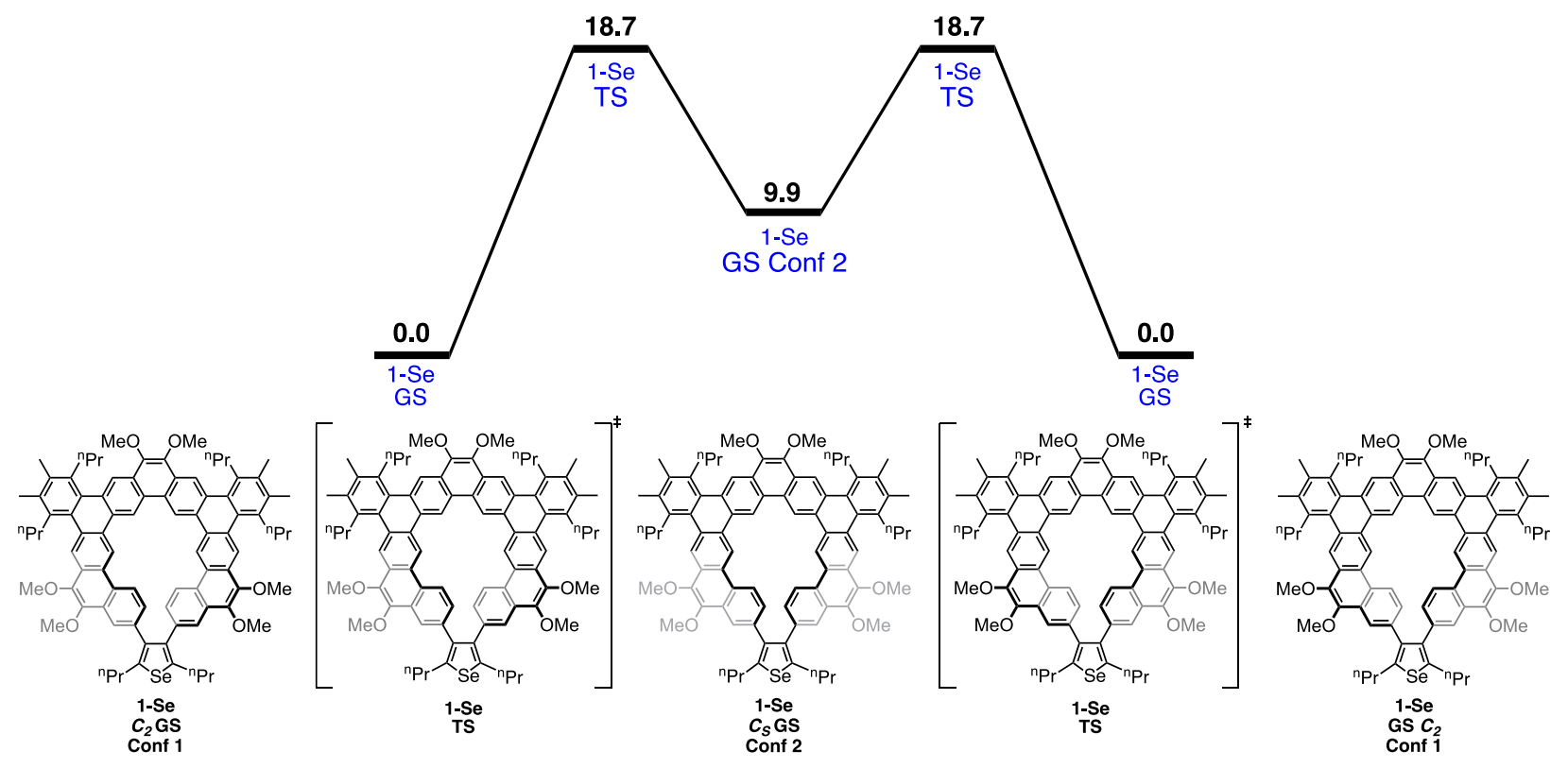

Figure S38. Two-step enantiomerization mechanism for 1-Se. 
Figure-eight dimer (2)

The GS geometry of the figure-eight dimer 2 is shown in Figure S39. The distance between the centroids of the alkynes is $3.60 \AA$ and the angle of the alkyne is $178^{\circ}$, which is close to the ideal angle of $180^{\circ}$. More comparative details of this particular structure to the experimentally determined x-ray crystal structure can be found in Figure S31 and the associated discussion.
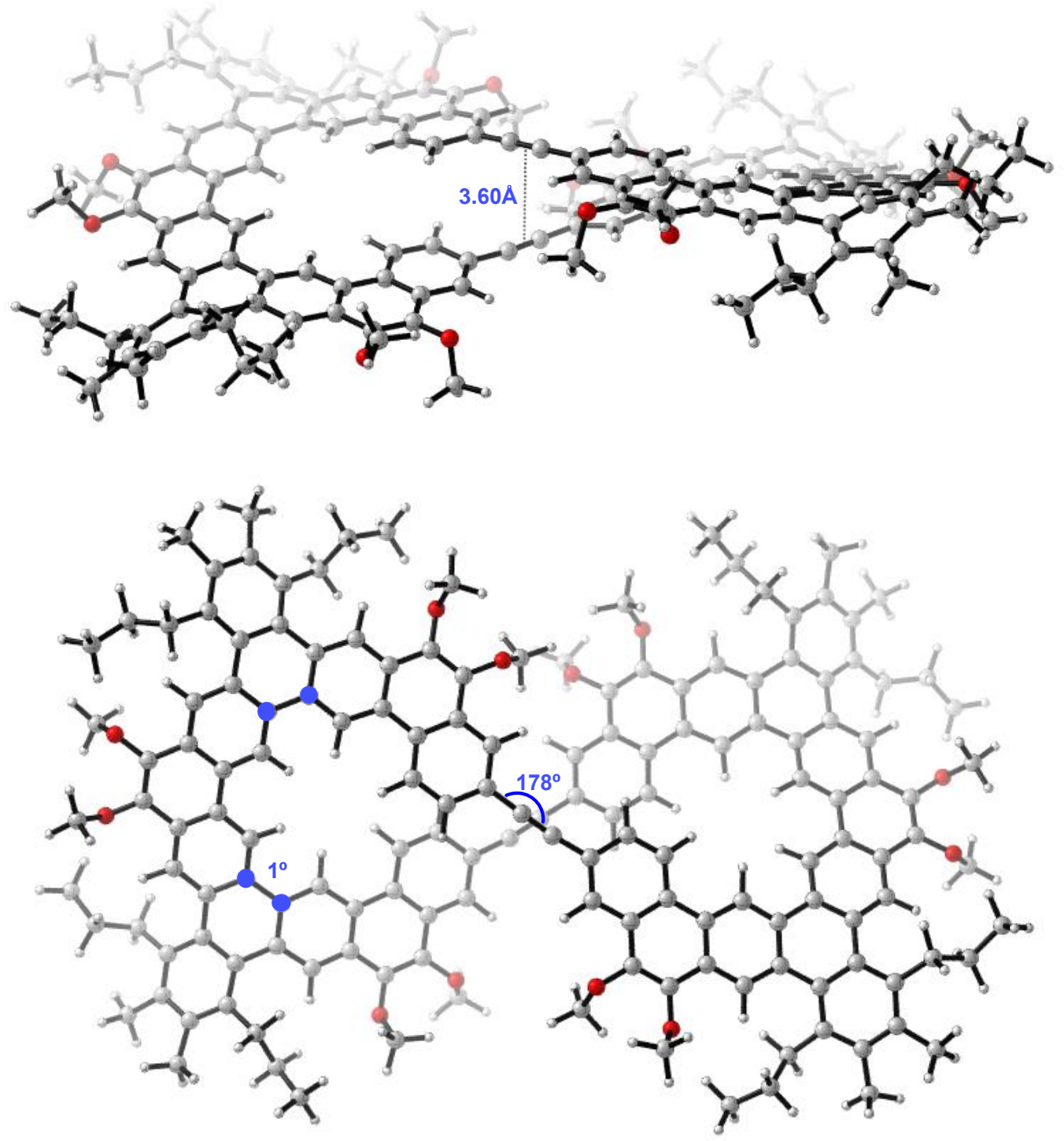

Figure S39. GS structures of the figure-eight dimer 2 from both the side and front views. 
The enantiomerization TS structure of figure-eight dimer 2 (Figure S40) shows distortion of the linear alkyne to $165^{\circ}$. The calculated enantiomerization barrier for 2 was $\Delta \mathrm{G}^{\ddagger}=15.5 \mathrm{kcal} / \mathrm{mol}$. The calculated IRC (irc=calcfc using the method B3LYP-D3/6-31(d)) starting from the TS of dimer 2 can be found in Figure S41.
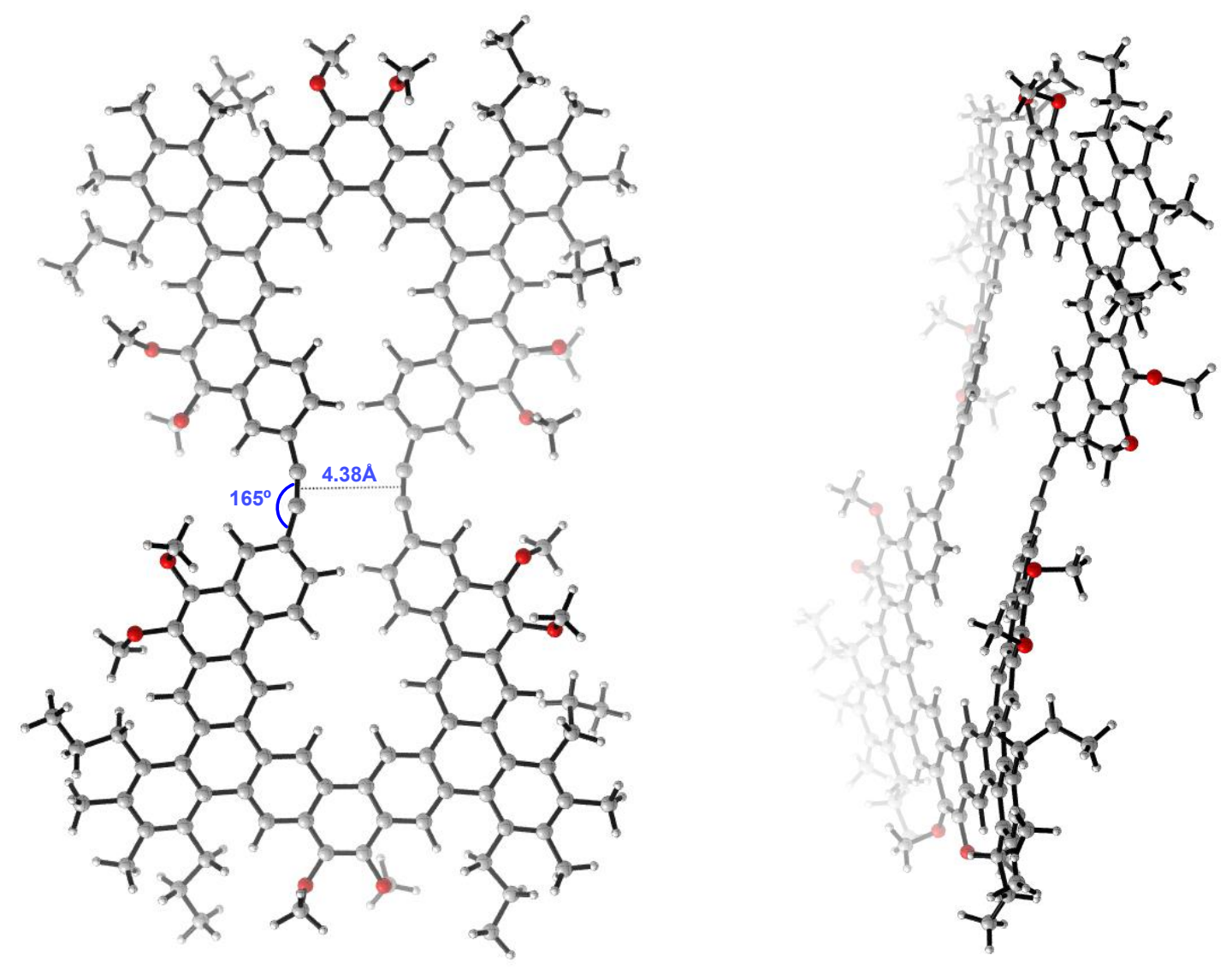

Figure S40. TS structures of the figure-eight dimer 2 from both the side and front views.

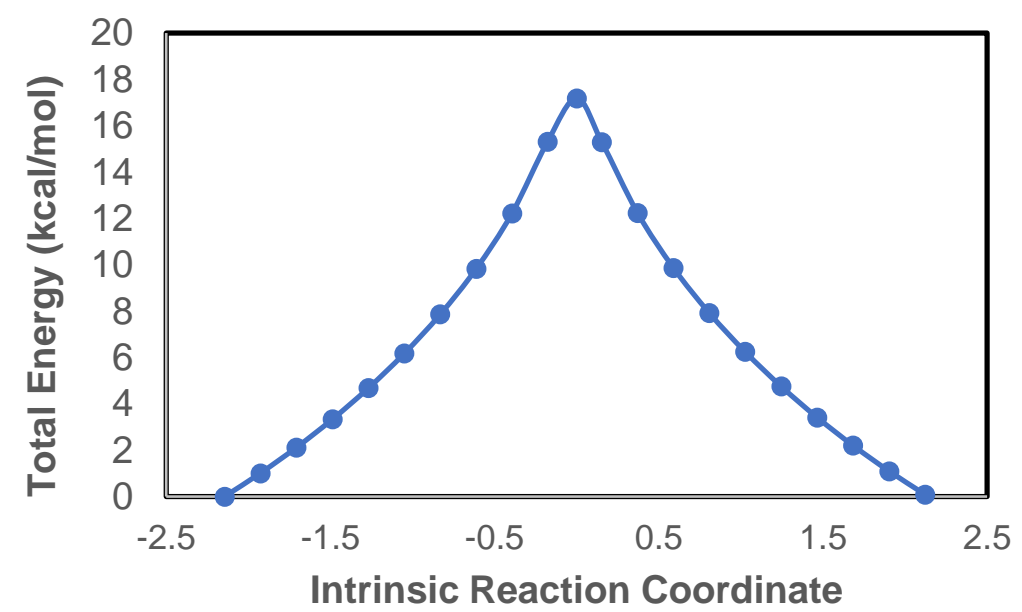

Figure S41. Intrinsic Reaction Coordinate (IRC) analysis for figure-eight dimer 2. 


\section{Alkynylated expanded helicene (3)}

The GS optimized geometry of helicene 3 is shown in Figure S42. The distance between the centroids of the alkynyl groups is 3.75 Angstroms. From the side view, the helicene has very little twist or distortion in the aromatic hydrocarbon core and remains relatively planar other than a small bend shown by the dihedral angle of $-4^{\circ}$ highlighted in blue. The bending occurs to relieve any steric interactions between the alkynyl arms of the helicene. The TS for helicene 3 (Figure S43) exhibits pseudo $C_{s}$ symmetry with the two alkynyl arms pointing above the plane of the helicene core. Compared to the GS, the distance between the centroids of the alkynyl groups contracts significantly (to 3.15 Angstroms). The calculated enantiomerization barrier for helicene 3 was $\Delta \mathrm{G}^{\ddagger}=14.1 \mathrm{kcal} / \mathrm{mol}$. The calculated IRC (irc=calcfc using the method B3LYP-D3/6-31(d)) starting from the TS of $\mathbf{3}$ can be found in Figure S44.
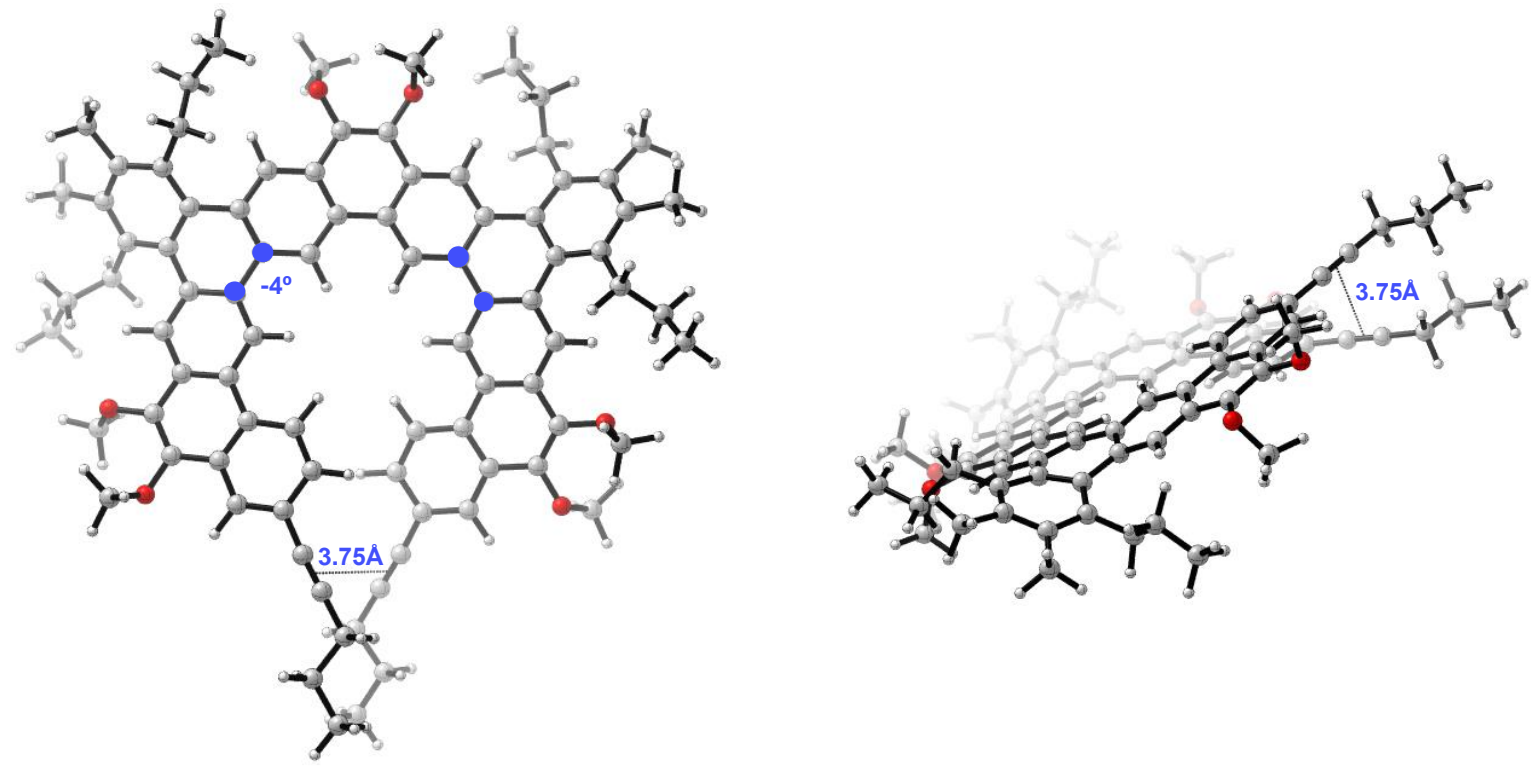

Figure S42. GS structures of helicene $\mathbf{3}$ from both the side and front views.
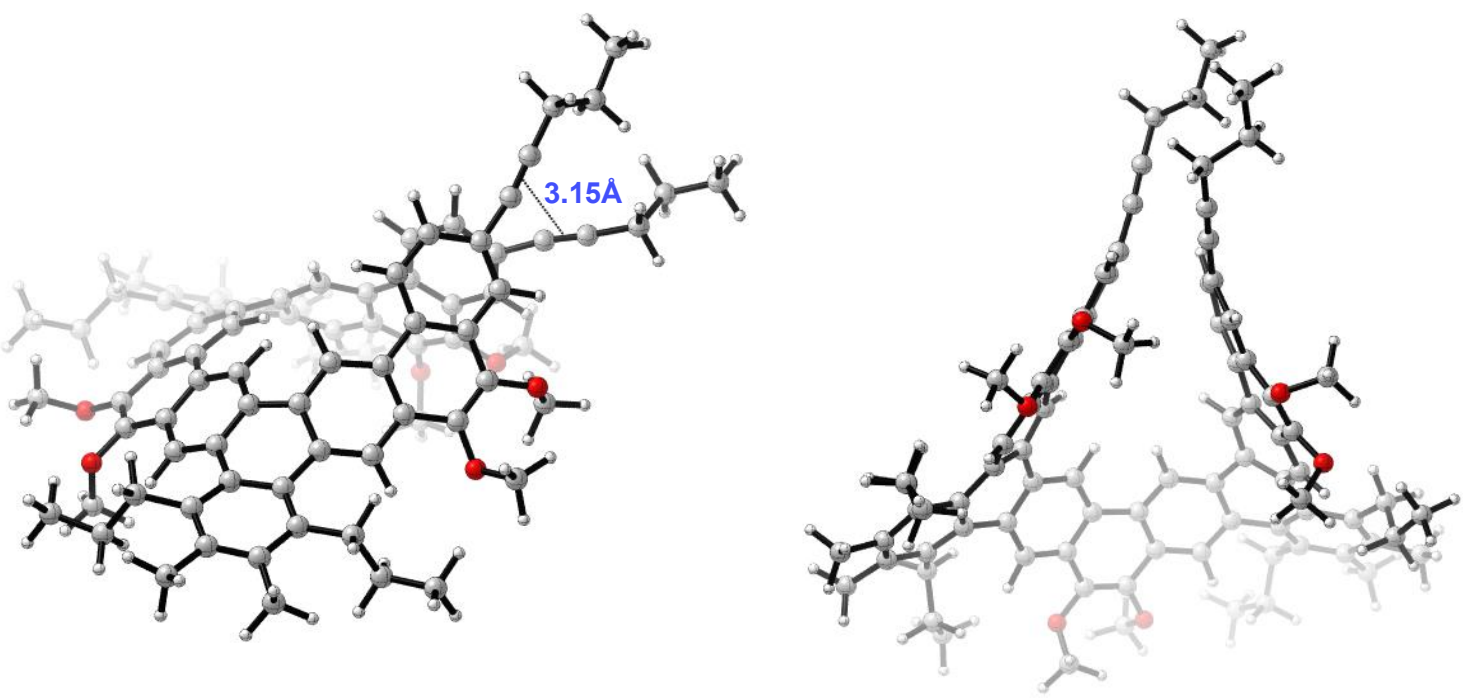

Figure S43. TS structures of helicene $\mathbf{3}$ from both the side and front views. 


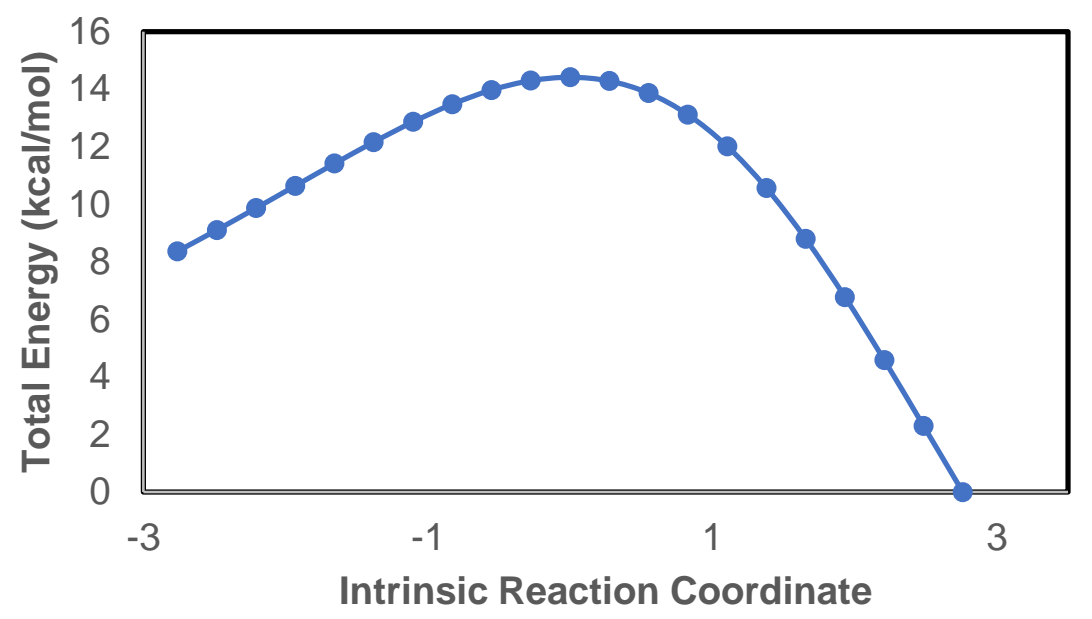

Figure S44. Intrinsic Reaction Coordinate (IRC) analysis for helicene 3. 


\section{Summary of energies and vibrational frequencies}

\begin{tabular}{|c|c|c|}
\hline Structure & B3LYP-D3/6-31 g(d) $\mathbf{( k c a l} / \mathbf{m o l})$ & B3LYP/6-31g(d) $(\mathbf{k c a l} / \mathbf{m o l})$ \\
\hline 1-Benz & 22.1 & 21.5 \\
\hline $1-S e$ & 18.7 & 17.4 \\
\hline 2 & 15.5 & 17.2 \\
\hline 3 & 14.1 & 15.3 \\
\hline
\end{tabular}

Table S4. Comparison of calculated enantiomerization free energies with and without dispersion correction (D3).

\begin{tabular}{|c|c|c|c|c|c|c|}
\hline Structure & E(gas) & ZPVE & TCE & TCH & TCG & $\begin{array}{c}\text { Neg } \\
\text { Freq } \\
\text { (cm-1) }\end{array}$ \\
\hline Helicene 3 GS & -3859.1217 & 1.566273 & 1.655628 & 1.656572 & 1.43808 & - \\
\hline Helicene 3 TS & -3859.1073 & 1.554867 & 1.592998 & 1.593942 & 1.448362 & -11.713 \\
\hline 1-Benz GS & -4313.7892 & 1.634986 & 1.730362 & 1.731306 & 1.500106 & - \\
\hline 1-Benz TS & -4313.7486 & 1.630805 & 1.727377 & 1.728321 & 1.493759 & -16.971 \\
\hline 1-Se GS & -6179.984 & 1.514361 & 1.600935 & 1.601879 & 1.390157 & - \\
\hline 1-Se TS & -6179.9502 & 1.510592 & 1.598222 & 1.599167 & 1.384034 & -20.672 \\
\hline Figure-8 Dimer 2 GS & -6934.499 & 2.694616 & 2.377718 & 2.378662 & 2.468869 & - \\
\hline Figure-8 Dimer 2 TS & -6934.5726 & 2.699459 & 2.377744 & 2.378688 & 2.569854 & -14.146 \\
\hline Helicene 3 GS & -3859.1217 & 1.566273 & 1.655628 & 1.656572 & 1.43808 & - \\
\hline Helicene 3 TS & -3859.1073 & 1.554867 & 1.592998 & 1.593942 & 1.448362 & -11.713 \\
\hline
\end{tabular}

Table S5. Energies and vibrational frequencies without D3 (B3LYP(gas) $/ 6-31 \mathrm{~g}(\mathrm{~d})$ ). E(gas) = gas phase energies; ZPVE = zero-point vibrational energy; TCE = thermal correction to energy; TCH $=$ thermal correction to enthalpy; TCG = thermal correction to Gibbs free energy.

\begin{tabular}{|l|c|c|c|c|c|c|}
\hline \multicolumn{1}{|c|}{ Structure } & E(gas) & ZPVE & TCE & TCH & TCG & $\begin{array}{c}\text { Neg } \\
\text { Freq } \\
\text { (cm-1) }\end{array}$ \\
\hline Helicene 3 GS & -3859.1217 & 1.566042 & 1.656591 & 1.657535 & 1.464366 & - \\
\hline Helicene 3 TS & -3859.073 & 1.566273 & 1.655628 & 1.656572 & 1.43808 & -8.148 \\
\hline 1-Benz GS & -4313.7797 & 1.635004 & 1.731232 & 1.732176 & 1.500882 & - \\
\hline 1-Benz GS conf 2 & -4313.7790 & 1.731333 & 1.732278 & 1.712371 & 1.493102 & - \\
\hline 1-Benz TS & -4313.7437 & 1.634986 & 1.730362 & 1.731306 & 1.500106 & -15.518 \\
\hline 1-Se GS & -6179.9756 & 1.513777 & 1.601372 & 1.602316 & 1.385595 & - \\
\hline 1-Se GS conf 2 & -6179.959 & 1.511936 & 1.599934 & 1.600878 & 1.384694 & - \\
\hline 1-Se TS & -6179.9504 & 1.514361 & 1.600935 & 1.601879 & 1.390157 & -19.589 \\
\hline Figure-8 Dimer 2 GS & -6934.4751 & 2.614351 & 2.768271 & 2.769215 & 2.420382 & - \\
\hline Figure-8 Dimer 2 TS & -6934.5706 & 2.663877 & 2.765097 & 2.766041 & 2.540568 & -9.381 \\
\hline
\end{tabular}

Table S6. Energies and vibrational frequencies with D3 (B3LYP-D3(gas)/6-31g(d)). E(gas) = Gas phase energies; ZPVE = zero-point vibrational energy; TCE = thermal correction to energy; TCH $=$ thermal correction to enthalpy; TCG $=$ thermal correction to Gibbs free energy. 


\begin{tabular}{|c|c|c|}
\hline Structure & Gas phase (kcal/mol) & In toluene (kcal/mol) \\
\hline 1-Benz & 22.1 & 23.9 \\
\hline 1-Se & 18.7 & 19.5 \\
\hline Figure-8 dimer 2 & 15.5 & 16.6 \\
\hline Helicene 3 & 14.1 & 16.1 \\
\hline
\end{tabular}

Table S7. Comparison of calculated gas phase and solution enantiomerization free energies. The latter were obtained from the single-point solvation energies shown in Table S8.

\begin{tabular}{|c|c|}
\hline Structure & E(toluene) \\
\hline Helicene 3 GS & -3859.1227 \\
\hline Helicene 3 TS & -3859.0707 \\
\hline 1-Benz GS & -4313.7755 \\
\hline 1-Benz TS & -4313.7367 \\
\hline 1-Se GS & -6179.9771 \\
\hline 1-Se TS & -6179.9505 \\
\hline Figure-8 Dimer GS & -6934.4775 \\
\hline Figure-8 Dimer TS & -6934.5713 \\
\hline
\end{tabular}

Table S8. Single-point solvation energies (B3LYP-D3(toluene)/6-31g(d)). E(toluene) = Single-point solvation energy. 


\section{Cartesian Coordinates}

\begin{tabular}{|c|c|c|c|}
\hline C & 4.27048000 & -0.72196100 & -0.16000900 \\
\hline C & 5.49163700 & -1.41110100 & -0.39814500 \\
\hline C & 4.28376700 & 0.70715000 & 0.09650500 \\
\hline $\mathrm{C}$ & 3.12191500 & 1.43849100 & 0.35811600 \\
\hline $\mathrm{C}$ & 3.12003100 & 2.81813800 & 0.55168600 \\
\hline C & 4.34477900 & 3.54148900 & 0.47967800 \\
\hline $\mathrm{C}$ & 5.50014500 & 2.82109700 & 0.16485300 \\
\hline $\mathrm{C}$ & 1.85468900 & 3.54862000 & 0.55586200 \\
\hline $\mathrm{C}$ & 1.88231700 & 4.91012600 & 0.10925500 \\
\hline $\mathrm{C}$ & 3.11513500 & 5.71146400 & 0.34269400 \\
\hline $\mathrm{C}$ & 4.32107800 & 5.00790200 & 0.67717600 \\
\hline $\mathrm{C}$ & 0.63659600 & 2.89531100 & 0.74145600 \\
\hline C & -0.57946500 & 3.42454800 & 0.28744100 \\
\hline $\mathrm{C}$ & 0.70403700 & 5.36304600 & -0.49037700 \\
\hline $\mathrm{C}$ & -1.85233500 & 2.72945600 & 0.39881800 \\
\hline $\mathrm{C}$ & 5.50775200 & 1.42449300 & 0.00758300 \\
\hline $\mathrm{C}$ & -0.50349600 & 4.64177900 & -0.44769100 \\
\hline $\mathrm{C}$ & -2.95206800 & 3.18833200 & -0.37970700 \\
\hline $\mathrm{C}$ & -4.15312000 & 2.45043200 & -0.39098100 \\
\hline $\mathrm{C}$ & -2.06315900 & 1.60594200 & 1.23087600 \\
\hline $\mathrm{C}$ & 6.72193700 & -0.65867400 & -0.43506500 \\
\hline C & -2.83431700 & 4.40701200 & -1.14345400 \\
\hline $\mathrm{C}$ & -3.27383600 & 0.93517100 & 1.25598000 \\
\hline C & 6.72599500 & 0.70224500 & -0.26853200 \\
\hline $\mathrm{C}$ & -1.66386500 & 5.11720100 & -1.15350000 \\
\hline $\mathrm{C}$ & -4.33575800 & 1.33266800 & 0.41336200 \\
\hline $\mathrm{H}$ & 2.17040800 & 0.92669600 & 0.32615400 \\
\hline $\mathrm{H}$ & 6.43380400 & 3.33236800 & -0.00046800 \\
\hline $\mathrm{H}$ & 0.64344800 & 1.90155000 & 1.17380900 \\
\hline $\mathrm{H}$ & 0.68182500 & 6.27873100 & -1.05178800 \\
\hline $\mathrm{H}$ & -4.94063900 & 2.76406400 & -1.06456900 \\
\hline $\mathrm{H}$ & -1.26196300 & 1.26305500 & 1.87820900 \\
\hline $\mathrm{H}$ & -3.39892900 & 0.06871800 & 1.89801000 \\
\hline C & 5.43031200 & 5.70527500 & 1.23065500 \\
\hline C & 5.39927200 & 7.10992600 & 1.28434200 \\
\hline C & 4.27991900 & 7.81559800 & 0.80270000 \\
\hline C & 3.10980300 & 7.13608300 & 0.41416300 \\
\hline C & -5.62502500 & 0.57889600 & 0.28385100 \\
\hline C & -6.82882000 & 1.19482900 & 0.69757000 \\
\hline C & -8.04242900 & 0.54620900 & 0.39805400 \\
\hline C & -8.04825500 & -0.66501600 & -0.31544200 \\
\hline C & -6.85970400 & -1.31297600 & -0.67706000 \\
\hline C & -5.63408100 & -0.70349800 & -0.31669800 \\
\hline C & -9.37695900 & -1.17968700 & -0.80520400 \\
\hline C & -9.38352800 & 1.03027900 & 0.86707900 \\
\hline $\mathrm{O}$ & -10.04924200 & -0.60399000 & -1.63463800 \\
\hline $\mathrm{O}$ & -10.24942800 & 0.29418100 & 1.29929100 \\
\hline C & -6.92330300 & -2.56021700 & -1.55094400 \\
\hline $\mathrm{H}$ & -7.73241000 & -2.40342400 & -2.27669700 \\
\hline $\mathrm{H}$ & -6.00421500 & -2.63332400 & -2.14149500 \\
\hline C & -6.79759500 & 2.45753600 & 1.54853700 \\
\hline $\mathrm{H}$ & -5.86115500 & 2.47788500 & 2.11703200 \\
\hline $\mathrm{H}$ & -7.60294700 & 2.39365300 & 2.28808700 \\
\hline C & 3.09688000 & -1.47848300 & -0.15939200 \\
\hline C & 3.08397200 & -2.84921100 & -0.39297500 \\
\hline C & 4.30293300 & -3.55906700 & -0.58282600 \\
\hline C & 5.48344900 & -2.80809100 & -0.56048200 \\
\hline C & 1.83194800 & -3.58704900 & -0.33955500 \\
\hline C & 1.86831100 & -4.96391300 & 0.03614700 \\
\hline C & 3.16663900 & -5.68144100 & 0.02370600 \\
\hline C & 4.27050900 & -5.03888200 & -0.62277300 \\
\hline C & 0.61970500 & -2.94857500 & -0.58777800 \\
\hline C & -0.61501700 & -3.55583100 & -0.33908800 \\
\hline
\end{tabular}

$\begin{array}{lll}0.63243700 & -5.57601600 & 0.26692000\end{array}$ $\begin{array}{lll}-1.88277500 & -2.86633000 & -0.49971800\end{array}$ $\begin{array}{lll}-0.58886900 & -4.88670300 & 0.16881200\end{array}$ $\begin{array}{lll}-3.04108000 & -3.43681100 & 0.09378100\end{array}$ $-2.03145800-1.63656400-1.17980200$ $\begin{array}{lll}-4.35535100 & -1.45759500 & -0.49268800\end{array}$ $\begin{array}{lll}-4.23326100 & -2.68844900 & 0.14155300\end{array}$ $\begin{array}{llll}-3.23922500 & -0.96344900 & -1.20668900\end{array}$ $\begin{array}{lll}-2.98232100 & -4.77093300 & 0.63911200\end{array}$

$\begin{array}{lll}-1.81992200 & -5.49670800 & 0.60558000\end{array}$ $\begin{array}{llll}2.15833800 & -0.99685300 & 0.07971500\end{array}$ $6.44465300-3.28872500-0.62628700$ $\begin{array}{llll}0.63947000 & -1.92650100 & -0.94653500\end{array}$ $\begin{array}{lll}0.57059000 & -6.60999000 & 0.55765400\end{array}$ $\begin{array}{lll}-5.06594600 & -3.08782300 & 0.70443200\end{array}$ $\begin{array}{llll}5.29979000 & -5.83384400 & -1.19728600\end{array}$ $\begin{array}{lll}5.32134500 & -7.21501500 & -0.93797500\end{array}$ $\begin{array}{llll}3.35636300 & -7.00695100 & 0.51217700\end{array}$ $\begin{array}{lll}4.43488600 & -7.76327200 & 0.02023900\end{array}$ $\begin{array}{lll}-3.31868400 & -0.02429600 & -1.74327900\end{array}$ $\begin{array}{lll}-1.18500200 & -1.20931100 & -1.70795800\end{array}$ $\begin{array}{lll}-9.72485800 & -2.34914000 & -0.24008900\end{array}$ $\begin{array}{lll}-9.55719800 & 2.36163900 & 0.74478300\end{array}$ $\begin{array}{lll}-11.01226100 & -2.85384200 & -0.63366700\end{array}$ $\begin{array}{llll}-11.12995100 & -3.79711600 & -0.09976100\end{array}$ $\begin{array}{lll}-11.79313400 & -2.14467300 & -0.34650400\end{array}$ $\begin{array}{lll}-11.05015500 & -3.01408600 & -1.71488200\end{array}$ $\begin{array}{lll}-10.84086200 & 2.85223700 & 1.16791000\end{array}$ $\begin{array}{lll}-10.80694800 & 3.92949800 & 1.00289200\end{array}$ $\begin{array}{lll}-11.63606900 & 2.39247800 & 0.57521200\end{array}$ $\begin{array}{lll}-11.00910200 & 2.62719600 & 2.22482800\end{array}$ $\begin{array}{lll}-4.16112600 & -5.30215000 & 1.11759900\end{array}$ $\begin{array}{lll}-1.75710900 & -6.79253000 & 1.07257600\end{array}$ $\begin{array}{llll}-3.93717300 & 4.81517700 & -1.86171300\end{array}$ $\begin{array}{llll}-1.55666500 & 6.31187800 & -1.83668200\end{array}$ $\begin{array}{lll}-2.50268800 & -7.74250800 & 0.29879800\end{array}$ $\begin{array}{llll}-2.15360800 & -7.75114200 & -0.74148200\end{array}$ $\begin{array}{lll}-2.31390200 & -8.71718200 & 0.75480800\end{array}$ $\begin{array}{lll}-3.57423700 & -7.51963800 & 0.32224700\end{array}$ $\begin{array}{lll}-4.16792400 & -5.59862600 & 2.52092200\end{array}$ $\begin{array}{lll}-3.95249600 & -4.69583800 & 3.10626700\end{array}$ $\begin{array}{lll}-5.17698500 & -5.94884800 & 2.74951500\end{array}$ $\begin{array}{lll}-3.43602500 & -6.37493000 & 2.76533800\end{array}$ $\begin{array}{llll}-4.54480800 & 6.03243600 & -1.40483900\end{array}$ $\begin{array}{lll}-5.44148900 & 6.16726600 & -2.01390900\end{array}$ $\begin{array}{llll}-4.83146200 & 5.94982700 & -0.34916200\end{array}$ $\begin{array}{lll}-3.86956700 & 6.88356700 & -1.53431400\end{array}$ $\begin{array}{lll}-1.51385400 & 6.17870100 & -3.26383700\end{array}$ $\begin{array}{llll}-2.44808500 & 5.75289900 & -3.64466200\end{array}$ $\begin{array}{lll}-1.37335900 & 7.18818500 & -3.65686500\end{array}$ $\begin{array}{llll}-0.66929100 & 5.54677700 & -3.56618700\end{array}$ $\begin{array}{lll}2.50611300 & -7.59597500 & 1.63893500\end{array}$ $\begin{array}{lll}1.53370000 & -7.95552300 & 1.28273300\end{array}$ $\begin{array}{llll}3.00326800 & -8.49210800 & 2.01311900\end{array}$ $\begin{array}{llll}6.32373200 & -5.23273700 & -2.15110700\end{array}$ $6.00129100-4.23787600-2.46454400$ $\begin{array}{lll}6.34198600 & -5.82373500 & -3.07609700\end{array}$ $\begin{array}{llll}6.62057100 & 4.98266000 & 1.85086800\end{array}$ $\begin{array}{lll}6.36728100 & 3.94024900 & 2.05032200\end{array}$ $\begin{array}{lll}6.82014300 & 5.41561600 & 2.83944300\end{array}$ $\begin{array}{lll}1.87339800 & 7.98784400 & 0.16890900\end{array}$ $\begin{array}{llll}0.97770800 & 7.45247900 & 0.49134500\end{array}$ $\begin{array}{llll}1.91143600 & 8.87369400 & 0.81139000\end{array}$ $\begin{array}{lll}7.88450700 & -1.35297300 & -0.70089000\end{array}$ $\begin{array}{llll}7.89964400 & 1.42643900 & -0.29843600\end{array}$ 


\begin{tabular}{|c|c|c|c|}
\hline 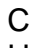 & 8.79782100 & -1.44010000 & 0.40055000 \\
\hline $\mathrm{H}$ & 9.62878900 & -2.05877500 & 0.05390200 \\
\hline $\mathrm{H}$ & 9.16160000 & -0.44846500 & 0.68899200 \\
\hline $\mathrm{H}$ & 8.31749700 & -1.92112600 & 1.26205300 \\
\hline C & 8.52707600 & 1.51478500 & -1.58433900 \\
\hline $\mathrm{H}$ & 9.39971800 & 2.15781600 & -1.44861300 \\
\hline $\mathrm{H}$ & 8.83901000 & 0.52732600 & -1.93922000 \\
\hline $\mathrm{H}$ & 7.84627200 & 1.97039100 & -2.31447700 \\
\hline C & -7.17348700 & -3.91459400 & -0.84194800 \\
\hline $\mathrm{H}$ & -6.23276700 & -4.31546300 & -0.45379900 \\
\hline $\mathrm{H}$ & -7.83543400 & -3.76241000 & 0.01452700 \\
\hline 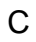 & -7.79644500 & -4.93756000 & -1.79662300 \\
\hline $\mathrm{H}$ & -8.78334100 & -4.60316500 & -2.14080100 \\
\hline $\mathrm{H}$ & -7.16860000 & -5.08672600 & -2.68419100 \\
\hline $\mathrm{H}$ & -7.92159600 & -5.91148600 & -1.30945700 \\
\hline & -6.94380400 & 3.79709000 & 0.79456500 \\
\hline & -6.00581100 & 4.02576700 & 0.28305600 \\
\hline & -7.71361500 & 3.70192400 & 0.02433700 \\
\hline & -7.29838300 & 4.94358700 & 1.74452500 \\
\hline & -8.26995400 & 4.76847000 & 2.22324700 \\
\hline & -6.55007000 & 5.04793600 & 2.54050200 \\
\hline & -7.35447700 & 5.90037100 & 1.21179400 \\
\hline C & 2.31422200 & -6.66919600 & 2.85874200 \\
\hline $\mathrm{H}$ & 1.75016200 & -5.77420900 & 2.58221100 \\
\hline $\mathrm{H}$ & 1.69906900 & -7.20653900 & 3.59291200 \\
\hline C & 3.64331000 & -6.25800500 & 3.50166700 \\
\hline $\mathrm{H}$ & 4.22237400 & -7.13692500 & 3.81357400 \\
\hline $\mathrm{H}$ & 4.25870200 & -5.68612300 & 2.79879500 \\
\hline & 3.47678700 & -5.63506000 & 4.38773100 \\
\hline & 7.76501200 & -5.15393900 & -1.59931600 \\
\hline & 7.74912200 & -4.76759800 & -0.57205300 \\
\hline & 8.18369900 & -6.16402900 & -1.52242100 \\
\hline & 8.67024200 & -4.27200700 & -2.46424900 \\
\hline $\mathrm{H}$ & 8.67019900 & -4.60973600 & -3.50831000 \\
\hline & 8.33172400 & -3.23012200 & -2.44051300 \\
\hline & 9.70678200 & -4.29549300 & -2.10698300 \\
\hline & 4.64115400 & -9.18942700 & 0.50484800 \\
\hline & 4.90837800 & -9.20940900 & 1.56900600 \\
\hline & 3.73783500 & -9.79958200 & 0.38939900 \\
\hline & 5.44352400 & -9.69967200 & -0.02599300 \\
\hline & 6.31160200 & -8.12923600 & -1.63746100 \\
\hline & 6.76257500 & -7.66378400 & -2.51365200 \\
\hline & 7.13398300 & -8.43093400 & -0.97557400 \\
\hline & 5.82341600 & -9.04626500 & -1.98344500 \\
\hline & 7.92857600 & 5.03930300 & 1.03060600 \\
\hline & 7.72461700 & 4.78348900 & -0.01695800 \\
\hline & 8.30496100 & 6.06855300 & 1.00797600 \\
\hline & 9.00111100 & 4.09965100 & 1.58876100 \\
\hline & 9.19916800 & 4.31183600 & 2.64709500 \\
\hline & 8.68284000 & 3.05535800 & 1.50038400 \\
\hline & 9.94700500 & 4.20860400 & 1.04476700 \\
\hline & 1.70030700 & 8.46187300 & -1.29086500 \\
\hline & 2.53865500 & 9.11706700 & -1.55913400 \\
\hline & 1.77821100 & 7.60594200 & -1.97320500 \\
\hline & 0.37045200 & 9.18817600 & -1.51400100 \\
\hline & -0.47508200 & 8.51490700 & -1.33383100 \\
\hline & 0.27285800 & 10.04593100 & -0.83661800 \\
\hline & 0.29065400 & 9.56461300 & -2.54085500 \\
\hline & 6.53828000 & 7.91235800 & 1.88765000 \\
\hline & 6.16114200 & 8.72597100 & 2.51533300 \\
\hline & 7.19852200 & 7.31090100 & 2.51091500 \\
\hline & 7.16253100 & 8.37349700 & 1.11028200 \\
\hline & 4.34153600 & 9.33403600 & 0.79755400 \\
\hline & 5.34118700 & 9.68583100 & 0.52785500 \\
\hline & 3.65107500 & 9.78398600 & 0.08530700 \\
\hline & 4.11149700 & 9.75636700 & 1.78637600 \\
\hline
\end{tabular}

1-Benz GS conf 2

C $\quad-4.34894900 \quad-0.58037100 \quad-0.79343800$

C $\quad-5.58145700 \quad-1.23285000-0.49257100$

C $\quad-4.30903600 \quad 0.87915800 \quad-0.82390500$

C $\quad-3.12452300 \quad 1.61609800 \quad-0.95213100$

C $\quad-3.05698500 \quad 2.99895600 \quad-0.76018100$

$\begin{array}{llll}\text { C } & -4.25151700 & 3.73719300 & -0.48724300\end{array}$

C $\quad \begin{array}{llll}\text { C } & -5.43226800 & 3.00452800 & -0.36887000\end{array}$

$\begin{array}{llll}\text { C } & -1.75843600 & 3.62447400 & -0.49540800\end{array}$

$\begin{array}{llll}\text { C } & -1.77343800 & 4.80888400 & 0.30293500\end{array}$

C $\quad-2.91944900 \quad 5.73268300 \quad 0.17118000$

$\begin{array}{llll}\text { C } & -4.17385500 & 5.20236200 & -0.26549300\end{array}$

C $\quad-0.54105500 \quad 2.99189300 \quad-0.73741400$

$\begin{array}{llll}\text { C } & 0.61865200 & 3.32070300 & -0.01952700\end{array}$

C $\quad \begin{array}{llll}\text { C } & -0.69684700 & 4.99602500 & 1.17125400\end{array}$

C $\quad 1.89570100 \quad 2.67458000 \quad-0.24881000$

C $\quad-5.49479400 \quad 1.61228700 \quad-0.53522800$

C $\quad 0.48148900 \quad 4.23322600 \quad 1.06628300$

$\begin{array}{llll}\text { C } & 2.89434500 & 2.73389600 & 0.76231100\end{array}$

$\begin{array}{llll}\text { C } & 4.08905800 & 1.99742900 & 0.60536300\end{array}$

C $\quad 2.21190900 \quad 2.01929800-1.45560800$

C $\quad-6.78053500 \quad-0.45248700-0.31792900$

C $\quad 2.68362700 \quad 3.58220200 \quad 1.90853400$

C $\quad 3.42861800 \quad 1.38902600 \quad-1.62704800$

$\begin{array}{lllll}\text { C } & -6.73969200 & 0.91347100 & -0.33813500\end{array}$

$\begin{array}{llll}\text { C } & 1.54888700 & 4.34347800 & 2.02598100\end{array}$

C $\quad 4.36272700 \quad 1.31162900 \quad-0.57047800$

$\begin{array}{llll}\mathrm{H} & -2.19537400 & 1.07687900 & -1.07985600\end{array}$

$\begin{array}{llll}\mathrm{H} & -6.34558300 & 3.48623100 & -0.06810800\end{array}$

H $\quad-0.50503400 \quad 2.15571800 \quad-1.42687800$

$\begin{array}{llll}\mathrm{H} & -0.75222200 & 5.73274100 & 1.96276200\end{array}$

$\begin{array}{llll}H & 4.80122200 & 1.98783100 & 1.42267000\end{array}$

$\mathrm{H} \quad \begin{array}{llll}\mathrm{H} & 1.50509100 & 2.04637200 & -2.27936800\end{array}$

$\begin{array}{llll}\mathrm{H} & 3.67306500 & 0.94477800 & -2.58531700\end{array}$

$\begin{array}{llll}\text { C } & -5.26152900 & 6.08573700 & -0.51790700\end{array}$

$\begin{array}{llll}\text { C } & -5.12722400 & 7.44689700 & -0.18213400\end{array}$

$\begin{array}{llll}\text { C } & -3.89698300 & 7.95884100 & 0.27788700\end{array}$

$\begin{array}{llll}\text { C } & -2.75887900 & 7.13436300 & 0.34427100\end{array}$

C $\quad 5.63290000 \quad 0.53163800-0.73181600$

C $\quad 6.84667000 \quad 1.21655900-0.95376400$

C $\quad 8.02784600 \quad 0.45832600 \quad-1.05214400$

$\begin{array}{llll}\text { C } & 7.99628200 & -0.93850900 & -0.92750900\end{array}$

C $\quad 6.79103800 \quad-1.62985600 \quad-0.74416200$

C $\quad 5.60005400-0.88052000-0.65136900$

C $\quad 9.28566600-1.69186900-1.11418500$

C $\quad 9.38525100 \quad 1.08153700-1.21111800$

O $\quad 9.87659900-1.76890400-2.17093500$

$\begin{array}{llll}O & 10.33535000 & 0.81671300 & -0.50147200\end{array}$

C $\quad 6.77361100-3.14563000 \quad-0.78448600$

$\mathrm{H} \quad 7.68690100 \quad-3.54193600 \quad-0.33053800$

$\mathrm{H} \quad 5.93948100-3.53450400-0.19552500$

C $\quad 6.88172200 \quad 2.73231300 \quad-1.02715200$

$\mathrm{H} \quad 5.87243000 \quad 3.11423100 \quad-1.19854100$

$\mathrm{H} \quad \begin{array}{llll}7.48744100 & 3.04178300 & -1.88219900\end{array}$

C $\quad-3.20240500-1.38219200-0.88116900$

C $\quad-3.21887300-2.76192800-0.65646500$

C $\quad-4.45994000-3.41321100-0.39635000$

C $\quad-5.60444100-2.62178900-0.29634300$

$\begin{array}{llll}\text { C } & -1.97406400 & -3.47304300 & -0.34762800\end{array}$

$\begin{array}{llll}\text { C } & -2.07942100 & -4.61885100 & 0.50534300\end{array}$

C $\quad-3.29945900-5.44896900 \quad 0.43762200$

C $\quad-4.48018700-4.85908500-0.10468800$

$\begin{array}{llll}\text { C } & -0.71349900 & -2.94110000 & -0.60938700\end{array}$

$\begin{array}{llll}\text { C } & 0.41934000 & -3.32177000 & 0.12614000\end{array}$

C $\quad-1.01500000-4.84827500 \quad 1.37949600$

C $\quad 1.73936600-2.77532100-0.11959200$

$\begin{array}{llll}\text { C } & 0.21470400 & -4.17746500 & 1.24663400\end{array}$ 


\begin{tabular}{|c|c|c|c|}
\hline ; & 2.72391100 & -2.85472000 & 0.90472300 \\
\hline C & 2.10865100 & -2.19763400 & -1.35041200 \\
\hline C & 4.29051700 & -1.58450700 & -0.46918000 \\
\hline C & 3.96410200 & -2.20260800 & 0.73116600 \\
\hline C & 3.36457600 & -1.65245500 & -1.53357700 \\
\hline C & 2.44814600 & -3.63688000 & 2.08400300 \\
\hline C & 1.26601900 & -4.31815100 & 2.22019400 \\
\hline $\mathrm{H}$ & -2.24176400 & -0.89784500 & -0.99879600 \\
\hline $\mathrm{H}$ & -6.54197300 & -3.05895400 & 0.02017800 \\
\hline $\mathrm{H}$ & -0.61895400 & -2.13701000 & -1.33112800 \\
\hline $\mathrm{H}$ & -1.12165200 & -5.54143700 & 2.20388800 \\
\hline 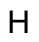 & 4.67079100 & -2.20438200 & 1.55418700 \\
\hline$\checkmark$ & -5.63290900 & -5.65370600 & -0.32834700 \\
\hline C & -5.69865600 & -6.91952800 & 0.27918300 \\
\hline C & -3.30475400 & -6.82902400 & 0.78199200 \\
\hline C & -4.54231100 & -7.49835500 & 0.84352000 \\
\hline $\mathrm{H}$ & 3.64559300 & -1.26477700 & -2.50620900 \\
\hline $\mathrm{H}$ & 1.40933100 & -2.21617300 & -2.18084300 \\
\hline & 7.44784600 & 3.38650500 & 0.24854800 \\
\hline & 6.82887200 & 3.09620100 & 1.10693600 \\
\hline & 8.45257700 & 2.99409200 & 0.45255900 \\
\hline & 7.49875200 & 4.91202700 & 0.13573800 \\
\hline & 8.14486500 & 5.22386500 & -0.69421600 \\
\hline & 6.50002500 & 5.32603600 & -0.05243700 \\
\hline & 7.88557800 & 5.36987200 & 1.05331300 \\
\hline & 6.65039300 & -3.67096800 & -2.22948900 \\
\hline & 5.73068700 & -3.26792000 & -2.67365300 \\
\hline & 7.48557300 & -3.27913200 & -2.82397300 \\
\hline C & 6.63035500 & -5.20004400 & -2.28758200 \\
\hline $\mathrm{H}$ & 7.55011300 & -5.62279100 & -1.86425400 \\
\hline & 5.78483300 & -5.60391000 & -1.71679800 \\
\hline & 6.54100900 & -5.55939000 & -3.31892600 \\
\hline & 3.67461500 & 3.62687700 & 2.86710100 \\
\hline & 1.39053600 & 5.23847800 & 3.06241400 \\
\hline & 3.42385800 & -3.70255500 & 3.05664400 \\
\hline & 1.04097200 & -5.15367900 & 3.29341000 \\
\hline $\mathrm{O}$ & -7.88409800 & 1.66729800 & -0.18720200 \\
\hline $\mathrm{O}$ & -7.96288400 & -1.12170600 & -0.07472900 \\
\hline C & 1.16208400 & 4.62918800 & 4.34113200 \\
\hline & 2.01961700 & 4.01957400 & 4.64321500 \\
\hline & 1.02076300 & 5.45092500 & 5.04721500 \\
\hline & 0.25737500 & 4.00833400 & 4.31642000 \\
\hline & 4.46999700 & 4.81948600 & 2.82980700 \\
\hline & 3.85768800 & 5.70419300 & 3.03444300 \\
\hline & 5.22972700 & 4.70392400 & 3.60656600 \\
\hline & 4.96116100 & 4.92684700 & 1.85411800 \\
\hline & -1.39320800 & 7.81449000 & 0.39387000 \\
\hline & -1.30964100 & 8.48483800 & 1.25915000 \\
\hline & -0.58950800 & 7.09361400 & 0.50813600 \\
\hline & -1.08996900 & 8.59720600 & -0.90129600 \\
\hline & -1.13513700 & 7.89462700 & -1.74383900 \\
\hline & -1.86097400 & 9.35159400 & -1.09212400 \\
\hline & 0.28632600 & 9.26754100 & -0.85378500 \\
\hline & 0.34340200 & 9.99268100 & -0.03205200 \\
\hline & 1.08101900 & 8.52782000 & -0.69686300 \\
\hline & 0.50391500 & 9.80084600 & -1.78628300 \\
\hline & -6.54444700 & 5.63505900 & -1.20528300 \\
\hline & -6.37135100 & 4.70438000 & -1.74914400 \\
\hline & -6.80590000 & 6.36649500 & -1.98040800 \\
\hline & -7.76876300 & 5.46412200 & -0.27758800 \\
\hline & -7.49268300 & 4.86928000 & 0.60232000 \\
\hline & -8.06683100 & 6.44086900 & 0.12050900 \\
\hline & -8.95063900 & 4.80583500 & -0.99482400 \\
\hline & -8.69926200 & 3.78304500 & -1.29518000 \\
\hline & -9.83274800 & 4.75822600 & -0.34490800 \\
\hline & -9.23008300 & 5.37007300 & -1.89357400 \\
\hline & & 8 & \\
\hline
\end{tabular}

H

$\mathrm{H}$

$\mathrm{C}$

\section{1-Benz TS}

$\mathrm{C}$
$\mathrm{C}$
$\mathrm{C}$
$\mathrm{C}$

$\begin{array}{lll}-7.09283900 & 8.03785500 & -0.94928100\end{array}$

$\begin{array}{lll}-6.68755500 & 8.72591700 & 0.61723100\end{array}$

$\begin{array}{lll}-5.93462500 & 9.35178900 & -0.84386400\end{array}$

$\begin{array}{lll}-3.82802400 & 9.42829500 & 0.65092800\end{array}$

$\begin{array}{lll}-2.89986000 & 9.68979200 & 1.15905800\end{array}$

$\begin{array}{lll}-3.91806200 & 10.08752000 & -0.22235200\end{array}$

$\begin{array}{lll}-4.65131500 & 9.69086100 & 1.32501300\end{array}$

$\begin{array}{lll}-6.98854800 & -7.71500900 & 0.30621900\end{array}$

$\begin{array}{lll}-7.83571200 & -7.15751400 & -0.09368000\end{array}$

$\begin{array}{lll}-6.90982900 & -8.65208700 & -0.26031500\end{array}$

$\begin{array}{lll}-7.24473200 & -7.99478600 & 1.33522100\end{array}$

$\begin{array}{lll}-4.66460100 & -8.88022500 & 1.45582500\end{array}$

$\begin{array}{lll}-5.43593400 & -8.88366200 & 2.23565300\end{array}$

$\begin{array}{lll}-4.96448700 & -9.63716100 & 0.71941500\end{array}$

$\begin{array}{lll}-3.73922900 & -9.22070300 & 1.92011300\end{array}$

$\begin{array}{lll}-8.45129100 & 1.65506700 & 1.12965600\end{array}$

$\begin{array}{lll}-8.77741500 & 0.64787100 & 1.40655300\end{array}$

$\begin{array}{lll}-7.72494200 & 2.02441900 & 1.86492800\end{array}$

$\begin{array}{lll}-9.30794600 & 2.33195800 & 1.09312100\end{array}$

$\begin{array}{lll}-8.84625000 & -1.15954800 & -1.20205800\end{array}$

$\begin{array}{lll}-9.14093600 & -0.14753600 & -1.50114800\end{array}$

$\begin{array}{lll}-8.37239700 & -1.67250200 & -2.04950500\end{array}$

$\begin{array}{lll}-9.72608400 & -1.72320100 & -0.88241900\end{array}$

$\begin{array}{lll}-6.71189900 & -5.25052200 & -1.32784200\end{array}$

$\begin{array}{lll}-7.71073600 & -5.23172100 & -0.87006600\end{array}$

$\begin{array}{lll}-6.53553100 & -4.24058300 & -1.69571900\end{array}$

$-6.72652500-6.17079200-2.56661300$

$\begin{array}{lll}-5.73105800 & -6.14068700 & -3.02848900\end{array}$

$\begin{array}{lll}-6.89585200 & -7.21342500 & -2.27586100\end{array}$

$\begin{array}{lll}-7.78835200 & -5.74154800 & -3.58372700\end{array}$

$\begin{array}{lll}-8.79420100 & -5.78814900 & -3.14720200\end{array}$

$\begin{array}{lll}-7.62111400 & -4.71013800 & -3.91870200\end{array}$

$\begin{array}{lll}-7.77841400 & -6.38645400 & -4.46978000\end{array}$

$\begin{array}{lll}-2.03010800 & -7.65795100 & 0.90427600\end{array}$

$\begin{array}{lll}-1.14626200 & -7.02711700 & 0.85459600\end{array}$

$\begin{array}{lll}-1.97057100 & -8.17499900 & 1.87160600\end{array}$

$\begin{array}{lll}-1.89454600 & -8.68218800 & -0.24285400\end{array}$

$\begin{array}{lll}-2.75090900 & -9.36497200 & -0.26105900\end{array}$

$\begin{array}{lll}-1.92033100 & -8.13553100 & -1.19467400\end{array}$

$\begin{array}{lll}-0.59614400 & -9.48789100 & -0.13827300\end{array}$

$\begin{array}{llll}0.28077600 & -8.82912900 & -0.15592900\end{array}$

$-0.56086300-10.05893900 \quad 0.79817900$

$\begin{array}{lll}-0.49862600 & -10.19817200 & -0.96728500\end{array}$

$0.82967300 \quad-4.47213400 \quad 4.53821000$

$\begin{array}{lll}0.62887000 & -5.24964200 & 5.27912200\end{array}$

$\begin{array}{lll}1.71741300 & -3.90051600 & 4.82716600\end{array}$

$\begin{array}{lll}-0.03586800 & -3.80096300 & 4.46819400\end{array}$

$\begin{array}{lll}4.14016600 & -4.94422300 & 3.08197400\end{array}$

$\begin{array}{lll}4.64044200 & -5.12203400 & 2.12069800\end{array}$

$\begin{array}{lll}4.89114200 & -4.84705500 & 3.86956900\end{array}$

$3.46771400 \quad-5.77866600 \quad 3.30741300$

$\begin{array}{llll}9.45606500 & 1.94728300 & -2.24154800\end{array}$

$\begin{array}{llll}9.70374000 & -2.27214400 & 0.02602400\end{array}$

$10.74470200 \quad 2.55681400 \quad-2.43618600$

$\begin{array}{llll}11.49967000 & 1.79108700 & -2.63247300\end{array}$

$10.62710700 \quad 3.21483600 \quad-3.29753300$

$\begin{array}{llll}11.03689600 & 3.12709900 & -1.54971600\end{array}$

$10.97462600-2.94043600-0.06821700$

$\begin{array}{lll}11.16273000 & -3.34955100 & 0.92474600\end{array}$

$10.93761600 \quad-3.73748300 \quad-0.81630600$

$\begin{array}{lll}11.75127100 & -2.22273500 & -0.34538100\end{array}$

$\begin{array}{llll}-4.09460800 & 0.84468500 & 0.24528300\end{array}$

$\begin{array}{lll}-5.31780200 & 1.56311800 & 0.12665400\end{array}$

$\begin{array}{lll}-4.11413000 & -0.58013600 & 0.54303800\end{array}$

$\begin{array}{lll}-2.95575900 & -1.36713800 & 0.60772300\end{array}$ 
C$$
\mathrm{H}
$$

$\begin{array}{lll}3.28180000 & 4.82229500 & -0.95663400\end{array}$ $\begin{array}{lll}2.11802100 & 5.54789700 & -0.95957600\end{array}$ $\begin{array}{lll}-1.96991000 & 1.07023300 & 0.22104900\end{array}$ $\begin{array}{lll}-6.24551900 & 3.44124900 & -0.16250400\end{array}$ $\begin{array}{lll}-0.53104000 & 1.79120400 & -0.87859300\end{array}$ $\begin{array}{llll}-0.27903300 & 6.69946400 & -0.86095600\end{array}$ $\begin{array}{lll}5.33988000 & 3.22035600 & -0.68798600\end{array}$ $\begin{array}{llll}-5.18617900 & 5.82602700 & -1.25861400\end{array}$ $\begin{array}{llll}-5.13980200 & 7.23060600 & -1.22022200\end{array}$ $\begin{array}{llll}-2.93032700 & 7.16842800 & -0.16824900\end{array}$ $\begin{array}{llll}-4.07752100 & 7.88437100 & -0.54902400\end{array}$ $\begin{array}{lll}3.15977900 & -0.45189600 & -0.96964600\end{array}$ $\begin{array}{lll}1.15854800 & 0.73718300 & -1.27329400\end{array}$ $\begin{array}{llll}9.96365900 & -1.43518400 & 1.99340800\end{array}$ $\begin{array}{llll}10.16469900 & 1.43466500 & 0.88572200\end{array}$ $\begin{array}{lll}11.16725200 & -2.85811700 & 0.12962200\end{array}$ $11.42205800 \quad-3.36707100 \quad-0.80025500$ $\begin{array}{lll}11.91814800 & -2.10390100 & 0.37902200\end{array}$ $\begin{array}{lll}11.09214800 & -3.57269300 & 0.95421400\end{array}$ $\begin{array}{llll}10.13565500 & 1.87754100 & 3.50002900\end{array}$ $\begin{array}{lll}10.44127300 & 2.84006100 & 3.07944900\end{array}$ $\begin{array}{llll}9.82920100 & 1.99081000 & 4.54029300\end{array}$ $10.96205000 \quad 1.16701500 \quad 3.41456200$ $\begin{array}{llll}4.51143300 & 5.44085200 & -0.87192500\end{array}$ $\begin{array}{lll}2.14538800 & 6.92885400 & -0.94803400\end{array}$ $\begin{array}{lll}4.89758000 & 6.19619600 & -2.02875000\end{array}$ $\begin{array}{llll}4.21100900 & 7.03078800 & -2.19857300\end{array}$ $\begin{array}{lll}5.90170900 & 6.57250600 & -1.82022500\end{array}$ $\begin{array}{lll}4.92514200 & 5.55268900 & -2.91715400\end{array}$ $\begin{array}{lll}2.43149400 & 7.48249900 & 0.34378100\end{array}$ $\begin{array}{lll}3.41988200 & 7.16543200 & 0.69461500\end{array}$ $\begin{array}{lll}2.41142500 & 8.56837200 & 0.22283300\end{array}$ $\begin{array}{lll}1.66656800 & 7.18121600 & 1.07161200\end{array}$ $\begin{array}{lll}-6.37244800 & 5.13782800 & -1.92256900\end{array}$ $\begin{array}{lll}-6.12519000 & 4.09756500 & -2.14329300\end{array}$ $\begin{array}{lll}-6.54272300 & 5.59697100 & -2.90508200\end{array}$ $\begin{array}{lll}-1.85433700 & 7.88862200 & 0.64890100\end{array}$ $\begin{array}{lll}-0.98319700 & 8.15932900 & 0.04106500\end{array}$ $\begin{array}{lll}-2.26053800 & 8.84569100 & 0.98020800\end{array}$ $\begin{array}{llll}-7.73005900 & 1.59435300 & 0.20536100\end{array}$ $\begin{array}{lll}-7.77673700 & -1.15526100 & 0.76046600\end{array}$ $\begin{array}{lll}-8.46500100 & 1.77389200 & 1.42283500\end{array}$ $\begin{array}{lll}-8.81116000 & 0.81350900 & 1.81832300\end{array}$ $\begin{array}{lll}-7.84736900 & 2.28118900 & 2.17477100\end{array}$ $\begin{array}{lll}-9.31973800 & 2.40452900 & 1.16752500\end{array}$ $\begin{array}{lll}-8.58434200 & -1.29580800 & -0.41597800\end{array}$ $-8.03335000-1.83156400-1.19935200$ $\begin{array}{lll}-9.45473200 & -1.88360400 & -0.11533300\end{array}$ $\begin{array}{lll}-8.90291000 & -0.31868500 & -0.79311300\end{array}$ $\begin{array}{lll}-6.22047500 & -4.82226400 & 2.66248800\end{array}$ $\begin{array}{lll}-5.86532000 & -3.80471500 & 2.83303700\end{array}$ $\begin{array}{lll}-6.20516700 & -5.29410500 & 3.65345700\end{array}$ $\begin{array}{lll}-2.35700900 & -7.89077600 & -0.54189300\end{array}$ $\begin{array}{lll}-1.34303400 & -7.58237900 & -0.27598500\end{array}$ $\begin{array}{lll}-2.41906200 & -8.93111200 & -0.21714100\end{array}$ $\begin{array}{lll}1.10944500 & -6.16909300 & -2.53875800\end{array}$ $\begin{array}{lll}3.58977600 & -4.82557800 & -2.63762400\end{array}$ $\begin{array}{lll}1.87286700 & -7.35579800 & -2.28234900\end{array}$ $\begin{array}{lll}1.50745700 & -8.10759400 & -2.98575100\end{array}$ $\begin{array}{lll}2.94162900 & -7.18076100 & -2.44291500\end{array}$ $\begin{array}{lll}1.70832300 & -7.70563900 & -1.25517100\end{array}$ $3.28902700 \quad-4.61416500-4.02431100$ $\begin{array}{lll}4.18518900 & -4.90989300 & -4.57432900\end{array}$ $\begin{array}{lll}2.43577700 & -5.22216300 & -4.34071000\end{array}$ $\begin{array}{lll}3.07520100 & -3.55476400 & -4.21380800\end{array}$ $\begin{array}{lll}7.36748100 & -3.54065400 & -1.43373600\end{array}$ $\begin{array}{lll}6.75977600 & -2.94785500 & -2.12793800\end{array}$ 


1-Se GS
C
C
C
C
C
C
C
C
C

$8.40722100 \quad-3.28124000 \quad-1.65184600$

$\begin{array}{lll}7.11545300 & -5.03141800 & -1.67446000\end{array}$

$\begin{array}{lll}7.66897200 & -5.64718400 & -0.95408100\end{array}$

$6.04942700 \quad-5.26928800-1.57970700$

$\begin{array}{lll}7.43424100 & -5.32893800 & -2.68047100\end{array}$

$\begin{array}{lll}5.82083600 & 3.06028000 & 2.07791800\end{array}$

$\begin{array}{lll}4.81812600 & 2.72706200 & 1.79324100\end{array}$

$\begin{array}{lll}6.08903700 & 2.51102000 & 2.98945400\end{array}$

$\begin{array}{lll}5.80302800 & 4.56807300 & 2.34410700\end{array}$

$\begin{array}{llll}6.79831400 & 4.93220200 & 2.63022300\end{array}$

$\begin{array}{lll}5.48830900 & 5.11324300 & 1.44643400\end{array}$

$\begin{array}{llll}5.11049000 & 4.81889900 & 3.15596400\end{array}$

$\begin{array}{lll}-6.30484300 & -7.75316600 & 2.53550000\end{array}$

$\begin{array}{lll}-6.70740900 & -7.17001100 & 3.36315400\end{array}$

$\begin{array}{lll}-7.16069300 & -8.09848200 & 1.93971800\end{array}$

$\begin{array}{lll}-5.84025100 & -8.64426000 & 2.96938300\end{array}$

$\begin{array}{lll}-4.64242900 & -9.18538300 & 0.74575400\end{array}$

$\begin{array}{lll}-4.17273100 & -9.72576400 & 1.58016900\end{array}$

$\begin{array}{lll}-5.70644300 & -9.43592700 & 0.76453700\end{array}$

$\begin{array}{lll}-4.24470500 & -9.60376100 & -0.17557900\end{array}$

$\begin{array}{lll}-2.53644300 & -7.84393600 & -2.08063300\end{array}$

$\begin{array}{lll}-2.58883000 & -6.79960500 & -2.40905000\end{array}$

$\begin{array}{lll}-1.64179800 & -8.27259100 & -2.55322100\end{array}$

$\begin{array}{llll}-3.78347400 & -8.57013500 & -2.59512600\end{array}$

$\begin{array}{lll}-3.73951900 & -9.64601800 & -2.38661000\end{array}$

$\begin{array}{lll}-4.69200300 & -8.17152100 & -2.12970300\end{array}$

$\begin{array}{lll}-3.88248200 & -8.44883500 & -3.67975100\end{array}$

$\begin{array}{lll}-7.68593100 & -4.77231200 & 2.17503600\end{array}$

$\begin{array}{lll}-7.71453200 & -4.51869300 & 1.10779000\end{array}$

$\begin{array}{lll}-8.13312000 & -5.77054700 & 2.24773800\end{array}$

$\begin{array}{lll}-8.52409700 & -3.76168300 & 2.96302600\end{array}$

$\begin{array}{lll}-8.47564600 & -3.96337900 & 4.04060100\end{array}$

$\begin{array}{lll}-8.16513800 & -2.74188400 & 2.78648900\end{array}$

$\begin{array}{lll}-9.57823900 & -3.80040600 & 2.66367200\end{array}$

$\begin{array}{lll}-6.23444100 & 8.07094900 & -1.85463600\end{array}$

$\begin{array}{lll}-6.85392700 & 7.49986000 & -2.54550400\end{array}$

$\begin{array}{llll}-6.90813500 & 8.50468300 & -1.10400700\end{array}$

$\begin{array}{llll}-5.81001500 & 8.90319700 & -2.42579600\end{array}$

$\begin{array}{llll}-4.16924100 & 9.37448000 & -0.26312700\end{array}$

$\begin{array}{lll}-3.30539500 & 9.92381200 & -0.65532800\end{array}$

$\begin{array}{lll}-5.06129200 & 9.82941100 & -0.69113500\end{array}$

$\begin{array}{lll}-4.20814800 & 9.56610000 & 0.81667600\end{array}$

$\begin{array}{llll}-7.69706800 & 5.19318900 & -1.12979400\end{array}$

$\begin{array}{llll}-7.51632800 & 4.92244600 & -0.08161300\end{array}$

$\begin{array}{llll}-8.06482100 & 6.22539100 & -1.10269500\end{array}$

$\begin{array}{lll}-8.76489400 & 4.26851500 & -1.72119600\end{array}$

$\begin{array}{lll}-8.94064600 & 4.49464500 & -2.78054500\end{array}$

$\begin{array}{llll}-8.45592900 & 3.22062100 & -1.63988200\end{array}$

$\begin{array}{llll}-9.72070800 & 4.37779300 & -1.19481400\end{array}$

$\begin{array}{lll}-1.39377400 & 7.15323700 & 1.92603900\end{array}$

$\begin{array}{lll}-0.88036100 & 6.22101600 & 1.67486300\end{array}$

$\begin{array}{lll}-0.65146000 & 7.79080800 & 2.42631800\end{array}$

$\begin{array}{lll}-2.54784800 & 6.85748300 & 2.88983900\end{array}$

$\begin{array}{lll}-3.07115300 & 7.77893300 & 3.17604300\end{array}$

$\begin{array}{lll}-3.28122200 & 6.18768400 & 2.42841800\end{array}$

$\begin{array}{lll}-2.18419300 & 6.37951000 & 3.80637800\end{array}$

$\begin{array}{lll}3.67264900 & 0.46822700 & 0.19341600\end{array}$

$\begin{array}{llll}4.95428100 & 1.06887000 & 0.33295800\end{array}$

$\begin{array}{llll}3.56902900 & -0.95865300 & -0.05585000\end{array}$

$\begin{array}{llll}2.34319100 & -1.61828300 & -0.17642800\end{array}$

$\begin{array}{lll}2.23833300 & -2.98573100 & -0.41397700\end{array}$

$\begin{array}{lll}3.41385600 & -3.78597700 & -0.50658400\end{array}$

$\begin{array}{llll}4.64178600 & -3.13712100 & -0.33389300\end{array}$

$\begin{array}{llll}0.93167600 & -3.62880600 & -0.46874700\end{array}$

$\begin{array}{llll}0.84282300 & -5.02218600 & -0.16747000\end{array}$
$2.07971200 \quad-5.83307900 \quad-0.08809900$

$\begin{array}{llll}3.28304600 & -5.25734300 & -0.60064600\end{array}$

$-0.21934200 \quad-2.89441900-0.74763800$

$-1.50716000-3.42681400-0.62019800$

$\begin{array}{llll}-0.44197700 & -5.56785700 & -0.09415200\end{array}$

$-2.71253500 \quad-2.63205100 \quad-0.77889500$

$\begin{array}{lll}4.74822800 & -1.74924800 & -0.14043100\end{array}$

$\begin{array}{lll}-1.60678700 & -4.79573100 & -0.23596100\end{array}$

$\begin{array}{lll}-3.94659600 & -3.17415700 & -0.32784900\end{array}$

$\begin{array}{lll}-5.07908400 & -2.34224900 & -0.23250500\end{array}$

$-2.73307600-1.32146300-1.30685200$

$\begin{array}{lll}6.13106100 & 0.23894700 & 0.23781200\end{array}$

$\begin{array}{lll}-4.02014700 & -4.56286700 & 0.05480000\end{array}$

$\begin{array}{lll}-3.88000500 & -0.55053700 & -1.28917800\end{array}$

$\begin{array}{llll}6.03245900 & -1.11015900 & 0.01781900\end{array}$

$\begin{array}{lll}-2.90543800 & -5.35940600 & 0.03873000\end{array}$

$\begin{array}{lll}-5.06758200 & -1.02315000 & -0.67746300\end{array}$

$\begin{array}{lll}1.43135500 & -1.05790900 & -0.02079400\end{array}$

$\begin{array}{llll}5.55980200 & -3.69896800 & -0.29702200\end{array}$

$\begin{array}{lll}-0.11181300 & -1.85606600 & -1.03591600\end{array}$

$\begin{array}{lll}-0.58555600 & -6.61619100 & 0.10359800\end{array}$

$\begin{array}{lll}-5.96507500 & -2.75348200 & 0.23123400\end{array}$

$\begin{array}{lll}-1.83142200 & -0.90204700 & -1.74199200\end{array}$

$\begin{array}{llll}-3.85768400 & 0.44939700 & -1.70831600\end{array}$

$\begin{array}{llll}4.30548800 & -6.11129400 & -1.09955800\end{array}$

$\begin{array}{llll}4.19738300 & -7.49732700 & -0.89317700\end{array}$

$\begin{array}{llll}3.18337300 & -8.00398600 & -0.04388500\end{array}$

$\begin{array}{llll}2.12483700 & -7.18019500 & 0.37546600\end{array}$

$\begin{array}{lll}2.55800900 & 1.30942100 & 0.24925900\end{array}$

$\begin{array}{lll}2.65456600 & 2.68112600 & 0.46508300\end{array}$

$\begin{array}{llll}3.93355000 & 3.29170900 & 0.60971100\end{array}$

$\begin{array}{lll}5.05395800 & 2.45929900 & 0.51128700\end{array}$

$\begin{array}{lll}1.46430300 & 3.52140100 & 0.42959000\end{array}$

$\begin{array}{lll}1.61384600 & 4.90012100 & 0.08439700\end{array}$

$\begin{array}{lll}2.96399400 & 5.50894300 & 0.07451800\end{array}$

$\begin{array}{llll}4.02779600 & 4.76695900 & 0.67462600\end{array}$

$\begin{array}{llll}0.19847400 & 2.98422200 & 0.65631500\end{array}$

$\begin{array}{lll}-0.98094400 & 3.70119600 & 0.42216800\end{array}$

$\begin{array}{llll}0.43638800 & 5.62969100 & -0.09878900\end{array}$

$\begin{array}{lll}-2.30464900 & 3.10881800 & 0.51632800\end{array}$

$\begin{array}{llll}-0.83924800 & 5.04715500 & -0.02134600\end{array}$

$\begin{array}{lll}-3.40938000 & 3.81164500 & -0.04071000\end{array}$

$\begin{array}{lll}-2.56064800 & 1.84933100 & 1.10284900\end{array}$

$\begin{array}{lll}-4.87692000 & 1.89228200 & 0.35442800\end{array}$

$\begin{array}{lll}-4.65955500 & 3.16927200 & -0.15525900\end{array}$

$\begin{array}{lll}-3.81322300 & 1.26874500 & 1.05070800\end{array}$

$\begin{array}{lll}-3.22966600 & 5.16630900 & -0.50263000\end{array}$

$-2.00751800 \quad 5.77855300 \quad-0.43940100$

$\begin{array}{lll}1.57923300 & 0.89348800 & 0.05131900\end{array}$

$\begin{array}{lll}6.04815300 & 2.87391500 & 0.51953300\end{array}$

$\begin{array}{lll}0.12778000 & 1.95418400 & 0.98387700\end{array}$

$\begin{array}{llll}0.46493500 & 6.68151900 & -0.32633500\end{array}$

$\begin{array}{lll}-5.45041600 & 3.68357100 & -0.68896600\end{array}$

$\begin{array}{lll}5.13657600 & 5.46235500 & 1.23004000\end{array}$

$\begin{array}{lll}5.25964100 & 6.84341500 & 0.99983800\end{array}$

$\begin{array}{lll}3.24856800 & 6.82226400 & -0.40075900\end{array}$

$\begin{array}{llll}4.39474400 & 7.48086000 & 0.07720100\end{array}$

$\begin{array}{lll}-3.97392000 & 0.29803600 & 1.50746900\end{array}$

$\begin{array}{lll}-1.76090200 & 1.31922200 & 1.61028800\end{array}$

$\begin{array}{lll}-4.32460000 & 5.82339300 & -1.02406200\end{array}$

$\begin{array}{llll}-1.85309900 & 7.09632400 & -0.81698000\end{array}$

$\begin{array}{llll}-4.88862400 & 6.80495000 & -0.14537400\end{array}$

$\begin{array}{lll}-4.16219500 & 7.59449600 & 0.07429100\end{array}$

$\begin{array}{llll}-5.74892400 & 7.22680800 & -0.67073000\end{array}$

$\begin{array}{lll}-5.22499100 & 6.33990000 & 0.79091400\end{array}$

$\begin{array}{lll}-1.85199100 & 7.29918400 & -2.23690500\end{array}$

$\begin{array}{llll}-2.80519200 & 6.98584100 & -2.67562800\end{array}$ 


\begin{tabular}{|c|c|c|c|}
\hline $\mathrm{H}$ & -1.70594700 & 8.37110700 & -2.39135400 \\
\hline $\mathrm{H}$ & -1.02646100 & 6.74770000 & -2.70551000 \\
\hline C & 6.13904000 & 4.75769400 & 2.13463700 \\
\hline $\mathrm{H}$ & 5.75135200 & 3.78019800 & 2.42838700 \\
\hline $\mathrm{H}$ & 6.22883100 & 5.31690400 & 3.07513300 \\
\hline C & 2.42934100 & 7.49461200 & -1.50498200 \\
\hline $\mathrm{H}$ & 1.50686200 & 7.94855400 & -1.12359500 \\
\hline 11 & 3.00456000 & 8.33716400 & -1.89179700 \\
\hline$\checkmark$ & 7.35662200 & 0.84816000 & 0.41570600 \\
\hline 0 & 7.15697400 & -1.90028700 & -0.10563900 \\
\hline$\checkmark$ & 8.15718400 & 0.94413200 & -0.76953100 \\
\hline $\mathrm{H}$ & 8.42215600 & -0.04941700 & -1.14556400 \\
\hline H & 7.62564300 & 1.50401200 & -1.54956100 \\
\hline $\mathrm{H}$ & 9.05891800 & 1.48888200 & -0.48039400 \\
\hline & 7.88647600 & -2.10410500 & 1.11155000 \\
\hline & 7.24666300 & -2.57159600 & 1.87081400 \\
\hline H & 8.70768300 & -2.77972800 & 0.86104600 \\
\hline 11 & 8.28190800 & -1.15818300 & 1.49539900 \\
\hline C & 5.46320100 & -5.56207900 & -1.92280600 \\
\hline $\mathrm{H}$ & 5.24984100 & -4.53511800 & -2.22573100 \\
\hline 11 & 5.53096100 & -6.12209300 & -2.86470100 \\
\hline C & 1.14294500 & -7.72517400 & 1.41549900 \\
\hline 1 & 0.18556000 & -8.02538800 & 0.97349000 \\
\hline $\mathrm{H}$ & 1.55527400 & -8.65014400 & 1.82255400 \\
\hline 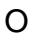 & -2.96294100 & -6.69568200 & 0.37570100 \\
\hline $\mathrm{O}$ & -5.26660300 & -5.05987100 & 0.37785100 \\
\hline C & -3.68278500 & -7.51355000 & -0.55644300 \\
\hline $\mathrm{H}$ & -3.59756100 & -8.53845300 & -0.18765000 \\
\hline $\mathrm{H}$ & -4.73657700 & -7.22120700 & -0.60816000 \\
\hline $\mathrm{H}$ & -3.23445300 & -7.44561700 & -1.55576500 \\
\hline C & -5.41719200 & -5.47410400 & 1.74237500 \\
\hline $\mathrm{H}$ & -6.45780900 & -5.78949100 & 1.84787000 \\
\hline $\mathrm{H}$ & -4.74614200 & -6.30574200 & 1.97951500 \\
\hline $\mathrm{H}$ & -5.21741200 & -4.63663400 & 2.42282700 \\
\hline C & 5.17906700 & -8.46503500 & -1.52975700 \\
\hline $\mathrm{H}$ & 5.75547600 & -8.00768000 & -2.33 \\
\hline $\mathrm{H}$ & 5.89970800 & -8.86093200 & -0.80223000 \\
\hline$H$ & 4.65707700 & -9.32353900 & -1.96543100 \\
\hline C & 3.23289000 & -9.45580900 & 0.40412400 \\
\hline $\mathrm{H}$ & 2.28542300 & -9.97617500 & 0.22408700 \\
\hline $\mathrm{H}$ & 4.01204000 & -10.02365500 & -0.10244500 \\
\hline $\mathrm{H}$ & 3.44079800 & -9.52975200 & 1.47946000 \\
\hline C & 0.89575200 & -6.79924600 & 2.62608600 \\
\hline $\mathrm{H}$ & 0.39808900 & -5.87669200 & 2.31562900 \\
\hline $\mathrm{H}$ & 0.19792200 & -7.31373500 & 3.30030300 \\
\hline C & 2.18431400 & -6.45674500 & 3.38193700 \\
\hline $\mathrm{H}$ & 2.69617000 & -7.36500600 & 3.72581500 \\
\hline $\mathrm{H}$ & 2.88052500 & -5.90491800 & 2.74123200 \\
\hline $\mathrm{H}$ & 1.97303300 & -5.83789200 & 4.26133900 \\
\hline C & 6.84235800 & -5.61511400 & -1.22815000 \\
\hline $\mathrm{H}$ & 6.75102000 & -5.26335700 & -0.19240100 \\
\hline $\mathrm{H}$ & 7.17393800 & -6.65717800 & -1.15188300 \\
\hline C & 7.89459500 & -4.77871000 & -1.96183200 \\
\hline $\mathrm{H}$ & 7.97296900 & -5.07775900 & -3.01472300 \\
\hline $\mathrm{H}$ & 7.63646000 & -3.71451600 & -1.92507400 \\
\hline $\mathrm{H}$ & 8.88523400 & -4.89803900 & -1.50696400 \\
\hline C & 6.33611700 & 7.66024100 & 1.69181700 \\
\hline $\mathrm{H}$ & 6.76470100 & 7.14146600 & 2.54910800 \\
\hline $\mathrm{H}$ & 7.16603300 & 7.90733400 & 1.01690500 \\
\hline $\mathrm{H}$ & 5.93207400 & 8.60657800 & 2.06616100 \\
\hline C & 4.70155400 & 8.89572700 & -0.38679300 \\
\hline $\mathrm{H}$ & 3.84250300 & 9.56568900 & -0.26742700 \\
\hline U & 5.53415000 & 9.34185300 & 0.15519800 \\
\hline $\mathrm{H}$ & 4.97676300 & 8.91114700 & -1.44925200 \\
\hline C & 7.55337500 & 4.58752800 & 1.53557500 \\
\hline & 7.47768800 & 4.24642300 & 0.49497400 \\
\hline $\mathrm{H}$ & 8.05018400 & 5.56340200 & 1.48565700 \\
\hline
\end{tabular}

$\begin{array}{lrrr}\mathrm{C} & 8.40821700 & 3.60043700 & 2.33565400 \\ \mathrm{H} & 8.45785000 & 3.88769000 & 3.39354300 \\ \mathrm{H} & 7.99098300 & 2.58920500 & 2.27317300 \\ \mathrm{H} & 9.43488600 & 3.56227900 & 1.95227600 \\ \mathrm{C} & 2.11576200 & 6.59836900 & -2.72298300 \\ \mathrm{H} & 1.46201700 & 5.76864600 & -2.43959700 \\ \mathrm{H} & 1.55222900 & 7.20473300 & -3.44564200 \\ \mathrm{C} & 3.37891000 & 6.04506800 & -3.39173400 \\ \mathrm{H} & 4.04942600 & 6.85597800 & -3.70463500 \\ \mathrm{H} & 3.93517600 & 5.39996500 & -2.70347000 \\ \mathrm{H} & 3.12894300 & 5.45446600 & -4.28027300 \\ \mathrm{C} & -7.53232100 & -0.57877800 & -0.72593900 \\ \mathrm{C} & -6.25298500 & -0.15163500 & -0.46018700 \\ \mathrm{C} & -6.16866600 & 1.19901400 & 0.10554600 \\ \mathrm{C} & -7.37978500 & 1.80326600 & 0.34129100 \\ \mathrm{Se} & -8.79929100 & 0.70350600 & -0.21394100 \\ \mathrm{C} & -8.00329800 & -1.88555700 & -1.29630300 \\ \mathrm{H} & -7.15200900 & -2.39936800 & -1.75509300 \\ \mathrm{H} & -8.72871400 & -1.70819900 & -2.10218800 \\ \mathrm{C} & -8.65202000 & -2.80659300 & -0.23742000 \\ \mathrm{H} & -9.63524100 & -2.40037500 & 0.03499400 \\ \mathrm{H} & -8.05349200 & -2.77585000 & 0.68178800 \\ \mathrm{C} & -8.77450200 & -4.25357200 & -0.72023900 \\ \mathrm{H} & -9.30941900 & -4.87285400 & 0.00924100 \\ \mathrm{H} & -9.31864700 & -4.31180800 & -1.67115400 \\ \mathrm{H} & -7.77968300 & -4.69108500 & -0.86823500 \\ \mathrm{C} & -7.66864400 & 3.13059600 & 0.98655400 \\ \mathrm{H} & -8.06464100 & 3.83826900 & 0.24255200 \\ \mathrm{H} & -6.72573600 & 3.55801400 & 1.34392900 \\ \mathrm{C} & -8.65219700 & 3.05282000 & 2.17031300 \\ \mathrm{H} & -9.61937000 & 2.66166600 & 1.82670800 \\ \mathrm{H} & -8.27015300 & 2.32739400 & 2.89968900 \\ \mathrm{C} & -8.85734600 & 4.41624100 & 2.83527900 \\ \mathrm{H} & -9.56201400 & 4.35096500 & 3.67167200 \\ \mathrm{H} & -7.91071700 & 4.81140500 & 3.22432800 \\ \mathrm{H} & -9.25288700 & 5.14812100 & 2.11984000\end{array}$

\section{1-Se GS conf 2}

$\begin{array}{lrrr}\text { C } & -3.78233800 & -0.54235300 & -0.60634600 \\ \text { C } & -4.99334600 & -1.18943200 & -0.21675100 \\ \text { C } & -3.73914300 & 0.91783000 & -0.64194900 \\ \text { C } & -2.56927200 & 1.65583800 & -0.87333900 \\ \text { C } & -2.49136400 & 3.04202200 & -0.70294200 \\ \text { C } & -3.66160700 & 3.77857100 & -0.33685700 \\ \text { C } & -4.82623700 & 3.04634100 & -0.11549300 \\ \text { C } & -1.17955800 & 3.67866300 & -0.55149700 \\ \text { C } & -1.13819800 & 4.85316500 & 0.26222100 \\ \text { C } & -2.29216200 & 5.77563000 & 0.22346700 \\ \text { C } & -3.57197500 & 5.24408000 & -0.12652000 \\ \text { C } & 0.02041600 & 3.06620400 & -0.90669200 \\ \text { C } & 1.22844500 & 3.39253000 & -0.27056000 \\ \text { C } & -0.00287900 & 5.02829600 & 1.05458000 \\ \text { C } & 2.48944500 & 2.76326300 & -0.60756200 \\ \text { C } & -4.89941600 & 1.65313200 & -0.26532600 \\ \text { C } & 1.16438800 & 4.26828000 & 0.85188700 \\ \text { C } & 3.53022200 & 2.74822600 & 0.36135900 \\ \text { C } & 4.68858600 & 1.97807400 & 0.12262700 \\ \text { C } & 2.74871800 & 2.19441500 & -1.87090700 \\ \text { C } & -6.17055000 & -0.40570100 & 0.05871500 \\ \text { C } & 3.39579100 & 3.55548900 & 1.54750800 \\ \text { C } & 3.94673000 & 1.55435300 & -2.12807400 \\ \text { C } & -6.12646300 & 0.95952500 & 0.03414000 \\ \text { C } & 2.28866800 & 4.33884300 & 1.74831800 \\ \text { C } & 4.88831100 & 1.34159400 & -1.09580000 \\ \text { H } & -1.65128400 & 1.11798600 & -1.07273900 \\ \text { H } & -5.71190200 & 3.53107400 & 0.25534000 \\ \text { H } & 0.01103100 & 2.24236700 & -1.61266200\end{array}$




\begin{tabular}{|c|c|c|c|}
\hline $\mathrm{H}$ & -0.00372700 & 5.74872600 & 1.86300800 \\
\hline 11 & 5.42785400 & 1.89242500 & 0.91107300 \\
\hline 11 & 2.01331300 & 2.30106500 & -2.66306200 \\
\hline $\mathrm{H}$ & 4.15046700 & 1.16311900 & -3.11988600 \\
\hline 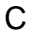 & -4.67638500 & 6.12380000 & -0.30492000 \\
\hline C & -4.52243600 & 7.48570700 & 0.01921000 \\
\hline C & -3.26575200 & 7.99918100 & 0.39974000 \\
\hline C & -2.12396800 & 7.17674800 & 0.39254300 \\
\hline C & -2.65359600 & -1.35426800 & -0.78876700 \\
\hline C & -2.67074000 & -2.74002200 & -0.59867600 \\
\hline$\checkmark$ & -3.89925100 & -3.38524300 & -0.26996700 \\
\hline$\checkmark$ & -5.01714500 & -2.58134100 & -0.04766400 \\
\hline C & -1.41992700 & -3.48456000 & -0.41807800 \\
\hline C & -1.48022400 & -4.68887700 & 0.35566200 \\
\hline C & -2.72038600 & -5.49046800 & 0.33880900 \\
\hline $\mathrm{O}$ & -3.92986200 & -4.84423100 & -0.05513700 \\
\hline O & -0.17316500 & -2.95260000 & -0.73707300 \\
\hline $\mathrm{C}$ & 1.00943300 & -3.41416900 & -0.13913500 \\
\hline $\mathrm{C}$ & -0.34901700 & -5.01107100 & 1.10884000 \\
\hline C & 2.31287100 & -2.86788000 & -0.45658700 \\
\hline C & 0.88503500 & -4.36439800 & 0.91495500 \\
\hline C & 3.39184500 & -3.07285900 & 0.44740400 \\
\hline C & 2.57119500 & -2.16088500 & -1.64863900 \\
\hline C & 4.83331500 & -1.64976000 & -0.91269000 \\
\hline$\checkmark$ & 4.62315600 & -2.41552900 & 0.22699800 \\
\hline C & 3.81007400 & -1.60586500 & -1.88932700 \\
\hline$C$ & 3.21113300 & -3.97177900 & 1.55928800 \\
\hline$C$ & 2.02480100 & -4.63113900 & 1.75374800 \\
\hline $\mathrm{H}$ & -1.69971500 & -0.87572700 & -0.96919800 \\
\hline $\mathrm{H}$ & -5.93148800 & -3.01465800 & 0.33429600 \\
\hline $\mathrm{H}$ & -0.11870900 & -2.09458100 & -1.39766400 \\
\hline $\mathrm{H}$ & -0.40250800 & -5.76752300 & 1.88086000 \\
\hline $\mathrm{H}$ & 5.40337400 & -2.51096300 & 0.97381100 \\
\hline C & -5.11793600 & -5.60227100 & -0.21974000 \\
\hline $\mathrm{C}$ & -5.15990000 & -6.90068700 & 0.31708900 \\
\hline C & -2.72793800 & -6.89075700 & 0.59073000 \\
\hline C & -3.97066500 & -7.53788600 & 0.72988500 \\
\hline $\mathrm{H}$ & 4.00190000 & -1.10380600 & -2.83032300 \\
\hline $\mathrm{H}$ & 1.79662900 & -2.08716000 & -2.40577500 \\
\hline $\mathrm{O}$ & 4.43359500 & 09500 & 2.45717100 \\
\hline 0 & 2.21134300 & 5.20956500 & 2.81369600 \\
\hline $\mathrm{O}$ & 4.28170900 & -4.17202000 & 2.40779600 \\
\hline O & 1.88367900 & -5.57214300 & 2.75143500 \\
\hline O & -7.25297500 & 1.71788900 & 0.27328700 \\
\hline $\mathrm{O}$ & -7.33190700 & -1.07138600 & 0.39479900 \\
\hline C & 2.02358500 & 4.57336500 & 4.08576100 \\
\hline $\mathrm{H}$ & 2.87456900 & 3.92975100 & 4.33015300 \\
\hline $\mathrm{H}$ & 1.94240600 & 5.37953900 & 4.81883800 \\
\hline $\mathrm{H}$ & 1.09891900 & 3.98193900 & 4.08901500 \\
\hline C & 5.30056100 & 4.68614400 & 2.34762400 \\
\hline $\mathrm{H}$ & 4.75257300 & 5.61417500 & 2.54497800 \\
\hline $\mathrm{H}$ & 6.08211400 & 4.54909200 & 3.09893400 \\
\hline $\mathrm{H}$ & 5.75857200 & 4.73106700 & 1.35029300 \\
\hline C & -0.75890700 & 7.85828900 & 0.36193100 \\
\hline $\mathrm{H}$ & -0.62531300 & 8.52832400 & 1.22098700 \\
\hline $\mathrm{H}$ & 0.05060200 & 7.13806400 & 0.42898100 \\
\hline C & -0.53188400 & 8.64128900 & -0.94846400 \\
\hline $\mathrm{H}$ & -0.63177000 & 7.94005000 & -1.78740800 \\
\hline $\mathrm{H}$ & -1.30966700 & 9.39948900 & -1.09110100 \\
\hline C & 0.84789700 & 9.30506100 & -0.98501800 \\
\hline $\mathrm{H}$ & 0.96092200 & 10.02555400 & -0.16496400 \\
\hline $\mathrm{H}$ & 1.64726300 & 8.56080000 & -0.88285400 \\
\hline $\mathrm{H}$ & 1.00837200 & 9.84231600 & -1.92680100 \\
\hline C & -6.00036700 & 5.66720300 & -0.90490100 \\
\hline $\mathrm{H}$ & -5.86086300 & 4.73236200 & -1.45137800 \\
\hline$H$ & -6.31163500 & 6.39287600 & -1.66694200 \\
\hline C & -7.16425100 & 5.50099100 & 0.09874700 \\
\hline
\end{tabular}

\begin{tabular}{|c|c|c|c|}
\hline $\mathrm{H}$ & -6.83333600 & 4.91233900 & 0.96348300 \\
\hline $\mathrm{H}$ & -7.43958900 & 6.48001700 & 0.50746800 \\
\hline C & -8.38628300 & 4.83521200 & -0.54037800 \\
\hline $\mathrm{H}$ & -8.15164800 & 3.81001900 & -0.84622900 \\
\hline $\mathrm{H}$ & -9.22763600 & 4.79187100 & 0.16175600 \\
\hline $\mathrm{H}$ & -8.71980900 & 5.39154700 & -1.42545100 \\
\hline C & -5.67624700 & 8.46724400 & -0.08115400 \\
\hline $\mathrm{H}$ & -6.53034200 & 8.07473400 & -0.63006700 \\
\hline $\mathrm{H}$ & -6.03861400 & 8.75418500 & 0.91542200 \\
\hline $\mathrm{H}$ & -5.37254700 & 9.39229100 & -0.58236400 \\
\hline C & -3.17558000 & 9.46861300 & 0.76866500 \\
\hline $\mathrm{H}$ & -2.22039600 & 9.72973700 & 1.22445300 \\
\hline $\mathrm{H}$ & -3.31308200 & 10.12772000 & -0.09850300 \\
\hline $\mathrm{H}$ & -3.95980000 & 9.73195900 & 1.48741300 \\
\hline C & -6.46277500 & -7.66787400 & 0.42528200 \\
\hline $\mathrm{H}$ & -7.33007600 & -7.06553700 & 0.15371900 \\
\hline $\mathrm{H}$ & -6.47068800 & -8.56554000 & -0.20676600 \\
\hline $\mathrm{H}$ & -6.61993800 & -8.01180100 & 1.45472300 \\
\hline C & -4.06934100 & -8.95321900 & 1.26533700 \\
\hline $\mathrm{H}$ & -4.75975600 & -8.99018800 & 2.11692900 \\
\hline $\mathrm{H}$ & -4.46096900 & -9.65530000 & 0.51780200 \\
\hline $\mathrm{H}$ & -3.11229800 & -9.34179500 & 1.61327100 \\
\hline $\mathrm{C}$ & -7.71213300 & 1.71168100 & 1.63144400 \\
\hline $\mathrm{H}$ & -8.01931000 & 0.70668900 & 1.93680100 \\
\hline $\mathrm{H}$ & -6.92730300 & 2.07948300 & 2.30481800 \\
\hline $\mathrm{H}$ & -8.56613200 & 2.39227200 & 1.66236400 \\
\hline C & -8.30578300 & -1.10046500 & -0.65566300 \\
\hline $\mathrm{H}$ & -8.61808100 & -0.08584000 & -0.92654500 \\
\hline $\mathrm{H}$ & -7.90732900 & -1.61383000 & -1.54089500 \\
\hline $\mathrm{H}$ & -9.15946200 & -1.66008600 & -0.26570900 \\
\hline C & -6.27562900 & -5.11926800 & -1.08682700 \\
\hline $\mathrm{H}$ & -7.22238500 & -5.09011200 & -0.52911000 \\
\hline $\mathrm{H}$ & -6.09991700 & -4.09881200 & -1.42437500 \\
\hline C & -6.45186400 & 256500 & -2.360 \\
\hline $\mathrm{H}$ & -5.50762400 & 393000 & -2.92053500 \\
\hline $\mathrm{H}$ & -6.63514200 & -7.02240300 & -2.10763500 \\
\hline C & -7.59179200 & 29500 & -3.23997400 \\
\hline $\mathrm{H}$ & -8.54941400 & -5.48271500 & -2.70484400 \\
\hline $\mathrm{H}$ & -7.41569400 & -4.40777200 & -3.53711400 \\
\hline $\mathrm{H}$ & -7.69857100 & -6.04423300 & -4.15411800 \\
\hline C & -1.46947500 & -7.75090500 & 0.53586600 \\
\hline $\mathrm{H}$ & -0.57902200 & -7.13577500 & 0.43410600 \\
\hline $\mathrm{H}$ & -1.32666200 & -8.32529800 & 1.46135100 \\
\hline C & -1.47185100 & -8.70768800 & -0.67570000 \\
\hline $\mathrm{H}$ & -2.34482200 & -9.36905700 & -0.65270200 \\
\hline $\mathrm{H}$ & -1.57057200 & -8.10448900 & -1.58775100 \\
\hline C & -0.19251500 & -9.54743000 & -0.74103800 \\
\hline $\mathrm{H}$ & 0.69724500 & -8.90880700 & -0.80183600 \\
\hline $\mathrm{H}$ & -0.08720700 & -10.17560300 & 0.15263600 \\
\hline $\mathrm{H}$ & -0.19196800 & -10.20767300 & -1.61592700 \\
\hline C & 1.84261900 & -5.02915500 & 4.07838800 \\
\hline $\mathrm{H}$ & 1.68519000 & -5.87801800 & 4.74819200 \\
\hline $\mathrm{H}$ & 2.78350800 & -4.52732200 & 4.32559000 \\
\hline $\mathrm{H}$ & 1.00865900 & -4.32303400 & 4.18143100 \\
\hline C & 4.97019300 & -5.40848300 & 2.18495000 \\
\hline $\mathrm{H}$ & 5.35386300 & -5.45812700 & 1.15702400 \\
\hline $\mathrm{H}$ & 5.80873500 & -5.42387800 & 2.88546500 \\
\hline $\mathrm{H}$ & 4.31002700 & -6.26227400 & 2.37324100 \\
\hline C & 7.34179700 & 1.07774000 & -1.55853500 \\
\hline C & 6.11226300 & 0.52377200 & -1.33283400 \\
\hline C & 6.10327000 & -0.92282100 & -1.16784000 \\
\hline C & 7.32913800 & -1.52666800 & -1.26046400 \\
\hline $\mathrm{Se}$ & 8.67264600 & -0.25146900 & -1.59455600 \\
\hline C & 7.69206200 & 2.53432700 & -1.63956300 \\
\hline $\mathrm{H}$ & 6.78025700 & 3.09890300 & -1.86998900 \\
\hline $\mathrm{H}$ & 8.39472500 & 2.71778900 & -2.46421800 \\
\hline C & 8.30051400 & 3.07164100 & -0.32840700 \\
\hline
\end{tabular}




$\begin{array}{lrrr}\mathrm{H} & 9.21158600 & 2.50360300 & -0.09850300 \\ \mathrm{H} & 7.59849000 & 2.86800300 & 0.48977300 \\ \mathrm{C} & 8.60638000 & 4.56941000 & -0.39232500 \\ \mathrm{H} & 9.04459700 & 4.92771500 & 0.54608900 \\ \mathrm{H} & 9.31283000 & 4.79679800 & -1.20026500 \\ \mathrm{H} & 7.69344200 & 5.14919800 & -0.57888100 \\ \mathrm{C} & 7.66963200 & -2.97973100 & -1.11053600 \\ \mathrm{H} & 8.30615400 & -3.31080700 & -1.94331300 \\ \mathrm{H} & 6.74311000 & -3.56324400 & -1.16971100 \\ \mathrm{C} & 8.38172600 & -3.29857400 & 0.21943500 \\ \mathrm{H} & 9.30761700 & -2.71143300 & 0.28066300 \\ \mathrm{H} & 7.74962700 & -2.95609700 & 1.04878200 \\ \mathrm{C} & 8.68708500 & -4.78985200 & 0.37532400 \\ \mathrm{H} & 9.19209000 & -4.99775300 & 1.32535200 \\ \mathrm{H} & 7.76462300 & -5.38356000 & 0.34938100 \\ \mathrm{H} & 9.33406900 & -5.14905800 & -0.43455600\end{array}$

\section{1-Se TS}

\begin{tabular}{|c|c|c|c|}
\hline C & 3.39045900 & 1.38759800 & -0.28349000 \\
\hline C & 4.47329800 & 2.29397200 & -0.09864800 \\
\hline C & 3.65320500 & -0.02657900 & -0.51728800 \\
\hline 0 & 2.64230700 & -0.99525900 & -0.62354500 \\
\hline C & 2.91290100 & -2.36267700 & -0.71639800 \\
\hline$C$ & 4.26027100 & -2.81405500 & -0.71489300 \\
\hline C & 5.26878400 & -1.85909900 & -0.59265900 \\
\hline C & 1.84802000 & -3.35283400 & -0.55208000 \\
\hline$\Omega$ & 2.19846700 & -4.58896400 & 0.10000200 \\
\hline C & 3.57353200 & -5.13179000 & -0.11576600 \\
\hline C & 4.52682700 & -4.26647200 & -0.74601900 \\
\hline C & 0.51350600 & -3.05165100 & -0.81355900 \\
\hline C & -0.54330700 & -3.79282700 & -0.25663200 \\
\hline C & 1.15682900 & -5.18365300 & 0.82105700 \\
\hline C & -1.94428100 & -3.51555700 & -0.50238000 \\
\hline C & 5.00192100 & -0.48274700 & -0.51618300 \\
\hline C & -0.18636900 & -4.77917500 & 0.70202700 \\
\hline C & -2.91456300 & -3.99081600 & 0.42720500 \\
\hline U & -4.24707600 & -3.52043100 & 0.34660800 \\
\hline & -2.40375000 & -2.78560600 & -1.62185300 \\
\hline & 5.82580200 & 1.80116800 & -0.18827600 \\
\hline & -2.50999800 & -4.91740300 & 1.45371400 \\
\hline & -3.72800200 & -2.41228600 & -1.73030000 \\
\hline & 6.07736900 & 0.46812300 & -0.37159700 \\
\hline & -1.20925100 & -5.34833800 & 1.54022900 \\
\hline & -4.64127900 & -2.66940100 & -0.67488200 \\
\hline & 1.60820900 & -0.68741500 & -0.52856400 \\
\hline & 6.29905900 & -2.16374700 & -0.50162000 \\
\hline & 0.28544100 & -2.16561600 & -1.39744000 \\
\hline 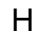 & 1.33607900 & -5.95674900 & 1.54306100 \\
\hline $\mathrm{H}$ & -4.94663600 & -3.79835700 & 1.12715300 \\
\hline$\dashv$ & -1.70287100 & -2.51709000 & -2.40667700 \\
\hline-1 & -4.06070700 & -1.81922400 & -2.57690500 \\
\hline & 5.67083100 & -4.79698200 & -1.39764100 \\
\hline C & 5.94924600 & -6.16817700 & -1.27819300 \\
\hline C & 5.14262000 & -6.98619800 & -0.46053200 \\
\hline C & 3.93446600 & -6.50149500 & 0.08006000 \\
\hline C & 2.10329000 & 1.92212000 & -0.20226400 \\
\hline & 1.84718500 & 3.24707400 & 0.15009500 \\
\hline & 2.92508500 & 4.15605700 & 0.36203000 \\
\hline & 4.21606900 & 3.64955700 & 0.16873600 \\
\hline & 0.47840300 & 3.72094400 & 0.30395100 \\
\hline & 0.20869100 & 5.12186700 & 0.30747700 \\
\hline & 1.33881100 & 6.07362400 & 0.26069200 \\
\hline & 2.63377600 & 5.58627300 & 0.62283500 \\
\hline & -0.56344500 & 2.81579600 & 0.45315600 \\
\hline & -1.90102700 & 3.20387500 & 0.52998000 \\
\hline & -1.12184900 & 5.51597800 & 0.50317300 \\
\hline & -2.97904300 & 2.24402900 & 0.4983820 \\
\hline
\end{tabular}

$\begin{array}{llll}-2.18366100 & 4.59618800 & 0.56656800\end{array}$ $\begin{array}{lll}-4.32645400 & 2.68348400 & 0.46970400\end{array}$ $\begin{array}{lll}-2.76433600 & 0.85738800 & 0.43566600\end{array}$ $\begin{array}{llll}-5.13737900 & 0.39596900 & -0.00249800\end{array}$ $\begin{array}{lll}-5.37082800 & 1.75770100 & 0.24642100\end{array}$ $\begin{array}{lll}-3.79022600 & -0.01903400 & 0.18555000\end{array}$ $\begin{array}{lll}-4.58688400 & 4.10098500 & 0.59166300\end{array}$ $\begin{array}{lll}-3.56226300 & 5.01392400 & 0.63847300\end{array}$ $\begin{array}{lll}1.26486100 & 1.27938100 & -0.43276800\end{array}$ $\begin{array}{llll}5.07468700 & 4.29668400 & 0.20619600\end{array}$ $\begin{array}{lll}-0.32730200 & 1.76336800 & 0.50737900\end{array}$ $\begin{array}{lll}-1.37559200 & 6.55771500 & 0.61816500\end{array}$ $\begin{array}{lll}-6.36192500 & 2.17299800 & 0.23548300\end{array}$ $\begin{array}{lll}3.57997600 & 6.50528200 & 1.16310800\end{array}$ $\begin{array}{llll}3.31457600 & 7.88350300 & 1.08846100\end{array}$ $\begin{array}{llll}1.19263000 & 7.45589600 & -0.05059500\end{array}$ $\begin{array}{llll}2.19959000 & 8.34945700 & 0.34916400\end{array}$ $\begin{array}{lll}-3.53172000 & -1.05793800 & 0.16814100\end{array}$ $\begin{array}{lll}-1.76755800 & 0.44507100 & 0.54584500\end{array}$ $\begin{array}{lll}-5.90471300 & 4.51528400 & 0.58392000\end{array}$ $\begin{array}{lll}-3.82082500 & 6.36680900 & 0.73629100\end{array}$ $\begin{array}{lll}-6.36922600 & 5.02693500 & 1.84062300\end{array}$ $\begin{array}{lll}-5.81645500 & 5.92837000 & 2.12313700\end{array}$ $\begin{array}{lll}-7.42626600 & 5.26413600 & 1.70009300\end{array}$ $\begin{array}{lll}-6.26816300 & 4.26795800 & 2.62692200\end{array}$ $\begin{array}{lll}-4.28743900 & 6.95853400 & -0.48290700\end{array}$ $\begin{array}{llll}-5.23524200 & 6.50894800 & -0.79803900\end{array}$ $\begin{array}{llll}-4.43279700 & 8.02065600 & -0.27060700\end{array}$ $\begin{array}{llll}-3.54005500 & 6.84464500 & -1.27956900\end{array}$ $\begin{array}{lll}4.82002700 & 6.02668500 & 1.90835900\end{array}$ $\begin{array}{lll}4.73003800 & 4.96320900 & 2.13799600\end{array}$ $\begin{array}{lll}4.85387200 & 6.51994800 & 2.88865100\end{array}$ $\begin{array}{llll}0.04056700 & 7.97898400 & -0.91276200\end{array}$ $\begin{array}{llll}-0.89200000 & 8.06986300 & -0.34574400\end{array}$ $\begin{array}{llll}0.27212100 & 9.00034500 & -1.21848000\end{array}$ $\begin{array}{llll}6.84869700 & 2.70823000 & -0.00431300\end{array}$ $\begin{array}{llll}7.36799900 & -0.01524900 & -0.44795600\end{array}$ $\begin{array}{llll}7.64885000 & 2.96339800 & -1.16577300\end{array}$ $\begin{array}{llll}8.17961600 & 2.06031400 & -1.48398700\end{array}$ $\begin{array}{lll}7.02586300 & 3.33387200 & -1.98969500\end{array}$ $\begin{array}{llll}8.36379600 & 3.73598500 & -0.87359900\end{array}$ $\begin{array}{lll}8.09154600 & 0.00592400 & 0.78966600\end{array}$ $\begin{array}{lll}7.56767300 & -0.58463700 & 1.55198800\end{array}$ $\begin{array}{lll}9.06232000 & -0.44888100 & 0.57907900\end{array}$ $\begin{array}{lll}8.22698100 & 1.03143400 & 1.14817300\end{array}$ $6.55871800-3.93865900-2.28976200$ $\begin{array}{lll}6.05719500 & -2.99880200 & -2.52590000\end{array}$ $\begin{array}{llll}6.68455100 & -4.44025200 & -3.25799600\end{array}$ $\begin{array}{lll}3.03993400 & -7.48044300 & 0.82149200\end{array}$ $\begin{array}{llll}2.01090000 & -7.36086600 & 0.47376600\end{array}$ $\begin{array}{lll}3.29891000 & -8.50756400 & 0.55766000\end{array}$ $\begin{array}{lll}-0.81586800 & -6.27714600 & 2.48009700\end{array}$ $\begin{array}{lll}-3.49112000 & -5.39597100 & 2.29495600\end{array}$ $\begin{array}{lll}-1.34707300 & -7.59321700 & 2.27590400\end{array}$ $\begin{array}{lll}-0.90976600 & -8.21985800 & 3.05676800\end{array}$ $\begin{array}{lll}-2.43856500 & -7.59670100 & 2.36013600\end{array}$ $\begin{array}{lll}-1.05389900 & -7.97825400 & 1.29077200\end{array}$ $\begin{array}{lll}-3.33651100 & -5.01862000 & 3.67058700\end{array}$ $\begin{array}{lll}-4.20797500 & -5.41996500 & 4.19309700\end{array}$ $\begin{array}{llll}-2.41900200 & -5.43775800 & 4.09548000\end{array}$ $\begin{array}{lll}-3.32100000 & -3.92594600 & 3.77045600\end{array}$ $\begin{array}{llll}7.10404800 & -6.81135800 & -2.02388500\end{array}$ $\begin{array}{lll}7.46002800 & -6.20133900 & -2.85374500\end{array}$ $\begin{array}{llll}7.96509800 & -6.99273500 & -1.36632600\end{array}$ $\begin{array}{lll}6.81374900 & -7.78052200 & -2.44181300\end{array}$ $\begin{array}{llll}5.58528100 & -8.42665400 & -0.25798700\end{array}$ $\begin{array}{lll}5.24126500 & -9.07658200 & -1.07546900\end{array}$ 


\section{Helicene 3 GS}

$\begin{array}{llll}\text { C } & -0.72465200 & -3.32032900 & -0.06230700\end{array}$

$\begin{array}{llll}\text { C } & -1.49696000 & -2.15359300 & -0.07484700\end{array}$

$6.67510600-8.50425000 \quad-0.22772100$

$\begin{array}{lll}5.22088800 & -8.85649300 & 0.67208700\end{array}$

$\begin{array}{llll}3.09407200 & -7.34013000 & 2.36352400\end{array}$

$2.94875600 \quad-6.28940800 \quad 2.63922400$

$\begin{array}{lll}2.25020800 & -7.89400800 & 2.79777800\end{array}$

$\begin{array}{lll}4.40255600 & -7.82403400 & 2.99694000\end{array}$

$\begin{array}{lll}4.55400200 & -8.89887700 & 2.83897900\end{array}$

$\begin{array}{lll}5.26417600 & -7.29464300 & 2.57456900\end{array}$

$\begin{array}{lll}4.39865200 & -7.64720500 & 4.07843700\end{array}$

$7.96155200-3.63399000-1.71836500$

$\begin{array}{lll}7.87789500 & -3.33905400 & -0.66458000\end{array}$

$8.56553200-4.54884200-1.71610100$

$8.67888500-2.53128200-2.50206300$

$8.73260000-2.77687700 \quad-3.57026600$

$8.15243400-1.57673400-2.39379100$

$9.70432200-2.38939100-2.14057600$

$\begin{array}{lll}4.22284000 & 8.89679800 & 1.76304600\end{array}$

$\begin{array}{lll}4.85051400 & 8.44548800 & 2.53125100\end{array}$

$\begin{array}{lll}4.89493900 & 9.39209200 & 1.04980100\end{array}$

$\begin{array}{lll}3.63860300 & 9.68162000 & 2.25398900\end{array}$

$\begin{array}{lll}2.08477100 & 9.82685500 & 0.01136600\end{array}$

$\begin{array}{lll}1.16056800 & 10.27117700 & 0.40030000\end{array}$

$\begin{array}{lll}2.91512000 & 10.41159900 & 0.40489600\end{array}$

$2.08265400 \quad 9.98055000 \quad-1.07456600$

$\begin{array}{lll}6.16464600 & 6.28648000 & 1.19384700\end{array}$

$\begin{array}{lll}6.09578300 & 5.97215900 & 0.14470100\end{array}$

$\begin{array}{lll}6.36063900 & 7.36435700 & 1.16176600\end{array}$

$\begin{array}{lll}7.32987700 & 5.56026700 & 1.87209900\end{array}$

$\begin{array}{lll}7.40177300 & 5.83375900 & 2.93241200\end{array}$

$\begin{array}{lll}7.19950900 & 4.47463800 & 1.80510100\end{array}$

$\begin{array}{llll}8.28579000 & 5.81313500 & 1.39777000\end{array}$

$\begin{array}{lll}-0.21597600 & 7.18349000 & -2.21119900\end{array}$

$\begin{array}{llll}-0.55520600 & 6.16940700 & -1.98219400\end{array}$

$\begin{array}{llll}-1.04222300 & 7.67397500 & -2.74455800\end{array}$

$\begin{array}{lll}1.01561600 & 7.11812000 & -3.12050600\end{array}$

$\begin{array}{lll}1.37162800 & 8.12397500 & -3.37894900\end{array}$

$\begin{array}{lll}1.83855400 & 6.59057700 & -2.62655100\end{array}$

$\begin{array}{llll}0.79001400 & 6.59165500 & -4.05472800\end{array}$

$-7.07088900-2.71272500-1.09555000$

$\begin{array}{lll}-5.97134900 & -1.99252500 & -0.72011700\end{array}$

$-6.15904600 \quad-0.52872500 \quad-0.59241200$

$\begin{array}{lll}-7.42685800 & -0.13014000 & -0.98735800\end{array}$

$\begin{array}{lll}-8.52639000 & -1.58688700 & -1.41678000\end{array}$

$-7.20069000-4.19720800-1.27430600$

$-6.20075700 \quad-4.63618100 \quad-1.36688700$

$\begin{array}{lll}-7.73390300 & -4.42325100 & -2.20820600\end{array}$

$\begin{array}{lll}-7.93885600 & -4.87028300 & -0.09989200\end{array}$

$\begin{array}{lll}-8.93056400 & -4.41119900 & 0.01042000\end{array}$

$\begin{array}{lll}-7.39451200 & -4.64914700 & 0.82737400\end{array}$

$\begin{array}{lll}-8.07092800 & -6.38365200 & -0.28285100\end{array}$

$\begin{array}{lll}-8.59938000 & -6.84299200 & 0.56006100\end{array}$

$\begin{array}{lll}-8.62591900 & -6.62456700 & -1.19814900\end{array}$

$-7.08426300 \quad-6.85686800-0.35809100$

$\begin{array}{lll}-8.08321900 & 1.21896000 & -1.10595100\end{array}$

$\begin{array}{lll}-8.80255800 & 1.19551500 & -1.93571200\end{array}$

$\begin{array}{lll}-7.34068200 & 1.97277900 & -1.38596700\end{array}$

$\begin{array}{lll}-8.83624300 & 1.65635200 & 0.16907100\end{array}$

$\begin{array}{lll}-9.68406100 & 0.97597000 & 0.32095000\end{array}$

$\begin{array}{lll}-8.18525100 & 1.52412600 & 1.04186600\end{array}$

$\begin{array}{lll}-9.30667100 & 3.11054700 & 0.08629400\end{array}$

$\begin{array}{lll}-9.85471300 & 3.40596900 & 0.98837000\end{array}$

$\begin{array}{llll}-8.44755000 & 3.78282300 & -0.02761400\end{array}$

$\begin{array}{lll}-9.96974800 & 3.26498300 & -0.77415000\end{array}$
$-2.88510700-2.15843900-0.21054500$

$\begin{array}{lll}-3.58581200 & -3.40023600 & -0.30740200\end{array}$

$-2.82038800 \quad-4.56721700 \quad-0.22508800$

$-3.64806700 \quad-0.91301600-0.22487500$

$\begin{array}{lll}-5.05216300 & -0.96740700 & 0.02901500\end{array}$

$\begin{array}{lll}-5.73215300 & -2.26760800 & 0.19845700\end{array}$

$\begin{array}{lll}-5.06576400 & -3.42366600 & -0.30474200\end{array}$

$\begin{array}{llll}-3.04196700 & 0.31069000 & -0.50784000\end{array}$

$\begin{array}{lll}-3.74168200 & 1.52535200 & -0.53443000\end{array}$

$\begin{array}{llll}-5.76414600 & 0.22836600 & -0.07108300\end{array}$

$\begin{array}{lll}-3.10924700 & 2.80974500 & -0.78621900\end{array}$

$-1.42105400 \quad-4.55939700 \quad-0.13231400$

$\begin{array}{llll}0.72471100 & -3.32031600 & 0.06237100\end{array}$

$\begin{array}{lll}1.49699900 & -2.15356700 & 0.07490400\end{array}$

$\begin{array}{llll}2.88514800 & -2.15838900 & 0.21058700\end{array}$

$\begin{array}{llll}3.58587700 & -3.40017400 & 0.30743600\end{array}$

$\begin{array}{llll}2.82047000 & -4.56716700 & 0.22514000\end{array}$

$\begin{array}{lll}3.64808400 & -0.91295200 & 0.22490700\end{array}$

$\begin{array}{llll}5.05217800 & -0.96731700 & -0.02900500\end{array}$

$\begin{array}{llll}5.73218700 & -2.26750600 & -0.19846500\end{array}$

$\begin{array}{lll}5.06583000 & -3.42357800 & 0.30474300\end{array}$

$\begin{array}{llll}3.04196500 & 0.31074300 & 0.50788100\end{array}$

$\begin{array}{lll}3.74165900 & 1.52541800 & 0.53445800\end{array}$

$\begin{array}{llll}5.76414000 & 0.22846900 & 0.07108500\end{array}$

$\begin{array}{lll}3.10920300 & 2.80980000 & 0.78624900\end{array}$

$\begin{array}{lll}1.42113400 & -4.55937200 & 0.13237800\end{array}$

$\begin{array}{lll}-5.14919900 & 1.46464700 & -0.31972300\end{array}$

$\begin{array}{lll}5.14917400 & 1.46473800 & 0.31973100\end{array}$

$\begin{array}{llll}3.91537700 & 3.98537100 & 0.83608500\end{array}$

$\begin{array}{llll}-3.91544300 & 3.98530100 & -0.83607500\end{array}$

$\begin{array}{llll}3.32251600 & 5.24226400 & 1.07008700\end{array}$

$\begin{array}{lll}1.71782300 & 2.96123400 & 0.98639400\end{array}$

$\begin{array}{lll}-1.71786700 & 2.96120500 & -0.98634500\end{array}$

$\begin{array}{llll}-1.94803800 & 5.36512500 & -1.26266600\end{array}$

$\begin{array}{lll}-3.32260300 & 5.24220300 & -1.07007700\end{array}$

$\begin{array}{lll}-0.68083900 & -5.79552800 & -0.06384700\end{array}$

$\begin{array}{llll}5.34168500 & 3.87975400 & 0.64041900\end{array}$

$\begin{array}{lll}1.14794000 & 4.19425400 & 1.22480800\end{array}$

$-1.14800500 \quad 4.19423400-1.22476000$

$\begin{array}{lll}0.68094100 & -5.79551600 & 0.06392000\end{array}$

$\begin{array}{lll}5.93168500 & 2.67190400 & 0.38370200\end{array}$

$\begin{array}{lll}1.94795100 & 5.36516000 & 1.26269300\end{array}$

$\begin{array}{llll}-5.34175200 & 3.87965800 & -0.64042800\end{array}$

$\begin{array}{lll}-5.93173200 & 2.67179800 & -0.38371200\end{array}$

$\begin{array}{lll}-1.00054300 & -1.20294300 & 0.07223700\end{array}$

$\begin{array}{lll}-3.29408000 & -5.53207800 & -0.18653600\end{array}$

$\begin{array}{lll}-1.98934900 & 0.31386100 & -0.75948700\end{array}$

$\begin{array}{lll}-6.83838300 & 0.22711400 & -0.01282200\end{array}$

$\begin{array}{lll}1.00056400 & -1.20292600 & -0.07217600\end{array}$

$\begin{array}{llll}3.29417500 & -5.53202100 & 0.18659100\end{array}$

$\begin{array}{lll}1.98935100 & 0.31389300 & 0.75954400\end{array}$

$\begin{array}{llll}6.83837700 & 0.22723500 & 0.01280600\end{array}$

$\begin{array}{lll}3.95428600 & 6.12233200 & 1.08653600\end{array}$

$\begin{array}{lll}1.06657000 & 2.09437200 & 0.94670700\end{array}$

$\begin{array}{lll}-1.06659700 & 2.09435600 & -0.94664500\end{array}$

$\begin{array}{lll}-3.95439000 & 6.12225900 & -1.08654200\end{array}$

$\begin{array}{llll}0.07622500 & 4.28245700 & 1.36430400\end{array}$

$\begin{array}{lll}-0.07629000 & 4.28245800 & -1.36424300\end{array}$

$\begin{array}{llll}1.32862900 & 6.63263700 & 1.47792200\end{array}$

$\begin{array}{lll}-1.32873900 & 6.63261300 & -1.47789700\end{array}$

$\begin{array}{lll}0.72993600 & 7.67248100 & 1.65304100\end{array}$

$\begin{array}{lll}-0.73006700 & 7.67246800 & -1.65301800\end{array}$

$\begin{array}{lll}0.00864600 & 8.92901500 & 1.83779600\end{array}$

$\begin{array}{lll}-0.52492100 & 8.91164100 & 2.79954000\end{array}$

$\begin{array}{lll}-0.76869000 & 9.00537300 & 1.06414500\end{array}$

$\begin{array}{llll}-0.00880400 & 8.92901700 & -1.83777400\end{array}$

$\begin{array}{llll}0.76852300 & 9.00539800 & -1.06411600\end{array}$ 


\begin{tabular}{|c|c|c|c|}
\hline $\mathrm{H}$ & 0.52477300 & 8.91164900 & -2.79951300 \\
\hline C & 5.84737800 & -4.54915700 & 0.69446700 \\
\hline C & 7.19995800 & -4.59488500 & 0.32059600 \\
\hline C & 7.73615500 & -3.60389100 & -0.53570400 \\
\hline C & 7.03343200 & -2.40931400 & -0.75984800 \\
\hline C & -5.84728100 & -4.54925600 & -0.69449200 \\
\hline C & -7.19987000 & -4.59501100 & -0.32065400 \\
\hline C & -7.03340800 & -2.40944400 & 0.75981000 \\
\hline C & -7.73610400 & -3.60403300 & 0.53564200 \\
\hline O & 6.07885500 & 5.04404600 & 0.68435500 \\
\hline $\mathrm{O}$ & 7.29476200 & 2.56144800 & 0.19674800 \\
\hline C & 6.96118800 & 5.14589500 & 1.81057300 \\
\hline & 6.39629400 & 5.08609700 & 2.74957400 \\
\hline $\mathrm{H}$ & 7.72049600 & 4.35765600 & 1.78790700 \\
\hline $\mathrm{H}$ & 7.43760600 & 6.12612900 & 1.73390700 \\
\hline C & 7.77229800 & 3.14623200 & -1.02229600 \\
\hline $\mathrm{H}$ & 7.61192500 & 4.22902300 & -1.02832400 \\
\hline $\mathrm{H}$ & 8.84027800 & 2.92300800 & -1.06831600 \\
\hline $\mathrm{H}$ & 7.26981900 & 2.69527600 & -1.88744300 \\
\hline C & 7.61614200 & -1.37445400 & -1.71860000 \\
\hline $\mathrm{H}$ & 6.84439300 & -0.65604800 & -2.00753300 \\
\hline $\mathrm{H}$ & 7.87198500 & -1.90314100 & -2.64762700 \\
\hline C & 5.28073800 & -5.65328800 & 1.57860500 \\
\hline $\mathrm{H}$ & 4.32609800 & -5.33506700 & 2.00336600 \\
\hline $\mathrm{H}$ & 5.94436400 & -5.78126400 & 2.44449000 \\
\hline $\mathrm{O}$ & 1.38996700 & -6.97440000 & 0.17114200 \\
\hline $\mathrm{O}$ & -1.38984300 & -6.97442600 & -0.17106400 \\
\hline C & 1.47645500 & -7.73648600 & -1.03976100 \\
\hline $\mathrm{H}$ & 0.48525500 & -8.06761800 & -1.36627200 \\
\hline $\mathrm{H}$ & 1.94870900 & -7.14534500 & -1.83475700 \\
\hline $\mathrm{H}$ & 2.10332600 & -8.60047300 & -0.80721200 \\
\hline C & -1.47632200 & -7.73650400 & 1.03984400 \\
\hline $\mathrm{H}$ & -1.94859000 & -7.14536600 & 1.83483400 \\
\hline $\mathrm{H}$ & -2.10317600 & -8.60050500 & 0.80730000 \\
\hline $\mathrm{H}$ & -0.48511700 & -8.06761500 & 1.36636100 \\
\hline C & -5.28059900 & -5.65336900 & -1.57862500 \\
\hline 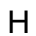 & -4.32595600 & -5.33512400 & -2.00336300 \\
\hline & -5.94420400 & -5.78135300 & -2.44452700 \\
\hline 0 & -7.61615800 & -1.37460200 & 1.71855600 \\
\hline $\mathrm{H}$ & -6.84442800 & -0.65618500 & 2.00751300 \\
\hline $\mathrm{H}$ & -7.87201500 & -1.90330000 & 2.64757300 \\
\hline $\mathrm{O}$ & -7.29481000 & 2.56131800 & -0.19677700 \\
\hline O & -6.07894300 & 5.04393500 & -0.68438000 \\
\hline C & -7.77237400 & 3.14610900 & 1.02225200 \\
\hline $\mathrm{H}$ & -7.26990200 & 2.69517200 & 1.88741300 \\
\hline $\mathrm{H}$ & -7.61201900 & 4.22890300 & 1.02827000 \\
\hline $\mathrm{H}$ & -8.84035200 & 2.92287000 & 1.06825900 \\
\hline C & -6.96127000 & 5.14575800 & -1.81060500 \\
\hline $\mathrm{H}$ & -7.72056100 & 4.35750300 & -1.78794000 \\
\hline $\mathrm{H}$ & -7.43770800 & 6.12598300 & -1.73394800 \\
\hline 1 & -6.39636700 & 5.08596600 & -2.74960100 \\
\hline & -8.87267000 & -0.60491800 & 1.25821900 \\
\hline 1 & -8.62453800 & 0.07601700 & 0.43620700 \\
\hline$H$ & -9.61405700 & -1.30111400 & 0.85236900 \\
\hline C & -9.49607300 & 0.19569400 & 2.40601200 \\
\hline 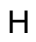 & -9.83458500 & -0.46641000 & 3.21292800 \\
\hline $\mathrm{H}$ & -8.77091700 & 0.89582100 & 2.83800900 \\
\hline $\mathrm{H}$ & -10.36091300 & 0.77715300 & 2.06601300 \\
\hline C & -5.10640800 & -7.03270000 & -0.90296200 \\
\hline & -4.64101800 & -6.91056300 & 0.08327000 \\
\hline 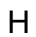 & -6.09034300 & -7.47299400 & -0.70709200 \\
\hline & -4.26458000 & -7.98956200 & -1.75198000 \\
\hline & -3.23791900 & -7.61991700 & -1.84755000 \\
\hline & -4.21970500 & -8.98734500 & -1.29913100 \\
\hline 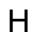 & -4.68714700 & -8.09951500 & -2.75868100 \\
\hline & -9.08975900 & -3.86838300 & 1.16777400 \\
\hline & -9.26144500 & -3.26598800 & 2.06099100 \\
\hline
\end{tabular}

$\begin{array}{lrrr}\mathrm{H} & -9.91621100 & -3.65916600 & 0.47484300 \\ \mathrm{H} & -9.18628300 & -4.91751200 & 1.46501500 \\ \mathrm{C} & -8.11428900 & -5.71761400 & -0.77201200 \\ \mathrm{H} & -7.75473700 & -6.20896100 & -1.67719000 \\ \mathrm{H} & -8.22246300 & -6.49546600 & -0.00382900 \\ \mathrm{H} & -9.12046300 & -5.34372100 & -0.98678100 \\ \mathrm{C} & 5.10656400 & -7.03261700 & 0.90293400 \\ \mathrm{H} & 4.64114400 & -6.91048400 & -0.08328500 \\ \mathrm{H} & 6.09050700 & -7.47288300 & 0.70703300 \\ \mathrm{C} & 4.26478400 & -7.98951000 & 1.75196500 \\ \mathrm{H} & 4.68738200 & -8.09946400 & 2.75865200 \\ \mathrm{H} & 3.23811700 & -7.61989100 & 1.84756900 \\ \mathrm{H} & 4.21992000 & -8.98728800 & 1.29910600 \\ \mathrm{C} & 8.87265200 & -0.60475200 & -1.25828800 \\ \mathrm{H} & 8.62453000 & 0.07616800 & -0.43626100 \\ \mathrm{H} & 9.61406300 & -1.30093800 & -0.85246600 \\ \mathrm{C} & 9.49600800 & 0.19588500 & -2.40608800 \\ \mathrm{H} & 10.36085000 & 0.77735200 & -2.06610800 \\ \mathrm{H} & 9.83450600 & -0.46620500 & -3.21302300 \\ \mathrm{H} & 8.77082900 & 0.89600700 & -2.83805500 \\ \mathrm{C} & 8.11440700 & -5.71747700 & 0.77191900 \\ \mathrm{H} & 7.75488500 & -6.20884400 & 1.67709900 \\ \mathrm{H} & 8.22257700 & -6.49531700 & 0.00372300 \\ \mathrm{H} & 9.12057900 & -5.34357000 & 0.98667100 \\ \mathrm{C} & 9.08980200 & -3.86820900 & -1.16786800 \\ \mathrm{H} & 9.26145500 & -3.26580400 & -2.06108400 \\ \mathrm{H} & 9.91626400 & -3.65898000 & -0.47495400 \\ \mathrm{H} & 9.18634100 & -4.91733400 & -1.46511900 \\ \mathrm{C} & -0.91513900 & 10.17644600 & -1.78625100 \\ \mathrm{H} & -1.67298300 & 10.09899100 & -2.57545200 \\ \mathrm{H} & -1.46184500 & 10.18395300 & -0.83500000 \\ \mathrm{C} & -0.11698500 & 11.47298500 & -1.94253200 \\ \mathrm{H} & 0.62501800 & 11.57885800 & -1.14184500 \\ \mathrm{H} & 0.42017100 & 11.49417700 & -2.89866600 \\ \mathrm{C} & 0.91495200 & 10.17646400 & 1.78625300 \\ \mathrm{H} & 1.46164600 & 10.18397700 & 0.83499600 \\ \mathrm{H} & 1.67280700 & 10.09903200 & 2.57544600 \\ \mathrm{C} & 0.11676800 & 11.47298600 & 1.94253500 \\ \mathrm{H} & -0.62524700 & 11.57883500 & 1.14185600 \\ \mathrm{H} & -0.42037600 & 11.49417100 & 2.89867500 \\ \mathrm{H} & -0.77282600 & 12.34990700 & -1.90749100 \\ \mathrm{H} & 0.77258800 & 12.34992300 & 1.90747900 \\ & & & \end{array}$

\begin{tabular}{lrrr}
\multicolumn{4}{l}{ Helicene 3 TS } \\
C & -3.44525200 & -0.54597300 & -1.32264600 \\
C & -2.30179300 & -1.35333000 & -1.37877300 \\
C & -2.32852800 & -2.71939200 & -1.10169500 \\
C & -3.57774800 & -3.36161200 & -0.86928300 \\
C & -4.72983100 & -2.57488700 & -0.87793900 \\
C & -1.09672000 & -3.36896700 & -0.64247000 \\
C & -1.27162800 & -4.35368300 & 0.38559300 \\
C & -2.46509700 & -5.23534000 & 0.31179000 \\
C & -3.57593400 & -4.78536200 & -0.46841200 \\
C & -4.69174300 & -1.18256000 & -1.06800900 \\
C & -3.36481400 & 0.90777800 & -1.27791400 \\
C & -2.15226200 & 1.59190500 & -1.37429400 \\
C & -2.01097900 & 2.93802600 & -1.04166200 \\
C & -3.14555000 & 3.69245000 & -0.64073600 \\
C & -4.37155600 & 3.02299300 & -0.58280500 \\
C & -0.67374500 & 3.46881500 & -0.79620500 \\
C & -0.53618300 & 4.51204600 & 0.17609600 \\
C & -1.67113700 & 5.46834900 & 0.32781000 \\
C & -2.94599100 & 5.10030600 & -0.22878000 \\
C & 0.44863800 & 2.81288900 & -1.28427000 \\
C & 1.71348600 & 2.95012400 & -0.70356700 \\
C & 0.68732000 & 4.53167900 & 0.86016000 \\
C & -4.51176100 & 1.66117700 & -0.90298200
\end{tabular}




\begin{tabular}{|c|c|c|c|}
\hline ; & 1.79559000 & 3.74942200 & 0.47157500 \\
\hline C & -5.87338000 & -0.37382100 & -0.87717900 \\
\hline C & -5.78031500 & 0.98930200 & -0.76637100 \\
\hline $\mathrm{H}$ & -1.33392200 & -0.87775000 & -1.45565500 \\
\hline $\mathrm{H}$ & -5.68262400 & -3.00502600 & -0.61207900 \\
\hline $\mathrm{H}$ & -1.25768200 & 1.02450700 & -1.58398900 \\
\hline $\mathrm{H}$ & -5.25033600 & 3.52210700 & -0.20981400 \\
\hline $\mathrm{H}$ & 0.31667300 & 2.10667300 & -2.09391000 \\
\hline $\mathrm{H}$ & 0.82093300 & 5.12180700 & 1.74982400 \\
\hline C & -3.96089100 & 6.07767500 & -0.41326300 \\
\hline C & -1.51028300 & 6.78804100 & 0.84051200 \\
\hline C & -2.59008100 & 7.69186300 & 0.79590700 \\
\hline C & -3.77373600 & 7.37073200 & 0.10585700 \\
\hline C & -4.61053000 & -5.68025100 & -0.83711400 \\
\hline C & -2.47986200 & -6.55849000 & 0.83592800 \\
\hline C & -3.57803400 & -7.39659700 & 0.56243200 \\
\hline C & -4.59750400 & -6.98909800 & -0.32133000 \\
\hline C & 2.88098400 & 2.25442400 & -1.19976100 \\
\hline & 3.01791800 & 3.72713300 & 1.23855800 \\
\hline & 4.09658200 & 2.98903700 & 0.81627400 \\
\hline & 4.08194700 & 2.27787100 & -0.43738800 \\
\hline & 2.88776900 & 1.55914200 & -2.42838200 \\
\hline & 1.99896100 & 1.54402500 & -3.04987900 \\
\hline & 5.24595800 & 1.64356500 & -0.92296500 \\
\hline & 5.23081300 & 0.95927000 & -2.13408500 \\
\hline & 4.02433900 & 0.93887800 & -2.88675600 \\
\hline & 4.02196800 & 0.44095300 & -3.85118800 \\
\hline & 0.17375300 & -2.85275600 & -0.89752000 \\
\hline 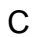 & 1.23695400 & -3.03076200 & 0.00419100 \\
\hline C & -0.28601300 & -4.37253300 & 1.37251900 \\
\hline$r$ & 0.92409500 & -3.66634400 & 1.24069400 \\
\hline $\mathrm{H}$ & 0.31508200 & -2.19014600 & -1.74613100 \\
\hline & -0.44098200 & -4.89081000 & 2.30374200 \\
\hline & 1.83685200 & -3.54854200 & 2.34581300 \\
\hline C & 2.98875000 & -2.81917000 & 2.22901400 \\
\hline C & 6.40055900 & 0.51791500 & -2.83024500 \\
\hline C & 7.27548400 & 0.16552000 & -3.59385200 \\
\hline 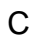 & 8.34710700 & -0.29332100 & -4.47589700 \\
\hline & 7.90434800 & -0.80495600 & -5.34332900 \\
\hline & 8.89040800 & 0.57169200 & -4.88472800 \\
\hline & 6.15796200 & 1.70512500 & -0.34372800 \\
\hline & 2.57094100 & -2.49096200 & -0.19197400 \\
\hline & 3.40247500 & -2.30010200 & 0.94796200 \\
\hline & 3.12907300 & -2.21864800 & -1.46023900 \\
\hline & 2.54493000 & -2.43852600 & -2.34941700 \\
\hline & 4.66932400 & -1.71558500 & 0.78968400 \\
\hline & 5.21084500 & -1.43798700 & -0.47376700 \\
\hline & 4.43190200 & -1.77313200 & -1.60754400 \\
\hline & 4.86518300 & -1.68071400 & -2.59581000 \\
\hline & 6.60093400 & -1.11101200 & -0.46673600 \\
\hline & 7.76683400 & -0.84287500 & -0.26976500 \\
\hline & 9.17921300 & -0.49945800 & -0.14126200 \\
\hline & 9.45116200 & 0.18739600 & -0.95612200 \\
\hline & 9.34473100 & 0.05802700 & 0.79210400 \\
\hline & 5.27912600 & -1.55729400 & 1.67163700 \\
\hline & 3.01815700 & 4.40936500 & 2.43311600 \\
\hline & 5.24361000 & 2.90903800 & 1.58197000 \\
\hline & 5.13500900 & 1.94511900 & 2.64003100 \\
\hline & 6.10608300 & 1.92830400 & 3.14091600 \\
\hline & 4.35375700 & 2.23782800 & 3.35152700 \\
\hline & 4.91127300 & 0.94953000 & 2.23627100 \\
\hline & 4.03490000 & 5.40817600 & 2.58933100 \\
\hline & 3.83205100 & 5.89177600 & 3.54719300 \\
\hline & 5.03387000 & 4.96548300 & 2.59356500 \\
\hline & 3.96988000 & 6.15167300 & 1.78425700 \\
\hline & -0.19636200 & 7.33420000 & 1.37573200 \\
\hline & & 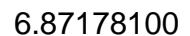 & \\
\hline
\end{tabular}

$\mathrm{H}$

$\mathrm{H}$

C

$\mathrm{H}$

$\mathrm{H}$

C

$\mathrm{H}$

$\mathrm{H}$

O

O

C

$\mathrm{H}$

$\mathrm{H}$

$\mathrm{H}$

C

$\mathrm{H}$

$\mathrm{H}$

$\mathrm{H}$

O

O

C

$\mathrm{H}$

$\mathrm{H}$

C

$\mathrm{H}$

$\mathrm{H}$

$\mathrm{H}$

C

$\mathrm{H}$

$\mathrm{H}$

$\mathrm{H}$

C

$\mathrm{H}$

$\mathrm{H}$

$\mathrm{H}$

C

$\mathrm{H}$

$\mathrm{H}$

C

H

$\mathrm{H}$

$\mathrm{H}$

C

$\mathrm{H}$

C

$\mathrm{H}$

$\mathrm{H}$

C

$\mathrm{H}$

$\mathrm{H}$

$\mathrm{H}$

C

$\mathrm{H}$

$\mathrm{H}$

C

$\mathrm{H}$

$\mathrm{H}$

$\mathrm{H}$

C

$\mathrm{H}$

$\mathrm{H}$

$\mathrm{H}$

C

$\mathrm{H}$
$-0.12521300$

$-5.21780900$

$-5.07939800$

$-5.36085700$

$-5.68595300$

$-5.38244500$

$-5.75580900$

$-1.32285000$

$-0.38206900$

$-1.22072600$

$-6.88970700$

$-7.07197700$

$-7.45754600$

$-8.27868600$

$-7.83514700$

$-6.71304400$

$-8.04739400$

$-8.89819700$

$-8.35865600$

$-7.64951900$

1.51099200

3.81304400

4.57248100

5.19638200

3.91220000

5.21315000

1.01680600

0.77250600

1.77951700

0.11213700

$-4.80829700$

$-5.52705300$

$-5.38324200$

$-4.33580000$

$-2.47989200$

$-2.18365700$

$-3.43907300$

$-1.75839100$

$-6.51630600$

$-6.33678400$

$-6.79418100$

$-7.67214600$

$-7.84356000$

$-7.45706500$

$-8.60591900$

$-0.01751900$

$-0.00706000$

$-7.09478200$

$-7.47137000$

$-7.04377300$

$-8.07583000$

$-8.16692000$

$-9.07638000$

$-7.73482000$

$-1.46152600$

$-2.29791200$

$-1.74598000$

$-0.17729700$

0.63660100

$-0.32334900$

0.14516700

$-5.65139400$

$-6.38657700$

$-6.20734800$

$-5.20264600$

$-3.66007900$

$-3.07422300$
$8.39805800 \quad 1.12540100$

$5.79646600-1.22764400$

$4.90276600-1.83808200$

$6.60872900-1.95173100$

$-5.28634800-1.84059100$

$-4.38418100-2.37624500$

$-6.06475100-2.61194300$

$-7.13822600 \quad 1.63322100$

$-6.69255200 \quad 1.30204500$

$-8.20623600 \quad 1.41320300$

$1.77334600-0.52245300$

$-1.03513000-0.70725400$

$\begin{array}{ll}1.62426700 & 0.78570600\end{array}$

$2.34274300 \quad 0.84068000$

$0.60806800 \quad 0.93822800$

$1.85903300 \quad 1.55690800$

$-0.77836600-1.72560500$

$-1.42464900-1.49887000$

$0.27103600-1.72033700$

$-1.03570900-2.71547600$

$-4.17946100 \quad 3.52998600$

$-2.60791400 \quad 3.31430300$

$-3.76001800 \quad 3.70631100$

$-3.44231000 \quad 4.54531300$

$-4.57524400 \quad 4.01995600$

$-4.098259002 .88167000$

$-3.29988800 \quad 4.54905200$

$-3.93336600 \quad 5.40507600$

$\begin{array}{ll}-2.56642400 & 4.83098300\end{array}$

$\begin{array}{ll}-2.78159400 & 4.20649800\end{array}$

$8.46594100-0.08432700$

$8.23836800-0.87035600$

$8.64203500 \quad 0.83497700$

$9.41709600-0.35007300$

$9.07621400 \quad 1.41426400$

$9.83262700 \quad 0.67315900$

$9.39799600 \quad 1.82896400$

$9.12397500 \quad 2.22905900$

$5.65287300-0.40218200$

$5.00482100 \quad 0.46524800$

$\begin{array}{ll}6.62614800 & 0.01851400\end{array}$

$5.08910100-1.23373100$

$5.69485000-2.13255300$

$4.06174600-1.54752500$

$5.07526800-0.65876200$

$7.18678900 \quad 2.90116000$

$6.12542700 \quad 3.17700100$

$-5.06657200-1.25056900$

$-6.00437300-0.82596800$

$-4.36377300-0.41065300$

$-4.53852700-2.30026700$

$-5.23440700-3.14374100$

$-4.39147700-1.87731500$

$-3.57610700-2.69972000$

$\begin{array}{ll}-6.96655000 & 3.16253400\end{array}$

$\begin{array}{ll}-7.57973100 & 3.52154100\end{array}$

$-5.93215900 \quad 3.39395600$

$\begin{array}{ll}-7.33312400 & 3.91263100\end{array}$

$\begin{array}{ll}-6.64615700 & 3.65342600\end{array}$

$-7.28326400 \quad 4.99815500$

$-8.352469003 .66564300$

$-8.00772500-0.71559800$

$\begin{array}{ll}-8.15983000 & 0.08640600\end{array}$

$-7.71820300-1.60705100$

$-8.98483200-0.92242900$

$-8.79448300 \quad 1.15134800$

$\begin{array}{ll}-8.90603700 & 2.06275700\end{array}$ 


$\begin{array}{cccc}\mathrm{H} & -4.69127600 & -9.05437900 & 1.40771200 \\ \mathrm{H} & -3.30743000 & -9.55667000 & 0.44167400 \\ \mathrm{H} & -0.89184500 & 7.60797300 & 3.41276900 \\ \mathrm{C} & 1.25717200 & 7.87100100 & 3.39997800 \\ \mathrm{H} & 1.39916400 & 7.72281900 & 4.47656800 \\ \mathrm{H} & 2.13927900 & 7.47408100 & 2.88616600 \\ \mathrm{H} & 1.22385400 & 8.95133900 & 3.21069600 \\ \mathrm{C} & 9.35256700 & -1.24339400 & -3.79128800 \\ \mathrm{H} & 9.84990500 & -0.70378100 & -2.97755500 \\ \mathrm{H} & 8.80143200 & -2.06850100 & -3.32492900 \\ \mathrm{C} & 10.39672900 & -1.78346900 & -4.77096300 \\ \mathrm{H} & 10.96198800 & -0.96697200 & -5.23734700 \\ \mathrm{H} & 11.11320500 & -2.43859100 & -4.26296200 \\ \mathrm{H} & 9.92542800 & -2.36237900 & -5.57473600 \\ \mathrm{C} & 10.11530100 & -1.72695500 & -0.16809900 \\ \mathrm{H} & 9.86810000 & -2.38047200 & 0.67753700 \\ \mathrm{H} & 9.91521200 & -2.30601600 & -1.07690700 \\ \mathrm{C} & 11.59071400 & -1.32355600 & -0.11583500 \\ \mathrm{H} & 11.81353400 & -0.74775900 & 0.79099200 \\ \mathrm{H} & 12.24411100 & -2.20296200 & -0.12308900 \\ \mathrm{H} & 11.85903100 & -0.70018200 & -0.97813300\end{array}$

\section{Figure-eight dimer 2 GS}

\begin{tabular}{|c|c|c|c|}
\hline & & & \\
\hline C & 9.33444900 & 2.62504300 & -0.90605900 \\
\hline C & 9.21902100 & 1.32311200 & -0.42948200 \\
\hline C & 10.31945300 & 0.51005000 & -0.15759300 \\
\hline C & 11.61008400 & 1.08380300 & -0.31596500 \\
\hline C & 11.72761700 & 2.42013000 & -0.73575300 \\
\hline C & 10.18462600 & -0.85497600 & 0.31538300 \\
\hline C & 11.34486500 & -1.61655300 & 0.61862700 \\
\hline C & 12.64254800 & -1.00829600 & 0.44252600 \\
\hline C & 12.76926900 & 0.28441900 & 0.00299200 \\
\hline C & 8.94760000 & -1.48637000 & 0.45770200 \\
\hline C & 8.80639400 & -2.78396100 & 0.93908100 \\
\hline C & 11.20770900 & -2.94891800 & 1.04364400 \\
\hline C & 10.62218500 & 3.21146700 & -1.07807200 \\
\hline C & 8.14131000 & 3.41251300 & -1.18483000 \\
\hline C & 6.91595500 & 2.78488700 & -1.39214000 \\
\hline C & 5.71989900 & 3.48390400 & -1.58059500 \\
\hline C & 5.80603600 & 4.90409300 & -1.62348500 \\
\hline C & 7.05770700 & 5.53363300 & -1.50550100 \\
\hline C & 4.43483900 & 2.82657200 & -1.73200100 \\
\hline C & 3.25828600 & 3.62015200 & -1.86624700 \\
\hline C & 3.37789800 & 5.05922200 & -1.89083700 \\
\hline 0 & 4.59961700 & 5.67264600 & -1.79621900 \\
\hline $\mathrm{C}$ & 4.28603200 & 1.42071600 & -1.72298400 \\
\hline $\mathrm{C}$ & 3.04932600 & 0.82385700 & -1.81783800 \\
\hline C & 1.99419400 & 3.00140300 & -1.95205700 \\
\hline C & 8.24253700 & 4.83510500 & -1.24526300 \\
\hline C & 9.96375000 & -3.55958700 & 1.25275100 \\
\hline $\mathrm{C}$ & 7.48131300 & -3.36991500 & 1.10305400 \\
\hline $\mathrm{C}$ & 6.34662100 & -2.56612900 & 1.19479400 \\
\hline C & 5.05353700 & -3.08066300 & 1.34636500 \\
\hline$C$ & 4.93274800 & -4.49480400 & 1.47500400 \\
\hline 0 & 6.08207300 & -5.30106300 & 1.42561800 \\
\hline C & 3.86815400 & -2.24264400 & 1.40477900 \\
\hline C & 2.59578400 & -2.85038300 & 1.61888400 \\
\hline C & 2.51243000 & -4.28505600 & 1.76330400 \\
\hline C & 3.62919900 & -5.07408900 & 1.68361800 \\
\hline C & 3.91027900 & -0.83668200 & 1.26114500 \\
\hline $\mathrm{C}$ & 2.77320400 & -0.06216200 & 1.34356100 \\
\hline $\mathrm{C}$ & 1.43796300 & -2.05218200 & 1.69584000 \\
\hline C & 7.36243700 & -4.78705000 & 1.20172000 \\
\hline & 8.57912900 & -5.62590100 & 1.24554200 \\
\hline & 9.80899100 & -4.99066000 & 1.60477800 \\
\hline & 8.56894200 & -7.03228800 & 1.02165100 \\
\hline & 9.67360000 & -7.79061300 & 1.44050600 \\
\hline
\end{tabular}

$10.72156600 \quad-7.17838800 \quad 2.16717000$ $\begin{array}{lll}10.83372600 & -5.77903100 & 2.20307600\end{array}$ $\begin{array}{llll}10.72272400 & 4.64533500 & -1.43482600\end{array}$ $9.57962900 \quad 5.47287900 \quad-1.18795100$ $\begin{array}{llll}11.91131600 & 5.24728600 & -1.93748400\end{array}$ $\begin{array}{llll}12.02664100 & 6.64617600 & -1.91551100\end{array}$ $\begin{array}{llll}11.02941400 & 7.43046900 & -1.29133900\end{array}$ $\begin{array}{llll}9.78315200 & 6.86704700 & -0.97188200\end{array}$ $\begin{array}{lll}-9.36067700 & 2.60268200 & 0.80481800\end{array}$ $\begin{array}{lll}-9.24966200 & 1.32175300 & 0.27748000\end{array}$ $\begin{array}{lll}-10.34786100 & 0.49704900 & 0.04191600\end{array}$ $\begin{array}{lll}-11.63764900 & 1.04565200 & 0.27366400\end{array}$ $\begin{array}{lll}-11.75520500 & 2.37114700 & 0.73028300\end{array}$ $\begin{array}{lll}-10.20688800 & -0.86327800 & -0.43916200\end{array}$ $\begin{array}{lll}-11.36302800 & -1.65264400 & -0.67719500\end{array}$ $\begin{array}{lll}-12.66233900 & -1.06552100 & -0.44606200\end{array}$ $\begin{array}{lll}-12.79438000 & 0.22536400 & 0.00025600\end{array}$ $\begin{array}{lll}-8.96338200 & -1.46883800 & -0.62584000\end{array}$ $-8.81455800-2.77589800-1.07657000$ $\begin{array}{lll}-11.21461400 & -2.99213000 & -1.07683200\end{array}$ $\begin{array}{lll}-10.64611800 & 3.16873700 & 1.05080000\end{array}$ $\begin{array}{lll}-8.16163700 & 3.37706400 & 1.08825500\end{array}$ $\begin{array}{lll}-6.93264500 & 2.74171500 & 1.23384800\end{array}$ $\begin{array}{lll}-5.73910400 & 3.42993900 & 1.46438900\end{array}$ $\begin{array}{lll}-5.82905100 & 4.84087100 & 1.63417600\end{array}$ $\begin{array}{lll}-7.08244800 & 5.47494300 & 1.55303800\end{array}$ $\begin{array}{lll}-4.45339000 & 2.76237700 & 1.53811600\end{array}$ $\begin{array}{lll}-3.28108100 & 3.53293100 & 1.78699000\end{array}$ $\begin{array}{lll}-3.40759000 & 4.95905800 & 1.98147600\end{array}$ $\begin{array}{lll}-4.62523600 & 5.58546800 & 1.90653000\end{array}$ $\begin{array}{lll}-4.30584400 & 1.36773400 & 1.36158300\end{array}$ $\begin{array}{lll}-3.07473100 & 0.75753800 & 1.42709300\end{array}$ $\begin{array}{lll}-2.02044900 & 2.90186700 & 1.83751200\end{array}$ $\begin{array}{lll}-8.26226800 & 4.79023000 & 1.23913300\end{array}$ $\begin{array}{lll}-9.96570200 & -3.58331300 & -1.31549000\end{array}$ $\begin{array}{lll}-7.48412400 & -3.34493900 & -1.25003500\end{array}$ $\begin{array}{lll}-6.37351400 & -2.51802000 & -1.41096200\end{array}$ $\begin{array}{lll}-5.06441900 & -3.00333800 & -1.50751500\end{array}$ $-4.90306400-4.41895300-1.48933300$ $\begin{array}{lll}-6.03182300 & -5.25245400 & -1.40932400\end{array}$ $-3.90136000-2.14108100-1.63915000$ $\begin{array}{lll}-2.60684600 & -2.73387700 & -1.71911900\end{array}$ $-2.47720400 \quad-4.17091100 \quad-1.67383600$ $\begin{array}{lll}-3.57603700 & -4.97868600 & -1.56507200\end{array}$ $\begin{array}{lll}-3.98057900 & -0.72969100 & -1.67838300\end{array}$ $\begin{array}{llll}-2.85489200 & 0.06037800 & -1.78289100\end{array}$ $\begin{array}{lll}-1.46319300 & -1.92063200 & -1.82524900\end{array}$ $-7.33429700 \quad-4.76427500 \quad-1.25543500$ $\begin{array}{lll}-1.56677800 & -0.52830300 & -1.85175400\end{array}$ $\begin{array}{llll}-8.54108400 & -5.62600100 & -1.25821300\end{array}$ $\begin{array}{lll}-9.78905300 & -5.02216000 & -1.61907400\end{array}$ $\begin{array}{lll}-8.51453700 & -7.02617700 & -0.99226400\end{array}$ $\begin{array}{lll}-9.61732200 & -7.81108100 & -1.36655000\end{array}$ $\begin{array}{lll}-10.68788900 & -7.23681200 & -2.08974500\end{array}$ $-10.81568500 \quad-5.84117200-2.16955300$ $\begin{array}{lll}-10.73878900 & 4.59006900 & 1.46205500\end{array}$ $\begin{array}{lll}-9.59888500 & 5.42590800 & 1.22677900\end{array}$ $\begin{array}{lll}-11.91428400 & 5.17966000 & 2.00914000\end{array}$ $\begin{array}{lll}-12.02412500 & 6.57932700 & 2.03737400\end{array}$ $\begin{array}{lll}-11.03615700 & 7.38259700 & 1.42178100\end{array}$ $\begin{array}{lll}-9.79770100 & 6.82651700 & 1.06202000\end{array}$ $\begin{array}{lll}1.87214800 & 1.61154400 & -1.91811800\end{array}$ $\begin{array}{lll}0.61510700 & 0.94897900 & -1.94856900\end{array}$ $\begin{array}{llll}-0.39811200 & 0.27698800 & -1.92203000\end{array}$ $\begin{array}{lll}1.50862500 & -0.66303500 & 1.56652900\end{array}$ $\begin{array}{lll}-1.90209100 & 1.52233700 & 1.65985000\end{array}$ $\begin{array}{lll}0.33784600 & 0.13915300 & 1.63704500\end{array}$ 


\begin{tabular}{|c|c|c|c|}
\hline C & -0.65400600 & 0.84167600 & 1.67629100 \\
\hline $\mathrm{H}$ & 8.22932400 & 0.94366700 & -0.21761800 \\
\hline $\mathrm{H}$ & 12.72238500 & 2.83059100 & -0.74544200 \\
\hline$H$ & 8.05648800 & -0.96029000 & 0.14389900 \\
\hline $\mathrm{H}$ & 12.12127500 & -3.50283400 & 1.16805200 \\
\hline $\mathrm{H}$ & 6.89612400 & 1.70431400 & -1.42436400 \\
\hline $\mathrm{H}$ & 7.06431000 & 6.59865700 & -1.65147800 \\
\hline $\mathrm{H}$ & 5.15767800 & 0.78217000 & -1.62999100 \\
\hline $\mathrm{H}$ & 2.95791700 & -0.25622300 & -1.78692400 \\
\hline 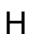 & 1.11130900 & 3.62308900 & -2.04215200 \\
\hline 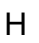 & 6.47931900 & -1.49261500 & 1.19402200 \\
\hline 11 & 5.94377000 & -6.34941400 & 1.62482200 \\
\hline 11 & 4.85378000 & -0.33883400 & 1.06652600 \\
\hline $\mathrm{H}$ & 2.83285100 & 1.01258000 & 1.21236700 \\
\hline H & 0.47970200 & -2.53032200 & 1.86066100 \\
\hline H & -8.26568600 & 0.96369000 & 0.01118300 \\
\hline H & -12.75475700 & 2.76411000 & 0.79447800 \\
\hline & -8.07132200 & -0.92016000 & -0.35766700 \\
\hline & -12.11961500 & -3.56952200 & -1.14779500 \\
\hline & -6.90856100 & 1.66186400 & 1.19941700 \\
\hline & -7.10552000 & 6.52435300 & 1.79073000 \\
\hline & -5.17157100 & 0.74924200 & 1.15312400 \\
\hline $\mathrm{H}$ & -2.98356000 & -0.31134800 & 1.26948200 \\
\hline $\mathrm{H}$ & -1.13748300 & 3.50682800 & 2.00646200 \\
\hline $\mathrm{H}$ & -6.53914200 & -1.45167500 & -1.48935600 \\
\hline $\mathrm{H}$ & -5.84425600 & -6.30681300 & -1.50297000 \\
\hline $\mathrm{H}$ & -4.94562300 & -0.23912200 & -1.61194400 \\
\hline $\mathrm{H}$ & -2.94385900 & 1.14137100 & -1.78697500 \\
\hline $\mathrm{H}$ & -0.48868600 & -2.39006000 & -1.88930000 \\
\hline $\mathrm{O}$ & -1.21454600 & -4.71640400 & -1.75839800 \\
\hline $\mathrm{O}$ & -3.45062000 & -6.34603400 & -1.45524100 \\
\hline C & -3.01289100 & -7.01904200 & -2.64238000 \\
\hline $\mathrm{H}$ & -3.02757500 & -8.08506900 & -2.40378800 \\
\hline $\mathrm{H}$ & -3.70153000 & -6.82277900 & -3.47445500 \\
\hline $\mathrm{H}$ & -2.00109600 & -6.70768300 & -2.91861800 \\
\hline C & -0.61466500 & -4.98785700 & -0.48235400 \\
\hline $\mathrm{H}$ & -0.53142800 & 900 & 0.11716200 \\
\hline $\mathrm{H}$ & -1.19352300 & -5.73946800 & 0.06720800 \\
\hline $\mathrm{H}$ & 0.38785500 & -5.36764300 & -0.68604700 \\
\hline C & 600 & 200 & 800 \\
\hline $\mathrm{H}$ & 74400 & 409300 & 70800 \\
\hline $\mathrm{H}$ & -7.79100400 & -8.25474400 & 0.60514700 \\
\hline C & -12.00289800 & -5.24486900 & -2.91515900 \\
\hline $\mathrm{H}$ & -11.81360400 & -4.19138200 & 0100 \\
\hline $\mathrm{H}$ & -12.07319700 & -5.72470200 & -3.9 \\
\hline $\mathrm{O}$ & -13.76874000 & -1.84137500 & -0.72617300 \\
\hline $\mathrm{O}$ & -14.03602000 & 0.77100800 & 0.25423000 \\
\hline C & -14.50549400 & -2.27718800 & 0.42375800 \\
\hline $\mathrm{H}$ & -13.86058200 & -2.85355900 & 1.09920600 \\
\hline $\mathrm{H}$ & -14.93342500 & -1.42503600 & 0.9618370 \\
\hline $\mathrm{H}$ & -15.30256800 & -2.92059900 & 0.0441170 \\
\hline C & -14.82572700 & 1.04240300 & -0.9110300 \\
\hline $\mathrm{H}$ & -15.06350000 & 0.11845300 & -1.4478390 \\
\hline $\mathrm{H}$ & -15.74276200 & 1.51349200 & -0.54961500 \\
\hline $\mathrm{H}$ & -14.29910000 & 1.73338700 & -1.58153700 \\
\hline C & -13.01406500 & 4.33565100 & 2.64039500 \\
\hline $\mathrm{H}$ & -12.65413800 & 3.31756800 & 2.80278300 \\
\hline $\mathrm{H}$ & -13.21727500 & 4.72359200 & 3.64755700 \\
\hline C & -8.76508300 & 7.71153600 & 0.36965000 \\
\hline $\mathrm{H}$ & -7.99847900 & 7.09324600 & -0.10444900 \\
\hline $\mathrm{H}$ & -9.27582300 & 8.21392200 & -0.46381800 \\
\hline $\mathrm{O}$ & -4.74836700 & 6.94474200 & 2.10744600 \\
\hline O & -2.25030500 & 5.67290000 & 2.21458200 \\
\hline C & -2.13176300 & 6.18546400 & 3.54906200 \\
\hline $\mathrm{H}$ & -2.92910400 & 6.90359900 & 3.76678600 \\
\hline & -2.16216800 & 5.36782900 & 4.28038800 \\
\hline & -115935800 & 668133600 & 35981770 \\
\hline
\end{tabular}

\begin{tabular}{|c|c|c|c|}
\hline C & -4.17328400 & 7.75404600 & 3700 \\
\hline L & -4.41542100 & 8.78807700 & 1.32753000 \\
\hline $\mathrm{H}$ & -3.08782500 & 7.62097800 & 1.02610700 \\
\hline $\mathrm{H}$ & -4.61572700 & 7.50650800 & 0.09972800 \\
\hline $\mathrm{O}$ & 2.21989700 & 5.79323800 & -2.03467000 \\
\hline 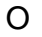 & 4.71423900 & 7.04701700 & -1.78834600 \\
\hline$\checkmark$ & 1.82014500 & 6.50183600 & -0.85279700 \\
\hline$\Pi$ & 1.65047100 & 5.80437100 & -0.02266900 \\
\hline $\mathrm{H}$ & 2.57474400 & 7.24170500 & -0.56570900 \\
\hline $\mathrm{H}$ & 0.88171400 & 7.00235600 & -1.10222200 \\
\hline C & 4.41561500 & 7.68886000 & -3.03496200 \\
\hline $\mathrm{H}$ & 4.60874900 & 8.75242000 & -2.87737900 \\
\hline$\Pi$ & 5.07213500 & 7.31150600 & -3.82920900 \\
\hline $\mathrm{H}$ & 3.36963000 & 7.53279400 & -3.31664600 \\
\hline C & 8.72247100 & 7.74423600 & -0.31738200 \\
\hline $\mathrm{H}$ & 7.95115500 & 7.11931300 & 0.13783800 \\
\hline $\mathrm{H}$ & 9.18452500 & 8.27407000 & 0.52638200 \\
\hline C & 13.01964100 & 4.42004000 & -2.57666000 \\
\hline r & 12.66241600 & 3.40632200 & -2.76962000 \\
\hline $\mathrm{H}$ & 13.23523600 & 4.83451900 & -3.57073500 \\
\hline 0 & 13.74898400 & -1.76187600 & 0.77715300 \\
\hline $\mathrm{O}$ & 14.01041900 & 0.85498400 & -0.19113500 \\
\hline C & 14.55336000 & -2.17916700 & -0.33362600 \\
\hline $\mathrm{H}$ & 13.95677800 & -2.76766800 & -1.04220500 \\
\hline $\mathrm{H}$ & 14.99025100 & -1.31685400 & -0.84762900 \\
\hline $\mathrm{H}$ & 15.34321300 & -2.80628600 & 0.08626300 \\
\hline $\mathrm{C}$ & 14.73176500 & 1.15045900 & 1.01210900 \\
\hline $\mathrm{H}$ & 14.15729100 & 1.83686400 & 1.64708600 \\
\hline $\mathrm{H}$ & 14.95726200 & 0.23526100 & 1.56895900 \\
\hline $\mathrm{H}$ & 15.65818600 & 1.63555700 & 0.69602800 \\
\hline C & 7.46970200 & -7.70993600 & 0.20778600 \\
\hline $\mathrm{H}$ & 6.84742900 & -6.95550200 & -0.28081100 \\
\hline $\mathrm{H}$ & 7.95998900 & -8.24759300 & -0.61617100 \\
\hline C & 11.99885300 & -5.14619500 & 2.95318700 \\
\hline $\mathrm{H}$ & 11.79152600 & -4.08985100 & 3.13761700 \\
\hline $\mathrm{H}$ & 12.05779700 & -5.59808600 & 3.95242300 \\
\hline $\mathrm{O}$ & 1.25972900 & -4.83420400 & 1.95129300 \\
\hline $\mathrm{O}$ & 3.55874800 & -6.44347000 & 1.83259700 \\
\hline $\mathrm{C}$ & 2.87634000 & -7.12920300 & 0.77512300 \\
\hline $\mathrm{H}$ & 2.99971900 & -8.19538100 & 0.97576200 \\
\hline $\mathrm{H}$ & 1.81298100 & -6.87045000 & 0.75767100 \\
\hline $\mathrm{H}$ & 3.32829600 & -6.88797100 & -0.19538800 \\
\hline C & 1.04098400 & $-5.38 s$ & 38900 \\
\hline $\mathrm{H}$ & 1.72236300 & -6.22522700 & 3.44539900 \\
\hline $\mathrm{H}$ & 1.17533800 & -4.62162100 & 4.02819200 \\
\hline $\mathrm{H}$ & 0.00637700 & -5.74000400 & 3.26569900 \\
\hline C & 9.77659200 & -9.27789800 & 1.16057900 \\
\hline $\mathrm{H}$ & 9.47311800 & -9.88208400 & 2.02648600 \\
\hline $\mathrm{H}$ & 9.15659200 & -9.59056800 & 0.31911100 \\
\hline $\mathrm{H}$ & 10.80650400 & -9.56291400 & 0.92325500 \\
\hline C & 11.73310000 & -8.07760200 & 2.85118000 \\
\hline $\mathrm{H}$ & 11.24740800 & -8.95557800 & 3.28871800 \\
\hline $\mathrm{H}$ & 12.49173300 & -8.45167300 & 2.15018700 \\
\hline $\mathrm{H}$ & 12.26459600 & -7.57080000 & 3.65790400 \\
\hline C & 6.55588900 & -8.71389700 & 0.94156900 \\
\hline $\mathrm{H}$ & 5.94587300 & -8.19887500 & 1.69250600 \\
\hline $\mathrm{H}$ & 7.16036300 & -9.43744600 & 1.49835200 \\
\hline C & 5.63702300 & -9.45621800 & -0.03405600 \\
\hline $\mathrm{H}$ & 4.95875800 & -10.13701600 & 0.49332800 \\
\hline $\mathrm{H}$ & 5.02456400 & -8.75302200 & -0.61113800 \\
\hline $\mathrm{H}$ & 6.21729000 & -10.05122900 & -0.75037200 \\
\hline C & 13.38373200 & -5.28893700 & 2.28157600 \\
\hline $\mathrm{H}$ & 13.71044300 & -6.33345900 & 2.33250300 \\
\hline $\mathrm{H}$ & 13.30529900 & -5.06087200 & 1.21100200 \\
\hline C & 14.43513700 & -4.37977500 & 2.92472400 \\
\hline $\mathrm{H}$ & 14.18281100 & -3.32511700 & 2.76911000 \\
\hline $\mathrm{H}$ & 14.50604000 & -4.56103400 & 4.00464500 \\
\hline
\end{tabular}




\begin{tabular}{|c|c|c|c|}
\hline $\mathrm{H}$ & 15.42792400 & -4.55377400 & 2.49254800 \\
\hline C & 14.34786800 & 4.35483300 & -1.78854300 \\
\hline $\mathrm{H}$ & 14.84485200 & 5.33049700 & -1.82500500 \\
\hline H & 14.14424800 & 4.17228700 & -0.72572100 \\
\hline C & 15.28542800 & 3.26918500 & -2.32503400 \\
\hline $\mathrm{H}$ & 14.85339200 & 2.27429900 & -2.17141400 \\
\hline & 15.47023800 & 3.40261200 & -3.39843300 \\
\hline & 16.25577700 & 3.29484300 & -1.81474200 \\
\hline & 8.05835500 & 8.79680800 & -1.23311300 \\
\hline & 8.79219700 & 9.56463500 & -1.50103300 \\
\hline & 7.76213300 & 8.33419200 & -2.18304400 \\
\hline & 6.83745300 & 9.44515100 & -0.57403500 \\
\hline & 7.10077600 & 9.88804600 & 0.39483300 \\
\hline & 6.42384200 & 10.24300900 & -1.20256900 \\
\hline & 6.04814500 & 8.70361900 & -0.40953300 \\
\hline & 13.22589500 & 7.35784800 & -2.51155100 \\
\hline & 13.97114300 & 7.61931400 & -1.74805900 \\
\hline & 13.73636500 & 6.75512300 & -3.26410300 \\
\hline & 12.92791900 & 8.29304200 & -2.99593600 \\
\hline & 11.33911400 & 8.89090200 & -1.02232400 \\
\hline & 11.20206800 & 9.51173500 & -1.91820700 \\
\hline & 10.70920500 & 9.31515800 & -0.23923400 \\
\hline & 12.37818500 & 9.02139100 & -0.70384800 \\
\hline & -8.07863800 & 8.80005900 & 1.22130600 \\
\hline & -8.82960600 & 9.37454200 & 1.77378300 \\
\hline & -7.43756400 & 8.34204500 & 1.98328100 \\
\hline & -7.24175700 & 9.74760500 & 0.35622800 \\
\hline & -6.48343200 & 9.19585600 & -0.21204500 \\
\hline & -7.87083900 & 10.28129700 & -0.36721300 \\
\hline & -6.72444700 & 10.49693300 & 0.96650400 \\
\hline & -14.35150300 & 4.29169200 & 1.86700600 \\
\hline & -14.84262000 & 5.26918500 & 1.92834000 \\
\hline & -14.16106300 & 4.12866000 & 0.79853100 \\
\hline & -15.28813200 & 3.20042100 & 2.39380800 \\
\hline & -14.86104400 & 2.20657900 & 2.22022800 \\
\hline & -15.46268000 & 3.31648000 & 3.47093800 \\
\hline & -16.26301700 & 3.23816100 & 1.89298000 \\
\hline & -11.35213000 & 8.84925200 & 1.19665600 \\
\hline & -11.21280600 & 9.44542000 & 2.10889200 \\
\hline & -10.72984400 & 9.29945100 & 0.42194100 \\
\hline & -12.39409600 & 8.98415100 & 0.88926400 \\
\hline & -13.21335800 & 7.27381100 & 2.67272800 \\
\hline & -13.96731100 & 7.56220300 & 1.92768100 \\
\hline & -13.71655300 & 6.64825400 & 3.41131300 \\
\hline & -12.90640300 & 8.19179100 & 3.18372900 \\
\hline & -6.46723500 & -8.62942700 & -1.05162800 \\
\hline & -7.03540800 & -9.52247800 & -1.33395000 \\
\hline & -6.18604400 & -8.15308700 & -1.99932100 \\
\hline & -5.20450900 & -9.03246000 & -0.28423200 \\
\hline & -5.45834800 & -9.48519000 & 0.68266600 \\
\hline & -4.61328100 & -9.76397600 & -0.84828400 \\
\hline & -4.57035500 & -8.15878800 & -0.09886800 \\
\hline & -13.37401000 & -5.38731200 & -2.21530700 \\
\hline & -13.69466100 & -6.43451900 & -2.24489100 \\
\hline & -13.27722800 & -5.14345700 & -1.14968100 \\
\hline & -14.44399200 & -4.49454800 & -2.85077100 \\
\hline & -14.53235200 & -4.68951600 & -3.92700600 \\
\hline & -15.42757700 & -4.67098000 & -2.39897900 \\
\hline & -14.19799100 & -3.43601000 & -2.71220600 \\
\hline & -11.70076100 & -8.16881700 & -2.72619000 \\
\hline & -12.44063100 & -8.53051700 & -1.99916500 \\
\hline & -12.25411500 & -7.69300700 & -3.53701300 \\
\hline & -11.21244300 & -9.05371300 & -3.14646700 \\
\hline & -9.68833100 & -9.29291500 & -1.05032100 \\
\hline & -9.39184400 & -9.91064300 & -1.90895700 \\
\hline & -9.04503400 & -9.57481900 & -0.21583600 \\
\hline & & 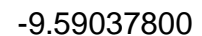 & \\
\hline
\end{tabular}

\section{Figure-eight dimer 2 TS}

$\begin{array}{llll}\text { C } & 9.49814900 & 3.06019100 & 0.09049600\end{array}$ C $\quad 9.52157600 \quad 1.66084500 \quad 0.13913600$ $\begin{array}{llll}\text { C } & 10.69438900 & 0.91901700 & 0.33594300\end{array}$ C $\quad 11.90420900 \quad 1.64784700 \quad 0.54871200$ $\begin{array}{llll}\text { C } & 11.89030600 & 3.04293500 & 0.45113000\end{array}$ C $\quad 10.75009100 \quad-0.54212300 \quad 0.31631200$ C $\quad 11.99714200-1.18365800 \quad 0.58338600$ $\begin{array}{llll}\text { C } & 13.17277500 & -0.40264100 & 0.85692300\end{array}$ $\begin{array}{llll}\text { C } & 13.13140500 & 0.95216700 & 0.82623800\end{array}$ C $\quad 9.65495000-1.36109000 \quad 0.01034600$ C $\quad 9.72584400 \quad-2.75962800 \quad-0.04236100$ $\begin{array}{llll}\text { C } & 12.07175900 & -2.57838100 & 0.53671800\end{array}$ $\begin{array}{llll}\text { C } & 10.73338500 & 3.77394400 & 0.18476300\end{array}$ C $\quad 8.24535300 \quad 3.80816200-0.08280000$ $\begin{array}{llll}\text { C } & 6.99313600 & 3.20697000 & 0.09471000\end{array}$ C $\quad 5.78006700 \quad 3.88390900-0.10718400$ C $\quad 5.85500900 \quad 5.23568900-0.56703700$ C $\quad 7.10494300 \quad 5.85131800-0.69355300$ $\begin{array}{llll}\text { C } & 4.46618800 & 3.30034600 & 0.14559700\end{array}$ C $\quad 3.30646900 \quad 4.08403900-0.13723600$ C $\quad 3.43602200 \quad 5.41466100-0.66675200$ C $\quad 4.65697400 \quad 5.97066000 \quad-0.85978000$ $\begin{array}{llll}\text { C } & 4.26087400 & 2.01184100 & 0.69491300\end{array}$ $\begin{array}{llll}\text { C } & 2.99461800 & 1.52239900 & 0.97384000\end{array}$ $\begin{array}{llll}\text { C } & 2.03090800 & 3.56760400 & 0.13288600\end{array}$ C $\quad 8.30413200 \quad 5.19693200-0.42166300$ C $\quad 10.98141500 \quad-3.39090700 \quad 0.22728300$ C $\quad 8.55710400 \quad-3.58707700 \quad-0.38411000$ C $\quad 7.28207000-3.03527400 \quad-0.56754700$ C $\quad 6.14839600 \quad-3.79558100-0.89369800$ C $\quad 6.32850000 \quad-5.20471200 \quad-1.04975100$ C $\quad 7.59898200-5.75845900-0.86709200$ C $\quad 4.80665300-3.23833200-1.03880300$ C $\quad 3.71980600-4.13364400 \quad-1.27717200$ C $\quad 3.95845500 \quad-5.54128400 \quad-1.44940600$ C $\quad 5.20977100-6.05253800-1.35124400$ C $\quad 4.50060300-1.85990400-0.93620300$ C $\quad 3.20031500-1.38760700 \quad-0.99607800$ C $\quad 2.40620100-3.64309300-1.31816500$ C $\quad 8.71884200-5.00273300-0.52656600$ C $\quad 10.01944800 \quad-5.63899600 \quad-0.27691100$ $\begin{array}{llll}11.12148900 & -4.85041000 & 0.13773700\end{array}$ C $\quad 10.20115800-7.03256200-0.41293400$ C $\quad 11.41111900-7.64320600 \quad-0.13149900$ C $\quad 12.49083800-6.867782000 .30740000$ $\begin{array}{llll}\text { C } & 12.34043600 & -5.49768700 & 0.43527700\end{array}$ $\begin{array}{llrr}\text { C } & 12.34043600 & -5.49768700 & 0.43527700 \\ \text { C } & 10.77370000 & 5.22382500 & -0.04908100\end{array}$ C $\quad 9.58935600 \quad 5.90907800 \quad-0.42231400$ C $\quad 11.97802300 \quad 5.95462800 \quad 0.03482300$ C $\quad 12.03413000 \quad 7.30646300 \quad-0.25931300$ $\begin{array}{llll}\text { C } & 10.87107400 & 7.97610800 & -0.65767300\end{array}$ C $\quad 9.67451300 \quad 7.28338000 \quad-0.73184900$ C $\quad-9.47445500 \quad-3.07412700 \quad-0.04412700$ $\begin{array}{llll}C & -9.47445500 & -3.07412700 & -0.04412700 \\ \text { C } & -9.49791500 & -1.67478400 & -0.09282500\end{array}$ C $\quad-10.67075700 \quad-0.93298900-0.28958300$ C $\quad-11.88058500-1.66184800-0.50219100$ C $\quad-11.86664400-3.05693200-0.40456000$ $\begin{array}{llll}\text { C } & -10.72647600 & 0.52815000 & -0.27004400\end{array}$ C $\quad-11.97356000 \quad 1.16965400-0.53703600$ $\begin{array}{llll}\text { C } & -13.14921700 & 0.38860300 & -0.81038600\end{array}$ C $\quad-13.10782500-0.96620200-0.77961700$ $\begin{array}{llll}\text { C } & -9.63131600 & 1.34714900 & 0.03576000\end{array}$ $\begin{array}{llll}\text { C } & -9.70221800 & 2.74568900 & 0.08837600\end{array}$ C $\quad-12.04818100 \quad 2.56438300 \quad-0.49048400$ C $\quad-10.70967900 \quad-3.78791300 \quad-0.13830400$ 
$\mathrm{H}$

$\mathrm{H}$

$\mathrm{H}$ $\begin{array}{lll}-5.09113100 & -1.40006600 & -0.88898700\end{array}$ $\begin{array}{lll}-2.85330000 & -0.55220100 & -1.36972800\end{array}$ $\begin{array}{lll}-1.13488300 & -4.19084900 & 0.13148400\end{array}$ $\begin{array}{lll}-7.12789600 & 1.95734600 & 0.46534300\end{array}$ $\begin{array}{lll}-7.65602700 & 6.81984900 & 1.02187900\end{array}$ $\begin{array}{lll}-5.27577500 & 1.12348800 & 0.84573300\end{array}$ $\begin{array}{lll}-2.97997600 & 0.31179600 & 0.94860000\end{array}$ $\begin{array}{lll}-1.56337500 & 4.32810500 & 1.50798100\end{array}$ $2.83942400-6.38941100-1.72023600$ $\begin{array}{lll}5.44364900 & -7.45341200 & -1.51783200\end{array}$ $\begin{array}{lll}2.49727500 & -7.11891500 & -0.53885700\end{array}$ $\begin{array}{lll}1.44156900 & -7.29219400 & -0.51988100\end{array}$ $\begin{array}{lll}3.01271200 & -8.05658200 & -0.53686100\end{array}$ $\begin{array}{lll}2.78152900 & -6.55382000 & 0.32414300\end{array}$ $\begin{array}{lll}5.35128200 & -7.79122700 & -2.90428500\end{array}$ $\begin{array}{lll}6.18103900 & -8.40939600 & -3.17681400\end{array}$ $\begin{array}{llll}4.43855600 & -8.32048000 & -3.08239200\end{array}$ $\begin{array}{llll}5.36513900 & -6.89657700 & -3.49106300\end{array}$ $9.03536700-7.92061700-0.88610100$ $\begin{array}{lll}8.67286000 & -7.56202900 & -1.82679400\end{array}$ $9.37682000-8.92867300-0.99625700$ $\begin{array}{lll}13.54953800 & -4.67870000 & 0.92408200\end{array}$ $\begin{array}{lll}13.87085000 & -4.01807500 & 0.14611400\end{array}$ $\begin{array}{lll}13.26903200 & -4.10719700 & 1.78408200\end{array}$ $\begin{array}{lll}14.39566900 & -1.09078300 & 1.13240400\end{array}$ $\begin{array}{lll}14.31036700 & 1.72535600 & 1.06525200\end{array}$ $\begin{array}{lll}14.51175600 & -1.31132200 & 2.54051900\end{array}$ $\begin{array}{lll}15.09908700 & -2.18813700 & 2.71698200\end{array}$ $\begin{array}{lll}14.98567600 & -0.46697200 & 2.99590000\end{array}$ $\begin{array}{lll}13.53736800 & -1.44387700 & 2.96229800\end{array}$ $\begin{array}{lll}15.36658000 & 1.25838300 & 0.22189700\end{array}$ $\begin{array}{lll}15.93856300 & 2.09215500 & -0.12819000\end{array}$ $\begin{array}{lll}16.00059800 & 0.59858800 & 0.77650600\end{array}$ $\begin{array}{llll}14.95089400 & 0.73499200 & -0.61366700\end{array}$ $\begin{array}{lll}13.27959400 & 5.24844600 & 0.45769800\end{array}$ $\begin{array}{lll}13.12766000 & 4.75035200 & 1.39242800\end{array}$ $\begin{array}{lll}14.06048300 & 5.97246300 & 0.56215500\end{array}$ $\begin{array}{llll}8.41320100 & 8.05744800 & -1.15788800\end{array}$ $\begin{array}{lll}7.96338300 & 7.57148300 & -1.99836600\end{array}$ $\begin{array}{llll}7.71763600 & 8.08096000 & -0.34515300\end{array}$ $\begin{array}{llll}4.78276100 & 7.30708700 & -1.35277100\end{array}$ $\begin{array}{lll}2.24267200 & 6.14784900 & -0.95528700\end{array}$ $\begin{array}{llll}5.07298600 & 8.19055300 & -0.26642000\end{array}$ $\begin{array}{lll}5.75485400 & 8.94712800 & -0.59437100\end{array}$ $\begin{array}{lll}4.16741000 & 8.64904000 & 0.07214000\end{array}$ $\begin{array}{llll}5.51385400 & 7.63654600 & 0.53583300\end{array}$ $\begin{array}{lll}2.15177100 & 6.37513400 & -2.36417900\end{array}$ $\begin{array}{lll}1.14895000 & 6.19438700 & -2.69064600\end{array}$ $\begin{array}{lll}2.42001300 & 7.38821900 & -2.58006300\end{array}$ $\begin{array}{llll}2.81833100 & 5.71286500 & -2.87603500\end{array}$ $\begin{array}{lll}-2.81574100 & 6.37558100 & 1.76578200\end{array}$ $\begin{array}{lll}-5.41997100 & 7.43958000 & 1.56336100\end{array}$ $\begin{array}{lll}-2.33325700 & 6.12072200 & 3.08758200\end{array}$ $\begin{array}{lll}-1.36129400 & 5.67663700 & 3.03303200\end{array}$ $\begin{array}{lll}-2.27482000 & 7.04141100 & 3.62963300\end{array}$ $\begin{array}{lll}-3.00263800 & 5.45342200 & 3.58911900\end{array}$ $\begin{array}{lll}-6.04622600 & 7.96124500 & 0.38838500\end{array}$ $\begin{array}{llll}-7.04991000 & 8.25212100 & 0.61840700\end{array}$ $\begin{array}{llll}-5.49903100 & 8.81241700 & 0.04055700\end{array}$ $\begin{array}{lll}-6.05833400 & 7.20953500 & -0.37298600\end{array}$ $\begin{array}{lll}-9.01168500 & 7.90673300 & 0.93168000\end{array}$ $\begin{array}{lll}-8.59852800 & 7.50306200 & 1.83237500\end{array}$ $\begin{array}{lll}-8.25604700 & 7.93650500 & 0.17469500\end{array}$ $\begin{array}{lll}-13.52591100 & 4.66471800 & -0.87819800\end{array}$ $\begin{array}{lll}-13.24544500 & 4.09326200 & -1.73824200\end{array}$ $\begin{array}{llll}-13.84714600 & 4.00404900 & -0.10023600\end{array}$ $\begin{array}{lll}-14.28680200 & -1.73942300 & -1.01845000\end{array}$ 


\begin{tabular}{|c|c|c|c|}
\hline ) & -14.37215000 & 1.07671400 & -1.08577200 \\
\hline C & -14.42737300 & -1.97303900 & -2.42221800 \\
\hline & -14.62534800 & -3.01059400 & -2.59305400 \\
\hline $\mathrm{H}$ & -15.23887300 & -1.38790000 & -2.80166700 \\
\hline & -13.52308100 & -1.69542700 & -2.92230500 \\
\hline & -15.10282300 & 1.25544400 & 0.13039800 \\
\hline & -15.66260500 & 2.16592200 & 0.07965400 \\
\hline & -15.77238100 & 0.43232300 & 0.26847300 \\
\hline & -14.42021100 & 1.30182300 & 0.95307100 \\
\hline & -8.38928200 & -8.07138200 & 1.20410700 \\
\hline & -7.93919400 & -7.58517100 & 2.04429800 \\
\hline & -8.65831600 & -9.07154700 & 1.47279300 \\
\hline & -13.25578400 & -5.26256800 & -0.41147700 \\
\hline & -13.52237700 & -4.53445600 & 0.32587000 \\
\hline & -13.10869400 & -4.77821000 & -1.35416600 \\
\hline & -4.75895300 & -7.32068400 & 1.39972000 \\
\hline & -2.21889300 & -6.16143600 & 1.00206700 \\
\hline & -4.30289200 & -7.37386100 & 2.75400300 \\
\hline & -4.94384900 & -8.01597700 & 3.32124200 \\
\hline & -3.30341300 & -7.75506200 & 2.77906200 \\
\hline & -4.32016500 & -6.39033500 & 3.17504700 \\
\hline & -1.88009400 & -6.98131100 & -0.11950400 \\
\hline & -1.59850400 & -6.35966500 & -0.94361700 \\
\hline & -1.06269700 & -7.62104300 & 0.14031200 \\
\hline & -2.72557400 & -7.57669600 & -0.39442200 \\
\hline & -11.54477900 & 9.15193400 & 0.34565800 \\
\hline & -11.32533500 & 9.42339400 & 1.35711900 \\
\hline & -10.86879900 & 9.65545300 & -0.31344700 \\
\hline & -12.54950800 & 9.43489600 & 0.11038900 \\
\hline & -13.81344900 & 7.52067100 & -0.60008100 \\
\hline & -14.13263100 & 7.20679100 & -1.57193600 \\
\hline & -14.54572700 & 7.23424400 & 0.12561100 \\
\hline & -13.69737800 & 8.58430000 & -0.58903600 \\
\hline & -14.67634400 & 5.61904900 & -1.24881700 \\
\hline & -14.98070000 & 6.16497500 & -0.38035100 \\
\hline & -14.34396600 & 6.30291000 & -2.00165100 \\
\hline & -15.86710500 & 4.80340500 & -1.78585800 \\
\hline & -15.53070700 & 4.15208300 & -2.56529100 \\
\hline & -16.28816900 & 4.22218400 & -0.99226700 \\
\hline & -16.60978400 & 5.46923500 & -2.17315400 \\
\hline & -9.52805800 & 9.33294400 & 1.19791500 \\
\hline & -9.82484700 & 9.78313500 & 0.27371600 \\
\hline & -10.36769600 & 9.29050900 & 1.85981300 \\
\hline & -8.40882700 & 10.17229000 & 1.84167000 \\
\hline & -7.99369700 & 9.63758300 & 2.67031600 \\
\hline & -7.64286600 & 10.35996600 & 1.11849600 \\
\hline & -8.81227200 & 11.10250400 & 2.18348100 \\
\hline & -14.38537400 & -6.30150700 & -0.53888300 \\
\hline & -14.31940200 & -6.78514800 & -1.49105900 \\
\hline & -14.28912200 & -7.03019400 & 0.23871000 \\
\hline & -15.74844200 & -5.59560200 & -0.41502100 \\
\hline & -15.72096800 & -4.90825600 & 0.40455200 \\
\hline & -15.95824400 & -5.06390200 & -1.31955400 \\
\hline & -16.51318100 & -6.32418000 & -0.24400300 \\
\hline & -7.38850700 & -8.10571100 & 0.03411900 \\
\hline & -7.49059500 & -7.21427600 & -0.54882300 \\
\hline & -6.39202100 & -8.16811300 & 0.41885300 \\
\hline & -7.67499900 & -9.33322800 & -0.85059800 \\
\hline & -7.84442400 & -10.18834000 & -0.23013100 \\
\hline & -8.54356200 & -9.14785800 & -1.44737100 \\
\hline & -6.83606900 & -9.51637100 & -1.48899700 \\
\hline & -13.34377700 & -8.08281300 & 0.19503400 \\
\hline & -14.03628400 & -7.69596800 & 0.91314600 \\
\hline & -13.74459500 & -7.95949800 & -0.78936300 \\
\hline & -13.17700200 & -9.12260500 & 0.38455600 \\
\hline & -10.89819800 & -9.48816000 & 1.05733700 \\
\hline & & & \\
\hline
\end{tabular}

$\begin{array}{lrrr}\mathrm{H} & -10.15722600 & -9.70528600 & 1.79809200 \\ \mathrm{H} & -11.86728200 & -9.73146800 & 1.44019100 \\ \mathrm{C} & 7.89987600 & -7.87392500 & 0.15316800 \\ \mathrm{H} & 7.85843800 & -6.89921000 & 0.59261400 \\ \mathrm{H} & 6.96800100 & -8.08867300 & -0.32682500 \\ \mathrm{C} & 8.16522800 & -8.92101900 & 1.25079400 \\ \mathrm{H} & 8.45432000 & -9.84657000 & 0.79837800 \\ \mathrm{H} & 8.95056700 & -8.57605200 & 1.89043600 \\ \mathrm{H} & 7.27516600 & -9.06796000 & 1.82620500 \\ \mathrm{C} & 14.69991100 & -5.63305600 & 1.29482200 \\ \mathrm{H} & 14.52409000 & -6.04264300 & 2.26756300 \\ \mathrm{H} & 14.75080100 & -6.42574600 & 0.57791700 \\ \mathrm{C} & 16.03009000 & -4.85703000 & 1.29734800 \\ \mathrm{H} & 16.07476100 & -4.22300400 & 0.43658300 \\ \mathrm{H} & 16.09332300 & -4.26050700 & 2.18338500 \\ \mathrm{H} & 16.84640200 & -5.54839200 & 1.27383100 \\ \mathrm{C} & 11.56845000 & -9.16583800 & -0.30013800 \\ \mathrm{H} & 11.06151700 & -9.66731000 & 0.49763700 \\ \mathrm{H} & 11.14591500 & -9.46657600 & -1.23604400 \\ \mathrm{H} & 12.60723100 & -9.42155900 & -0.27917700 \\ \mathrm{C} & 13.83710500 & -7.53463300 & 0.64573800 \\ \mathrm{H} & 14.58670800 & -7.19089500 & -0.03605400 \\ \mathrm{H} & 14.12027600 & -7.27937400 & 1.64551700 \\ \mathrm{H} & 13.73972700 & -8.59696300 & 0.56283000 \\ \mathrm{C} & 13.67593800 & 4.21710500 & -0.61507700 \\ \mathrm{H} & 14.18408600 & 3.39752700 & -0.15141900 \\ \mathrm{H} & 12.79621000 & 3.85870700 & -1.10754800 \\ \mathrm{C} & 14.60708200 & 4.88117000 & -1.64638100 \\ \mathrm{H} & 14.04090000 & 5.55196700 & -2.25823800 \\ \mathrm{H} & 15.37419800 & 5.42522000 & -1.13604400 \\ \mathrm{H} & 15.05311300 & 4.12771900 & -2.26141700 \\ \mathrm{C} & 8.80038300 & 9.49710400 & -1.54399800 \\ \mathrm{H} & 9.53957800 & 9.86268400 & -0.86220500 \\ \mathrm{H} & 9.19664800 & 9.50550300 & -2.53788100 \\ \mathrm{C} & 7.55335000 & 10.39850900 & -1.48093100 \\ \mathrm{H} & 6.83570900 & 10.06544900 & -2.20132200 \\ \mathrm{H} & 7.12558800 & 10.34754600 & -0.50148100 \\ \mathrm{H} & 7.83230800 & 11.40883300 & -1.69617100 \\ \mathrm{C} & 10.92219200 & 9.47410100 & -1.01123500 \\ \mathrm{H} & 10.52977100 & 9.62181300 & -1.99565700 \\ \mathrm{H} & 10.33625200 & 10.02697300 & -0.30702900 \\ \mathrm{H} & 11.93607100 & 9.81432800 & -0.97667600 \\ \mathrm{C} & 13.36771900 & 8.06866600 & -0.14892700 \\ \mathrm{H} & 13.97234600 & 7.62120100 & 0.61206000 \\ \mathrm{H} & 13.88265600 & 8.02402200 & -1.08580900 \\ & 13.17474100 & 9.09036000 & 0.10366500\end{array}$


1-Benz GS (no D3)

\begin{tabular}{|c|c|c|c|}
\hline C & -3.84234200 & -0.70153600 & 0.10468600 \\
\hline C & -5.07841600 & -1.40454400 & 0.11171200 \\
\hline $\mathrm{C}$ & -3.83767600 & 0.73471700 & -0.12097700 \\
\hline $\mathrm{C}$ & -2.66408800 & 1.47337100 & -0.28642800 \\
\hline $\mathrm{C}$ & -2.65187900 & 2.85752800 & -0.45184800 \\
\hline $\mathrm{C}$ & -3.87997100 & 3.58262000 & -0.41593900 \\
\hline $\mathrm{C}$ & -5.05467200 & 2.84804500 & -0.22186400 \\
\hline $\mathrm{C}$ & -1.38144700 & 3.57766500 & -0.41676100 \\
\hline $\mathrm{C}$ & -1.40390400 & 4.94705400 & -0.01034400 \\
\hline $\mathrm{C}$ & -2.62548000 & 5.74529500 & -0.24656400 \\
\hline $\mathrm{C}$ & -3.85428800 & 5.05701600 & -0.51538100 \\
\hline $\mathrm{C}$ & -0.15909600 & 2.92476900 & -0.56618800 \\
\hline $\mathrm{C}$ & 1.04708700 & 3.47934200 & -0.11927100 \\
\hline $\mathrm{C}$ & -0.24228900 & 5.43283600 & 0.59683400 \\
\hline $\mathrm{C}$ & 2.32349000 & 2.79566100 & -0.23520100 \\
\hline $\mathrm{C}$ & -5.06888200 & 1.44619500 & -0.12770900 \\
\hline $\mathrm{C}$ & 0.96664500 & 4.71346100 & 0.59087700 \\
\hline $\mathrm{C}$ & 3.42635800 & 3.26749400 & 0.52978600 \\
\hline $\mathrm{C}$ & 4.63723500 & 2.54637300 & 0.52531200 \\
\hline $\mathrm{C}$ & 2.53461700 & 1.67844100 & -1.07347500 \\
\hline $\mathrm{C}$ & -6.31179300 & -0.66067200 & 0.01663200 \\
\hline C & 3.29590300 & 4.48504800 & 1.29148200 \\
\hline C & 3.74773800 & 1.01676200 & -1.10580600 \\
\hline C & -6.30723300 & 0.71069500 & -0.03245800 \\
\hline $\mathrm{C}$ & 2.12711300 & 5.19883200 & 1.29174300 \\
\hline $\mathrm{C}$ & 4.81296000 & 1.41784400 & -0.26778200 \\
\hline $\mathrm{H}$ & -1.71579400 & 0.95824400 & -0.22349700 \\
\hline $\mathrm{H}$ & -6.00440100 & 3.34597500 & -0.11620100 \\
\hline $\mathrm{H}$ & -0.15002800 & 1.92206500 & -0.97618100 \\
\hline $\mathrm{H}$ & -0.24447000 & 6.38170000 & 192400 \\
\hline $\mathrm{H}$ & 5.43892400 & 2.88790500 & 1.17032800 \\
\hline $\mathrm{H}$ & 1.73440300 & 1.33562700 & -1.72170000 \\
\hline $\mathrm{H}$ & 3.87547700 & 0.15633900 & -1.75433800 \\
\hline $\mathrm{C}$ & -5.00395000 & 5.80317500 & -0.88370300 \\
\hline $\mathrm{C}$ & -4.98844500 & 7.19314700 & 08900 \\
\hline $\mathrm{C}$ & -3.78532000 & 7.87467900 & 564300 \\
\hline C & -2.57864400 & 7.16620100 & -0.34739700 \\
\hline $\mathrm{C}$ & 6.09690800 & 0.65698600 & -0.17118600 \\
\hline $\mathrm{C}$ & 7.31560100 & 1.33066300 & -0.40833200 \\
\hline $\mathrm{C}$ & 8.51575300 & 0.63905100 & -0.19157100 \\
\hline $\mathrm{C}$ & 8.51525300 & -0.69539700 & 0.23401500 \\
\hline $\mathrm{C}$ & 7.31319600 & -1.38508200 & 0.45096400 \\
\hline $\mathrm{C}$ & 6.09597300 & -0.71014300 & 0.20936100 \\
\hline $\mathrm{C}$ & 9.84278600 & -1.33695600 & 0.51674400 \\
\hline $\mathrm{C}$ & 9.84130200 & 1.28377800 & -0.47850400 \\
\hline $\mathrm{O}$ & 10.68285700 & -0.87154900 & 1.25838600 \\
\hline $\mathrm{O}$ & 10.66316200 & 0.84186400 & -1.25363500 \\
\hline C & 7.34088000 & -2.79700300 & 1.00564000 \\
\hline $\mathrm{H}$ & 8.15924400 & -2.91652000 & 1.72182800 \\
\hline $\mathrm{H}$ & 6.40722300 & -3.04094800 & 1.51498200 \\
\hline $\mathrm{C}$ & 7.35116500 & 2.74546100 & -0.95580300 \\
\hline $\mathrm{H}$ & 6.41876800 & 2.99887000 & -1.46292600 \\
\hline $\mathrm{H}$ & 8.16938900 & 2.86364900 & -1.67263700 \\
\hline $\mathrm{C}$ & -2.67359300 & -1.44811800 & 0.26959400 \\
\hline $\mathrm{C}$ & -2.67075300 & 37600 & 32900 \\
\hline $\mathrm{C}$ & -3.90390200 & -3.54892100 & 0.39866300 \\
\hline $\mathrm{C}$ & -5.07372800 & -2.80644000 & 0.20562500 \\
\hline $\mathrm{C}$ & -1.40536800 & -3.56135200 & 0.39922800 \\
\hline $\mathrm{C}$ & -1.43810800 & -4.92970800 & -0.00957200 \\
\hline $\mathrm{C}$ & -2.66501200 & -5.71974400 & 0.22488300 \\
\hline $\mathrm{C}$ & -3.88842200 & -5.02350800 & 0.49696100 \\
\hline C & -0.17795800 & -2.91868100 & 0.55214200 \\
\hline C & 1.02482700 & -3.48544600 & 0.11114100 \\
\hline $\mathrm{C}$ & -0.28026500 & -5.42337700 & -0.61738700 \\
\hline 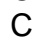 & 2.31096900 & -2.82685500 & 0.25147500 \\
\hline
\end{tabular}

$0.93602000-4.71717400 \quad-0.60241200$ $\begin{array}{llll}3.41435000 & -3.30583900 & -0.50931700\end{array}$ $\begin{array}{llll}2.52718500 & -1.71694000 & 1.09820200\end{array}$ $\begin{array}{lll}4.80966400 & -1.46804000 & 0.30228900\end{array}$ $\begin{array}{llll}4.63135900 & -2.59472400 & -0.49305200\end{array}$ $\begin{array}{lll}3.74440000 & -1.06365400 & 1.13856800\end{array}$ $\begin{array}{llll}3.27418200 & -4.51371800 & -1.28602700\end{array}$ $2.09817400 \quad-5.21858800 \quad-1.28840500$ $\begin{array}{lll}-1.72165100 & -0.93966300 & 0.20666100\end{array}$ $\begin{array}{lll}-6.02683500 & -3.29799100 & 0.10024600\end{array}$ $\begin{array}{lll}-0.16102300 & -1.91644600 & 0.96314200\end{array}$ $\begin{array}{lll}-0.29561800 & -6.36266600 & -1.14942600\end{array}$ $\begin{array}{lll}5.43993800 & -2.94796600 & -1.12253600\end{array}$ $\begin{array}{lll}-5.04231800 & -5.76205300 & 0.86727300\end{array}$ $\begin{array}{lll}-5.03677500 & -7.15197600 & 0.69594500\end{array}$ $\begin{array}{lll}-2.62810300 & -7.14119500 & 0.32320600\end{array}$ $\begin{array}{lll}-3.83898600 & -7.84173300 & 0.44480000\end{array}$ $\begin{array}{lll}3.87558600 & -0.20724700 & 1.79174600\end{array}$ $\begin{array}{lll}1.72655000 & -1.37226100 & 1.74484400\end{array}$ $9.99767600 \quad-2.49550100-0.15443100$ $\begin{array}{lll}10.01223600 & 2.41965200 & 0.22686500\end{array}$ $\begin{array}{lll}11.26105100 & -3.15083100 & 0.05283600\end{array}$ $\begin{array}{llll}11.22402100 & -4.05439700 & -0.55639900\end{array}$ $12.07989400-2.50055500-0.26638800$ $11.39626400-3.40144700 \quad 1.10902600$ $\begin{array}{lll}11.27722700 & 3.07294900 & 0.02291800\end{array}$ $\begin{array}{lll}11.25717600 & 3.95419800 & 0.66481400\end{array}$ $\begin{array}{lll}12.09549400 & 2.40544100 & 0.30582200\end{array}$ $\begin{array}{lll}11.39806200 & 3.36035800 & -1.02567000\end{array}$ $\begin{array}{llll}4.38782300 & -4.96047600 & -1.96379200\end{array}$ $\begin{array}{llll}1.96502500 & -6.39950700 & -1.98753700\end{array}$ $\begin{array}{lll}4.38942100 & 4.91335200 & 2.01542200\end{array}$ $\begin{array}{lll}2.02491500 & 6.40480300 & 1.95123200\end{array}$ $2.72029800 \quad-7.49379100-1.45104800$ $2.42008200-7.69832500-0.41517700$ $\begin{array}{llll}2.48517100 & -8.35876100 & -2.07564600\end{array}$ $3.79488600 \quad-7.28721800-1.48696900$ $\begin{array}{llll}4.26283600 & -4.96222300 & -3.39293700\end{array}$ $\begin{array}{lll}4.03849300 & -3.95309500 & -3.76129800\end{array}$ $\begin{array}{lll}5.23273600 & -5.28326600 & -3.77975900\end{array}$ $3.48045100-5.65345000-3.72143800$ $\begin{array}{lll}5.08054100 & 6.02174100 & 1.42457500\end{array}$ $\begin{array}{lll}5.91936000 & 6.24663800 & 2.08761800\end{array}$ $\begin{array}{llll}5.46187500 & 5.75409000 & 0.43017600\end{array}$ $\begin{array}{llll}4.42324600 & 6.89423800 & 1.34549900\end{array}$ $\begin{array}{lll}2.00613800 & 6.29962700 & 3.38202600\end{array}$ $\begin{array}{lll}2.94019400 & 5.86827100 & 3.75558700\end{array}$ $\begin{array}{lll}1.88861200 & 7.31837800 & 3.75884100\end{array}$ $\begin{array}{lll}1.15710100 & 5.68763800 & 3.71250300\end{array}$ $\begin{array}{lll}-1.32800400 & -7.92469000 & 0.42636900\end{array}$ $\begin{array}{lll}-0.51638300 & -7.30262800 & 0.80488700\end{array}$ $\begin{array}{lll}-1.43927700 & -8.75382300 & 1.12939700\end{array}$ $\begin{array}{lll}-6.24962200 & -5.15157200 & 1.56222000\end{array}$ $\begin{array}{lll}-5.97973200 & -4.23616600 & 2.09229900\end{array}$ $\begin{array}{lll}-6.63149200 & -5.86160100 & 2.30078300\end{array}$ $\begin{array}{lll}-6.21692800 & 5.20099000 & -1.57604900\end{array}$ $\begin{array}{llll}-5.95457700 & 4.28384600 & -2.10693300\end{array}$ $\begin{array}{lll}-6.59545200 & 5.91374700 & -2.31369800\end{array}$ $\begin{array}{llll}-1.27394100 & 7.94064500 & -0.45734800\end{array}$ $\begin{array}{lll}-0.46512900 & 7.31307900 & -0.83201900\end{array}$ $\begin{array}{lll}-1.38170300 & 8.76548800 & -1.16604700\end{array}$ $\begin{array}{lll}-7.48599200 & -1.38290500 & 0.04730600\end{array}$ $\begin{array}{lll}-7.47643100 & 1.44095200 & -0.06361800\end{array}$ $\begin{array}{llll}-8.27344700 & -1.29839700 & -1.14854200\end{array}$ $\begin{array}{lll}-9.12889900 & -1.96005100 & -0.99338300\end{array}$ $\begin{array}{lll}-8.61900000 & -0.27483700 & -1.32472300\end{array}$ $\begin{array}{lll}-7.69580700 & -1.64383100 & -2.01517800\end{array}$ 


\section{1-Benz TS (no D3)}

C

$\begin{array}{lll}-4.10116400 & 0.83089700 & 0.27151700\end{array}$ $\begin{array}{llll}-5.32713000 & 1.54737600 & 0.16192700\end{array}$

$\begin{array}{lll}-8.26597600 & 1.36097700 & 1.13109200\end{array}$

$\begin{array}{lll}-9.11598900 & 2.02957600 & 0.97579100\end{array}$

$\begin{array}{lll}-8.61983000 & 0.33996600 & 1.30555300\end{array}$

$\begin{array}{lll}-7.68673400 & 1.70066600 & 1.99894800\end{array}$

$\begin{array}{llll}7.55999316 & -3.84097107 & -0.10508953\end{array}$

$\begin{array}{llll}6.78164129 & -4.57404078 & -0.06413622\end{array}$

$\begin{array}{llll}7.54335443 & -3.35598254 & -1.05871906\end{array}$

$\begin{array}{llll}8.92329836 & -4.52788730 & 0.09775281\end{array}$

$\begin{array}{llll}9.54735556 & -4.33950784 & -0.75075517\end{array}$

$\begin{array}{llll}9.39116701 & -4.13901034 & 0.97796508\end{array}$

$\begin{array}{lll}8.77860364 & -5.58241654 & 0.20698437\end{array}$

$\begin{array}{lll}7.58117280 & 3.78010176 & 0.16143239\end{array}$

$\begin{array}{llll}6.84308183 & 4.55153314 & 0.09061338\end{array}$

$\begin{array}{lll}7.50424242 & 3.29925348 & 1.11420027\end{array}$

$\begin{array}{llll}8.98420197 & 4.39651969 & 0.00932028\end{array}$

$\begin{array}{llll}9.57546037 & 4.15356163 & 0.86739073\end{array}$

$\begin{array}{llll}9.45265135 & 4.00455019 & -0.86920979\end{array}$

$\begin{array}{llll}8.89932571 & 5.45973750 & -0.07590832\end{array}$

$\begin{array}{lll}-0.86861208 & -8.52680710 & -0.91453147\end{array}$

$-1.22215450 \quad-7.91663918-1.71926923$

$\begin{array}{llll}0.20034340 & -8.56657889 & -0.94007355\end{array}$

$\begin{array}{llll}-1.43888029 & -9.94986795 & -1.06044915\end{array}$

$-1.74675775-10.31080832-0.10136963$

$\begin{array}{llll}-2.28018871 & -9.93093898 & -1.72131362\end{array}$

$-0.68591952-10.59660623-1.46004854$

$\begin{array}{lll}-7.41106113 & -4.80419086 & 0.61249083\end{array}$

$\begin{array}{lll}-7.88354034 & -3.90240395 & 0.94179885\end{array}$

$\begin{array}{llll}-7.03234735 & -4.66697563 & -0.37879513\end{array}$

$\begin{array}{lll}-8.43754768 & -5.95219618 & 0.61551537\end{array}$

$\begin{array}{lll}-8.19908138 & -6.64592521 & -0.16344153\end{array}$

$\begin{array}{lll}-8.40932152 & -6.45339765 & 1.56044942\end{array}$

$\begin{array}{lll}-9.41744807 & -5.55490536 & 0.45163968\end{array}$

$\begin{array}{lll}-3.85401953 & -9.37502578 & 0.30201610\end{array}$

$\begin{array}{lll}-3.72097418 & -9.63878876 & -0.72639433\end{array}$

$\begin{array}{lll}-3.06019334 & -9.79519097 & 0.88356679\end{array}$

$\begin{array}{lll}-4.79133646 & -9.75643696 & 0.64966892\end{array}$

$\begin{array}{lll}-6.36043089 & -7.93414429 & 0.78396682\end{array}$

$\begin{array}{llll}-6.92129481 & -7.59218429 & 1.62859396\end{array}$

$\begin{array}{lll}-6.92828070 & -7.77590446 & -0.10900953\end{array}$

$\begin{array}{lll}-6.15139975 & -8.97779852 & 0.89347401\end{array}$

$\begin{array}{llll}-7.37878086 & 4.86151309 & -0.62397055\end{array}$

$\begin{array}{lll}-7.85780111 & 3.96274852 & -0.95208920\end{array}$

$\begin{array}{lll}-6.99905791 & 4.72210682 & 0.36662340\end{array}$

$\begin{array}{lll}-8.39771937 & 6.01622621 & -0.62536165\end{array}$

$\begin{array}{lll}-8.15100718 & 6.71053722 & 0.15050259\end{array}$

$\begin{array}{llll}-8.37070063 & 6.51460107 & -1.57182480\end{array}$

$\begin{array}{lll}-9.37941381 & 5.62584064 & -0.45572928\end{array}$

$\begin{array}{lll}-0.80812609 & 8.55020751 & 0.87796096\end{array}$

$\begin{array}{llll}-1.21774139 & 7.98611677 & 1.68969794\end{array}$

$\begin{array}{llll}0.26034715 & 8.52379272 & 0.92862854\end{array}$

$\begin{array}{lll}-1.29057421 & 10.00965630 & 0.97205155\end{array}$

$\begin{array}{lll}-1.60864010 & 10.34312269 & 0.00637341\end{array}$

$\begin{array}{lll}-2.10934734 & 10.07111447 & 1.65815172\end{array}$

$\begin{array}{lll}-0.48894247 & 10.62876394 & 1.31700413\end{array}$

$\begin{array}{lll}-6.30693981 & 7.98434365 & -0.79781967\end{array}$

$\begin{array}{lll}-6.84365788 & 7.69015812 & -1.67548053\end{array}$

$\begin{array}{lll}-6.90114636 & 7.78112269 & 0.06850580\end{array}$

$\begin{array}{lll}-6.09211183 & 9.03147749 & -0.84535556\end{array}$

$\begin{array}{lll}-3.78965336 & 9.40806916 & -0.32317954\end{array}$

$\begin{array}{lll}-3.70977238 & 9.67040801 & 0.71108221\end{array}$

$\begin{array}{lll}-2.96026415 & 9.81852303 & -0.86033980\end{array}$

$\begin{array}{lll}-4.70193442 & 9.80068344 & -0.72129672\end{array}$

C
C
C

C

C

C

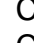

8

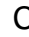

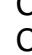

C

C

H

$\mathrm{H}$

$\mathrm{H}$

$\mathrm{H}$

$\mathrm{H}$

$\mathrm{H}$

C

C

C

C

C

C

C

C

C

C

O

C

$\mathrm{H}$

$\mathrm{H}$

C

$\mathrm{H}$

$\mathrm{H}$

C

C

C

C

C

C

C

C

C

$\mathrm{C}$

$\mathrm{C}$

$\mathrm{C}$

C

C $\begin{array}{lll}-4.11599000 & -0.59633000 & 0.56773900\end{array}$

$\begin{array}{lll}-2.95487500 & -1.38238900 & 0.61862000\end{array}$

$\begin{array}{lll}-2.98713000 & -2.76870400 & 0.78151400\end{array}$

$\begin{array}{lll}-4.23410200 & -3.43195100 & 0.92498400\end{array}$

$\begin{array}{lll}-5.39493100 & -2.66013300 & 0.86313600\end{array}$

$\begin{array}{lll}-1.78957700 & -3.57625400 & 0.55210400\end{array}$

$\begin{array}{lll}-1.97522200 & -4.88105800 & -0.02878600\end{array}$

$\begin{array}{lll}-3.22753100 & -5.62667000 & 0.31089300\end{array}$

$\begin{array}{lll}-4.24567400 & -4.90674300 & 1.02139000\end{array}$

$\begin{array}{lll}-0.51162900 & -3.03915700 & 0.68604900\end{array}$

$\begin{array}{lll}0.60784500 & -3.60466100 & 0.05448200\end{array}$

$\begin{array}{lll}-0.89920800 & -5.33209700 & -0.80538800\end{array}$

$\begin{array}{lll}1.94714100 & -3.05533500 & 0.14575000\end{array}$

$\begin{array}{lll}-5.36741100 & -1.26388700 & 0.69853100\end{array}$

$0.35594200-4.69146100-0.82646300$

$\begin{array}{lll}2.89107200 & -3.37954400 & -0.86905900\end{array}$

$\begin{array}{lll}4.11847600 & -2.68012800 & -0.93070200\end{array}$

$\begin{array}{lll}2.37306500 & -2.20394700 & 1.18921200\end{array}$

$\begin{array}{lll}-6.57114300 & 0.84968500 & 0.38676000\end{array}$

$2.57468500-4.41691000-1.82067800$

$3.61893200 \quad-1.60771600 \quad 1.16326900$

$\begin{array}{lll}-6.59133100 & -0.50259200 & 0.60752500\end{array}$

$\begin{array}{lll}1.39043100 & -5.10747000 & -1.74123400\end{array}$

$\begin{array}{llll}4.47859300 & -1.75557100 & 0.04269200\end{array}$

$\begin{array}{lll}-1.99711700 & -0.91712900 & 0.42218100\end{array}$

$\begin{array}{lll}-6.36456700 & -3.13359500 & 0.89204300\end{array}$

$\begin{array}{lll}-0.39619700 & -2.10323400 & 1.22331000\end{array}$

$\begin{array}{lll}-0.99256400 & -6.16740900 & -1.47303200\end{array}$

$\begin{array}{lll}4.76206900 & -2.86744500 & -1.77990500\end{array}$

$\begin{array}{lll}1.71393000 & -2.02668200 & 2.03409200\end{array}$

$\begin{array}{lll}3.92125400 & -0.95277000 & 1.97487500\end{array}$

$\begin{array}{lll}-5.21477900 & -5.59384000 & 1.79845100\end{array}$

$\begin{array}{lll}-5.27392100 & -6.99636100 & 1.73024900\end{array}$

$\begin{array}{lll}-4.44816200 & -7.69366500 & 0.82431800\end{array}$

$\begin{array}{lll}-3.39860800 & -7.03717400 & 0.14800100\end{array}$

$\begin{array}{lll}5.73196500 & -0.92205300 & 0.02379400\end{array}$

$\begin{array}{lll}6.95894800 & -1.61355400 & 0.17008500\end{array}$

$\begin{array}{lll}8.08899300 & -0.88988600 & 0.57184100\end{array}$

$\begin{array}{lll}7.96784000 & 0.46310700 & 0.90152500\end{array}$

$\begin{array}{lll}6.79865500 & 1.20014700 & 0.64255600\end{array}$

$\begin{array}{lll}5.66253800 & 0.52117500 & 0.07974000\end{array}$

$\begin{array}{llll}9.19391700 & 1.10386100 & 1.50805300\end{array}$

$\begin{array}{llll}9.40991800 & -1.55633600 & 0.85040700\end{array}$

$\begin{array}{llll}9.04449700 & 1.31308700 & 2.83118500\end{array}$

$\begin{array}{lll}9.97927000 & -2.06646000 & -0.25902900\end{array}$

$\begin{array}{lll}6.84775700 & 2.66065200 & 1.10196800\end{array}$

$\begin{array}{lll}7.84440600 & 2.86358900 & 1.49641000\end{array}$

$\begin{array}{lll}6.75525900 & 3.36606800 & 0.27430300\end{array}$

$\begin{array}{lll}7.07420100 & -3.11828900 & -0.02560600\end{array}$

$\begin{array}{lll}6.14005500 & -3.60243400 & 0.26962900\end{array}$

$\begin{array}{lll}7.84345300 & -3.51640600 & 0.64711500\end{array}$

$\begin{array}{lll}-2.92329800 & 1.55749900 & 0.07429700\end{array}$

$\begin{array}{lll}-2.90815000 & 2.89860800 & -0.30721200\end{array}$

$\begin{array}{llll}-4.13237600 & 3.61196300 & -0.46014600\end{array}$

$\begin{array}{llll}-5.30996100 & 2.91574500 & -0.16337600\end{array}$

$\begin{array}{lll}-1.64809000 & 3.59965500 & -0.52730900\end{array}$

$\begin{array}{llll}-1.62437400 & 5.02843200 & -0.50457900\end{array}$

$\begin{array}{lll}-2.90350000 & 5.77476800 & -0.44292800\end{array}$

$\begin{array}{llll}-4.09985100 & 5.06130500 & -0.76927200\end{array}$

$\begin{array}{lll}-0.47292600 & 2.88913000 & -0.74295800\end{array}$

$\begin{array}{lll}0.77687800 & 3.50290200 & -0.85881600\end{array}$

$\begin{array}{lll}-0.38171900 & 5.64633800 & -0.70158000\end{array}$

$\begin{array}{lll}2.00569700 & 2.74374600 & -0.91614300\end{array}$

$\begin{array}{llll}0.81932400 & 4.92394700 & -0.82633300\end{array}$

$\begin{array}{llll}3.25426100 & 3.40819600 & -0.84825400\end{array}$

$\begin{array}{llll}2.05501300 & 1.34132800 & -0.99571600\end{array}$

$\begin{array}{llll}4.46118200 & 1.27438100 & -0.46827500\end{array}$ 


\section{1-Se GS (no D3)}

C
C
C
C
C
C $\begin{array}{llll}7.42748400 & -3.54167600 & -1.47008700\end{array}$ $6.75924300-3.03751400 \quad-2.17833000$ $8.43542200 \quad-3.18707600 \quad-1.70380500$ $\begin{array}{lll}7.33350600 & -5.05742200 & -1.66905400\end{array}$ $\begin{array}{lll}7.96647300 & -5.59357800 & -0.95059700\end{array}$ $\begin{array}{llll}6.30314300 & -5.40913100 & -1.53992500\end{array}$ $\begin{array}{lll}7.65878100 & -5.34403600 & -2.67595900\end{array}$ $\begin{array}{lll}5.85275500 & 3.01015900 & 2.22588800\end{array}$ $\begin{array}{lll}4.83191300 & 2.74999500 & 1.92939800\end{array}$ $\begin{array}{lll}6.09599200 & 2.38920600 & 3.09753000\end{array}$ $\begin{array}{lll}5.91375300 & 4.49386400 & 2.60230000\end{array}$ $\begin{array}{llll}6.92410400 & 4.78500000 & 2.91691400\end{array}$ $\begin{array}{llll}5.63282100 & 5.12447700 & 1.75015300\end{array}$ $\begin{array}{lll}5.23079800 & 4.72003300 & 3.42923400\end{array}$ $\begin{array}{lll}-6.20187400 & -7.79468200 & 2.62864400\end{array}$ $\begin{array}{lll}-6.53375600 & -7.22318200 & 3.49543100\end{array}$ $\begin{array}{lll}-7.10487700 & -8.13051600 & 2.10068800\end{array}$ $\begin{array}{lll}-5.70427700 & -8.69232500 & 3.00893300\end{array}$ $\begin{array}{lll}-4.67566300 & -9.18859900 & 0.65828300\end{array}$ $\begin{array}{lll}-4.01839300 & -9.78380000 & 1.30880300\end{array}$ $\begin{array}{lll}-5.70245400 & -9.46352600 & 0.90585900\end{array}$ $-4.50523000 \quad-9.52247600 \quad-0.36512000$ $\begin{array}{lll}-2.66120300 & -7.75243500 & -2.22774900\end{array}$ $\begin{array}{lll}-2.65765600 & -6.69322300 & -2.50964800\end{array}$ $\begin{array}{llll}-1.79680900 & -8.20725300 & -2.73101900\end{array}$ $\begin{array}{lll}-3.94723000 & -8.38963100 & -2.76524000\end{array}$ $-3.96143200 \quad-9.47402200-2.60066900$ $\begin{array}{lll}-4.83532900 & -7.96200800 & -2.28655400\end{array}$ $\begin{array}{llll}-4.03907300 & -8.22118900 & -3.84436800\end{array}$ $\begin{array}{lll}-7.62098000 & -4.82271100 & 2.43052300\end{array}$ $\begin{array}{lll}-7.75355500 & -4.49416700 & 1.39208200\end{array}$ $\begin{array}{lll}-8.03704600 & -5.83601500 & 2.47166300\end{array}$ $\begin{array}{lll}-8.41558800 & -3.90000400 & 3.36023600\end{array}$ $\begin{array}{lll}-8.27944000 & -4.18117400 & 4.41217700\end{array}$ $\begin{array}{lll}-8.09568900 & -2.85844400 & 3.24318400\end{array}$ $\begin{array}{lll}-9.48874500 & -3.94491300 & 3.14088800\end{array}$ $\begin{array}{lll}-6.23427100 & 7.99434500 & -2.04106900\end{array}$ $\begin{array}{lll}-6.80354200 & 7.39498800 & -2.75106900\end{array}$ $\begin{array}{lll}-6.95789500 & 8.44192800 & -1.34676800\end{array}$ $\begin{array}{lll}-5.78605100 & 8.81632400 & -2.60907700\end{array}$ $\begin{array}{llll}-4.26746800 & 9.36206700 & -0.38505500\end{array}$ $\begin{array}{lll}-3.41131500 & 9.91994100 & -0.78279500\end{array}$ $\begin{array}{lll}-5.16403300 & 9.77985900 & -0.84071900\end{array}$ $\begin{array}{lll}-4.32711400 & 9.58979700 & 0.68657600\end{array}$ $\begin{array}{lll}-7.72679800 & 5.13361400 & -1.35014600\end{array}$ $\begin{array}{lll}-7.64330000 & 4.84070200 & -0.29600200\end{array}$ $\begin{array}{llll}-8.07338200 & 6.17331300 & -1.33669000\end{array}$ $\begin{array}{lll}-8.76881100 & 4.24913500 & -2.04260200\end{array}$ $\begin{array}{lll}-8.85464400 & 4.49494100 & -3.10865900\end{array}$ $\begin{array}{lll}-8.50028000 & 3.19007800 & -1.95838400\end{array}$ $\begin{array}{lll}-9.75999700 & 4.37808800 & -1.59205200\end{array}$ $\begin{array}{lll}-1.54291300 & 7.27003500 & 1.97323700\end{array}$ $\begin{array}{lll}-1.07298200 & 6.29943300 & 1.78923200\end{array}$ $\begin{array}{lll}-0.77090500 & 7.90447100 & 2.43094300\end{array}$ $\begin{array}{lll}-2.70329900 & 7.09897400 & 2.95994000\end{array}$ $\begin{array}{lll}-3.16842600 & 8.06388000 & 3.19973300\end{array}$ $\begin{array}{lll}-3.48122300 & 6.44626300 & 2.54957700\end{array}$

$\begin{array}{lll}-2.35636400 & 6.65512500 & 3.90009100\end{array}$

$\begin{array}{llll}\text { C } & -4.09460800 & 0.84468500 & 0.24528300 \\ \text { C } & -5.31780200 & 1.56311800 & 0.12665400 \\ \text { C } & -4.11413000 & -0.58013600 & 0.54303800 \\ \text { C } & -2.95575900 & -1.36713800 & 0.60772300 \\ \text { C } & -2.99269600 & -2.75245500 & 0.77428400 \\ \text { C } & -4.24367300 & -3.41536000 & 0.89057000\end{array}$




\begin{tabular}{|c|c|c|c|}
\hline C & -5.40093100 & -2.64094300 & 0.80721700 \\
\hline C & -1.79114500 & -3.55739200 & 0.56419200 \\
\hline 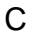 & -1.96986900 & -4.86756700 & -0.00477400 \\
\hline C & -3.21657800 & -5.61748500 & 0.33635100 \\
\hline C & -4.26042200 & -4.88867800 & 0.99677700 \\
\hline C & -0.51600600 & -3.01181400 & 0.69317900 \\
\hline C & 0.60591600 & -3.57803600 & 0.06741400 \\
\hline C & -0.89560200 & -5.31533100 & -0.78247600 \\
\hline C & 1.94395300 & -3.02707300 & 0.15562400 \\
\hline C & -5.36747800 & -1.24450400 & 0.66091700 \\
\hline C & 0.35691600 & -4.67361000 & -0.80382700 \\
\hline 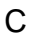 & 2.89898400 & -3.38743400 & -0.83658200 \\
\hline$\checkmark$ & 4.12862500 & -2.69706200 & -0.90735500 \\
\hline C & 2.36096400 & -2.14466900 & 1.17704900 \\
\hline C & -6.56450300 & 0.86729000 & 0.33145000 \\
\hline$\checkmark$ & 2.59487800 & -4.45103300 & -1.76007100 \\
\hline$\checkmark$ & 3.61129700 & -1.55910600 & 1.14860600 \\
\hline C & -6.58796300 & -0.48089700 & 0.56957200 \\
\hline C & 1.39498700 & -5.11279300 & -1.70020400 \\
\hline $\mathrm{C}$ & 4.48028500 & -1.74773300 & 0.04216800 \\
\hline $\mathrm{H}$ & -1.99600100 & -0.90264700 & 0.42036300 \\
\hline $\mathrm{H}$ & -6.37219600 & -3.10883300 & 0.80501700 \\
\hline $\mathrm{H}$ & -0.40539800 & -2.06972100 & 1.22022800 \\
\hline $\mathrm{H}$ & -0.98744500 & -6.15076700 & -1.44896300 \\
\hline $\mathrm{H}$ & 4.78099700 & -2.91070000 & -1.74227000 \\
\hline $\mathrm{H}$ & 1.69177500 & -1.93525800 & 2.00621300 \\
\hline $\mathrm{H}$ & 3.90932800 & -0.87409400 & 1.93646100 \\
\hline C & -5.25869600 & -5.56652400 & 1.74447200 \\
\hline C & -5.31363500 & -6.96870300 & 1.69555000 \\
\hline C & -4.43155400 & -7.68412600 & 0.85972700 \\
\hline C & -3.35531100 & -7.03615900 & 0.22078000 \\
\hline C & -2.91513800 & 1.56639000 & 0.04785900 \\
\hline C & -2.89706900 & 2.90515700 & -0.33874900 \\
\hline$C$ & -4.11814800 & 3.62926500 & -0.47428000 \\
\hline C & -5.29674400 & 2.93398100 & -0.18118500 \\
\hline C & -1.63435400 & 3.59045600 & -0.58084400 \\
\hline C & -1.59916800 & 5.01742700 & -0.56182500 \\
\hline C & -2.86438600 & 5.77552400 & -0.45873600 \\
\hline C & -4.07809700 & 5.08271400 & -0.75969200 \\
\hline C & -0.46884400 & 2.86897100 & -0.81476900 \\
\hline C & 0.78368200 & 3.47460400 & -0.94104400 \\
\hline C & -0.35935600 & 5.62734800 & -0.78517200 \\
\hline C & 2.01007200 & 2.71278500 & -1.00007500 \\
\hline C & 0.83339700 & 4.89588600 & -0.91310900 \\
\hline C & 3.25911400 & 3.37700300 & -0.93304400 \\
\hline C & 2.05866900 & 1.30897500 & -1.07309300 \\
\hline C & 4.46606900 & 1.24734800 & -0.55033400 \\
\hline C & 4.44384500 & 2.63538400 & -0.74041200 \\
\hline C & 3.22594700 & 0.61202800 & -0.86380000 \\
\hline C & 3.28180000 & 4.82229500 & -0.95663400 \\
\hline C & 2.11802100 & 5.54789700 & -0.95957600 \\
\hline $\mathrm{H}$ & -1.96991000 & 1.07023300 & 0.22104900 \\
\hline $\mathrm{H}$ & -6.24551900 & 3.44124900 & -0.16250400 \\
\hline $\mathrm{H}$ & -0.53104000 & 1.79120400 & -0.87859300 \\
\hline $\mathrm{H}$ & -0.27903300 & 6.69946400 & -0.86095600 \\
\hline $\mathrm{H}$ & 5.33988000 & 3.22035600 & -0.68798600 \\
\hline C & -5.18617900 & 5.82602700 & -1.25861400 \\
\hline C & -5.13980200 & 7.23060600 & -1.22022200 \\
\hline C & -2.93032700 & 7.16842800 & -0.16824900 \\
\hline C & -4.07752100 & 7.88437100 & -0.54902400 \\
\hline $\mathrm{H}$ & 3.15977900 & -0.45189600 & -0.96964600 \\
\hline L & 1.15854800 & 0.73718300 & -1.27329400 \\
\hline ه & 4.51143300 & 5.44085200 & -0.87192500 \\
\hline $\mathrm{O}$ & 2.14538800 & 6.92885400 & -0.94803400 \\
\hline C & 4.89758000 & 6.19619600 & -2.02875000 \\
\hline 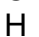 & 4.21100900 & 7.03078800 & -2.19857300 \\
\hline I & 5.90170900 & 6.57250600 & -1.82022500 \\
\hline
\end{tabular}

\begin{tabular}{|c|c|c|c|}
\hline $\mathrm{H}$ & 4.92514200 & 5.55268900 & -2.91715400 \\
\hline C & 2.43149400 & 7.48249900 & 0.34378100 \\
\hline $\mathrm{H}$ & 3.41988200 & 7.16543200 & 0.69461500 \\
\hline $\mathrm{H}$ & 2.41142500 & 8.56837200 & 0.22283300 \\
\hline $\mathrm{H}$ & 1.66656800 & 7.18121600 & 1.07161200 \\
\hline C & -6.37244800 & 5.13782800 & -1.92256900 \\
\hline $\mathrm{H}$ & -6.12519000 & 4.09756500 & -2.14329300 \\
\hline $\mathrm{H}$ & -6.54272300 & 5.59697100 & -2.90508200 \\
\hline C & -1.85433700 & 7.88862200 & 0.64890100 \\
\hline $\mathrm{H}$ & -0.98319700 & 8.15932900 & 0.04106500 \\
\hline $\mathrm{H}$ & -2.26053800 & 8.84569100 & 0.98020800 \\
\hline $\mathrm{O}$ & -7.73005900 & 1.59435300 & 0.20536100 \\
\hline $\mathrm{O}$ & -7.77673700 & -1.15526100 & 0.76046600 \\
\hline C & -8.46500100 & 1.77389200 & 1.42283500 \\
\hline $\mathrm{H}$ & -8.81116000 & 0.81350900 & 1.81832300 \\
\hline $\mathrm{H}$ & -7.84736900 & 2.28118900 & 2.17477100 \\
\hline $\mathrm{H}$ & -9.31973800 & 2.40452900 & 1.16752500 \\
\hline C & -8.58434200 & -1.29580800 & -0.41597800 \\
\hline $\mathrm{H}$ & -8.03335000 & -1.83156400 & -1.19935200 \\
\hline $\mathrm{H}$ & -9.45473200 & -1.88360400 & -0.11533300 \\
\hline $\mathrm{H}$ & -8.90291000 & -0.31868500 & -0.79311300 \\
\hline C & -6.22047500 & -4.82226400 & 2.66248800 \\
\hline $\mathrm{H}$ & -5.86532000 & -3.80471500 & 2.83303700 \\
\hline $\mathrm{H}$ & -6.20516700 & -5.29410500 & 3.65345700 \\
\hline C & -2.35700900 & -7.89077600 & -0.54189300 \\
\hline $\mathrm{H}$ & -1.34303400 & -7.58237900 & -0.27598500 \\
\hline $\mathrm{H}$ & -2.41906200 & -8.93111200 & -0.21714100 \\
\hline $\mathrm{O}$ & 1.10944500 & -6.16909300 & -2.53875800 \\
\hline $\mathrm{O}$ & 3.58977600 & -4.82557800 & -2.63762400 \\
\hline C & 1.87286700 & -7.35579800 & -2.28234900 \\
\hline $\mathrm{H}$ & 1.50745700 & -8.10759400 & -2.98575100 \\
\hline $\mathrm{H}$ & 2.94162900 & -7.18076100 & -2.44291500 \\
\hline $\mathrm{H}$ & 1.70832300 & -7.70563900 & -1.25517100 \\
\hline C & 3.28902700 & -4.61416500 & -4.02431100 \\
\hline $\mathrm{H}$ & 4.18518900 & -4.90989300 & -4.57432900 \\
\hline $\mathrm{H}$ & 2.43577700 & -5.22216300 & -4.34071000 \\
\hline $\mathrm{H}$ & 3.07520100 & -3.55476400 & -4.21380800 \\
\hline C & -6.30484300 & -7.75316600 & 2.53550000 \\
\hline $\mathrm{H}$ & -6.70740900 & -7.17001100 & 3.36315400 \\
\hline $\mathrm{H}$ & -7.16069300 & -8.09848200 & 1.93971800 \\
\hline $\mathrm{H}$ & -5.84025100 & -8.64426000 & 2.96938300 \\
\hline C & -4.64242900 & -9.18538300 & 0.74575400 \\
\hline $\mathrm{H}$ & -4.17273100 & -9.72576400 & 1.58016900 \\
\hline $\mathrm{H}$ & -5.70644300 & -9.43592700 & 0.76453700 \\
\hline $\mathrm{H}$ & -4.24470500 & -9.60376100 & -0.17557900 \\
\hline C & -2.53644300 & -7.84393600 & -2.08063300 \\
\hline $\mathrm{H}$ & -2.58883000 & -6.79960500 & -2.40905000 \\
\hline $\mathrm{H}$ & -1.64179800 & -8.27259100 & -2.55322100 \\
\hline C & -3.78347400 & -8.57013500 & -2.59512600 \\
\hline $\mathrm{H}$ & -3.73951900 & -9.64601800 & -2.38661000 \\
\hline $\mathrm{H}$ & -4.69200300 & -8.17152100 & -2.12970300 \\
\hline $\mathrm{H}$ & -3.88248200 & -8.44883500 & -3.67975100 \\
\hline C & -7.68593100 & -4.77231200 & 2.17503600 \\
\hline $\mathrm{H}$ & -7.71453200 & -4.51869300 & 1.10779000 \\
\hline $\mathrm{H}$ & -8.13312000 & -5.77054700 & 2.24773800 \\
\hline C & -8.52409700 & -3.76168300 & 2.96302600 \\
\hline $\mathrm{H}$ & -8.47564600 & -3.96337900 & 4.04060100 \\
\hline $\mathrm{H}$ & -8.16513800 & -2.74188400 & 2.78648900 \\
\hline $\mathrm{H}$ & -9.57823900 & -3.80040600 & 2.66367200 \\
\hline C & -6.23444100 & 8.07094900 & -1.85463600 \\
\hline $\mathrm{H}$ & -6.85392700 & 7.49986000 & -2.54550400 \\
\hline $\mathrm{H}$ & -6.90813500 & 8.50468300 & -1.10400700 \\
\hline $\mathrm{H}$ & -5.81001500 & 8.90319700 & -2.42579600 \\
\hline C & -4.16924100 & 9.37448000 & -0.26312700 \\
\hline $\mathrm{H}$ & -3.30539500 & 9.92381200 & -0.65532800 \\
\hline $\mathrm{H}$ & -5.06129200 & 9.82941100 & -0.69113500 \\
\hline $\mathrm{H}$ & -4.20814800 & 9.56610000 & 0.81667600 \\
\hline
\end{tabular}




$\begin{array}{lccc}\mathrm{C} & -7.69706800 & 5.19318900 & -1.12979400 \\ \mathrm{H} & -7.51632800 & 4.92244600 & -0.08161300 \\ \mathrm{H} & -8.06482100 & 6.22539100 & -1.10269500 \\ \mathrm{C} & -8.76489400 & 4.26851500 & -1.72119600 \\ \mathrm{H} & -8.94064600 & 4.49464500 & -2.78054500 \\ \mathrm{H} & -8.45592900 & 3.22062100 & -1.63988200 \\ \mathrm{H} & -9.72070800 & 4.37779300 & -1.19481400 \\ \mathrm{C} & -1.39377400 & 7.15323700 & 1.92603900 \\ \mathrm{H} & -0.88036100 & 6.22101600 & 1.67486300 \\ \mathrm{H} & -0.65146000 & 7.79080800 & 2.42631800 \\ \mathrm{C} & -2.54784800 & 6.85748300 & 2.88983900 \\ \mathrm{H} & -3.07115300 & 7.77893300 & 3.17604300 \\ \mathrm{H} & -3.28122200 & 6.18768400 & 2.42841800 \\ \mathrm{H} & -2.18419300 & 6.37951000 & 3.80637800 \\ \mathrm{C} & 7.36914307 & -1.40360048 & 0.36408665 \\ \mathrm{C} & 6.04167030 & -0.99901296 & 0.16653147 \\ \mathrm{C} & 6.02287016 & 0.43471528 & 0.22062285 \\ \mathrm{C} & 7.33957566 & 0.85788005 & 0.44952668 \\ \mathrm{Se} & 8.15425806 & -0.26727699 & 0.53573918 \\ \mathrm{C} & 7.97959952 & -2.81682164 & 0.40590626 \\ \mathrm{H} & 7.20876067 & -3.54298294 & 0.25294472 \\ \mathrm{H} & 8.43845224 & -2.97908022 & 1.35881042 \\ \mathrm{C} & 9.03908250 & -2.95138179 & -0.70359334 \\ \mathrm{H} & 9.95436208 & -2.50206694 & -0.37912538 \\ \mathrm{H} & 8.69372089 & -2.45839426 & -1.58823375 \\ \mathrm{C} & 9.27830924 & -4.44215275 & -1.00686303 \\ \mathrm{H} & 10.07885549 & -4.53905807 & -1.71016599 \\ \mathrm{H} & 9.53422494 & -4.95351107 & -0.10247383 \\ \mathrm{H} & 8.38806315 & -4.86968409 & -1.41866255 \\ \mathrm{C} & 7.91319853 & 2.27925466 & 0.59869445 \\ \mathrm{H} & 8.63022733 & 2.45753918 & -0.17524633 \\ \mathrm{H} & 8.38754088 & 2.37409641 & 1.55310756 \\ \mathrm{C} & 6.77213748 & 3.30753103 & 0.48811702 \\ \mathrm{H} & 6.30263287 & 3.21774785 & -0.46917312 \\ \mathrm{H} & 6.05134087 & 3.12456971 & 1.25745271 \\ \mathrm{C} & 7.34422068 & 4.72835513 & 0.64805356 \\ \mathrm{H} & 6.55139097 & 5.44280149 & 0.57132391 \\ \mathrm{H} & 7.81381330 & 4.81810648 & 1.60530351 \\ \mathrm{H} & 8.06494401 & 4.91135296 & -0.12134210 \\ & & & \\ & & & \end{array}$

\section{1-Se TS (no D3)}

\begin{tabular}{|c|c|c|c|}
\hline C & 3.39631000 & 1.36621700 & -0.28625000 \\
\hline C & 4.48373000 & 2.27286900 & -0.12619300 \\
\hline $\mathrm{C}$ & 3.65414700 & -0.05153300 & -0.51531700 \\
\hline $\mathrm{C}$ & 2.63973300 & -1.01990200 & -0.59977300 \\
\hline $\mathrm{C}$ & 2.90570900 & -2.38849300 & -0.69512000 \\
\hline $\mathrm{C}$ & 4.25081900 & -2.84143900 & -0.73133500 \\
\hline C & 5.26478400 & -1.88850000 & -0.63402000 \\
\hline C & 1.84482000 & -3.38135300 & -0.51609900 \\
\hline C & 2.19895600 & -4.61988100 & 0.13104600 \\
\hline $\mathrm{C}$ & 3.58353700 & -5.14957000 & -0.07626500 \\
\hline $\mathrm{C}$ & 4.51364400 & -4.29517500 & -0.75652800 \\
\hline $\mathrm{C}$ & 0.51015300 & -3.08263200 & -0.77874600 \\
\hline $\mathrm{C}$ & -0.54775600 & -3.82886900 & -0.23158600 \\
\hline $\mathrm{C}$ & 1.15051500 & -5.23627900 & 0.82775200 \\
\hline $\mathrm{C}$ & -1.94788500 & -3.53783300 & -0.47154800 \\
\hline $\mathrm{C}$ & 5.00248700 & -0.51098700 & -0.53764500 \\
\hline $\mathrm{C}$ & -0.19451600 & -4.83168200 & 0.71099500 \\
\hline $\mathrm{C}$ & -2.91763100 & -4.00012700 & 0.46398800 \\
\hline $\mathrm{C}$ & -4.24538900 & -3.51147200 & 0.39045100 \\
\hline $\mathrm{C}$ & -2.40583900 & -2.80121400 & -1.58689400 \\
\hline C & 5.83467300 & 1.77741500 & -0.24633300 \\
\hline C & -2.51514100 & -4.93636800 & 1.48452000 \\
\hline C & -3.72636300 & -2.41149200 & -1.68859800 \\
\hline $\mathrm{C}$ & 6.08217900 & 0.43950700 & -0.40668400 \\
\hline $\mathrm{C}$ & -1.22411400 & -5.40351400 & 1.54356800 \\
\hline
\end{tabular}

$-4.64153400-2.65936800 \quad-0.63182700$ $\begin{array}{lll}1.60756100 & -0.71120300 & -0.48740800\end{array}$ $\begin{array}{llll}6.29701000 & -2.19885700 & -0.57864900\end{array}$ $\begin{array}{lll}0.28261200 & -2.19420000 & -1.35923800\end{array}$ $\begin{array}{lll}1.32512800 & -6.02822600 & 1.53067900\end{array}$ $\begin{array}{lll}-4.94154600 & -3.78348300 & 1.17637300\end{array}$ $\begin{array}{lll}-1.70697500 & -2.54178500 & -2.37684800\end{array}$ $-4.05363500 \quad-1.82145000-2.53969400$ $\begin{array}{llll}5.63607300 & -4.83669900 & -1.43675400\end{array}$ $\begin{array}{llll}5.92733300 & -6.20374400 & -1.29000700\end{array}$ $\begin{array}{llll}5.17538500 & -6.99131600 & -0.39371400\end{array}$ $\begin{array}{llll}3.98477600 & -6.49827500 & 0.18005200\end{array}$ $\begin{array}{llll}2.10997200 & 1.90494000 & -0.19818300\end{array}$ $\begin{array}{lll}1.85816200 & 3.23567500 & 0.13774200\end{array}$ $\begin{array}{llll}2.94089900 & 4.13750700 & 0.35103200\end{array}$ $\begin{array}{lll}4.23046100 & 3.62902500 & 0.15009400\end{array}$ $\begin{array}{lll}0.49115200 & 3.72646900 & 0.27091800\end{array}$ $\begin{array}{lll}0.23309900 & 5.13112100 & 0.25252700\end{array}$ $\begin{array}{lll}1.37662700 & 6.07297800 & 0.23108200\end{array}$ $\begin{array}{llll}2.65739200 & 5.56824700 & 0.62128100\end{array}$ $\begin{array}{lll}-0.56067200 & 2.83332300 & 0.42123300\end{array}$ $\begin{array}{lll}-1.89622700 & 3.23199300 & 0.49199400\end{array}$ $\begin{array}{lll}-1.09890900 & 5.53661300 & 0.42476200\end{array}$ $\begin{array}{lll}-2.97971000 & 2.27646200 & 0.48232900\end{array}$ $\begin{array}{lll}-2.17018800 & 4.62617000 & 0.50505400\end{array}$ $\begin{array}{lll}-4.32673100 & 2.71820600 & 0.46865000\end{array}$ $\begin{array}{lll}-2.76860600 & 0.88952200 & 0.43314900\end{array}$ $\begin{array}{lll}-5.14518900 & 0.42603600 & 0.02069000\end{array}$ $\begin{array}{lll}-5.37630300 & 1.79057700 & 0.26306500\end{array}$ $\begin{array}{lll}-3.79805800 & 0.01209900 & 0.20145900\end{array}$ $\begin{array}{lll}-4.57704900 & 4.13863500 & 0.59344100\end{array}$ $\begin{array}{lll}-3.54862700 & 5.05075800 & 0.59130200\end{array}$ $\begin{array}{llll}1.26874300 & 1.26185300 & -0.41751700\end{array}$ $\begin{array}{llll}5.08884400 & 4.27936600 & 0.18783600\end{array}$ $\begin{array}{lll}-0.33294600 & 1.77997300 & 0.48919000\end{array}$ $\begin{array}{lll}-1.34589400 & 6.58133900 & 0.52639000\end{array}$ $\begin{array}{lll}-6.37032100 & 2.20343000 & 0.26134500\end{array}$ $\begin{array}{lll}3.59859200 & 6.46757700 & 1.20362900\end{array}$ $\begin{array}{lll}3.34390500 & 7.84955600 & 1.15317800\end{array}$ $\begin{array}{lll}1.25757600 & 7.46237500 & -0.06684200\end{array}$ $\begin{array}{llll}2.25818500 & 8.33966900 & 0.38556800\end{array}$ $\begin{array}{lll}-3.54270900 & -1.02778700 & 0.18787000\end{array}$ $\begin{array}{lll}-1.77141000 & 0.47783400 & 0.53818000\end{array}$ $\begin{array}{lll}-5.89404300 & 4.55488900 & 0.64163700\end{array}$ $\begin{array}{lll}-3.79410000 & 6.40620500 & 0.68249700\end{array}$ $\begin{array}{lll}-6.30800000 & 5.05645100 & 1.91954000\end{array}$ $\begin{array}{lll}-5.74036700 & 5.95142400 & 2.19249600\end{array}$ $\begin{array}{lll}-7.36757400 & 5.30370500 & 1.82026100\end{array}$ $\begin{array}{lll}-6.18381300 & 4.29012300 & 2.69472800\end{array}$ $\begin{array}{lll}-4.38984300 & 6.98971400 & -0.48322700\end{array}$ $\begin{array}{lll}-5.37299500 & 6.55357000 & -0.68452800\end{array}$ $\begin{array}{lll}-4.49355000 & 8.05610600 & -0.26835400\end{array}$ $-3.74154300 \quad 6.85585700 \quad-1.35852600$ $\begin{array}{lll}4.80522900 & 5.95998600 & 1.98777200\end{array}$ $\begin{array}{lll}4.70484200 & 4.88775200 & 2.16681600\end{array}$ $\begin{array}{lll}4.78273100 & 6.41419900 & 2.98790400\end{array}$ $\begin{array}{llll}0.13892300 & 8.01592300 & -0.95386600\end{array}$ $\begin{array}{lll}-0.81985800 & 8.06340600 & -0.42831500\end{array}$ $\begin{array}{llll}0.36782800 & 9.05608700 & -1.19186500\end{array}$ $\begin{array}{llll}6.86258000 & 2.68939300 & -0.11727800\end{array}$ $\begin{array}{lll}7.37080100 & -0.05056800 & -0.48138600\end{array}$ $\begin{array}{llll}7.62881000 & 2.90679500 & -1.30965500\end{array}$ $\begin{array}{lll}8.13891800 & 1.99089500 & -1.62301000\end{array}$ $\begin{array}{llll}6.98546400 & 3.26592500 & -2.12238200\end{array}$ $\begin{array}{llll}8.36319600 & 3.67596600 & -1.05998100\end{array}$ $\begin{array}{lll}8.11313900 & 0.01574500 & 0.74410800\end{array}$ $\begin{array}{lll}7.60285200 & -0.54709900 & 1.53542600\end{array}$ 


\section{Figure-8 GS (no D3)}

$\begin{array}{llll}\text { C } & -9.35115800 & 2.62843400 & 0.88159200 \\ \text { C } & -9.22503400 & 1.32678400 & 0.40691100 \\ \text { C } & -10.31838400 & 0.50301600 & 0.14003100 \\ \text { C } & -11.61363400 & 1.06577900 & 0.29867500 \\ \text { C } & -11.74168300 & 2.40359600 & 0.71097600 \\ \text { C } & -10.17300500 & -0.86420300 & -0.32311500 \\ \text { C } & -11.32817800 & -1.63971500 & -0.60797600 \\ \text { C } & -12.63141600 & -1.04224800 & -0.43596500 \\ \text { C } & -12.76753400 & 0.25465300 & -0.01143000 \\ \text { C } & -8.93169800 & -1.48587000 & -0.47043400 \\ \text { C } & -8.78201200 & -2.78815100 & -0.93690300 \\ \text { C } & -11.18168400 & -2.97712400 & -1.01198200 \\ \text { C } & -10.64312600 & 3.20633100 & 1.05066300 \\ \text { C } & -8.16358900 & 3.42229000 & 1.16405100 \\ \text { C } & -6.93619700 & 2.79942000 & 1.37335700 \\ \text { C } & -5.74327400 & 3.50283500 & 1.56387900 \\ \text { C } & -5.83513300 & 4.92234900 & 1.60828900 \\ \text { C } & -7.08964800 & 5.54677700 & 1.49085200 \\ \text { C } & -4.45629900 & 2.84989000 & 1.71432900 \\ \text { C } & -3.28183700 & 3.64689400 & 1.84677000 \\ \text { C } & -3.40661100 & 5.08530000 & 1.87359200 \\ \text { C } & -4.63079000 & 5.69444400 & 1.78153100 \\ \text { C } & -4.30378300 & 1.44449900 & 1.70609500 \\ \text { C } & -3.06512500 & 0.85146500 & 1.79799400 \\ \text { C } & -2.01547400 & 3.03193300 & 1.92842000 \\ \text { C } & -8.27192300 & 4.84407600 & 1.22755500 \\ \text { C } & -9.93529400 & -3.58028600 & -1.22808200 \\ \text { C } & -7.45226700 & -3.36007900 & -1.11190500 \\ \text { C } & -6.32371600 & -2.54807100 & -1.21625100 \\ \text { C } & -5.03026300 & -3.05412000 & -1.39493300 \\ \text { C } & -4.90714600 & -4.46483700 & -1.55155100 \\ \text { C } & -6.04863600 & -5.27797500 & -1.48175700 \\ \text { C } & -3.84632500 & -2.21383600 & -1.45860700 \\ \text { C } & -2.57555000 & -2.81718000 & -1.69753800 \\ \text { C } & -2.49302400 & -4.24905700 & -1.87169200 \\ \text { C } & -3.61056700 & -5.03713100 & -1.80453000 \\ \text { C } & -3.88759000 & -0.81012100 & -1.29345000 \\ \text { C } & -2.75195700 & -0.03363300 & -1.37701800 \\ \text { C } & -1.41903600 & -2.01697500 & -1.77234600 \\ \text { C } & -7.32332500 & -4.77566100 & -1.21190500 \\ \text { C } & -8.52857200 & -5.62974400 & -1.21554100 \\ \text { C } & -9.77208300 & -5.01638700 & -1.56095700 \\ \text { C } & -8.49512000 & -7.03062800 & -0.95681000 \\ \text { C } & -9.59613800 & -7.81184500 & -1.34142300 \\ & & & \end{array}$


C $\begin{array}{llll}0.67261200 & 0.87658100 & -1.72045700\end{array}$ $\begin{array}{lll}-8.23236200 & 0.95516200 & 0.19545500\end{array}$ $\begin{array}{llll}-12.73978100 & 2.80436000 & 0.71828500\end{array}$ $\begin{array}{lll}-8.04254500 & -0.94653300 & -0.17426700\end{array}$

$\begin{array}{lll}-12.09134800 & -3.54021800 & -1.11325000\end{array}$ $\begin{array}{lll}-6.91207300 & 1.71894400 & 1.40411300\end{array}$ $\begin{array}{lll}-7.09941200 & 6.61136300 & 1.63785900\end{array}$ $\begin{array}{lll}-5.17405700 & 0.80340400 & 1.61753000\end{array}$ $\begin{array}{lll}-2.97076600 & -0.22844700 & 1.76992900\end{array}$ $\begin{array}{lll}-1.13424200 & 3.65633500 & 2.01603600\end{array}$ $-6.46192900-1.47534200-1.20495800$ $\begin{array}{lll}-5.91194800 & -6.32152500 & -1.70271000\end{array}$ $-4.82897800 \quad-0.31600600-1.08022100$ $\begin{array}{llll}-2.81079200 & 1.03900300 & -1.22928600\end{array}$ $\begin{array}{lll}-0.46210300 & -2.49194100 & -1.95360000\end{array}$ $\begin{array}{lll}8.26979100 & 0.96929800 & 0.01237100\end{array}$ $\begin{array}{lll}12.77535900 & 2.73857300 & -0.74482800\end{array}$ $\begin{array}{lll}8.05783500 & -0.91393100 & 0.38167900\end{array}$ $\begin{array}{lll}12.08240100 & -3.60637900 & 1.12872700\end{array}$ $\begin{array}{llll}6.92738700 & 1.66658000 & -1.18912500\end{array}$

$\begin{array}{lll}7.15480300 & 6.52644600 & -1.79246500\end{array}$

$\begin{array}{llll}5.18252000 & 0.76516700 & -1.14316400\end{array}$

$\begin{array}{llll}2.99093100 & -0.28525400 & -1.27987200\end{array}$

$\begin{array}{lll}1.17207600 & 3.53853600 & -2.05477100\end{array}$

$\begin{array}{lll}6.51876700 & -1.45095000 & 1.48862300\end{array}$

$\begin{array}{lll}5.79045800 & -6.29890500 & 1.45488900\end{array}$

$\begin{array}{lll}4.92908200 & -0.23048400 & 1.64704300\end{array}$

$\begin{array}{lll}2.93204400 & 1.15760900 & 1.81653400\end{array}$

$\begin{array}{llll}0.45518400 & -2.36043400 & 1.79506300\end{array}$

$\begin{array}{lll}1.17032000 & -4.68705400 & 1.64062600\end{array}$

$\begin{array}{lll}3.40231500 & -6.32480200 & 1.35199800\end{array}$

$\begin{array}{lll}2.93901500 & -7.01372600 & 2.52018000\end{array}$

$\begin{array}{lll}2.95389300 & -8.07615200 & 2.26573800\end{array}$

$\begin{array}{llll}3.61211200 & -6.83313700 & 3.36831400\end{array}$

$\begin{array}{lll}1.92321500 & -6.70160900 & 2.78085800\end{array}$

$\begin{array}{llll}0.59956700 & -4.95581600 & 0.35063100\end{array}$

$\begin{array}{llll}0.53214600 & -4.04194400 & -0.24887400\end{array}$

$\begin{array}{lll}1.18921700 & -5.70818800 & -0.18614100\end{array}$

$\begin{array}{lll}-0.40849900 & -5.33303700 & 0.53001200\end{array}$

$\begin{array}{lll}7.31128700 & -7.70023200 & 0.25076600\end{array}$

$\begin{array}{llll}6.70306500 & -6.93633800 & -0.23773500\end{array}$

$\begin{array}{lll}7.72909600 & -8.29316800 & -0.57420600\end{array}$

$\begin{array}{lll}11.98504600 & -5.27242100 & 2.87572500\end{array}$

$\begin{array}{lll}11.81349000 & -4.21227200 & 3.07298200\end{array}$

$\begin{array}{lll}12.05418100 & -5.73718700 & 3.86942700\end{array}$

$13.74473700-1.89092600 \quad 0.75115100$

$\begin{array}{lll}14.03903000 & 0.74004900 & -0.19493500\end{array}$

$14.50555100 \quad-2.29475400-0.39549700$

$13.87295800-2.84536700-1.10332000$

$\begin{array}{llll}14.95165600 & -1.43014400 & -0.89746800\end{array}$

$15.29022100 \quad-2.95506600-0.01848200$

$\begin{array}{lll}14.82373600 & 0.98420900 & 0.97935600\end{array}$

$\begin{array}{lll}15.05956700 & 0.04810900 & 1.49559300\end{array}$

$\begin{array}{lll}15.74186400 & 1.46402600 & 0.63248100\end{array}$

$\begin{array}{lll}14.29421200 & 1.65924100 & 1.66359100\end{array}$

$13.06216800 \quad 4.34839600 \quad-2.55806900$

$\begin{array}{llll}12.69372100 & 3.33795200 & -2.74802900\end{array}$

$\begin{array}{llll}13.28526600 & 4.75468300 & -3.55377500\end{array}$

$\begin{array}{llll}8.77056900 & 7.70334000 & -0.33265100\end{array}$

$\begin{array}{lll}7.98288500 & 7.08010200 & 0.09828600\end{array}$

$\begin{array}{llll}9.25429900 & 8.18281100 & 0.52986600\end{array}$

$\begin{array}{llll}4.80582700 & 6.95728400 & -2.13345700\end{array}$

$2.29918200 \quad 5.70112900-2.25754500$

$\begin{array}{llll}2.20155600 & 6.21600300 & -3.59265100\end{array}$

$\begin{array}{lll}3.00608200 & 6.92989400 & -3.79828700\end{array}$

$\begin{array}{llll}2.23765700 & 5.39926800 & -4.32472600\end{array}$

$\begin{array}{lll}1.23278600 & 6.71752200 & -3.65431600\end{array}$ 


\begin{tabular}{|c|c|c|c|}
\hline $\mathrm{C}$ & 615100 & 7.77745100 & -1.11648500 \\
\hline $\mathrm{H}$ & 4.47872300 & 8.80721300 & -1.36807800 \\
\hline $\mathrm{H}$ & 3.12824000 & 7.65857200 & -1.09538000 \\
\hline $\mathrm{H}$ & 4.63131200 & 7.52739700 & -0.13167700 \\
\hline $\mathrm{O}$ & -2.25090600 & 5.82268200 & 2.01945200 \\
\hline $\mathrm{O}$ & -4.74847800 & 7.06851500 & 1.77986200 \\
\hline C & -1.85577500 & 6.54226600 & 0.84244800 \\
\hline $\mathrm{H}$ & -1.69079900 & 5.85251100 & 0.00503400 \\
\hline $\mathrm{H}$ & -2.61049300 & 7.28569400 & 0.56541700 \\
\hline $\mathrm{H}$ & -0.91558500 & 7.03918900 & 1.09253100 \\
\hline C & -4.45024300 & 7.70409700 & 3.02954100 \\
\hline $\mathrm{H}$ & -4.65021900 & 8.76749800 & 2.87969500 \\
\hline $\mathrm{H}$ & -5.10162800 & 7.31741300 & 3.82349500 \\
\hline $\mathrm{H}$ & -3.40227800 & 7.55217800 & 3.30664700 \\
\hline C & -8.76195200 & 7.75800300 & 0.32177400 \\
\hline $\mathrm{H}$ & -8.00488500 & 7.13977400 & -0.16471300 \\
\hline $\mathrm{H}$ & -9.23018100 & 8.31907700 & -0.49802800 \\
\hline C & -13.06246100 & 4.40347800 & 2.52944900 \\
\hline st & -12.70699000 & 3.38731400 & 2.71096800 \\
\hline r & -13.28357900 & 4.80573700 & 3.52761700 \\
\hline C & -13.72768500 & -1.81387800 & -0.76395700 \\
\hline $\mathrm{C}$ & -14.01139900 & 0.82254500 & 0.17441100 \\
\hline C & -14.54717300 & -2.20787100 & 0.34461200 \\
\hline $\mathrm{H}$ & -13.95585000 & -2.76672900 & 1.08093000 \\
\hline 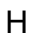 & -15.00468200 & -1.33749300 & 0.82581900 \\
\hline $\mathrm{H}$ & -15.32089300 & -2.85791700 & -0.07064800 \\
\hline C & -14.74348100 & 1.07851400 & -1.03135900 \\
\hline $\mathrm{H}$ & -14.17625800 & 1.74624400 & -1.69207600 \\
\hline $\mathrm{H}$ & -14.97097500 & 0.14635600 & -1.55863400 \\
\hline $\mathrm{H}$ & -15.66865100 & 1.57068300 & -0.72254900 \\
\hline C & -7.39240800 & -7.66771800 & -0.11153800 \\
\hline $\mathrm{H}$ & -6.79419500 & -6.88795800 & 0.36747200 \\
\hline $\mathrm{H}$ & -7.88861000 & -8.19024600 & 0.71842400 \\
\hline C & -11.99506900 & -5.22842500 & -2.84624900 \\
\hline $\mathrm{H}$ & -11.79648900 & -4.18165500 & -3.08532600 \\
\hline $\mathrm{H}$ & -12.10368200 & -5.72246300 & -3.82129500 \\
\hline $\mathrm{O}$ & -1.24395900 & -4.79938800 & -2.07766400 \\
\hline $\mathrm{O}$ & -3.54504800 & -6.40082400 & -2.00720800 \\
\hline C & -2.93812500 & -7.13530800 & -0.93744000 \\
\hline $\mathrm{H}$ & -3.07026000 & -8.19187300 & -1.17994900 \\
\hline $\mathrm{H}$ & -1.87221900 & -6.89818800 & 367100 \\
\hline 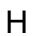 & -3.43951100 & -6.91758800 & 0.01406600 \\
\hline C & -1.02416300 & -5.30381800 & -3.40326000 \\
\hline 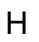 & -1.71590500 & -6.12137200 & 30000 \\
\hline $\mathrm{H}$ & -1.14188300 & -4.50296800 & 385700 \\
\hline $\mathrm{H}$ & 0.00542300 & -5.66856500 & 58600 \\
\hline C & -9.67469200 & -9.29267000 & -1.02258400 \\
\hline 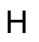 & -9.37574700 & -9.91444100 & -1.87838100 \\
\hline $\mathrm{H}$ & -9.03706200 & -9.57662200 & -0.18357000 \\
\hline $\mathrm{H}$ & -10.69643400 & -9.58789500 & -0.76235000 \\
\hline C & -11.68199900 & -8.15710800 & -2.70006500 \\
\hline $\mathrm{H}$ & -11.19517200 & -9.04392200 & -3.11987600 \\
\hline $\mathrm{H}$ & -12.42315400 & -8.51877800 & -1.97340700 \\
\hline $\mathrm{H}$ & -12.23608100 & -7.68301800 & -3.51234700 \\
\hline C & -6.44296500 & -8.67827100 & -0.79385800 \\
\hline $\mathrm{H}$ & -5.78325100 & -8.16804000 & -1.50506100 \\
\hline $\mathrm{H}$ & -7.01771900 & -9.39682000 & -1.38871000 \\
\hline C & -5.59043700 & -9.43240900 & 0.23524000 \\
\hline $\mathrm{H}$ & -4.88205200 & -10.11608600 & -0.24893700 \\
\hline $\mathrm{H}$ & -5.01131300 & -8.73824100 & 0.85687200 \\
\hline 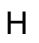 & -6.21992600 & -10.02760500 & 0.90947200 \\
\hline C & -13.34402300 & -5.33766900 & -2.10708700 \\
\hline $\mathrm{H}$ & -13.68824700 & -6.37727800 & -2.11613700 \\
\hline $\mathrm{H}$ & -13.21547600 & -5.08071800 & -1.04737200 \\
\hline 0 & -14.40594400 & -4.42675100 & -2.73412900 \\
\hline 4 & -14.13909000 & -3.37255900 & -2.59145600 \\
\hline & - & & -10 \\
\hline
\end{tabular}

\begin{tabular}{|c|c|c|c|}
\hline $\mathrm{H}$ & -15.39168700 & -4.58660500 & -2.27995400 \\
\hline $\mathrm{C}$ & -14.38736800 & 4.34913900 & 1.73493700 \\
\hline $\mathrm{H}$ & -14.87212900 & 5.33131800 & 1.76205700 \\
\hline $\mathrm{H}$ & -14.17929600 & 4.15927400 & 0.67375400 \\
\hline C & -15.34204200 & 3.27622600 & 2.27209300 \\
\hline $\mathrm{H}$ & -14.92914500 & 2.27455500 & 2.10601700 \\
\hline $\mathrm{H}$ & -15.51443500 & 3.40259100 & 3.34913500 \\
\hline $\mathrm{H}$ & -16.31774200 & 3.32124300 & 1.77222300 \\
\hline C & -8.07042400 & 8.77183400 & 1.26194800 \\
\hline $\mathrm{H}$ & -8.79820800 & 9.52248500 & 1.59001600 \\
\hline $\mathrm{H}$ & -7.74046900 & 8.26660500 & 2.17944900 \\
\hline C & -6.87659500 & 9.45916200 & 0.59183500 \\
\hline $\mathrm{H}$ & -7.17931800 & 9.95643900 & -0.33910500 \\
\hline $\mathrm{H}$ & -6.43672800 & 10.22191400 & 1.24666300 \\
\hline $\mathrm{H}$ & -6.09447900 & 8.72969200 & 0.35282700 \\
\hline C & -13.27528000 & 7.33524400 & 2.48921300 \\
\hline $\mathrm{H}$ & -14.00971400 & 7.61353600 & 1.72031300 \\
\hline $\mathrm{H}$ & -13.79783400 & 6.71750200 & 3.22222300 \\
\hline $\mathrm{H}$ & -12.98503700 & 8.26035000 & 2.99768900 \\
\hline C & -11.38514100 & 8.88747400 & 1.02038200 \\
\hline $\mathrm{H}$ & -11.24923600 & 9.50666500 & 1.91842600 \\
\hline $\mathrm{H}$ & -10.75777700 & 9.31704000 & 0.23744700 \\
\hline $\mathrm{H}$ & -12.42516600 & 9.01618900 & 0.70291500 \\
\hline C & 8.12342100 & 8.81975900 & -1.18196000 \\
\hline $\mathrm{H}$ & 8.89946200 & 9.37854900 & -1.71773300 \\
\hline $\mathrm{H}$ & 7.48270500 & 8.38539200 & -1.95865700 \\
\hline C & 7.29078500 & 9.78190400 & -0.32632300 \\
\hline $\mathrm{H}$ & 6.50279200 & 9.24606200 & 0.21763400 \\
\hline $\mathrm{H}$ & 7.91660700 & 10.28993800 & 0.41888600 \\
\hline $\mathrm{H}$ & 6.80846600 & 10.55370900 & -0.93846100 \\
\hline C & 14.38702300 & 4.26997800 & -1.76287500 \\
\hline $\mathrm{H}$ & 14.88362200 & 5.24724000 & -1.78283600 \\
\hline $\mathrm{H}$ & 14.17296600 & 4.07472200 & -0.70358100 \\
\hline C & 15.33347200 & 3.19442100 & -2.30496300 \\
\hline $\mathrm{H}$ & 6400 & 2.19543100 & -2.18577800 \\
\hline $\mathrm{H}$ & 15.54267100 & 3.35318100 & -3.37092900 \\
\hline $\mathrm{H}$ & 2900 & 836600 & -1.77391200 \\
\hline C & 5400 & 500 & -1.09 \\
\hline $\mathrm{H}$ & 7900 & 9.44 & -2.01266300 \\
\hline $\mathrm{H}$ & 10.72301900 & 9.29295500 & -0.33557100 \\
\hline $\mathrm{H}$ & 12.40025300 & 8.99290800 & -0.77255600 \\
\hline C & 13.26598700 & 7.28820100 & -2.54205600 \\
\hline $\mathrm{H}$ & 14.00914300 & 7.56003000 & -1.77931700 \\
\hline $\mathrm{H}$ & 13.77878700 & 6.67343200 & -3.28423600 \\
\hline $\mathrm{H}$ & 12.97230900 & 8.21655800 & -3.04221900 \\
\hline C & 6.39893700 & -8.62527800 & 1.08962900 \\
\hline $\mathrm{H}$ & 6.96859100 & -9.49627200 & 1.43177800 \\
\hline $\mathrm{H}$ & 6.08017700 & -8.10782800 & 2.00355900 \\
\hline C & 5.16808800 & -9.08385000 & 0.30079400 \\
\hline $\mathrm{H}$ & 5.46532500 & -9.60435400 & -0.61962000 \\
\hline $\mathrm{H}$ & 4.55079200 & -9.77592100 & 0.88749400 \\
\hline $\mathrm{H}$ & 4.54344100 & -8.22695700 & 0.02388300 \\
\hline C & 13.35080600 & -5.44294700 & 2.17799500 \\
\hline $\mathrm{H}$ & 13.62678000 & -6.50295900 & 2.15152600 \\
\hline $\mathrm{H}$ & 13.28117400 & -5.13284900 & 1.12793300 \\
\hline C & 14.45032500 & -4.63003200 & 2.86967300 \\
\hline $\mathrm{H}$ & 14.55568500 & -4.91875100 & 3.92403000 \\
\hline $\mathrm{H}$ & 15.42323600 & -4.77776900 & 2.38509700 \\
\hline $\mathrm{H}$ & 14.22073100 & -3.55797500 & 2.83148000 \\
\hline C & 11.66139300 & -8.18473300 & 2.72156300 \\
\hline $\mathrm{H}$ & 12.37961000 & -8.57743800 & 1.98744600 \\
\hline $\mathrm{H}$ & 12.24226100 & -7.69494300 & 3.50526700 \\
\hline $\mathrm{H}$ & 11.17243600 & -9.05146600 & 3.17858100 \\
\hline C & 9.61788900 & -9.31755700 & 1.07687600 \\
\hline $\mathrm{H}$ & 9.33047600 & -9.92357700 & 1.94793100 \\
\hline $\mathrm{H}$ & 8.95755200 & -9.60466700 & 0.25660200 \\
\hline $\mathrm{H}$ & 10.62939700 & -9.62808900 & 0.79571000 \\
\hline
\end{tabular}


Figure-8 TS (no D3)

\begin{tabular}{|c|c|c|c|}
\hline C & 9.49814900 & 3.06019100 & 0.09049600 \\
\hline C & 9.52157600 & 1.66084500 & 0.13913600 \\
\hline C & 10.69438900 & 0.91901700 & 0.33594300 \\
\hline C & 11.90420900 & 1.64784700 & 0.54871200 \\
\hline C & 11.89030600 & 3.04293500 & 0.45113000 \\
\hline $\mathrm{C}$ & 10.75009100 & -0.54212300 & 0.31631200 \\
\hline C & 11.99714200 & -1.18365800 & 0.58338600 \\
\hline C & 13.17277500 & -0.40264100 & 0.85692300 \\
\hline C & 13.13140500 & 0.95216700 & 0.82623800 \\
\hline C & 9.65495000 & -1.36109000 & 0.01034600 \\
\hline C & 9.72584400 & -2.75962800 & -0.04236100 \\
\hline C & 12.07175900 & -2.57838100 & 0.53671800 \\
\hline C & 10.73338500 & 3.77394400 & 0.18476300 \\
\hline C & 8.24535300 & 3.80816200 & -0.08280000 \\
\hline C & 6.99313600 & 3.20697000 & 0.09471000 \\
\hline C & 5.78006700 & 3.88390900 & -0.10718400 \\
\hline $\mathrm{C}$ & 5.85500900 & 5.23568900 & -0.56703700 \\
\hline $\mathrm{C}$ & 7.10494300 & 5.85131800 & -0.69355300 \\
\hline C & 4.46618800 & 3.30034600 & 0.14559700 \\
\hline C & 3.30646900 & 4.08403900 & -0.13723600 \\
\hline C & 3.43602200 & 5.41466100 & -0.66675200 \\
\hline $\mathrm{C}$ & 4.65697400 & 5.97066000 & -0.85978000 \\
\hline C & 4.26087400 & 2.01184100 & 0.69491300 \\
\hline C & 2.99461800 & 1.52239900 & 0.97384000 \\
\hline C & 2.03090800 & 3.56760400 & 0.13288600 \\
\hline C & 8.30413200 & 5.19693200 & -0.42166300 \\
\hline C & 10.98141500 & -3.39090700 & 0.22728300 \\
\hline C & 8.55710400 & -3.58707700 & -0.38411000 \\
\hline C & 7.28207000 & -3.03527400 & -0.56754700 \\
\hline C & 6.14839600 & -3.79558100 & -0.89369800 \\
\hline C & 000 & -5.204 & 75100 \\
\hline C & 3200 & -5.758 & -0.86709200 \\
\hline C & 4.80665300 & -3.238 & $-1.03 \varepsilon$ \\
\hline $\mathrm{C}$ & 3.71980600 & -4.133 & -1.27717200 \\
\hline C & 3.95845500 & -5.5412 & -1.44940600 \\
\hline C & 5.20977100 & -6.05253800 & -1.35124400 \\
\hline C & 4.50060300 & -1.859 & -0.93620300 \\
\hline C & 3.20031500 & -1.387 & -0.99607800 \\
\hline C & 2.40620100 & -3.64309300 & -1.31816500 \\
\hline C & 8.71884200 & -5.00273300 & -0.52656600 \\
\hline $\mathrm{C}$ & 10.01944800 & -5.63899600 & -0.27691100 \\
\hline $\mathrm{C}$ & 11.12148900 & -4.85041000 & 0.13773700 \\
\hline C & 10.20115800 & -7.03256200 & -0.41293400 \\
\hline $\mathrm{C}$ & 11.41111900 & -7.64320600 & -0.13149900 \\
\hline C & 12.49083800 & -6.86778200 & 0.30740000 \\
\hline C & 12.34043600 & -5.49768700 & 0.43527700 \\
\hline C & 10.77370000 & 5.22382500 & -0.04908100 \\
\hline C & 9.58935600 & 5.90907800 & -0.42231400 \\
\hline C & 11.97802300 & 5.95462800 & 0.03482300 \\
\hline C & 12.03413000 & 7.30646300 & -0.25931300 \\
\hline C & 10.87107400 & 7.97610800 & -0.65767300 \\
\hline C & 9.67451300 & 7.28338000 & -0.73184900 \\
\hline C & -9.47445500 & -3.07412700 & -0.04412700 \\
\hline C & -9.49791500 & -1.67478400 & -0.09282500 \\
\hline $\mathrm{C}$ & -10.67075700 & -0.93298900 & -0.28958300 \\
\hline C & -11.88058500 & -1.66184800 & -0.50219100 \\
\hline C & -11.86664400 & -3.05693200 & -0.40456000 \\
\hline C & -10.72647600 & 0.52815000 & -0.27004400 \\
\hline C & -11.97356000 & 1.16965400 & -0.53703600 \\
\hline 0 & -13.14921700 & 0.38860300 & -0.81038600 \\
\hline C & -13.10782500 & -0.96620200 & -0.77961700 \\
\hline 0 & -963131600 & 134714900 & 0.03576000 \\
\hline C & -9.70221800 & 2.74568900 & 0.08837600 \\
\hline & -12.04818100 & 2.56438300 & -0.4904 \\
\hline
\end{tabular}

$\begin{array}{lll}-10.70967900 & -3.78791300 & -0.13830400\end{array}$ $\begin{array}{lll}-8.22163600 & -3.82205000 & 0.12919500\end{array}$ $\begin{array}{lll}-6.96943400 & -3.22083000 & -0.04834200\end{array}$ $\begin{array}{lll}-5.75634800 & -3.89771400 & 0.15363200\end{array}$ $\begin{array}{lll}-5.83125400 & -5.24943600 & 0.61366000\end{array}$ $\begin{array}{lll}-7.08117200 & -5.86509700 & 0.74020600\end{array}$ $\begin{array}{lll}-4.44248400 & -3.31415300 & -0.09923400\end{array}$ $\begin{array}{llll}-3.28274500 & -4.09778100 & 0.18369500\end{array}$ $\begin{array}{lll}-3.41226200 & -5.42832500 & 0.71342000\end{array}$ $\begin{array}{lll}-4.63320000 & -5.98432800 & 0.90652900\end{array}$ $-4.23720500-2.02572300-0.64874000$ $-2.97096500-1.53629900 \quad-0.92777100$ $-2.00720000-3.58137000-0.08655200$ $\begin{array}{lll}-8.28037200 & -5.21079600 & 0.46815900\end{array}$ $\begin{array}{lll}-10.95780900 & 3.37694500 & -0.18123300\end{array}$ $\begin{array}{lll}-8.53346500 & 3.57316500 & 0.43001200\end{array}$ $\begin{array}{lll}-7.25842800 & 3.02137300 & 0.61345400\end{array}$ $\begin{array}{lll}-6.12474500 & 3.78170500 & 0.93951200\end{array}$ $\begin{array}{lll}-6.30483900 & 5.19084600 & 1.09546900\end{array}$ $\begin{array}{lll}-7.57532700 & 5.74458200 & 0.91280700\end{array}$ $\begin{array}{lll}-4.78300200 & 3.22446100 & 1.08461900\end{array}$ $\begin{array}{llll}-3.69614400 & 4.11978500 & 1.32290100\end{array}$ $\begin{array}{lll}-3.93478300 & 5.52744000 & 1.49503700\end{array}$ $\begin{array}{lll}-5.18610000 & 6.03869300 & 1.39686900\end{array}$ $\begin{array}{lll}-4.47696000 & 1.84602700 & 0.98210300\end{array}$ $\begin{array}{lll}-3.17667500 & 1.37372600 & 1.04199000\end{array}$ $\begin{array}{lll}-2.38254300 & 3.62922800 & 1.36391200\end{array}$ $\begin{array}{lll}-8.69519600 & 4.98882900 & 0.57236900\end{array}$ $\begin{array}{lll}-2.09414800 & 2.27358200 & 1.19439200\end{array}$ $\begin{array}{lll}-9.99580600 & 5.62507800 & 0.32270500\end{array}$ $\begin{array}{llll}-11.09786600 & 4.83646100 & -0.09184300\end{array}$ $\begin{array}{lll}-10.17750100 & 7.01865800 & 0.45861000\end{array}$ $\begin{array}{lll}-11.38746000 & 7.62928700 & 0.17713900\end{array}$ $\begin{array}{llll}-12.46718800 & 6.85383500 & -0.26168600\end{array}$ $\begin{array}{llll}-12.31680400 & 5.48372600 & -0.38944000\end{array}$ $\begin{array}{llll}-10.74992800 & -5.23780700 & 0.09548300\end{array}$ $\begin{array}{lll}-9.56555900 & -5.92301200 & 0.46872500\end{array}$ $\begin{array}{lll}-11.95420000 & -5.96868000 & 0.01146600\end{array}$ $\begin{array}{lll}-12.01023600 & -7.32053800 & 0.30551600\end{array}$ $\begin{array}{lll}-10.84715600 & -7.99014100 & 0.70387600\end{array}$ $\begin{array}{lll}-9.65063800 & -7.29734500 & 0.77814200\end{array}$ $\begin{array}{lll}1.84600700 & 2.30250900 & 0.69493100\end{array}$ $\begin{array}{llll}0.49738700 & 1.93140100 & 0.97658300\end{array}$ $\begin{array}{lll}-0.71403400 & 1.92429100 & 1.10335200\end{array}$ $\begin{array}{llll}2.11779300 & -2.28746200 & -1.14855200\end{array}$ $-1.82233400-2.31635800-0.64879700$ $\begin{array}{llll}0.73767800 & -1.93819300 & -1.05745100\end{array}$ $\begin{array}{lll}-0.47373000 & -1.94527800 & -0.93057300\end{array}$ $\begin{array}{llll}8.59167300 & 1.12684300 & -0.01186000\end{array}$ $\begin{array}{lll}12.84195700 & 3.54950100 & 0.56571400\end{array}$ $8.71578800 \quad-0.88199700 \quad-0.23310900$ $\begin{array}{lll}13.04338200 & -3.01994700 & 0.72552900\end{array}$ $\begin{array}{lll}6.96039500 & 2.17893700 & 0.43389600\end{array}$ $\begin{array}{llll}7.10669200 & 6.89344600 & -0.99219900\end{array}$ $\begin{array}{llll}5.11478200 & 1.38613400 & 0.93509300\end{array}$ $\begin{array}{lll}2.87692600 & 0.53824200 & 1.41565800\end{array}$ $\begin{array}{llll}1.15860700 & 4.17712900 & -0.08508900\end{array}$ $\begin{array}{llll}7.15153400 & -1.97125800 & -0.41934700\end{array}$ $\begin{array}{llll}7.67968100 & -6.83371800 & -0.97624400\end{array}$ $\begin{array}{llll}5.29941100 & -1.13737100 & -0.79976900\end{array}$ $\begin{array}{llll}3.00360500 & -0.32568300 & -0.90261700\end{array}$ $\begin{array}{llll}1.58704000 & -4.34196700 & -1.46228500\end{array}$ $\begin{array}{lll}-8.56801500 & -1.14075800 & 0.05809100\end{array}$ $-12.81830000-3.56351800-0.51901000$ $\begin{array}{lll}-8.69212900 & 0.86808300 & 0.27916500\end{array}$ $\begin{array}{lll}-13.01982900 & 3.00592400 & -0.67921600\end{array}$ $\begin{array}{lll}-6.93672000 & -2.19282500 & -0.38761100\end{array}$ 


\begin{tabular}{|c|c|c|c|}
\hline $\mathrm{H}$ & -7.08289600 & -6.90718100 & 00 \\
\hline $\mathrm{H}$ & -5.09113100 & -1.40006600 & -0.88898700 \\
\hline $\mathrm{H}$ & -2.85330000 & -0.55220100 & -1.36972800 \\
\hline $\mathrm{H}$ & -1.13488300 & -4.19084900 & 0.13148400 \\
\hline H & -7.12789600 & 1.95734600 & 0.46534300 \\
\hline $\mathrm{H}$ & -7.65602700 & 6.81984900 & 1.02187900 \\
\hline $\mathrm{H}$ & -5.27577500 & 1.12348800 & 0.84573300 \\
\hline $\mathrm{H}$ & -2.97997600 & 0.31179600 & 0.94860000 \\
\hline $\mathrm{H}$ & -1.56337500 & 4.32810500 & 1.50798100 \\
\hline $\mathrm{O}$ & 2.83942400 & -6.38941100 & -1.72023600 \\
\hline 0 & 5.44364900 & -7.45341200 & -1.51783200 \\
\hline C & 2.49727500 & -7.11891500 & -0.53885700 \\
\hline $\mathrm{H}$ & 1.44156900 & -7.29219400 & -0.51988100 \\
\hline $\mathrm{H}$ & 3.01271200 & -8.05658200 & -0.53686100 \\
\hline $\mathrm{H}$ & 2.78152900 & -6.55382000 & 0.32414300 \\
\hline $\mathrm{C}$ & 5.35128200 & -7.79122700 & -2.90428500 \\
\hline $\mathrm{H}$ & 6.18103900 & -8.40939600 & -3.17681400 \\
\hline & 4.43855600 & -8.32048000 & -3.08239200 \\
\hline & 5.36513900 & -6.89657700 & -3.49106300 \\
\hline & 9.03536700 & -7.92061700 & -0.88610100 \\
\hline & 8.67286000 & -7.56202900 & -1.82679400 \\
\hline & 9.37682000 & -8.92867300 & -0.99625700 \\
\hline $\mathrm{C}$ & 13.54953800 & -4.67870000 & 0.92408200 \\
\hline & 13.87085000 & -4.01807500 & 0.14611400 \\
\hline & 13.26903200 & -4.10719700 & 1.78408200 \\
\hline $\mathrm{C}$ & 14.39566900 & -1.09078300 & 1.13240400 \\
\hline & 14.31036700 & 1.72535600 & 1.06525200 \\
\hline $\mathrm{C}$ & 14.51175600 & -1.31132200 & 2.54051900 \\
\hline 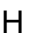 & 15.09908700 & -2.18813700 & 2.71698200 \\
\hline TI & 14.98567600 & -0.46697200 & 2.99590000 \\
\hline TI & 13.53736800 & -1.44387700 & 2.96229800 \\
\hline C & 15.36658000 & 1.25838300 & 0.22189700 \\
\hline $\mathrm{H}$ & 15.93856300 & 2.09215500 & -0.12819000 \\
\hline $\mathrm{H}$ & 16.00059800 & 0.59858800 & 0.77650600 \\
\hline $\mathrm{H}$ & 14.95089400 & 0.73499200 & -0.61366700 \\
\hline C & 13.27959400 & 5.24844600 & 0.45769800 \\
\hline $\mathrm{H}$ & 13.12766000 & 4.75035200 & 1.39242800 \\
\hline $\mathrm{H}$ & 14.06048300 & 5.97246300 & 0.56215500 \\
\hline C & 8.41320100 & 8.05744800 & -1.15788800 \\
\hline $\mathrm{H}$ & 7.96338300 & 7.57148300 & -1.99836600 \\
\hline $\mathrm{H}$ & 7.71763600 & 8.08096000 & -0.34515300 \\
\hline $\mathrm{O}$ & 4.78276100 & 7.30708700 & -1.35277100 \\
\hline 0 & 2.24267200 & 6.14784900 & -0.95528700 \\
\hline $\mathrm{O}$ & 5.07298600 & 8.19055300 & -0.26642000 \\
\hline & 5.75485400 & 8.94712800 & -0.59437100 \\
\hline & 4.16741000 & 8.64904000 & 0.07214000 \\
\hline $\mathrm{H}$ & 5.51385400 & 7.63654600 & 0.53583300 \\
\hline C & 2.15177100 & 6.37513400 & -2.36417900 \\
\hline $\mathrm{H}$ & 1.14895000 & 6.19438700 & -2.69064600 \\
\hline$H$ & 2.42001300 & 7.38821900 & -2.58006300 \\
\hline 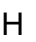 & 2.81833100 & 5.71286500 & -2.87603500 \\
\hline & -2.81574100 & 6.37558100 & 1.76578200 \\
\hline 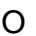 & -5.41997100 & 7.43958000 & 1.56336100 \\
\hline & -2.33325700 & 6.12072200 & 3.08758200 \\
\hline & -1.36129400 & 5.67663700 & 3.03303200 \\
\hline & -2.27482000 & 7.04141100 & 3.62963300 \\
\hline t & -3.00263800 & 5.45342200 & 3.58911900 \\
\hline C & -6.04622600 & 7.96124500 & 0.38838500 \\
\hline $\mathrm{H}$ & -7.04991000 & 8.25212100 & 0.61840700 \\
\hline & -5.49903100 & 8.81241700 & 0.04055700 \\
\hline & -6.05833400 & 7.20953500 & -0.37298600 \\
\hline & -9.01168500 & 7.90673300 & 0.93168000 \\
\hline & -8.59852800 & 7.50306200 & 1.83237500 \\
\hline & -8.25604700 & 7.93650500 & 0.17469500 \\
\hline & -13.52591100 & 4.66471800 & -0.87819800 \\
\hline & -13.24544500 & 4.09326200 & -1.73824200 \\
\hline & -13.84714600 & ) & -0 \\
\hline
\end{tabular}

C $\begin{array}{lll}-14.28680200 & -1.73942300 & -1.01845000\end{array}$

$\begin{array}{lll}-14.37215000 & 1.07671400 & -1.08577200\end{array}$

$\begin{array}{lll}-14.42737300 & -1.97303900 & -2.42221800\end{array}$

$-14.62534800 \quad-3.01059400 \quad-2.59305400$

$\begin{array}{lll}-15.23887300 & -1.38790000 & -2.80166700\end{array}$

$\begin{array}{lll}-13.52308100 & -1.69542700 & -2.92230500\end{array}$

$\begin{array}{lll}-15.10282300 & 1.25544400 & 0.13039800\end{array}$

$\begin{array}{lll}-15.66260500 & 2.16592200 & 0.07965400\end{array}$

$\begin{array}{lll}-15.77238100 & 0.43232300 & 0.26847300\end{array}$

$\begin{array}{lll}-14.42021100 & 1.30182300 & 0.95307100\end{array}$

$\begin{array}{lll}-8.38928200 & -8.07138200 & 1.20410700\end{array}$

$\begin{array}{lll}-7.93919400 & -7.58517100 & 2.04429800\end{array}$

$\begin{array}{lll}-8.65831600 & -9.07154700 & 1.47279300\end{array}$

$\begin{array}{lll}-13.25578400 & -5.26256800 & -0.41147700\end{array}$

$\begin{array}{llll}-13.52237700 & -4.53445600 & 0.32587000\end{array}$

$\begin{array}{lll}-13.10869400 & -4.77821000 & -1.35416600\end{array}$

$\begin{array}{llll}-4.75895300 & -7.32068400 & 1.39972000\end{array}$

$\begin{array}{lll}-2.21889300 & -6.16143600 & 1.00206700\end{array}$

$\begin{array}{lll}-4.30289200 & -7.37386100 & 2.75400300\end{array}$

$\begin{array}{lll}-4.94384900 & -8.01597700 & 3.32124200\end{array}$

$\begin{array}{lll}-3.30341300 & -7.75506200 & 2.77906200\end{array}$

$\begin{array}{lll}-4.32016500 & -6.39033500 & 3.17504700\end{array}$

$-1.88009400-6.98131100-0.11950400$

$-1.59850400 \quad-6.35966500 \quad-0.94361700$

$\begin{array}{lll}-1.06269700 & -7.62104300 & 0.14031200\end{array}$

$\begin{array}{lll}-2.72557400 & -7.57669600 & -0.39442200\end{array}$

$\begin{array}{lll}-11.54477900 & 9.15193400 & 0.34565800\end{array}$

$\begin{array}{lll}-11.32533500 & 9.42339400 & 1.35711900\end{array}$

$\begin{array}{llll}-10.86879900 & 9.65545300 & -0.31344700\end{array}$

$\begin{array}{lll}-12.54950800 & 9.43489600 & 0.11038900\end{array}$

$\begin{array}{llll}-13.81344900 & 7.52067100 & -0.60008100\end{array}$

$\begin{array}{lll}-14.13263100 & 7.20679100 & -1.57193600\end{array}$

$\begin{array}{lll}-14.54572700 & 7.23424400 & 0.12561100\end{array}$

$\begin{array}{llll}-13.69737800 & 8.58430000 & -0.58903600\end{array}$

$\begin{array}{llll}-14.67634400 & 5.61904900 & -1.24881700\end{array}$

$\begin{array}{llll}-14.98070000 & 6.16497500 & -0.38035100\end{array}$

$\begin{array}{lll}-14.34396600 & 6.30291000 & -2.00165100\end{array}$

$\begin{array}{llll}-15.86710500 & 4.80340500 & -1.78585800\end{array}$

$\begin{array}{llll}-15.53070700 & 4.15208300 & -2.56529100\end{array}$

$\begin{array}{llll}-16.28816900 & 4.22218400 & -0.99226700\end{array}$

$\begin{array}{lll}-16.60978400 & 5.46923500 & -2.17315400\end{array}$

$\begin{array}{lll}-9.52805800 & 9.33294400 & 1.19791500\end{array}$

$\begin{array}{lll}-9.82484700 & 9.78313500 & 0.27371600\end{array}$

$\begin{array}{lll}-10.36769600 & 9.29050900 & 1.85981300\end{array}$

$\begin{array}{lll}-8.40882700 & 10.17229000 & 1.84167000\end{array}$

$\begin{array}{lll}-7.99369700 & 9.63758300 & 2.67031600\end{array}$

$\begin{array}{lll}-7.64286600 & 10.35996600 & 1.11849600\end{array}$

$\begin{array}{lll}-8.81227200 & 11.10250400 & 2.18348100\end{array}$

$\begin{array}{lll}-14.38537400 & -6.30150700 & -0.53888300\end{array}$

$\begin{array}{lll}-14.31940200 & -6.78514800 & -1.49105900\end{array}$

$\begin{array}{lll}-14.28912200 & -7.03019400 & 0.23871000\end{array}$

$\begin{array}{lll}-15.74844200 & -5.59560200 & -0.41502100\end{array}$

$\begin{array}{lll}-15.72096800 & -4.90825600 & 0.40455200\end{array}$

$\begin{array}{lll}-15.95824400 & -5.06390200 & -1.31955400\end{array}$

$\begin{array}{lll}-16.51318100 & -6.32418000 & -0.24400300\end{array}$

$\begin{array}{lll}-7.38850700 & -8.10571100 & 0.03411900\end{array}$

$\begin{array}{lll}-7.49059500 & -7.21427600 & -0.54882300\end{array}$

$\begin{array}{lll}-6.39202100 & -8.16811300 & 0.41885300\end{array}$

$\begin{array}{lll}-7.67499900 & -9.33322800 & -0.85059800\end{array}$

$-7.84442400-10.18834000-0.23013100$

$\begin{array}{lll}-8.54356200 & -9.14785800 & -1.44737100\end{array}$

$\begin{array}{lll}-6.83606900 & -9.51637100 & -1.48899700\end{array}$

$\begin{array}{lll}-13.34377700 & -8.08281300 & 0.19503400\end{array}$

$\begin{array}{lll}-14.03628400 & -7.69596800 & 0.91314600\end{array}$

$\begin{array}{lll}-13.74459500 & -7.95949800 & -0.78936300\end{array}$

$\begin{array}{lll}-13.17700200 & -9.12260500 & 0.38455600\end{array}$ $\begin{array}{lll}-10.89819800 & -9.48816000 & 1.05733700\end{array}$ 


\begin{tabular}{lccc}
$\mathrm{H}$ & -10.70554900 & -10.06855800 & 0.17931300 \\
$\mathrm{H}$ & -10.15722600 & -9.70528600 & 1.79809200 \\
$\mathrm{H}$ & -11.86728200 & -9.73146800 & 1.44019100 \\
$\mathrm{C}$ & 7.89987600 & -7.87392500 & 0.15316800 \\
$\mathrm{H}$ & 7.85843800 & -6.89921000 & 0.59261400 \\
$\mathrm{H}$ & 6.96800100 & -8.08867300 & -0.32682500 \\
$\mathrm{C}$ & 8.16522800 & -8.92101900 & 1.25079400 \\
$\mathrm{H}$ & 8.45432000 & -9.84657000 & 0.79837800 \\
$\mathrm{H}$ & 8.95056700 & -8.57605200 & 1.89043600 \\
$\mathrm{H}$ & 7.27516600 & -9.06796000 & 1.82620500 \\
$\mathrm{C}$ & 14.69991100 & -5.63305600 & 1.29482200 \\
$\mathrm{H}$ & 14.52409000 & -6.04264300 & 2.26756300 \\
$\mathrm{H}$ & 14.75080100 & -6.42574600 & 0.57791700 \\
$\mathrm{C}$ & 16.03009000 & -4.85703000 & 1.29734800 \\
$\mathrm{H}$ & 16.07476100 & -4.22300400 & 0.43658300 \\
$\mathrm{H}$ & 16.09332300 & -4.26050700 & 2.18338500 \\
$\mathrm{H}$ & 16.84640200 & -5.54839200 & 1.27383100 \\
$\mathrm{C}$ & 11.56845000 & -9.16583800 & -0.30013800 \\
$\mathrm{H}$ & 11.06151700 & -9.66731000 & 0.49763700 \\
$\mathrm{H}$ & 11.14591500 & -9.46657600 & -1.23604400 \\
$\mathrm{H}$ & 12.60723100 & -9.42155900 & -0.27917700 \\
$\mathrm{C}$ & 13.83710500 & -7.53463300 & 0.64573800 \\
$\mathrm{H}$ & 14.58670800 & -7.19089500 & -0.03605400 \\
$\mathrm{H}$ & 14.12027600 & -7.27937400 & 1.64551700 \\
$\mathrm{H}$ & 13.73972700 & -8.59696300 & 0.56283000 \\
$\mathrm{C}$ & 13.67593800 & 4.21710500 & -0.61507700 \\
$\mathrm{H}$ & 14.18408600 & 3.39752700 & -0.15141900 \\
$\mathrm{H}$ & 12.79621000 & 3.85870700 & -1.10754800 \\
$\mathrm{C}$ & 14.60708200 & 4.88117000 & -1.64638100 \\
$\mathrm{H}$ & 14.04090000 & 5.55196700 & -2.25823800 \\
$\mathrm{H}$ & 15.37419800 & 5.42522000 & -1.13604400 \\
$\mathrm{H}$ & 15.05311300 & 4.12771900 & -2.26141700 \\
$\mathrm{C}$ & 8.80038300 & 9.49710400 & -1.54399800 \\
$\mathrm{H}$ & 9.53957800 & 9.86268400 & -0.86220500 \\
$\mathrm{H}$ & 9.19664800 & 9.50550300 & -2.53788100 \\
$\mathrm{C}$ & 7.55335000 & 10.39850900 & -1.48093100 \\
$\mathrm{H}$ & 6.83570900 & 10.06544900 & -2.20132200 \\
$\mathrm{H}$ & 7.12558800 & 10.34754600 & -0.50148100 \\
$\mathrm{H}$ & 7.83230800 & 11.40883300 & -1.69617100 \\
$\mathrm{C}$ & 10.92219200 & 9.47410100 & -1.01123500 \\
$\mathrm{H}$ & 10.52977100 & 9.62181300 & -1.99565700 \\
$\mathrm{H}$ & 10.33625200 & 10.02697300 & -0.30702900 \\
$\mathrm{H}$ & 11.93607100 & 9.81432800 & -0.97667600 \\
$\mathrm{H}$ & 13.36771900 & 8.06866600 & -0.14892700 \\
\hline & 13.97234600 & 7.62120100 & 0.61206000 \\
\hline 13.88265600 & 8.02402200 & -1.08580900 \\
\hline & 13.17474100 & 9.09036000 & 0.10366500
\end{tabular}

\section{Helicene 3 GS (no D3)}

$\begin{array}{lrrr}\text { C } & -0.92870900 & -2.55248600 & -0.18664100 \\ \text { C } & -1.63562500 & -1.34404600 & -0.18393000 \\ \text { C } & -3.02133300 & -1.27201600 & -0.31819800 \\ \text { C } & -3.78228000 & -2.47467100 & -0.44024300 \\ \text { C } & -3.08762600 & -3.68588200 & -0.37310700 \\ \text { C } & -3.72101900 & 0.01214900 & -0.29676400 \\ \text { C } & -5.12483100 & 0.02453000 & -0.03384800 \\ \text { C } & -5.86652200 & -1.24486600 & 0.09267600 \\ \text { C } & -5.25898600 & -2.42110700 & -0.43179800 \\ \text { C } & -3.05572100 & 1.21113800 & -0.54836300 \\ \text { C } & -3.69347100 & 2.46005300 & -0.52951200 \\ \text { C } & -5.77958900 & 1.25666500 & -0.08694700 \\ \text { C } & -2.99692500 & 3.71769000 & -0.74636900 \\ \text { C } & -1.68965000 & -3.75299000 & -0.27032800 \\ \text { C } & 0.51879000 & -2.62959100 & -0.05972000 \\ \text { C } & 1.34725800 & -1.50090400 & -0.03254200 \\ \text { C } & 2.73298300 & -1.57645100 & 0.09940700\end{array}$

C C C C $\begin{array}{llll}3.36319300 & -2.85478400 & 0.18508700\end{array}$ $\begin{array}{lll}2.54709700 & -3.98574700 & 0.09117800\end{array}$ $\begin{array}{llll}3.56618800 & -0.37441000 & 0.11621900\end{array}$ $\begin{array}{llll}4.96390100 & -0.50254800 & -0.15219900\end{array}$ $\begin{array}{llll}5.56578000 & -1.83946100 & -0.33177700\end{array}$ $\begin{array}{lll}4.83564800 & -2.95649500 & 0.16389700\end{array}$ $\begin{array}{lll}3.03206400 & 0.87801900 & 0.41558600\end{array}$ $\begin{array}{lll}3.80046100 & 2.05098600 & 0.44600600\end{array}$ $\begin{array}{lll}5.74643800 & 0.64915400 & -0.05192100\end{array}$ $\begin{array}{llll}3.24462600 & 3.36608600 & 0.72058700\end{array}$ $1.14964500 \quad-3.90555400-0.00823300$ $\begin{array}{lll}-5.10034200 & 2.46636200 & -0.29956000\end{array}$ $\begin{array}{lll}5.19998800 & 1.91468600 & 0.21299300\end{array}$ $\begin{array}{lll}4.11438400 & 4.49606200 & 0.76143500\end{array}$ $\begin{array}{llll}-3.73800000 & 4.93641400 & -0.73264200\end{array}$ $\begin{array}{lll}3.59631300 & 5.78100300 & 1.01952700\end{array}$ $\begin{array}{lll}1.86853700 & 3.59105100 & 0.95507400\end{array}$ $\begin{array}{lll}-1.60357300 & 3.80178400 & -0.97178300\end{array}$ $\begin{array}{lll}-1.70630100 & 6.22440700 & -1.14399200\end{array}$ $\begin{array}{llll}-3.08130700 & 6.16823500 & -0.92599800\end{array}$ $\begin{array}{lll}-1.01620000 & -5.02761400 & -0.21151600\end{array}$ $\begin{array}{llll}5.52796200 & 4.31534900 & 0.53434800\end{array}$ $\begin{array}{lll}1.37283000 & 4.85051500 & 1.21975100\end{array}$ $\begin{array}{llll}-0.97185200 & 5.01095600 & -1.17321100\end{array}$ $\begin{array}{lll}0.34632500 & -5.10076500 & -0.09940000\end{array}$ $\begin{array}{lll}6.04717800 & 3.07736800 & 0.27081400\end{array}$ $\begin{array}{llll}2.23606100 & 5.97597100 & 1.24901700\end{array}$ $\begin{array}{lll}-5.16423100 & 4.89979700 & -0.51556700\end{array}$ $\begin{array}{llll}-5.81635700 & 3.71537800 & -0.30722300\end{array}$ $\begin{array}{lll}-1.08895800 & -0.42432700 & -0.02080200\end{array}$ $\begin{array}{lll}-3.61867000 & -4.62440100 & -0.35993200\end{array}$ $\begin{array}{lll}-2.00537600 & 1.16914100 & -0.80603900\end{array}$ $\begin{array}{lll}-6.85464500 & 1.31379700 & -0.01112500\end{array}$ $\begin{array}{lll}0.89842600 & -0.52514800 & -0.16800600\end{array}$ $\begin{array}{lll}2.97921300 & -4.97383900 & 0.05118000\end{array}$ $\begin{array}{llll}1.98327800 & 0.93713900 & 0.67685300\end{array}$ $\begin{array}{lll}6.82087800 & 0.59359800 & -0.12919700\end{array}$ $\begin{array}{llll}4.27568100 & 6.62498400 & 1.02789600\end{array}$ $\begin{array}{lll}1.17009100 & 2.76135700 & 0.92453600\end{array}$ $\begin{array}{lll}-1.00029100 & 2.90014900 & -0.98362800\end{array}$ $\begin{array}{lll}-3.66411500 & 7.08100000 & -0.89108500\end{array}$ $\begin{array}{lll}0.31149000 & 4.99514300 & 1.38840200\end{array}$ $\begin{array}{llll}0.09986000 & 5.04645700 & -1.33411900\end{array}$ $\begin{array}{lll}1.69252700 & 7.27248800 & 1.49589500\end{array}$ $\begin{array}{lll}-1.02168900 & 7.46425100 & -1.32024400\end{array}$ $\begin{array}{lll}1.16358400 & 8.34360200 & 1.70083600\end{array}$ $\begin{array}{llll}-0.37616200 & 8.47995000 & -1.46399000\end{array}$ $\begin{array}{llll}0.48959400 & 9.61713100 & 1.92718100\end{array}$ $\begin{array}{lll}0.18426600 & 9.72791100 & 2.97532500\end{array}$ $\begin{array}{lll}1.13667900 & 10.46704200 & 1.67776400\end{array}$ $\begin{array}{lll}-0.41204800 & 9.68489500 & 1.30581800\end{array}$ $\begin{array}{llll}0.43582300 & 9.68173500 & -1.61739300\end{array}$ $\begin{array}{lll}1.33444100 & 9.61387400 & -0.99172500\end{array}$ $\begin{array}{lll}0.75926800 & 9.81655100 & -2.65724200\end{array}$ $\begin{array}{lll}-0.11542000 & 10.58261100 & -1.32106600\end{array}$ $\begin{array}{lll}5.53796800 & -4.13915900 & 0.51430300\end{array}$ $\begin{array}{lll}6.86145600 & -4.29538600 & 0.07876500\end{array}$ $\begin{array}{lll}7.49715600 & -3.27850200 & -0.66541700\end{array}$ $6.86008200-2.04585300-0.87723100$ $-6.08265200-3.51083700-0.81881900$ $\begin{array}{lll}-7.42961500 & -3.51790700 & -0.42340800\end{array}$ $\begin{array}{lll}-7.18119400 & -1.32412900 & 0.62187300\end{array}$ $\begin{array}{lll}-7.95865400 & -2.45648500 & 0.34101900\end{array}$ $\begin{array}{lll}6.32858400 & 5.43684500 & 0.55509500\end{array}$ $\begin{array}{lll}7.40004200 & 2.90032500 & 0.06982700\end{array}$ $\begin{array}{lll}7.22599500 & 5.50658200 & 1.67220300\end{array}$ $\begin{array}{lll}6.66730500 & 5.49538300 & 2.61682600\end{array}$ 


\begin{tabular}{|c|c|c|c|}
\hline $\mathrm{H}$ & 7.93893400 & 4.67625400 & 1.65612300 \\
\hline $\mathrm{H}$ & 7.75671200 & 6.45661700 & 1.57457600 \\
\hline C & 7.87229100 & 3.38496300 & -1.19371300 \\
\hline $\mathrm{H}$ & 7.72207600 & 4.46641900 & -1.27910300 \\
\hline $\mathrm{H}$ & 8.93926800 & 3.15244000 & -1.22880600 \\
\hline $\mathrm{H}$ & 7.36008300 & 2.87394500 & -2.01937400 \\
\hline C & 7.52623400 & -1.01337000 & -1.76917000 \\
\hline H & 6.79609300 & -0.31491600 & -2.18278900 \\
\hline $\mathrm{H}$ & 8.01825700 & -1.51058800 & -2.61195400 \\
\hline C & 4.93954100 & -5.15948400 & 1.47116300 \\
\hline $\mathrm{H}$ & 4.08187800 & -4.74532500 & 2.00352800 \\
\hline $\mathrm{H}$ & 5.68720400 & -5.43030500 & 2.22380200 \\
\hline $\mathrm{O}$ & 1.00113500 & -6.31320400 & -0.03715800 \\
\hline 0 & -1.79284500 & -6.16301700 & -0.30575200 \\
\hline 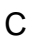 & 0.96636600 & -7.07176900 & -1.25384200 \\
\hline $\mathrm{H}$ & -0.05951500 & -7.34672700 & -1.51832100 \\
\hline $\mathrm{H}$ & 1.41795900 & -6.50225800 & -2.07625400 \\
\hline $\mathrm{H}$ & 1.55752600 & -7.97130800 & -1.06607800 \\
\hline $\mathrm{C}$ & -1.86300900 & -6.93674200 & 0.90030500 \\
\hline $\mathrm{H}$ & -2.26761100 & -6.33323700 & 1.72253300 \\
\hline$\Pi$ & -2.54332000 & -7.76496300 & 0.68867400 \\
\hline $\mathrm{H}$ & -0.87706800 & -7.32334500 & 1.17810400 \\
\hline C & -5.57688900 & -4.61092600 & -1.73448200 \\
\hline $\mathrm{H}$ & -4.68761900 & -4.29679400 & -2.28371900 \\
\hline $\mathrm{H}$ & -6.34633500 & -4.86145100 & -2.47264200 \\
\hline C & -7.70407200 & -0.28636700 & 1.60464100 \\
\hline $\mathrm{H}$ & -6.88870300 & 0.31159000 & 2.01525400 \\
\hline $\mathrm{H}$ & -8.18735500 & -0.79550000 & 2.44483500 \\
\hline 0 & -7.18221300 & 3.67607100 & -0.11546200 \\
\hline $\mathrm{O}$ & -5.83981100 & 6.10101000 & -0.48888600 \\
\hline C & -7.60930900 & 4.17234600 & 1.15993000 \\
\hline $\mathrm{H}$ & -7.16030200 & 3.58651300 & 1.97258600 \\
\hline $\mathrm{H}$ & -7.34584200 & 5.22854500 & 1.27759300 \\
\hline $\mathrm{H}$ & -8.69539400 & 4.05444500 & 1.18389400 \\
\hline C & -6.70456100 & 6.31922500 & -1.61206100 \\
\hline $\mathrm{H}$ & -7.50014200 & 5.56803500 & -1.64705100 \\
\hline $\mathrm{H}$ & -7.13595700 & 7.31342800 & 0400 \\
\hline $\mathrm{H}$ & -6.13340600 & 6.29602800 & 00600 \\
\hline C & -8.73392875 & 0.68734921 & 5258 \\
\hline $\mathrm{H}$ & -8.60158354 & 1.65786135 & 3918 \\
\hline $\mathrm{H}$ & -8.59327715 & 0.74496116 & -0.05689707 \\
\hline C & -10.15669641 & 0.18133500 & 1.30438136 \\
\hline $\mathrm{H}$ & -10.51169336 & -0.39907560 & 0.47854803 \\
\hline $\mathrm{H}$ & -10.13985852 & -0.42531385 & 2.18562688 \\
\hline $\mathrm{H}$ & -10.80708331 & 1.01681333 & 1.45888975 \\
\hline C & -5.26286166 & -5.91758228 & -0.98239411 \\
\hline $\mathrm{H}$ & -5.29479342 & -5.73869042 & 0.07206218 \\
\hline $\mathrm{H}$ & -5.98828054 & -6.66068039 & -1.24021702 \\
\hline C & -3.85881581 & -6.41220289 & -1.37686302 \\
\hline $\mathrm{H}$ & -3.27694946 & -5.58755842 & -1.73224034 \\
\hline $\mathrm{H}$ & -3.38019956 & -6.84483819 & -0.52325118 \\
\hline $\mathrm{H}$ & -3.94376050 & -7.14787692 & -2.14917650 \\
\hline C & -9.40194620 & -2.54254069 & 0.87120957 \\
\hline $\mathrm{H}$ & -9.43692048 & -2.16775153 & 1.87281322 \\
\hline $\mathrm{H}$ & -10.04607872 & -1.95731622 & 0.24871007 \\
\hline $\mathrm{H}$ & -9.72564591 & -3.56234630 & 0.86048441 \\
\hline C & -8.33764311 & -4.69427310 & -0.82744729 \\
\hline $\mathrm{H}$ & -8.04770023 & -5.05344552 & -1.79275983 \\
\hline $\mathrm{H}$ & -8.24108707 & -5.48215871 & -0.10994245 \\
\hline $\mathrm{H}$ & -9.35504444 & -4.36455997 & -0.86036808 \\
\hline C & 4.47574845 & -6.46266820 & 0.79425280 \\
\hline $\mathrm{H}$ & 3.54506452 & -6.77428936 & 1.22042140 \\
\hline $\mathrm{H}$ & 4.34902724 & -6.29415823 & -0.25476870 \\
\hline C & 5.53382898 & -7.55987877 & 1.01378542 \\
\hline 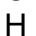 & 6.15254038 & -7.63780588 & 0.14428926 \\
\hline . & 6.13817053 & -7.30834162 & 1.86018979 \\
\hline 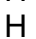 & 5.04593553 & -8.49583611 & 1.18940955 \\
\hline
\end{tabular}

$\begin{array}{cccc}\mathrm{C} & 8.62425033 & -0.21599956 & -1.04104877 \\ \mathrm{H} & 8.46938233 & 0.83080783 & -1.19951071 \\ \mathrm{H} & 8.58345496 & -0.42796156 & 0.00695307 \\ \mathrm{C} & 10.00387519 & -0.62018266 & -1.59320025 \\ \mathrm{H} & 10.65631959 & -0.86238830 & -0.78045630 \\ \mathrm{H} & 9.89699994 & -1.47263832 & -2.23100667 \\ \mathrm{H} & 10.41687616 & 0.19365028 & -2.15177535 \\ \mathrm{C} & 7.62822956 & -5.58782349 & 0.41531166 \\ \mathrm{H} & 7.43329938 & -5.86410894 & 1.43048089 \\ \mathrm{H} & 7.30653036 & -6.37330786 & -0.23616661 \\ \mathrm{H} & 8.67761701 & -5.42404555 & 0.28545494 \\ \mathrm{C} & 8.90085121 & -3.52329270 & -1.24964065 \\ \mathrm{H} & 8.97438155 & -3.05548807 & -2.20914684 \\ \mathrm{H} & 9.63751052 & -3.10898471 & -0.59345321 \\ \mathrm{H} & 9.06595587 & -4.57548713 & -1.35224337\end{array}$

Helicene 3 TS (no D3)

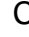

C

$\mathrm{H}$

$\mathrm{H}$

$\mathrm{H}$

$\mathrm{H}$

$\mathrm{H}$

$\mathrm{H}$

$\mathrm{C}$

C

C

C

C

C

C

C

C

C

C

$\mathrm{H}$

C

C
$-3.37829500-0.68070600-1.17610400$ $\begin{array}{llll}-2.20352100 & -1.44228300 & -1.22762900\end{array}$ $\begin{array}{lll}-2.18075100 & -2.81422500 & -0.98054200\end{array}$ $\begin{array}{lll}-3.40495300 & -3.50946200 & -0.77161300\end{array}$ $\begin{array}{lll}-4.58779500 & -2.76789500 & -0.78461500\end{array}$ $\begin{array}{lll}-0.92431600 & -3.43177700 & -0.54513600\end{array}$ $\begin{array}{lll}-1.05251900 & -4.44336300 & 0.46386800\end{array}$ $\begin{array}{lll}-2.21859700 & -5.36446100 & 0.37706400\end{array}$ $\begin{array}{lll}-3.34508500 & -4.94178400 & -0.40104000\end{array}$ $\begin{array}{lll}-4.60301100 & -1.37042600 & -0.95624100\end{array}$ $\begin{array}{lll}-3.35977300 & 0.77597900 & -1.13358800\end{array}$ $\begin{array}{lll}-2.17282700 & 1.50776000 & -1.20175700\end{array}$ $\begin{array}{lll}-2.10348400 & 2.87205400 & -0.92558500\end{array}$ $\begin{array}{lll}-3.28284700 & 3.59217300 & -0.60919000\end{array}$ $\begin{array}{lll}-4.48169000 & 2.87226600 & -0.56052800\end{array}$ $\begin{array}{llll}-0.80316000 & 3.49194300 & -0.69512000\end{array}$ $\begin{array}{lll}-0.72166700 & 4.59158200 & 0.22389900\end{array}$ $\begin{array}{lll}-1.93117500 & 5.46415900 & 0.36617300\end{array}$ $\begin{array}{lll}-3.15639000 & 5.02268700 & -0.25062200\end{array}$ $\begin{array}{llll}0.34786700 & 2.88226600 & -1.17540100\end{array}$ $\begin{array}{lll}1.61756700 & 3.13907700 & -0.65131200\end{array}$ $\begin{array}{llll}0.52985100 & 4.74702000 & 0.84448700\end{array}$ $\begin{array}{lll}-4.55041000 & 1.48979700 & -0.81761700\end{array}$ $\begin{array}{lll}1.68366400 & 4.03155100 & 0.45376900\end{array}$ $\begin{array}{lll}-5.81985700 & -0.60733700 & -0.79543500\end{array}$ $\begin{array}{lll}-5.78983400 & 0.76006500 & -0.68993700\end{array}$ $\begin{array}{lll}-1.25262000 & -0.93185700 & -1.29173400\end{array}$ $\begin{array}{lll}-5.52716300 & -3.24257000 & -0.54341100\end{array}$ $\begin{array}{llll}-1.24629000 & 0.97399700 & -1.35181900\end{array}$ $\begin{array}{llll}-5.39575900 & 3.35497900 & -0.25208400\end{array}$ $\begin{array}{lll}0.24016500 & 2.12164200 & -1.93745000\end{array}$ $\begin{array}{llll}0.66266400 & 5.39569600 & 1.69073000\end{array}$ $\begin{array}{lll}-4.20281600 & 5.94568400 & -0.52389600\end{array}$ $\begin{array}{lll}-1.90821100 & 6.77392100 & 0.93434100\end{array}$ $\begin{array}{lll}-3.03168900 & 7.61535300 & 0.80690900\end{array}$ $\begin{array}{lll}-4.11842600 & 7.25118200 & -0.01021200\end{array}$ $\begin{array}{lll}-4.33738000 & -5.87126900 & -0.80256800\end{array}$ $\begin{array}{lll}-2.18761700 & -6.69770700 & 0.87703300\end{array}$ $\begin{array}{lll}-3.24568100 & -7.57578200 & 0.56780600\end{array}$ $\begin{array}{lll}-4.27009700 & -7.19256700 & -0.32047900\end{array}$ $\begin{array}{lll}2.80810100 & 2.47373500 & -1.13442100\end{array}$ $\begin{array}{llll}2.94465600 & 4.17657000 & 1.14160700\end{array}$ $\begin{array}{lll}4.04453300 & 3.44222500 & 0.76448500\end{array}$ $\begin{array}{lll}4.02417600 & 2.59721500 & -0.40609400\end{array}$ $\begin{array}{lll}2.81791500 & 1.71093200 & -2.32219600\end{array}$ $\begin{array}{lll}1.92080300 & 1.63799800 & -2.92746100\end{array}$ $\begin{array}{llll}5.19644800 & 1.95829900 & -0.87133000\end{array}$ $\begin{array}{llll}5.18424800 & 1.19883100 & -2.04035400\end{array}$ $\begin{array}{lll}3.96678000 & 1.10611800 & -2.77003300\end{array}$ 


\begin{tabular}{|c|c|c|c|}
\hline $\mathrm{H}$ & 3.96635500 & 0.57459600 & -3.71630000 \\
\hline C & 0.32541000 & -2.87416800 & -0.81206800 \\
\hline C & 1.41053700 & -3.03699300 & 0.06678300 \\
\hline C & -0.04869800 & -4.44863100 & 1.43516900 \\
\hline C & 1.13669200 & -3.69748200 & 1.29969000 \\
\hline $\mathrm{H}$ & 0.43106900 & -2.19382900 & -1.65179200 \\
\hline $\mathrm{H}$ & -0.16953600 & -4.99267500 & 2.35798300 \\
\hline C & 2.06464000 & -3.55984400 & 2.39271000 \\
\hline C & 3.20365800 & -2.81087200 & 2.25756500 \\
\hline C & 6.36750800 & 0.81979100 & -2.75311300 \\
\hline C & 7.27328900 & 0.55641200 & -3.51738200 \\
\hline 0 & 8.39098600 & 0.18888700 & -4.38661800 \\
\hline & 8.23824200 & 0.61662200 & -5.38868300 \\
\hline $\mathrm{H}$ & 9.31829800 & 0.64165900 & -4.00613200 \\
\hline $\mathrm{H}$ & 6.12156000 & 2.10233400 & -0.32752000 \\
\hline C & 2.72197500 & -2.44881500 & -0.14625100 \\
\hline C & 3.57026600 & -2.24749200 & 0.97916500 \\
\hline C & 3.24115600 & -2.12629300 & -1.41946600 \\
\hline t & 2.65203200 & -2.36182400 & -2.30157600 \\
\hline C & 4.80166900 & -1.59347700 & 0.80634300 \\
\hline C & 5.30015900 & -1.24953500 & -0.46049100 \\
\hline C & 4.51461400 & -1.60613400 & -1.58383800 \\
\hline $\mathrm{H}$ & 4.92138000 & -1.47788500 & -2.57954500 \\
\hline C & 6.68299200 & -0.88423100 & -0.47302400 \\
\hline C & 7.85806300 & -0.63382600 & -0.30284300 \\
\hline C & 9.27956300 & -0.31229800 & -0.19514600 \\
\hline $\mathrm{H}$ & 9.56566700 & 0.33872700 & -1.03485800 \\
\hline $\mathrm{H}$ & 9.45779300 & 0.27572700 & 0.71711100 \\
\hline $\mathrm{H}$ & 5.42214000 & -1.42536100 & 1.67869900 \\
\hline $\mathrm{O}$ & 2.98854300 & 5.03194900 & 2.22267300 \\
\hline $\mathrm{O}$ & 5.23456600 & 3.53946000 & 1.45737200 \\
\hline C & 5.22913000 & 2.87157400 & 2.72630300 \\
\hline $\mathrm{H}$ & 6.23208600 & 2.99954500 & 3.14061900 \\
\hline $\mathrm{H}$ & 4.48945400 & 3.31554700 & 3.40076400 \\
\hline 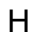 & 5.01922100 & 1.80247000 & 2.60015800 \\
\hline C & 3.88179500 & 6.14578800 & 2.07616700 \\
\hline 1 & 3.77890300 & 6.73696400 & 2.98883200 \\
\hline & 4.91578600 & 5.81056900 & 1.96227800 \\
\hline $\mathrm{H}$ & 3.59563200 & 6.75773100 & 1.21156700 \\
\hline 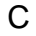 & -0.71413000 & 7.36007600 & 1.67098000 \\
\hline $\mathrm{H}$ & 0.21717800 & 7.08627200 & 1.17229400 \\
\hline $\mathrm{H}$ & -0.74688700 & 8.45076900 & 1.60423100 \\
\hline C & -5.36825400 & 5.59445800 & -1.44441400 \\
\hline $\mathrm{H}$ & -5.14978300 & 4.67246800 & -1.98553300 \\
\hline 1 & -5.44090500 & 6.36771900 & -2.22153000 \\
\hline C & -5.40453100 & -5.51639300 & -1.83344700 \\
\hline $\mathrm{H}$ & -5.14474200 & -4.57979600 & -2.33173000 \\
\hline $\mathrm{H}$ & -5.38021800 & -6.27743000 & -2.62536900 \\
\hline C & -1.01774500 & -7.25903700 & 1.67220600 \\
\hline $\mathrm{H}$ & -0.08950000 & -6.77422600 & 1.36185300 \\
\hline $\mathrm{H}$ & -0.88290200 & -8.31603700 & 1.41856300 \\
\hline O & -6.93861100 & 1.49385200 & -0.47573600 \\
\hline $\mathrm{O}$ & -6.99986200 & -1.31100600 & -0.65715000 \\
\hline C & -7.54885600 & 1.30918500 & 0.80940900 \\
\hline $\mathrm{H}$ & -8.40640100 & 1.98550100 & 0.83511300 \\
\hline & -7.88247600 & 0.27622300 & 0.94411500 \\
\hline $\mathrm{H}$ & -6.84870600 & 1.57636900 & 1.61050200 \\
\hline & -7.91236000 & -1.15780000 & -1.75125200 \\
\hline $\mathrm{H}$ & -8.77001100 & -1.79481400 & -1.52301600 \\
\hline & -8.23678100 & -0.11725600 & -1.85185100 \\
\hline & -7.45133700 & -1.49028400 & -2.68983100 \\
\hline & 1.76564600 & -4.19605400 & 3.58163200 \\
\hline 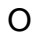 & 4.05189900 & -2.59940800 & 3.32472000 \\
\hline & 4.83682400 & -3.73970200 & 3.69790500 \\
\hline & 5.47447300 & -3.41191000 & 4.52252500 \\
\hline & 4.19848200 & -4.56556700 & 4.02711100 \\
\hline & 5.46457100 & -4.06875400 & 2.86040700 \\
\hline
\end{tabular}

C
$1.30563100 \quad-3.32412300 \quad 4.62358200$ $\begin{array}{lll}1.08929200 & -3.96452100 & 5.48190100\end{array}$ $2.07561400 \quad-2.59310800 \quad 4.88890700$ $\begin{array}{lll}0.39057400 & -2.80261300 & 4.31688600\end{array}$ $\begin{array}{lll}-5.16185600 & 8.30559300 & -0.33352100\end{array}$ $\begin{array}{lll}-5.73351700 & 8.06484100 & -1.22995100\end{array}$ $\begin{array}{lll}-5.88511400 & 8.43778800 & 0.48291300\end{array}$ $\begin{array}{lll}-4.69566700 & 9.28092400 & -0.50467100\end{array}$ $\begin{array}{lll}-3.07202900 & 8.96941400 & 1.49788900\end{array}$ $\begin{array}{lll}-2.59989200 & 9.76199600 & 0.89917800\end{array}$ $\begin{array}{lll}-4.10048000 & 9.28430100 & 1.68764900\end{array}$ $\begin{array}{lll}-2.56801200 & 8.95295600 & 2.46604400\end{array}$ $-6.75418100 \quad 5.46695700-0.77254400$ $\begin{array}{lll}-6.68504100 & 4.82312000 & 0.11310100\end{array}$ $\begin{array}{llll}-7.07497600 & 6.44503300 & -0.39644400\end{array}$ $\begin{array}{lll}-7.81435900 & 4.90785300 & -1.72706200\end{array}$ $\begin{array}{lll}-7.88330900 & 5.51006200 & -2.64185700\end{array}$ $\begin{array}{lll}-7.57617700 & 3.87775000 & -2.01501100\end{array}$ $\begin{array}{llll}-8.80569600 & 4.90186100 & -1.25876200\end{array}$ $\begin{array}{lll}-0.66401200 & 6.97224300 & 3.16535700\end{array}$ $\begin{array}{lll}-0.65268700 & 5.87993600 & 3.26601800\end{array}$ $-6.85521000 \quad-5.41892000-1.31465100$ $\begin{array}{lll}-7.12529300 & -6.33754400 & -0.78140200\end{array}$ $\begin{array}{lll}-6.93756100 & -4.61404100 & -0.57439300\end{array}$ $\begin{array}{lll}-7.85758100 & -5.17498900 & -2.44758100\end{array}$ $\begin{array}{lll}-7.83655900 & -5.99230300 & -3.17944100\end{array}$ $-8.88183700 \quad-5.09797200-2.06483500$ $\begin{array}{lll}-7.63078000 & -4.24667800 & -2.98622200\end{array}$ $\begin{array}{lll}-1.16638000 & -7.14746400 & 3.20665000\end{array}$ $\begin{array}{lll}-2.02189200 & -7.75231100 & 3.53382700\end{array}$ $\begin{array}{lll}-1.42379600 & -6.11673100 & 3.48169600\end{array}$ $\begin{array}{lll}0.09643400 & -7.58411000 & 3.95667800\end{array}$ $\begin{array}{llll}0.93804000 & -6.91863100 & 3.73208100\end{array}$ $\begin{array}{lll}-0.06168100 & -7.56701800 & 5.04144600\end{array}$ $\begin{array}{lll}0.38934000 & -8.60482500 & 3.68006300\end{array}$ $\begin{array}{lll}-5.26172900 & -8.25320300 & -0.76448100\end{array}$ $\begin{array}{lll}-6.00954900 & -8.46509700 & 0.01222600\end{array}$ $\begin{array}{lll}-5.80771400 & -7.97053200 & -1.66418500\end{array}$ $-4.75678900 \quad-9.20035900-0.98060300$ $\begin{array}{lll}-3.27204600 & -8.99169400 & 1.11875000\end{array}$ $\begin{array}{lll}-2.71565500 & -9.09225300 & 2.04989400\end{array}$ $\begin{array}{lll}-4.29630900 & -9.31351000 & 1.32683900\end{array}$ $\begin{array}{lll}-2.85049000 & -9.71719700 & 0.40759300\end{array}$ $\begin{array}{lll}-1.59618000 & 7.29994100 & 3.64443900\end{array}$ $\begin{array}{lll}0.53493400 & 7.57409800 & 3.90367200\end{array}$ $\begin{array}{lll}0.50725000 & 7.32827800 & 4.97138400\end{array}$ $\begin{array}{lll}1.47774400 & 7.18903500 & 3.49963600\end{array}$ $\begin{array}{lll}0.55123400 & 8.66759500 & 3.81255600\end{array}$ $\begin{array}{lll}8.58794100 & -1.33819900 & -4.51469200\end{array}$ $8.72681300-1.76224800-3.51291500$ $\begin{array}{lll}7.66639700 & -1.78159900 & -4.91165600\end{array}$ $9.77352600-1.69839000-5.41347200$ $10.71128800-1.28586200-5.02088400$ $9.89461000-2.78451800-5.49103900$ $9.63813100-1.30504100-6.42850400$ $10.19526000-1.55608300-0.17768900$ $9.90035500 \quad-2.20262000 \quad 0.65793500$ $10.02291500-2.13671000-1.09252600$ $11.67612500-1.18649900-0.05982100$ $11.87426600 \quad-0.62765900 \quad 0.86315300$ $12.30784400-2.08160400-0.04896100$ $11.99905500-0.56089700 \quad-0.90112100$ 


\section{References}

(1) Nitschke, J. R.; Zürcher, S.; Tilley, T. D. New Zirconocene-Coupling Route to Large, Functionalized Macrocycles. J. Am. Chem. Soc. 2000, 122 (42), 10345-10352. http://dx.doi.org/10.1021/ja0020310.

(2) Dutton, J. L.; Farrar, G. J.; Sgro, M. J.; Battista, T. L.; Ragogna, P. J. Lewis Base Sequestered Chalcogen Dihalides: Synthetic Sources of $\mathrm{ChX} 2(\mathrm{Ch}=\mathrm{Se}, \mathrm{Te} ; \mathrm{X}=\mathrm{Cl}$, Br). Chem. - Eur. J. 2009, 15 (39), 10263-10271. https://doi.org/10.1002/chem.200901000.

(3) Herde, J. L.; Lambert, J. C.; Senoff, C. V.; Cushing, M. A. Cyclooctene and 1,5Cyclooctadiene Complexes of Iridium(I). In Inorganic Syntheses; Parshall, G. W., Ed.; John Wiley \& Sons, Inc., 1974; pp 18-20. https://doi.org/10.1002/9780470132463.ch5.

(4) Brandsma, L. Best Synthetic Methods: Acetylenes, Allenes and Cumulenes, 1st ed.; Elsevier Academic Press: Oxford, UK, 2003.

(5) von Kugelgen, S.; Bellone, D. E.; Cloke, R. R.; Perkins, W. S.; Fischer, F. R. Initiator Control of Conjugated Polymer Topology in Ring-Opening Alkyne Metathesis Polymerization. J. Am. Chem. Soc. 2016, 138 (19), 6234-6239. https://doi.org/10.1021/jacs.6b02422.

(6) Makino, T.; Yamamoto, Y.; Itoh, K. Synthesis and Structure of Novel Iridium(I) Complexes Containing $\eta^{4}-1,6$-Diene and Diphosphine Ligands: Remarkable Effect of Ligand Bite Angle upon Ligand Dissociation. Organometallics 2004, 23 (8), 17301737. https://doi.org/10.1021/om034282y.

(7) Kiel, G. R.; Patel, S. C.; Smith, P. W.; Levine, D. S.; Tilley, T. D. Expanded Helicenes: A General Synthetic Strategy and Remarkable Supramolecular and Solid-State Behavior. J. Am. Chem. Soc. 2017, 139 (51), 18456-18459. https://doi.org/10.1021/jacs.7b10902.

(8) Kiel, G. R.; Bergman, H. M.; Tilley, T. D. Site-Selective [2+2+ n] Cycloadditions for Rapid, Scalable Access to Alkynylated Polycyclic Aromatic Hydrocarbons. Chem. Sci. 2020, 11 (11), 3028-3035. https://doi.org/10.1039/C9SC06102A.

(9) Krasovskiy, A.; Knochel, P. A LiCl-Mediated Br/Mg Exchange Reaction for the Preparation of Functionalized Aryl- and Heteroarylmagnesium Compounds from Organic Bromides. Angew. Chem. Int. Ed. 2004, 43 (25), 3333-3336. https://doi.org/10.1002/anie.200454084.

(10) O'Brien, C. J.; Kantchev, E. A. B.; Valente, C.; Hadei, N.; Chass, G. A.; Lough, A.; Hopkinson, A. C.; Organ, M. G. Easily Prepared Air- and Moisture-Stable Pd-NHC ( $\mathrm{NHC}=\mathrm{N}$-Heterocyclic Carbene) Complexes: A Reliable, User-Friendly, Highly Active Palladium Precatalyst for the Suzuki-Miyaura Reaction. Chem. - Eur. J. 2006, 12 (18), 4743-4748. https://doi.org/10.1002/chem.200600251.

(11) Hoye, T. R.; Eklov, B. M.; Voloshin, M. No-D NMR Spectroscopy as a Convenient Method for Titering Organolithium (RLi), RMgX, and LDA Solutions. Org. Lett. 2004, 6 (15), 2567-2570. https://doi.org/10.1021/ol049145r.

(12) Vaska, L.; Catone, D. L. A New Series of Four-, Five-, and Six-Coordinated Iridium Complexes. J. Am. Chem. Soc. 1966, 88 (22), 5324-5325. https://doi.org/10.1021/ja00974a054. 
(13) Lilga, M. A.; Ibers, J. A. Oxidation of the Carbonylbis(1,2Bis(Diphenylphosphino)Ethane)Iridium(I) Cation. Preparation and Characterization of a Series of Iridium(III) Carbonyl Dications. Inorg. Chem. 1984, 23 (22), 35383543. https://doi.org/10.1021/ic00190a021.

(14) Rickhaus, M.; Jundt, L.; Mayor, M. Determining Inversion Barriers in Atropisomers A Tutorial for Organic Chemists. Chim. Int. J. Chem. 2016, 70 (3), 192-202. https://doi.org/info:doi/10.2533/chimia.2016.192.

(15) Klan, P.; Wirz, J. Photochemistry of Organic Compounds: From Concepts to Practice, Pg. 194; John Wiley \& Sons, Ltd., 2009.

(16) Sheldrick, G. M. A Short History of SHELX. Acta Crystallogr. A 2008, 64 (1), 112122. https://doi.org/10.1107/S0108767307043930.

(17) Sheldrick, G. M. SHELXT - Integrated Space-Group and Crystal-Structure Determination. Acta Crystallogr. Sect. Found. Adv. 2015, 71 (1), 3-8. https://doi.org/10.1107/S2053273314026370.

(18) Palatinus, L.; Chapuis, G. SUPERFLIP - a Computer Program for the Solution of Crystal Structures by Charge Flipping in Arbitrary Dimensions. J. Appl. Crystallogr. 2007, 40 (4), 786-790. https://doi.org/10.1107/S0021889807029238.

(19) Sheldrick, G. M. Crystal Structure Refinement with SHELXL. Acta Crystallogr. Sect. C Struct. Chem. 2015, 71 (1), 3-8. https://doi.org/10.1107/S2053229614024218.

(20) Macrae, C. F.; Edgington, P. R.; McCabe, P.; Pidcock, E.; Shields, G. P.; Taylor, R.; Towler, M.; Streek, J. van de. Mercury: Visualization and Analysis of Crystal Structures. J. Appl. Crystallogr. 2006, 39 (3), 453-457. https://doi.org/10.1107/S002188980600731X.

(21) Spek, A. L. PLATON SQUEEZE: A Tool for the Calculation of the Disordered Solvent Contribution to the Calculated Structure Factors. Acta Crystallogr. Sect. C Struct. Chem. 2015, 71 (1), 9-18. https://doi.org/10.1107/S2053229614024929.

(22) Frisch, M. J.; Trucks, G. W.; Schlegel, H. B.; Scuseria, G. E.; Robb, M. A.; Cheeseman, J. R.; Scalmani, G.; Barone, V.; Mennucci, B.; Petersson, G. A.; Nakatsuji, H.; Caricato, M.; Li, X.; Hratchian, H. P.; Izmaylov, A. F.; Bloino, J.; Zheng, G.; Sonnenberg, J. L.; Hada, M.; Ehara, M.; Toyota, K.; Fukuda, R.; Hasegawa, J.; Ishida, M.; Nakajima, T.; Honda, Y.; Kitao, O.; Nakai, H.; Vreven, T.; Montgomery, J. A.; Peralta, J. E.; Ogliaro, F.; Bearpark, M.; Heyd, J. J.; Brothers, E.; Kudin, K. N.; Staroverov, V. N.; Kobayashi, R.; Normand, J.; Raghavachari, K.; Rendell, A.; Burant, J. C.; lyengar, S. S.; Tomasi, J.; Cossi, M.; Rega, N.; Millam, J. M.; Klene, M.; Knox, J. E.; Cross, J. B.; Bakken, V.; Adamo, C.; Jaramillo, J.; Gomperts, R.; Stratmann, R. E.; Yazyev, O.; Austin, A. J.; Cammi, R.; Pomelli, C.; Ochterski, J. W.; Martin, R. L.; Morokuma, K.; Zakrzewski, V. G.; Voth, G. A.; Salvador, P.; Dannenberg, J. J.; Dapprich, S.; Daniels, A. D.; Farkas; Foresman, J. B.; Ortiz, J. V.; Cioslowski, J.; Fox, D. J. Gaussian 09; Gaussian Inc.: Wallingford, CT, 2009.

(23) Becke, A. D. Density-functional Thermochemistry. III. The Role of Exact Exchange. J. Chem. Phys. 1993, 98 (7), 5648-5652. https://doi.org/10.1063/1.464913.

(24) Lee, C.; Yang, W.; Parr, R. G. Development of the Colle-Salvetti Correlation-Energy Formula into a Functional of the Electron Density. Phys. Rev. B 1988, 37 (2), 785789. https://doi.org/10.1103/PhysRevB.37.785.

(25) Becke, A. D. A New Mixing of Hartree-Fock and Local Density-functional Theories. J. Chem. Phys. 1993, 98 (2), 1372-1377. https://doi.org/10.1063/1.464304. 
(26) Stephens, P. J.; Devlin, F. J.; Chabalowski, C. F.; Frisch, M. J. Ab Initio Calculation of Vibrational Absorption and Circular Dichroism Spectra Using Density Functional Force Fields. J. Phys. Chem. 1994, 98 (45), 11623-11627. https://doi.org/10.1021/j100096a001.

(27) Ditchfield, R.; Hehre, W. J.; Pople, J. A. Self-Consistent Molecular-Orbital Methods. IX. An Extended Gaussian-Type Basis for Molecular-Orbital Studies of Organic Molecules. J. Chem. Phys. 1971, 54 (2), 724-728. https://doi.org/10.1063/1.1674902.

(28) Hehre, W. J.; Ditchfield, R.; Pople, J. A. Self-Consistent Molecular Orbital Methods. XII. Further Extensions of Gaussian-Type Basis Sets for Use in Molecular Orbital Studies of Organic Molecules. J. Chem. Phys. 1972, 56 (5), 2257-2261. https://doi.org/10.1063/1.1677527.

(29) Legault, C. Y. CYLview, 1.0b; Universitéde Sherbrooke, 2009 (http://www.cylview.org). 